2014

\title{
Investigating the Role of Mechanical Properties in Biological Systems
}

Nicole Shamitko-Klingensmith

Follow this and additional works at: https://researchrepository.wvu.edu/etd

\section{Recommended Citation}

Shamitko-Klingensmith, Nicole, "Investigating the Role of Mechanical Properties in Biological Systems" (2014). Graduate Theses, Dissertations, and Problem Reports. 6613.

https://researchrepository.wvu.edu/etd/6613 
Investigating the Role of Mechanical Properties in Biological Systems

Nicole Shamitko-Klingensmith

\author{
Dissertation submitted \\ to the Eberly College of Arts and Sciences \\ at West Virginia University
}

in partial fulfillment of the requirements for the degree of

Doctor of Philosophy in

Physical Chemistry

\author{
Justin Legleiter, Ph.D., Chair \\ Jonathan Boyd, Ph.D. \\ Terry Gullion, Ph.D. \\ Michelle Richards-Babb, Ph.D. \\ Yong Yang, Ph.D. \\ C. Eugene Bennett Department of Chemistry \\ Morgantown, West Virginia \\ 2014
}

Keywords: atomic force microscopy, scanning probe acceleration microscopy, mechanical properties, biological membranes

Copyright 2014 Nicole Shamitko-Klingensmith 


\section{ABSTRACT \\ Investigating the Role of Mechanical Properties in Biological Systems \\ Nicole Shamitko-Klingensmith}

There is considerable interest in measuring, with nanoscale spatial resolution, the physical and material properties of biological membranes and whole cells because of their role in the physiology of living systems. The atomic force microscope (AFM) has proven to be particularly well-suited for biological studies because samples can be maintained in near physiological conditions to preserve sample integrity (such as in a buffer solution at a physiologically relevant $\mathrm{pH}$ or temperature), and the imaging process is relatively nondestructive. In this work, the mechanical properties of supported lipid bilayers and neurons were examined using AFM-based techniques. Specifically, tapping mode AFM-based techniques were used to investigate the influence of lipid composition and temperature fluctuations on the physicomechanical properties of supported lipid bilayers. Topographic images were produced by tapping mode AFM, while additional compositional contrast and mechanical information was gained from phase contrast imaging, higher harmonic imaging, and scanning probe acceleration microscopy (SPAM). Importantly, all of this data was acquired simultaneously during the tapping mode AFM imaging process and the same imaging parameters were used for each experiment so that fair mechanical comparisons could be made across experiments. Lastly, force-distance curves and force volume imaging were used to better understand the effect of microtubule disruption or stabilization on the toxic ability of amyloid- $\beta_{1-42}$ aggregates, which are implicated in the development of Alzheimer's disease. Overall, the work presented in this dissertation improved techniques for studies in mechanobiology and examined how biologically relevant factors affect the mechanical properties of lipid membranes or whole cells. 


\section{Acknowledgments}

I would like to express my appreciation and gratitude to those who have supported me throughout my graduate career.

First, I am extremely grateful to have had such a wonderful advisor, Dr. Justin Legleiter. Thank you for accepting me into your group, and for having the patience to work with me over the past few years. You have taught me so much, and you made graduate school a fun experience. I truly admire your dedication to your work; you set an excellent example for your students.

I would also like to acknowledge Dr. Jonathan Boyd, who became my co-advisor for the IGERT (Integrative Graduate Education and Research Traineeship) fellowship program. Thank you very much for your guidance with experiments and the writing process. I am very appreciative to have had the opportunity to work with you.

I would also like to thank my current and former graduate committee members, Drs. Terry Gullion, Michelle Richards-Babb, Yong Yang, Ken Showalter, and Pete Gannett, for their support and advice throughout my graduate career.

To my former labmates, Drs. Elizabeth Yates and Kathleen Burke, thank you for guiding me to the group, and for helping to train me during my early days in the lab. To my current labmates, Maxmore Chaibva, Jim Arndt, Xiang Gao, and Albert Pilkington, thank you all very much for your support, encouragement, and friendship.

I would also like to acknowledge several other people who were involved in my research projects. Thanks to Kelley Molchanoff (Wambaugh) and George Magnone who helped with the SPAM procedure and the simulations that are presented in Chapters 2-3. Dr. Holly Currie, thank you for taking the time to train me in cell culture. Thanks to Julie Vrana, who provided me with cells for preliminary work to Chapter 5. I am also very grateful to Drs. Huiyuan Li and Kathy Brundage for fluorescence microscopy and flow cytometry assistance, respectively.

Finally, I would like to thank my family for their love and encouragement throughout this process, especially my parents, Thomas and Dawn Shamitko, and my husband, Zack Klingensmith. 


\section{Table of contents}

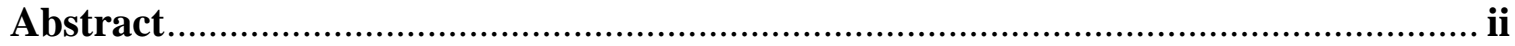

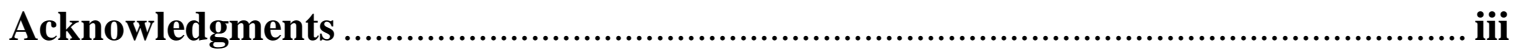

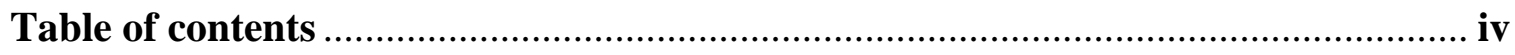

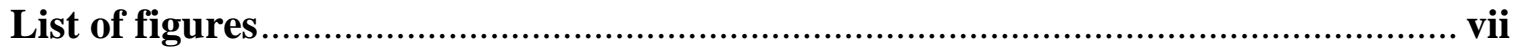

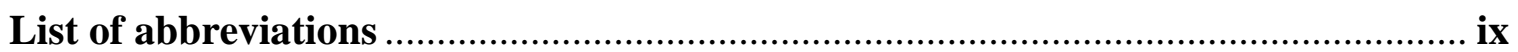

1. Probing the importance of mechanical properties in biological systems using the

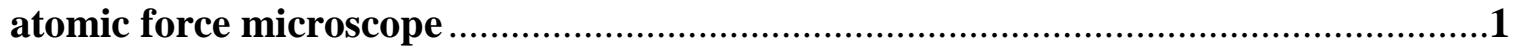

1.1 Alterations in biological mechanics contribute to disease .......................................1

1.2 Model systems to study biological mechanics .......................................................

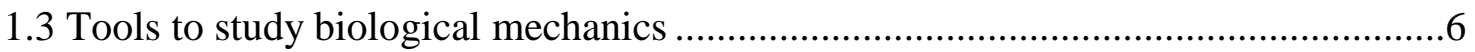

1.4 Development and operational principles of AFM ..............................................

1.4.1 History and fundamentals of the AFM ........................................................

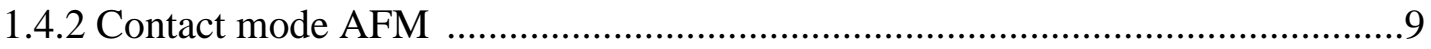

1.4.3 Tapping mode AFM ..............................................................................10

1.4.4 Phase contrast imaging ................................................................................11

1.4.5 Higher harmonics imaging ………...........................................................12

1.4.6 Force-distance curves and force volume imaging ..........................................12

1.4.7 Scanning probe acceleration microscopy ………..............................................14

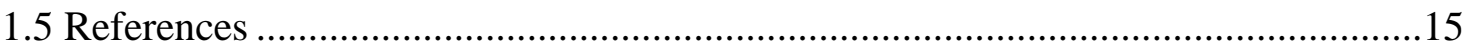

2. Scanning probe acceleration microscopy: theory and experiments ......................24

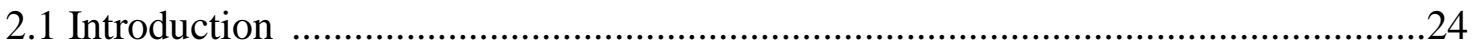

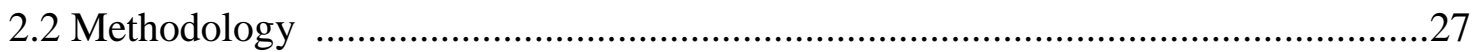

2.2.1 Numerical model of the entire imaging process of TMAFM in solution .........27

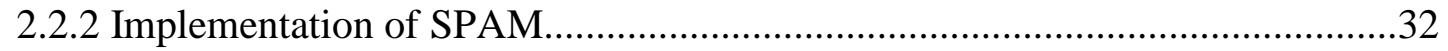

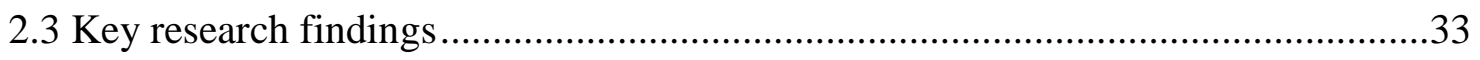

2.3.1 Features of the tip/sample force are independent of surface topography ..........33

2.3.2 Features of the tip/sample force provide insight into surface mechanical

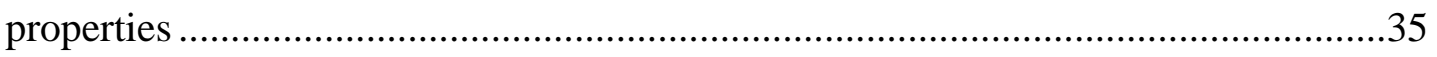

2.3.3 The impact of imaging parameters on the tip/sample force ...............................39

2.3.4 Experimental applications of SPAM ............................................................42

Table of Contents iv 


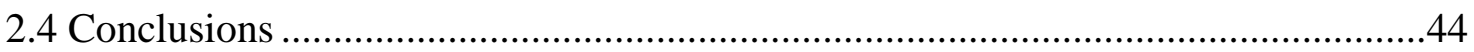

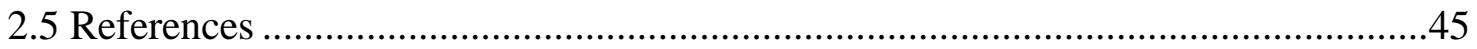

3. Mapping the mechanical properties of cholesterol-containing supported lipid

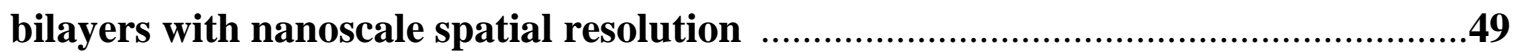

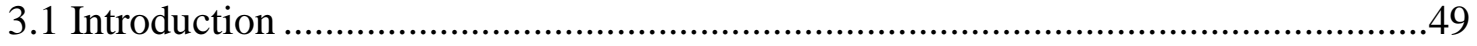

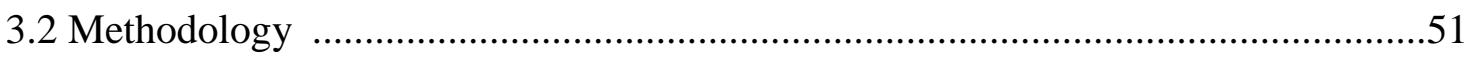

3.2.1 Sample preparation ................................................................................51

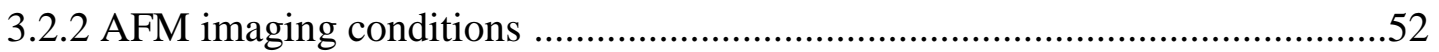

3.2.3 Scanning probe acceleration microscopy analysis ............................................52

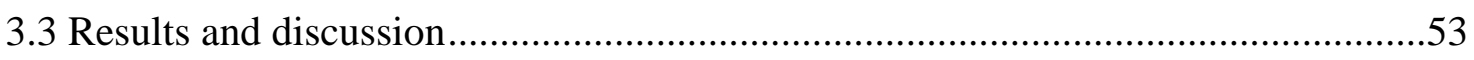

3.3.1 The role of set-point on time-resolved tip/sample force ……............................53

3.3.2 Physical and mechanical properties of TBLE supported bilayers change as a

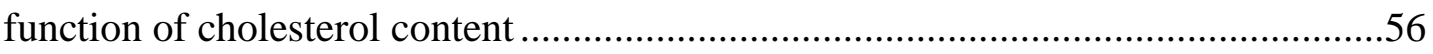

3.3.3 Material properties of lipid rafts are distinguishable from the surrounding supported lipid bilayer .........................................................................................63

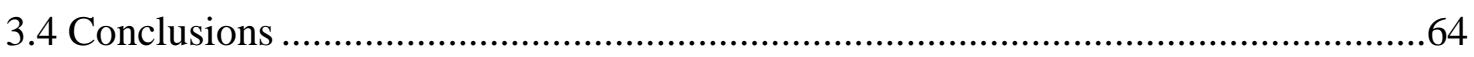

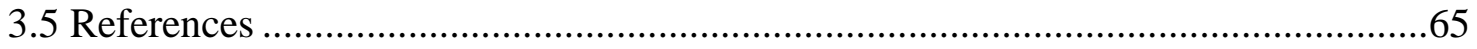

4. Investigation of temperature-induced mechanical changes in supported bilayers by variants of tapping mode atomic force microscopy ...........................................70

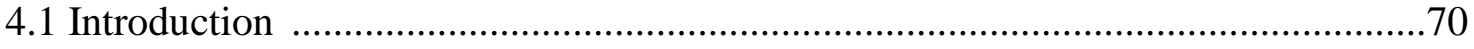

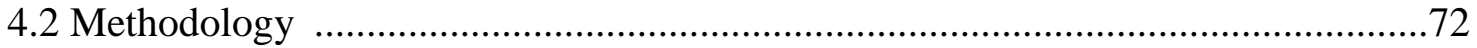

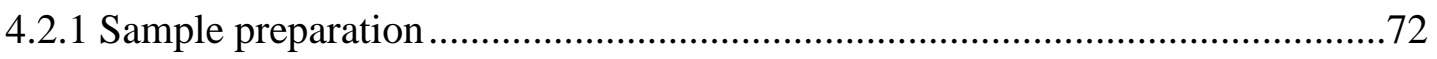

4.2.2 AFM imaging conditions...........................................................................73

4.2.3 Higher harmonic imaging and scanning probe acceleration microscopy ...........73

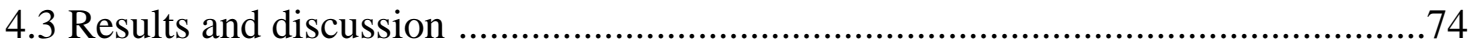

4.3.1 Influence of temperature on bilayer height and phase.......................................74

4.3.2 Observation of compositional contrast using higher harmonic imaging ...........81

4.3.3 Maximum tapping forces reflect temperature-induced mechanical changes in

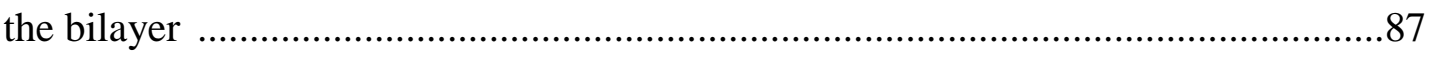

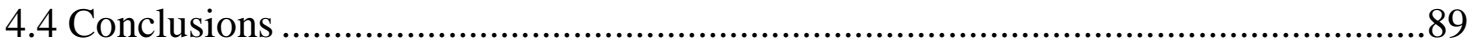

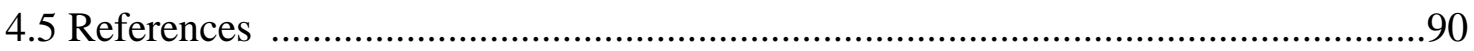

Table of Contents $v$ 


\section{Microtubule modification influences the cellular response to amyloid- $\beta_{1-42}$}

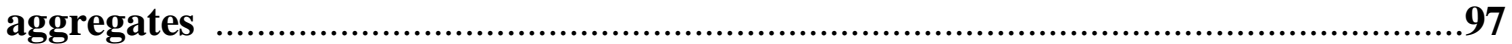

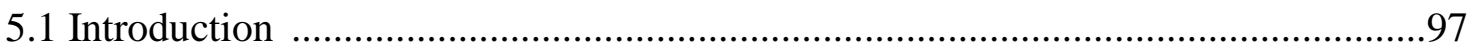

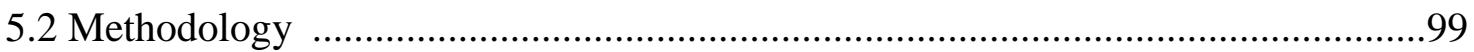

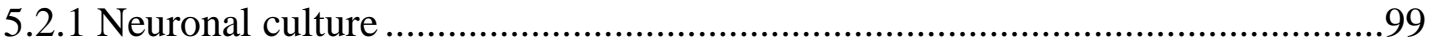

5.2.2 Microtubule treatments and $A \beta_{1-42}$ preparation .............................................100

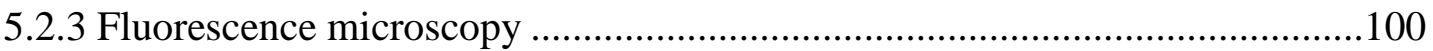

5.2.4 Cellular mechanical evaluation via AFM .....................................................101

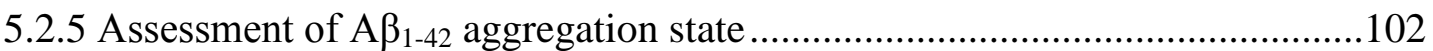

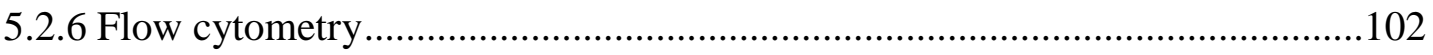

5.2.7 Plasma membrane degradation assay ……………........................................103

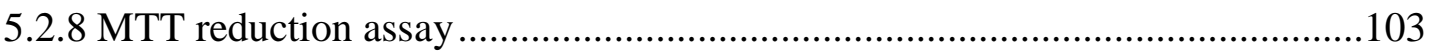

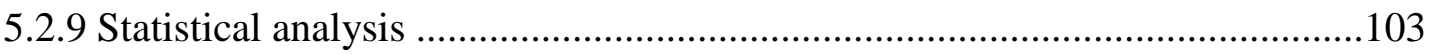

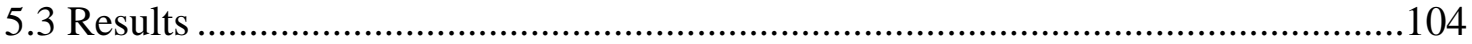

5.3.1 Microtubule modification alters the physical and mechanical properties of neurons

5.3.2 Microtubule disruption reduces the ability of $A \beta_{1-42}$ to bind to the cell membrane

5.3.3 Exposure to $A \beta_{1-42}$ alters neuronal rigidity

5.3.4 Microtubule disruption reduces the ability of neurons to cope with $\mathrm{A} \beta_{1-42}$ exposure

5.3.5 Microtubule stabilization delays the onset of $A \beta_{1-42}$-induced toxicity ............117

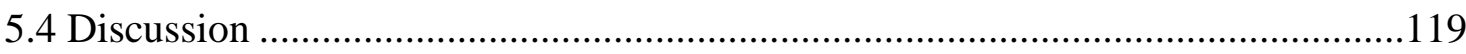

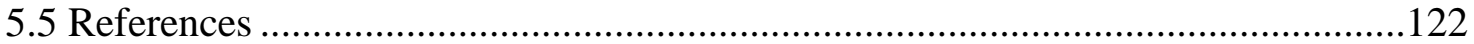




\section{List of figures}

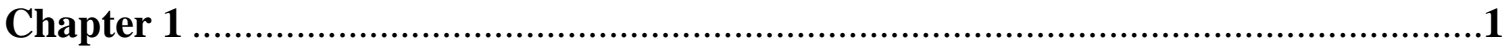

Figure 1. Cross-sectional view of membrane structure and components.......................3

Figure 2. Schematic illustration of the key components of an AFM ...........................8

Figure 3. Principle of phase contrast imaging ....................................................11

Figure 4. Illustration of a force-distance curve ....................................................13

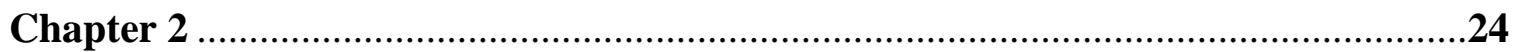

Figure 1. Principle of scanning probe acceleration microscopy ................................26

Figure 2. Numerical simulation of the entire TMAFM imaging process.....................27

Figure 3. Simulated cantilever position and deflection trajectories ..........................29

Figure 4. Comparing simulated and experimental deflection and force trajectories ....31

Figure 5. The effect of surface topography on tip/sample imaging forces....................34

Figure 6. The impact of surface Young's modulus on tip/sample imaging forces ........36

Figure 7. The impact of the Hamaker constant on tip/sample imaging forces ..............38

Figure 8. Average tapping force per oscillation cycle changes in response to cantilever

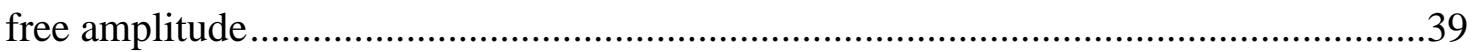

Figure 9. Effect of amplitude set-point on the tapping force per oscillation cycle ........40

Figure 10. The impact of cantilever spring constant on tip/sample imaging forces ......41

Figure 11. SPAM images of a supported lipid bilayer patch on mica ..........................42

Figure 12. SPAM images of polyglutamine peptide aggregates deposited on mica ......43

Figure 13. SPAM images of a blend of polystyrene and polyolefin elastomer ...........44

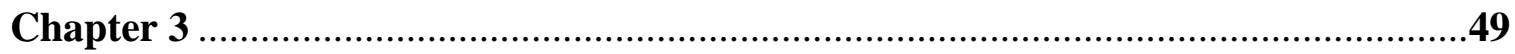

Figure 1. Schematic illustration of a total brain lipid extract (TBLE) bilayer ..............51

Figure 2. Effect of set-point ratio on the relative $F_{\min }$ and $F_{\max }$ on mica and supported

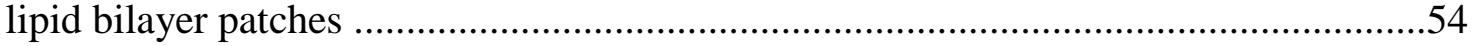

Figure 3. Effect of cholesterol content on the measured height of a TBLE bilayer.......57

Figure 4. Reconstructed $F_{\max }$ images of TBLE/cholesterol bilayer patches on mica.....59

Figure 5. Reconstructed $F_{\min }$ images of TBLE/cholesterol bilayer patches on mica .....60 
Figure 6. Average values of $F_{\max }$ and $F_{\min }$ on supported TBLE bilayer patches as a function of exogenous cholesterol content .61

Figure 7. Domains formed in TBLE/cholesterol systems at elevated temperatures ......64

\section{Chapter 4}

Figure 1. Surface topography of a TBLE bilayer patch supported on mica..................76

Figure 2. Cantilever phase response to imaging a TBLE bilayer patch on mica .78

Figure 3. Phase images of TBLE bilayer patches supported on mica from two additional experiments .79

Figure 4. Images and histograms corresponding to the $2^{\text {nd }}$ harmonic .82

Figure 5. Images and histograms corresponding to the $5^{\text {th }}$ harmonic. .83

Figure 6. Images and histograms corresponding to the $6^{\text {th }}$ harmonic. .84

Figure 7. Images and histograms corresponding to the $7^{\text {th }}$ harmonic. .85

Figure 8. Images and histograms corresponding to the $10^{\text {th }}$ and $15^{\text {th }}$ harmonics .86

Figure 9. Maximum tapping forces $\left(F_{\max }\right)$ associated with imaging a TBLE bilayer patch supported on mica.

Chapter 5 .97

Figure 1. Morphological changes of GT1-7 neurons treated with MT modifiers ........105

Figure 2. Measuring the E of neurons treated with MT modifiers............................106

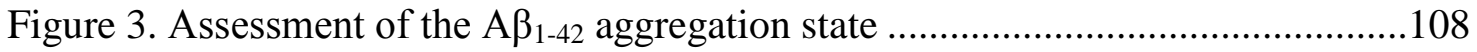

Figure 4. Aggregate-membrane binding as determined by flow cytometry ...............110

Figure 5. Fluorescence images of neurons with a MT modifier and FAM-A $\beta_{1-42} \ldots \ldots . .111$

Table 1. Percentage difference in $\mathrm{E}$ after exposure to $\mathrm{A} \beta_{1-42}$ aggregates ....................112

Figure 6. Measuring the E of neurons treated with a MT modifier and A $\beta \ldots \ldots \ldots \ldots . . . . .113$

Figure 7. Cellular response to $A \beta_{1-42}$ after colchicine treatment.............................115

Figure 8. Cellular response to $A \beta_{1-42}$ after nocodazole treatment .............................116

Figure 9. Cellular response to $\mathrm{A} \beta_{1-42}$ after paclitaxel treatment ..............................118 


\section{List of abbreviations}

$A$, cantilever tapping amplitude

$A_{\text {o, }}$ cantilever free tapping amplitude,

$a_{D M T}$, interatomic distance

$a_{0}$, drive oscillation amplitude

$\mathrm{A} \beta$, beta amyloid

$\mathrm{AD}$, Alzheimer's disease

AFM, atomic force microscopy

APP, amyloid precursor protein

$b$, damping coefficient

CMAFM, contact mode atomic force microscopy

$\delta$, Dirac's delta function

$D_{0}$, resting position of the cantilever base

DAPI, 4',6-diamidino-2-phenylindole

DLVO, Derjaguin-Landau-Verway-Overbeek

DMEM, Dulbecco's Modified Eagle Medium

DMSO, dimethyl sulfoxide

DMT, Derjaguin-Muller-Toporov

$E$, Young's modulus

ECM, extracellular matrix

EDTA, ethylenediaminetetraacetic acid

EGTA, ethylene glycol tetraacetic acid

$F_{\text {ext }}$, tip/sample tapping force

$F_{\max }$, maximum tapping force

$F_{\text {min }}$, minimum tapping force

$F_{\text {total }}$, total tapping force

FAM, fluorescein amidite

FBS, fetal bovine serum

$\gamma$, surface free energy

$H$, Hamaker constant

HEPES, 4-(2-hydroxyethyl)-1-piperazineethanesulfonic acid 
HFIP, 1,1,1,3,3,3-hexafluoro-2-propanol

$\kappa_{\text {eff, }}$, effective indentation constant

$k$, spring constant

$k_{B}$, Boltzmann constant

$\mathrm{MA}$, micropipette aspiration

MAPK, mitogen-activated protein kinase

$m_{\text {eff }}$, mass of the cantilever

MS, microplate stretcher

MT, microtubule

MTC, magnetic twisting cytometry

MTT, 3-(4,5-dimethylthiazol-2-yl)-2,5 diphenyltetrazolium bromide

$v$, Poisson coefficient

$N$, highest harmonic distinguishable about the noise level

$\mathrm{NF}$, neurofilament

NFT, neurofibrillary tangle

OT, optical traps

$P$, area of the power spectrum

PBS, phosphate-buffered saline

PfEMP1, Plasmodium falciparum erythrocyte membrane protein

PID, proportional integral derivative

PIPES, piperazine-N, $\mathrm{N}^{\prime}$-bis(2-ethanesulfonic acid)

$Q$, quality factor of the cantilever

$R_{t i p}$, radius of the cantilever tip

$\mathrm{RBC}$, red blood cell

RFP, red fluorescent protein

$s$, set-point ratio

SPAM, scanning probe acceleration microscopy

SPM, scanning probe microscope

STM, scanning tunneling microscope

$T$, temperature

$t$, time 
TBLE, total brain lipid extract

ThT, thioflavin $\mathrm{T}$

TMAFM, tapping mode atomic force microscopy

$\omega$, drive frequency

$\omega_{o}$, resonance frequency

$\omega_{\text {oper }}$, operating frequency

$x$, cantilever deflection

$y$, cantilever deflection

$y_{\text {rec }}(t)$, reconstructed deflection signal

$z$, position of the cantilever with respect to the surface 


\section{Probing the importance of mechanical properties in biological systems using the atomic force microscope}

Proper cellular functioning is dependent on many processes, ranging from gene expression and signal transduction to the regulation of cell shape, division and migration [1]. All of these processes can be related to the mechanical properties of individual cells and their microenvironments $[2,3]$. The mechanical properties of membranes or whole cells can be influenced by genetics, lifestyle, environmental exposures, and even the normal aging process [4-7]. A growing body of evidence suggests that cellular mechanical disruptions can be directly correlated to a variety of diseases, and understanding the role of mechanical properties in biological systems could be the key to elucidating mechanisms of disease. This introduction provides a brief overview of the importance of biological mechanics, and describes model systems and techniques to evaluate physical and mechanical properties of surfaces, with a particular focus on atomic force microscopy.

\subsection{Alterations in biological mechanics contribute to disease}

Conventionally, researchers have looked to genetics and biochemical events to understand mechanisms of disease. However, advances in cell biology have found that mechanical alterations within a cell and the physical forces acting on a cell can have a profound impact on cellular well-being. These findings led to an emerging field of research, mechanobiology, which investigates how mechanotransduction and modifications to individual cellular mechanical properties contribute to disease [8].

Cells are highly dynamic structural and functional units that all living organisms are composed of, and they are embedded within an extracellular matrix (ECM), consisting of proteoglycans and fibrous proteins [9]. Cells can sense and respond to mechanical forces from the ECM through integrins, adhesion molecules, and cytoskeletal proteins. This process is known as mechanotransduction, and it regulates a many cell functions, some of which include: proliferation, migration and differentiation [10]. There are many diseases which are a result of abnormal mechanotransduction [8].

One such example is breast cancer. In experiments, when mammary epithelial cells were cultured on soft, gel-like substrates, integrins involved in cell signaling are 
activated to promote cell growth by the epidermal growth factor receptor. This model of growth and differentiation is very similar to normal physiological conditions due to the considerable amount of adipose tissue in the breast. However, when the same cells were cultured on rigid surfaces, growth patterns were affected due to the disruption of signaling pathways, which lead to a cancerous phenotype. This phenomenon demonstrates that mechanical properties of the ECM have a significant role in cancer development $[11,12]$, although the exact mechanism of mechanotransduction remains to be elucidated.

Alterations of individual cellular physical and mechanical properties, such as membrane stiffness, elasticity, and adhesion, have also been linked to several diseases. For example, as a cell progresses from normal to cancerous, physical changes of the cytoskeleton occur that promote metastasis. Cancerous mammary cells are notably less rigid than normal cells, which corresponds to a reduction in structural strength [13]. This mechanical transformation is thought to be a result of a reduction of the F-actin network, and that disruption ultimately leads to a decrease in adhesiveness $[11,14,15]$. As the cancer cells transform into an invasive phenotype, mechanical changes become even more pronounced, promoting metastasis.

Contrary to the mechanopathology of breast cancer, red blood cells (RBCs) infected with Plasmodium falciparum, the parasite that causes the symptoms of malaria, experience a significant increase in membrane adhesion. After entering a RBC, the parasite initiates the expression of an erythrocyte membrane protein (PfEMP1) which mediates binding to the endothelium. The intracellular accumulation of PfEMP1 causes the appearance of knob-like structures across the membrane and it also increases membrane stiffness by 10 times the amount of healthy RBCs [16]. Adherence of the rigid and deformed RBCs to the endothelium commonly results in blood clots which can lead to organ failure and death.

Relevant to the studies in this dissertation, slight modifications in membrane cholesterol content, and thereby membrane mechanical properties, can result in severe health consequences throughout the body. Hearing impairments have been linked to membrane cholesterol depletion, which causes an increase in outer hair cell electromechanical response [17]. Heart health can also be compromised, as ion channel 
dysfunction occurred in cardiomyocytes and arteriole smooth muscle cells after membrane cholesterol modulation [18, 19]. Finally, many studies have identified a role for cholesterol-modulated membrane fluidity in the development of Alzheimer's disease [20-22]. The examples presented here represent just a fraction of evidence from the literature acknowledging the critical role of mechanical properties in disease development. This substantiates the need to both study and develop/improve techniques in mechanobiology to more fully understand pathological processes.

\subsection{Model systems to study biological mechanics}

Maintaining cell membrane integrity is critical to proper cellular functioning. The cell membrane provides protection from the extracellular space and regulates process such as cell signaling, ion flux, and adhesion. The membrane is made of a lipid bilayer that is approximately $5 \mathrm{~nm}$ thick (Fig. 1). There are many types of lipids, but they are generally characterized as small, amphiphilic molecules with a hydrophilic head and hydrophobic tail group [23]. The cell membrane contains mainly three categories of lipids: phospholipids, glycolipids, and sterols. In most mammalian cell membranes, the primary lipid components of the bilayer are phospholipids that have a phosphate head group and two hydrocarbon tails. Glycolipids can have many forms but a defining characteristic is the presence of a carbohydrate group [24]. The carbohydrate portion of the lipid typically extends beyond the surface of the bilayer into the extracellular space, where it functions

as a recognition site for chemicals and can help maintain membrane stability via cell to cell attachment. Sterols help maintain membrane stability from within the bilayer and play a role in signaling

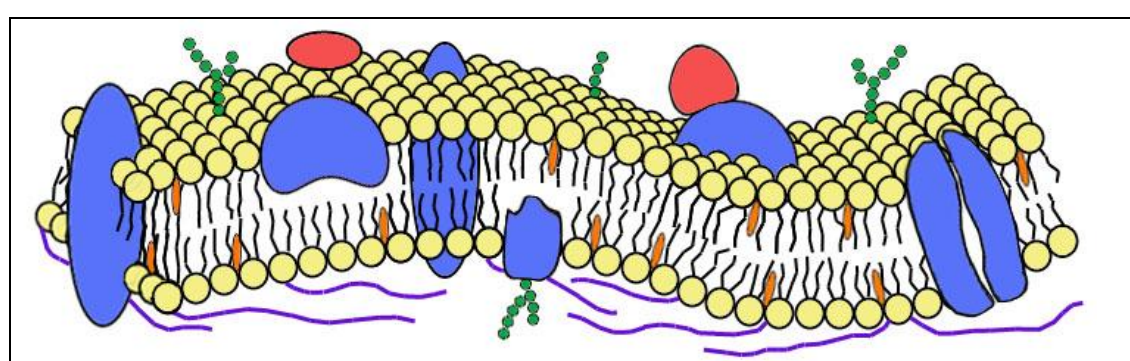

Figure 1. Cross-sectional view of membrane structure and components. A variety of lipids make up the bilayer (phospholipids in yellow, glycolipids with green carbohydrate groups, and sterols in orange), where integral membrane proteins are embedded within the bilayer (partial and transmembrane integral membrane proteins in blue). Above are peripheral membrane proteins (red), and cytoskeletal proteins are depicted below the lipid bilayer (purple). 
[25]. Overall, the lipid composition of a bilayer is highly dependent on the cell type and the location of the membrane, i.e., the plasma membrane verses organelle membranes [26].

A lipid membrane may have various phases, depending on the temperature, pressure or the ratio of lipid components [26]. There are three main phases: solid-ordered (sometimes called the gel phase), liquid-ordered (or liquid crystalline/condensed), and the liquid-disordered (also referred to as the expanded/fluid phase). The solid and liquid phases are determined by the lipid packing on the plane of the membrane, where the solid phase exhibits a lattice arrangement and the liquid phase has no uniform distribution. The ordered and disordered terms refer to the arrangement of lipid chains within the bilayer. The solid ordered phase can be examined experimentally, however, is not believed to exist under physiological conditions [27]. A membrane composed of a single lipid component undergoes a phase transition from an ordered to a disordered state at its melting temperature [27]. Furthermore, membranes can exhibit phase separation, which is easily detected in more complex model systems by the formation of nanoscale domains, or lipid rafts [28-30]. In actual biological membranes, the mixture of lipid components in is far more complex, as there may be hundreds of lipid components within a bilayer. It is likely that there are many coexisting phases in biological membranes, but the appearance of rafts on cellular surfaces has been difficult to observe [31]. For both simple and complex lipid systems, however, the material properties of a membrane such as compressibility and lateral fluidity are highly dependent on the phase of the membrane.

There are several classes of proteins that are affiliated with the lipid bilayer [32]. Peripheral (or extrinsic) membrane proteins interact with bilayer through intermolecular forces between lipids or other membrane bound proteins. They act as scaffolding and can also serve as a site for protein/enzyme attachment or signaling molecules. While peripheral membrane proteins are loosely associated with the lipid bilayer, intrinsic membrane proteins are tightly bound to the lipids, mainly though hydrophobic interactions with the hydrocarbon tails. There are two types of integral (or intrinsic) membrane proteins: those that span the width of the bilayer (transmembrane proteins), and those that are only partially embedded within it. Like phospholipids, integral 
membrane proteins are also amphiphilic. The hydrophilic component(s) protrude(s) from the bilayer to interact with the aqueous environment, while the hydrophobic core is hidden within the bilayer. There are thousands of integral membrane proteins with varying functional roles, including: receptors, transporters, enzymes, adhesion/structural proteins, and many others of uncategorized and unknown functions [33]. Lastly, cytoskeletal proteins are associated with the intracellular portion of the cell membrane. They provide membrane support, contribute to overall cell shape, and are critically important in cellular adhesion (to other cells and to the extracellular matrix). Of these three classes of proteins, only integral membrane proteins directly affect the microstructure of the membrane.

Importantly, the type and arrangement of lipids in a bilayer controls the physicomechanical properties of a membrane, and those properties influence membrane protein functioning. Lipids directly interact with integral membrane proteins through hydrophobic interactions between the hydrocarbon chains of lipid molecules and the hydrophobic portion of the protein. Distortion of the lipid matrix around the protein arises when the lipid-protein hydrophobic regions are mismatched [34, 35]. Hydrophobic mismatching between lipids and proteins is very energetically unfavorable; it can cause the exclusion of certain lipids from the lipid-protein boundary, and if severe enough, can lead to conformational changes in the protein, and aggregation that ultimately alters function [32, 35-38]. Protein conformational changes and aggregation can lead to further membrane disruption. For example, protein modifications can change the lipid packing in the surrounding bilayer, thereby altering bilayer thickness, curvature and stiffness [39, 40]. Thus, the physicomechanical properties of a lipid bilayer play a significant role in the regulation of membrane protein and overall cellular functioning.

The majority of experiments in this dissertation utilized supported lipid bilayers (SLBs) to examine how various factors, such as lipid bilayer composition and temperature, influence the physical and mechanical properties of membranes. Lipid bilayer and vesicle systems have been extensively used in biophysical research for decades. They make an excellent model system for cell surfaces because the influence of single membrane element, molecular/protein interaction, or environmental condition can be studied while avoiding the complexities of cell-based experiments. Specifically, 
bilayers in the described studies were composed of lipid extracts, which more closely resemble cellular conditions as compared to bilayers of a singular lipid composition. In the final experimental chapter, the importance of cellular mechanical properties in relation to Alzheimer's disease was investigated; neurons were modified by disrupting or stabilizing the cytoskeletal protein, tubulin, and the ability of the amyloid- $\beta$ peptide to bind to the cell membrane and cause toxicity was examined.

\subsection{Tools to study biological mechanics}

A variety of techniques can be used to measure lipid membrane or cellular mechanical properties. Langmuir and Langmuir-Blodgett techniques have been commonly used to produce monolayers or multilayers of lipid films for decades. The properties of the lipid film, or the response of film to an external agent, can be studied by creating surface pressure-area isotherms or Brewster angle microscopy [41]. The lipid films are then typically transferred to an atomic force microscope to (AFM) to examine mechanical properties. In AFM, magnetic twisting cytometry (MTC), and nanoindentation, mechanical properties of a sample are calculated by probe-surface interactions [42-47]. Optical traps (OT), microplate stretchers (MS), and micropipette aspiration (MA) can reveal information on lipid vesicle and cellular mechanics by stretching the surface between beads (OT), glass plates (MS), or into a pipette (MA) [48-55]. Although all of these techniques have proven to be useful in gaining mechanical information, AFM offers the unique advantage of simultaneously capturing nanoscale resolution 3D topographic images and mechanical surface maps. Information that can be gained from AFM includes: topographic measurements, phase contrast, stiffness, viscoelasticity, adhesion, and frictional forces. A benefit of AFM-based mechanical investigations is that one can measure localized mechanical properties on the nanoscale, whereas most of the other previously mentioned techniques can only perform mechanical evaluations on micronsized areas of the membrane, or over the whole lipid vesicle/cell. This feature is especially useful when attempting to understand how smaller objects, such as nanoparticles or proteins, affect membrane mechanics. AFM is also particularly well suited for biological investigations because the forces with which the cantilever tip probes the surface are nondestructive (commonly in the $\mathrm{nN}$ range), thereby prolonging 
membrane viability/sample integrity [56]. Furthermore, sample preparation is minimal and membranes or whole cells can be imaged under near-physiological conditions (temperature, $\mathrm{pH}$ ). In this work, several AFM-based techniques were used to map the physical and material properties of supported lipid bilayers and whole cells.

\subsection{Development and operational principles of AFM}

AFM has been used extensively to study both the morphological and material properties of a variety of biological samples ranging from DNA and proteins to whole cells and tissues [57]. Herein, the development of AFM and the principles behind the various modes of operation are described.

\subsubsection{History and fundamentals of the atomic force microscope (AFM)}

Scanning probe microscopes (SPM) produce images by scanning a physical probe across a sample surface. Unlike light-based microscopes, SPMs "feel" rather than "see" a surface through the interaction between the probe tip and the sample surface. The first SPM, the scanning tunneling microscope (STM), was developed in 1981 by two scientists at IBM, Binnig and Rohrer [57]. The operational principle of STM is based on the tunneling effect of quantum mechanics. In STM, a metallic tip is raster scanned above a sample surface, and when an electric voltage is applied, tunneling occurs, and the tunneling current is plotted as a function of tip position to produce a surface image. STM is capable of achieving atomic resolution, however there are a few major limitations. First, STM requires conductive or semiconductive samples for the tunneling phenomena to occur, and secondly, it is not possible to obtain information about the material properties of a sample because the probe tip never contacts the surface [58]. The atomic force microscope (AFM) was developed by Binnig in 1986 to overcome these limitations [59].

The AFM can be operated under a variety of conditions: one can examine rigid or soft materials in air or liquid at various pressures and temperatures. True atomic resolution can be achieved under certain conditions, although nanoscale spatial resolution is more common, and scanning areas can be as large as $100 \mu \mathrm{m}$ with a vertical range of approximately $15 \mu \mathrm{M}[57,60]$. More sophisticated instruments are equipped with 


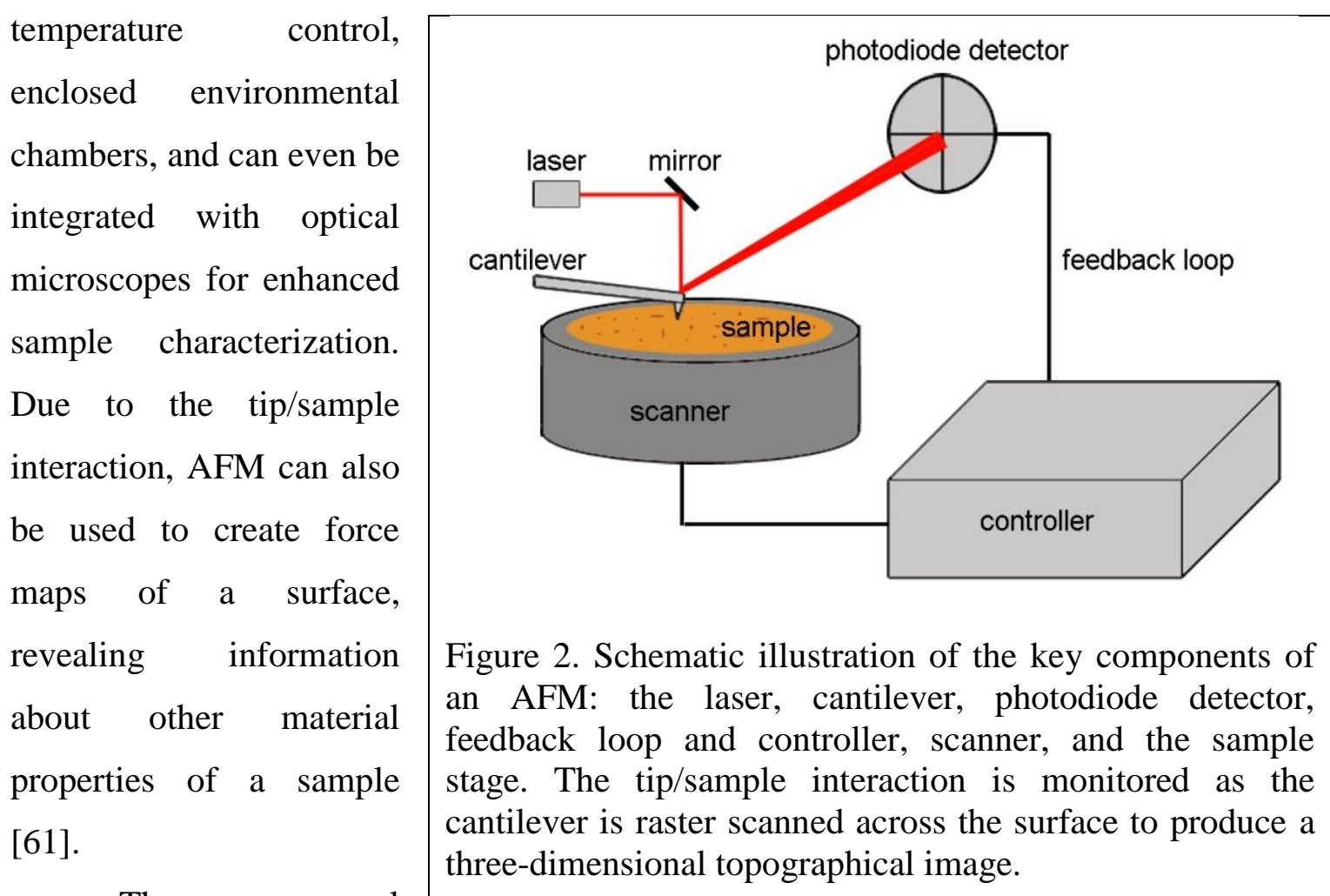

There are several

key operational components of an AFM: the laser, cantilever, photodiode detector, the feedback loop and controller, scanner, and the sample stage (Fig. 2) [58]. The cantilever is considered to be the heart of AFM because images are constructed by monitoring the interaction between its tip and the surface. Typical cantilevers are made of silicon or silicon nitride, and sometimes have a thin metallic coating to improve reflectivity. There are two standard shapes of cantilevers: triangular or rectangular, which is also known as a "diving board" shape [62]. Commonly, the tip of the cantilever has a pyramidal shape with a radius ranging from 10 to $50 \mathrm{~nm}$. The dimensions (such as length, width, and thickness) determine the spring constant and resonance frequency, two factors which must be considered when choosing a cantilever [63]. The discussion of how cantilever properties correlate to imaging forces will be expanded in later Chapter 2.

AFMs utilize an "optical lever" system, where a laser beam is reflected off the back of a cantilever onto a photodiode detector, typically four-sectored. Displacement of the cantilever alters the trajectory of the beam, thereby repositioning the laser on the detector. The difference between the photodiode signals is sent through an open feedback loop, which enables the user to control the imaging conditions. The resultant signal is 
transmitted to the scanner, which is commonly made of a piezoelectric crystals that expand or contract based on the signal. This action raises or lowers the sample stage to track the surface morphology without damaging the cantilever tip. A three dimensional image is produced by storing the data in an array, typically with 128 to 1024 points per scan line [64].

The described AFM system is what is known as the scanned-sample AFM, where the scanner is positioned directly below the sample stage. Here, the cantilever tip is held in a fixed position, and the scanner controls the $x, y$, and $z$ movement of the sample during imaging. Because the scanner is separate from the cantilever, the mechanical noise of the system is very low, resulting in excellent spatial resolution. In this work, a scanned-sample AFM was used.

AFM is most commonly used as an imaging tool, and there are two main AFM imaging modes: static and dynamic. Static mode AFM, also known as contact mode, monitors the static deflection of the cantilever. Alternatively, dynamic mode AFM monitors the oscillation amplitude of a driven cantilever. Dynamic mode AFM can be subcategorized into tapping mode and noncontact mode. Although useful for imaging delicate materials due to its nondestructive nature, noncontact mode was not used for these studies because mechanical information cannot be obtained due to the fact that the tip never contacts the surface, and experiments in this mode are typically performed in vacuum. In addition to AFM imaging techniques, this work also employed several AFMbased methods to correlate tip/sample forces to localized material properties of a sample. These techniques included: phase contrast imaging, higher harmonic imaging, forcedistance curves, force volume imaging, and scanning probe acceleration microscopy. The following sections give a brief overview of the image acquisition processes, and also how mechanical information was acquired.

\subsubsection{Contact mode AFM}

In contact mode AFM (CMAFM) the cantilever deflection signal is monitored to produce an image. To begin the imaging process, the cantilever tip is lowered into contact with the sample, and it is essentially dragged across the sample surface during scanning. The tip position is altered to track the surface morphology while the closed feedback loop 
maintains a constant deflection. The amount of cantilever deflection is proportional to the applied force, hence, contact mode AFM is sometimes also referred to as constant force mode. The force-deflection relationship can be described by Hooke's Law,

$$
F=-k x
$$

where $F$ is the applied force, $k$ is the cantilever spring constant and $x$ is the cantilever deflection. Contact mode can produce images with excellent spatial resolution, but the repulsive forces experienced between the tip and sample can be damaging to delicate surfaces [65]. To circumvent this issue, it is important to use cantilevers with low spring constants when imaging any soft or biological samples to reduce the imaging force. CMAFM has successfully been applied to image many biological samples, and some interesting applications included: experiments to track the aggregation of membrane sheets in response to $\mathrm{pH}$ changes, the appearance of granular particles or proteins on cell membranes, and the disaggregation of the actin network after whole living cells were exposed to cytoskeletal modifying treatments [66-68]. Thus, even though the technique is preferred for more rigid samples due to the dragging action of the tip, by controlling the tip/sample interaction forces, it is possible to image delicate samples with excellent spatial and temporal resolution.

\subsubsection{Tapping mode AFM}

Tapping mode AFM (TMAFM) is a widely used dynamic mode technique that obtains topographic images of surfaces by monitoring the oscillation amplitude of a cantilever with an ultrasharp tip probe that physically interacts with the surface. In this imaging mode, the cantilever is commonly driven near its resonance frequency, $\omega_{o}$, by a piezoelectric bimorph element mounted near the base of the cantilever. The cantilever tip is then lowered into close proximity with the sample and allowed to intermittently contact the surface. The contact leads to a decrease of the cantilever oscillation amplitude from the "free" amplitude, $A_{\mathrm{o}}$, to a tapping amplitude, $A$ [69]. On rigid surfaces, the cantilever oscillation amplitude decreases linearly as the distance between the tip and the sample, $D_{0}$, becomes smaller. As a result, surface topography is mapped by raster scanning the probe across the surface (in the $x y$ direction) while using a closed feedback loop to continuously adjust the vertical (in the $z$ direction) extension of a piezoelectric 
scanner to maintain a constant tapping amplitude or set-point ratio, $s=A / A_{0}$. In these studies, TMAFM was used as the main imaging technique, because it provides the basis for scanning probe acceleration microscopy experiments, and it is less damaging to biological samples than CMAFM.

\subsubsection{Phase contrast imaging}

During the TMAFM imaging process, the phase signal of the cantilever can be simultaneously recorded to produce a phase contrast image. A shift in the phase signal occurs when there is a phase lag between the cantilever oscillation signal relative to the driving signal (Fig. 3). The phase lag is extremely sensitive to any changes in surface properties, such as stiffness, producing images with excellent compositional contrast. While monitoring the phase of the cantilever in tapping mode is a common method to acquire some insight into the mechanical properties of surfaces, such images can be difficult to interpret due to various causes of energy dissipation, including capillary forces, viscoelasticity of the sample, cross talk with topography, and frictional forces associated with the tilt of the cantilever and/or surface [70-75]. Furthermore, the phase behavior of the cantilever becomes increasingly complicated when imaging in solution. For example, the phase contrast associated with soft cantilevers imaging in solution can arise from either (i) energy propagation during the tapping event that excites higher eigenmodes of the cantilever mediated by short-range interactions, or (ii) tip/sample energy dissipation [76]. Due to these difficulties, phase imaging is generally applied as a complementary technique. Chapter 4 demonstrates how phase imaging can be used in combination with (a)

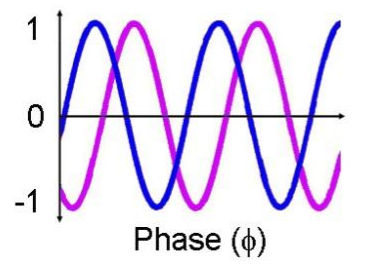

(c)

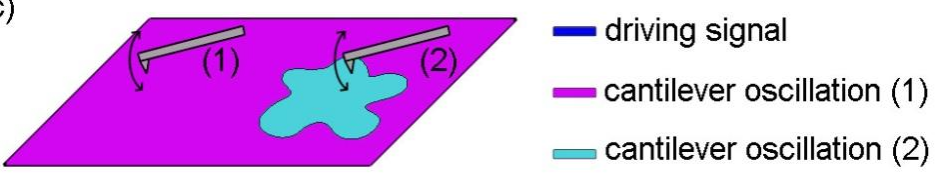

Figure 3. Principle of phase contrast imaging. The phase signal is sensitive to changes in compositional contrast of surfaces. (a-b) Demonstrates the phase lag between the cantilever driving signal (dark blue) and the cantilever oscillation signal on (c) two different surfaces (region 1, purple vs. region 2 light blue). 
TMAFM, higher harmonic imaging, and force reconstruction techniques to gain a more complete understanding of surface physicomechanical properties.

\subsubsection{Higher harmonic imaging}

Oscillation amplitude and phase signals are commonly acquired during standard TMAFM operation, but additional information about sample properties is hidden within the vibrational frequencies of the cantilever. During TMAFM imaging, nonlinear tip/sample interactions cause excitation of higher frequencies that are harmonic to the driving frequency. By capturing the entire cantilever deflection trajectory during TMAFM imaging, higher harmonic signals can be extracted to produce images corresponding to individual harmonic frequencies. Like phase imaging, higher harmonic imaging is a supplementary technique that can provide insight on the material properties of a sample surface. The theory of higher harmonic imaging and experiments are further discussed in Chapter 4.

\subsubsection{Force-distance curves and force volume imaging}

The construction of force-distance curves and force volume imaging are AFM-based techniques that have proven to be very useful in determining the material properties of biological surfaces. These methods are utilized in the vast majority of AFM-based mechanical studies on biological surfaces. For example, force volume imaging has been successfully applied to study bacterial surfaces, examining mechanical differences between human cells with benign and malignant phenotypes, and microdomains within lipid bilayers [15, 44, 77]. There are two regimes of the force-distance curve which inform us about surface mechanics, (a) the attractive regime (negative forces) and (b) the repulsive regime (positive forces) (Fig. 4). On the approach, the cantilever tip is lowered to the sample surface, and snaps into contact due to the attractive forces between the tip and the surface contact. When the deflection reaches its maximum, the tip begins to retract to its original position, during which hysteresis may be experienced due to tip/sample adhesion. The tip can then be moved to the next desired $x y$ coordinate to produce another curve. Force-distance curves can reveal information about energy 
dissipation, stiffness (Young's Modulus), and the adhesion between the cantilever tip and sample [62].

Force volume imaging, can be used to create a mechanical map of an entire sample surface by creating an array of force-distance curves, rather than just a few selected coordinates. Although force volume imaging has proven to be valuable in gaining mechanical insights biological surfaces, it is limited in that spatial resolution must be sacrificed for temporal resolution. Common pixel resolution for AFM imaging is 512 x 512, and typical force curves run at a rate of $1 \mathrm{~Hz}$.

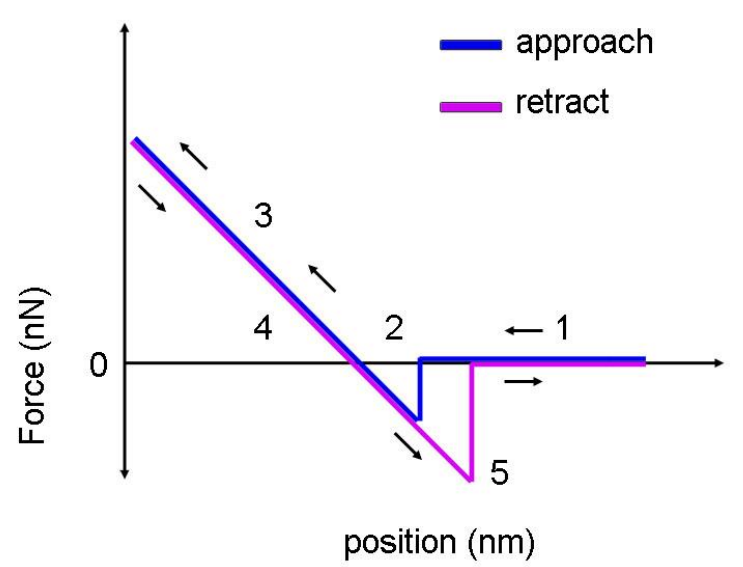

Figure 4. Illustration of a force-distance curve. To create a force distance-curve, the cantilever is lowered to the sample (1), where it snaps into contact with the surface due to the attractive forces between the tip and the surface (2). The cantilever pushes into the surface (3) until it reaches a maximum deflection, and begins to retract (4). Hysteresis may be observed due to adhesion between the tip and the sample (5) until the tip and sample become detached.

Therefore, it would take slightly over three days to create a force volume image of that resolution, and when working with live biological materials, it is essential to quickly make measurements to preserve membrane integrity (avoid sample drying or the lateral growth of a membrane patch into a complete bilayer).

To evaluate force-distance curves and force volume images, it is critical to know properties of the cantilever, including the deflection sensitivity and the spring constant. The deflection sensitivity parameter is used to convert deflection (recorded in volts) to nanometers so that the force can be calculated. Deflection sensitivity is calculated prior to each experiment by taking a force-distance curve on a hard substrate, such as mica. Once the deflection sensitivity is known, thermal tuning is performed to determine the spring constant. The principle of thermal tuning is that the cantilever fluctuates in response to thermal noise, and the spring constant of the cantilever is measured by relating the displacement of the lever as a function of time to the frequency spectrum of the fluctuations (which is proportional to the power spectral density). Once the spring 
constant is calculated, the mass of the cantilever can be determined by the following relationships:

$$
k=\frac{k_{B} T}{P} \quad \text { and } \quad m=\frac{k}{\omega_{o}^{2}},
$$

where $k$ is the spring constant, $k_{B}$ is the Boltzmann constant, $T$ is the temperature, $P$ is the area of the power spectrum, $\omega_{o}$ is the cantilever's resonance frequency and $m$ is the mass of the cantilever $[62,78,79]$. When comparing material properties across experiments, it is critical to properly calibrate the cantilever for accurate comparisons. This is true for force-distance curves, force volume imaging, and scanning probe acceleration microscopy studies.

\subsubsection{Scanning probe acceleration microscopy}

In many cases, it is desirable to simultaneously map the topographical and mechanical properties of sample surfaces with high spatial resolution. Recent advances in reconstructing the time-resolved tip/sample force during the acquisition of a tapping mode image have provided further access to information relating to the mechanical properties of surfaces. An advantage of such force reconstruction approaches is that they allow for the direct correlation of topographic features with relative changes in mechanical properties. One of the traditional advantages of TMAFM is its ability to image surfaces in solution, and scanning probe acceleration microscopy (SPAM) is a method capable of reconstructing the time-resolved tip/sample forces of solution-based TMAFM experiments. This feature of SPAM makes it a well-suited technique for the biological investigations described in this dissertation [28, 80-86].

SPAM was developed at Carnegie Mellon University in the lab of Tomasz Kowalewski. The theory behind the technique is that the cantilever is treated as an accelerometer and cantilever acceleration can be directly related to the tip/sample force by taking the second derivative of the cantilever deflection signal [80]. As a result, this technique does not require specially designed cantilevers, and it can be applied to any AFM with the addition of a signal access module, which used to capture the cantilever deflection trajectory during TMAFM imaging. The following chapter thoroughly describes the use of time-resolved tip/sample forces to obtain insight into the mechanical properties of surfaces, with a focus on the SPAM technique. Chapters 3 and 4 
demonstrate the ability of SPAM to track mechanical changes in supported lipid bilayers due to alterations in membrane cholesterol content and temperature, respectively.

\subsection{References}

1. Kamm, R., J. Lammerding, and M. Mofrad, Cellular Nanomechanics, in Springer Handbook of Nanotechnology, B. Bhushan, Editor 2010, Springer: New York, NY. p. 1171-1200.

2. Ingber, D.E., Tensegrity: The architectural basis of cellular mechanotransduction. Annual Review of Physiology, 1997. 59: p. 575-599.

3. Chicurel, M.E., C.S. Chen, and D.E. Ingber, Cellular control lies in the balance of forces. Current Opinion in Cell Biology, 1998. 10(2): p. 232-239.

4. Jacobs, C.R., S. Temiyasathit, and A.B. Castillo, Osteocyte mechanobiology and pericellular mechanics, in Annual Review of Biomedical Engineering, Vol 12, M.L. Yarmush, J.S. Duncan, and M.L. Gray, Editors. 2010. p. 369-400.

5. Gourlay, C.W. and K.R. Ayscough, The actin cytoskeleton in ageing and apoptosis. FEMS Yeast Research, 2005. 5(12): p. 1193-1198.

6. Lacolley, P., P. Challande, M. Osborne-Pellegrin, and V. Regnault, Genetics and pathophysiology of arterial stiffness. Cardiovascular Research, 2009. 81(4): p. 637-648.

7. Nash, G.B., E. Obrien, E.C. Gordonsmith, and J.A. Dormandy, Abnormalities in the mechanical properties of RBCs caused by Plasmodium Falciparum. Blood, 1989. 74(2): p. 855-861.

8. Ingber, D.E., Mechanobiology and diseases of mechanotransduction. Annals of Medicine, 2003. 35(8): p. 564-577.

9. Bosman, F.T. and I. Stamenkovic, Functional structure and composition of the extracellular matrix. Journal of Pathology, 2003. 200(4): p. 423-428.

10. Lukashev, M.E. and Z. Werb, ECM signalling: orchestrating cell behaviour and misbehaviour. Trends in Cell Biology, 1998. 8(11): p. 437-441.

11. Kumar, S. and V. Weaver, Mechanics, malignancy, and metastasis: The force journey of a tumor cell. Cancer and Metastasis Reviews, 2009. 28(1-2): p. 113127. 
12. Suresh, S., Biomechanics and biophysics of cancer cells. Acta Biomaterialia, 2007. 3(4): p. 413-438.

13. Guck, J., S. Schinkinger, B. Lincoln, F. Wottawah, S. Ebert, M. Romeyke, D. Lenz, H.M. Erickson, R. Ananthakrishnan, D. Mitchell, J. Kas, S. Ulvick, and C. Bilby, Optical deformability as an inherent cell marker for testing malignant transformation and metastatic competence. Biophysical Journal, 2005. 88(5): p. 3689-3698.

14. Katsantonis, J., A. Tosca, S.B. Koukouritaki, P.A. Theodoropoulos, A. Gravanis, and C. Stournaras, Differences in the G/total actin ratio and microfilament stability between normal and malignant human keratinocytes. Cell Biochemistry and Function, 1994. 12(4): p. 267-274.

15. Cross, S.E., Y.S. Jin, J. Rao, and J.K. Gimzewski, Nanomechanical analysis of cells from cancer patients. Nature Nanotechnology, 2007. 2(12): p. 780-783.

16. Lim, C.T. and A. Li, Mechanopathology of red blood cell diseases - Why mechanics matters. Theoretical and Applied Mechanics Letters, 2011. 1(1): p. -.

17. Brownell, W.E., S. Jacob, P. Hakizimana, M. Ulfendahl, and A. Fridberger, Membrane cholesterol modulates cochlear electromechanics. Pflugers ArchivEuropean Journal of Physiology, 2011. 461(6): p. 677-686.

18. Gleason, M.M., M.S. Medow, and T.N. Tulenko, Excess membrane cholesterol alters calcium movements, cytosolic calcium levels, and membrane fluidity in arterial smooth-muscle cells. Circulation Research, 1991. 69(1): p. 216-227.

19. Abi-Char, J., A. Maguy, A. Coulombe, E. Balse, P. Ratajczak, J.L. Samuel, S. Nattel, and S.N. Hatem, Membrane cholesterol modulates Kv1.5 potassium channel distribution and function in rat cardiomyocytes. Journal of PhysiologyLondon, 2007. 582(3): p. 1205-1217.

20. Yip, C.M., E.A. Elton, A.A. Darabie, M.R. Morrison, and J. McLaurin, Cholesterol, a modulator of membrane-associated A $\beta$-fibrillogenesis and neurotoxicity. Journal of Molecular Biology, 2001. 311(4): p. 723-734.

21. Ji, S.R., Y. Wu, and S.F. Sui, Cholesterol is an important factor affecting the membrane insertion of $\beta$-amyloid peptide (A $\beta 1-40)$, which may potentially inhibit the fibril formation. Journal of Biological Chemistry, 2002. 277(8): p. 6273-6279. 
22. Arispe, N. and M. Doh, Plasma membrane cholesterol controls the cytotoxicity of Alzheimer's disease A $\beta P(1-40)$ and (1-42) peptides. Faseb Journal, 2002. 16(12): p. 1526-1536.

23. Singer, S.J. and G.L. Nicolson, The fluid mosaic model of the structure of cell membranes. Science, 1972. 175(4023): p. 720-731.

24. Curatolo, W., The physical properties of glycolipids. Biochimica Et Biophysica Acta, 1987. 906(2): p. 111-136.

25. Bloch, K.E., Sterol structure and membrane function. Crc Critical Reviews in Biochemistry, 1983. 14(1): p. 47-92.

26. Heimburg, T., Physical properties of biological membranes, in Handbook of Molecular Biophysics, H.G. Bohr, Editor 2009, Wiley: Copenhagen. p. 593-616.

27. Connell, S.D. and D.A. Smith, The atomic force microscope as a tool for studying phase separation in lipid membranes (Review). Molecular Membrane Biology, 2006. 23(1): p. 17-28.

28. Shamitko-Klingensmith, N., K.M. Molchanoff, K.A. Burke, G.J. Magnone, and J. Legleiter, Mapping the mechanical properties of cholesterol-containing supported lipid bilayers with nanoscale spatial resolution. Langmuir, 2012. 28(37): p. 13411-13422.

29. Shamitko-Klingensmith, N. and J. Legleiter, Investigation of temperature induced mechanical changes in supported bilayers by variants of tapping mode atomic force microscopy. Scanning, 2014: p. n/a-n/a.

30. Henderson, R.M., J.M. Edwardson, N.A. Geisse, and D.E. Saslowsky, Lipid Rafts: Feeling is Believing. Vol. 19. 2004. 39-43.

31. Gaus, K., E. Gratton, E.P.W. Kable, A.S. Jones, I. Gelissen, L. Kritharides, and W. Jessup, Visualizing lipid structure and raft domains in living cells with twophoton microscopy. Proceedings of the National Academy of Sciences, 2003. 100(26): p. 15554-15559.

32. Nicolson, G.L., The fluid-mosaic model of membrane structure: still relevant to understanding the structure, function and dynamics of biological membranes after more than 40 years. Biochimica Et Biophysica Acta-Biomembranes, 2014. 1838(6): p. 1451-1466. 
33. Almen, M.S., K.J.V. Nordstrom, R. Fredriksson, and H.B. Schioth, Mapping the human membrane proteome: a majority of the human membrane proteins can be classified according to function and evolutionary origin. Bmc Biology, 2009. 7.

34. Mouritsen, O.G. and M. Bloom, Mattress model of lipid-protein interactions in membranes. Biophysical Journal, 1984. 46(2): p. 141-153.

35. Mouritsen, O.G., Lipids, curvature, and nano-medicine. European Journal of Lipid Science and Technology, 2011. 113(10): p. 1174-1187.

36. Kusumi, A., K.G.N. Suzuki, R.S. Kasai, K. Ritchie, and T.K. Fujiwara, Hierarchical mesoscale domain organization of the plasma membrane. Trends in Biochemical Sciences, 2011. 36(11): p. 604-615.

37. Dumas, F., M.C. Lebrun, and J.F. Tocanne, Is the protein/lipid hydrophobic matching principle relevant to membrane organization and functions? Febs Letters, 1999. 458(3): p. 271-277.

38. Kusumi, A. and J.S. Hyde, Spin-label saturation-transfer electron-spin resonance detection of transient association of rhodopsin in reconstituted membranes. Biochemistry, 1982. 21(23): p. 5978-5983.

39. Andersen, O.S. and R.E. Koeppe, Bilayer Thickness and Membrane Protein Function: An Energetic Perspective. Annual Review of Biophysics and Biomolecular Structure, 2007. 36(1): p. 107-130.

40. Lundbaek, J.A., S.A. Collingwood, H.I. Ingolfsson, R. Kapoor, and O.S. Andersen, Lipid bilayer regulation of membrane protein function: gramicidin channels as molecular force probes. Journal of the Royal Society Interface, 2010. 7(44): p. 373-395.

41. Torrent-Burgues, J., G. Oncins, and F. Sanz, Nanomechanics of LangmuirBlodgett films. Contributions to Science, 2009. 4(2): p. 177-186.

42. Laurent, V.r.M., S. Hénon, E. Planus, R. Fodil, M. Balland, D. Isabey, and F.o. Gallet, Assessment of mechanical properties of adherent living cells by bead micromanipulation: comparison of magnetic twisting cytometry vs optical tweezers. Journal of Biomechanical Engineering, 2002. 124(4): p. 408-421. 
43. Picas, L., F. Rico, and S. Scheuring, Direct measurement of the mechanical properties of lipid phases in supported bilayers. Biophysical Journal, 2012. 102(1): p. L1-L3.

44. An, H., M.R. Nussio, M.G. Huson, N.H. Voelcker, and J.G. Shapter, Material Properties of Lipid Microdomains: Force-Volume Imaging Study of the Effect of Cholesterol on Lipid Microdomain Rigidity. Biophysical Journal. 99(3): p. 834844.

45. Steltenkamp, S., M.M. Muller, M. Deserno, C. Hennesthal, C. Steinem, and A. Janshoff, Mechanical properties of pore-spanning lipid bilayers probed by atomic force microscopy. Biophysical Journal, 2006. 91(1): p. 217-226.

46. Alessandrini, A., H.M. Seeger, T. Caramaschi, and P. Facci, Dynamic Force Spectroscopy on Supported Lipid Bilayers: Effect of Temperature and Sample Preparation. Biophysical Journal, 2012. 103(1): p. 38-47.

47. Wang, N. and D.E. Ingber, Probing transmembrane mechanical coupling and cytomechanics using magnetic twisting cytometry. Biochemistry and Cell Biology-Biochimie Et Biologie Cellulaire, 1995. 73(7-8): p. 327-335.

48. Huang, H.D., R.D. Kamm, and R.T. Lee, Cell mechanics and mechanotransduction: pathways, probes, and physiology. American Journal of Physiology-Cell Physiology, 2004. 287(1): p. C1-C11.

49. Longo, M. and H. Ly, Micropipet aspiration for measuring elastic properties of lipid bilayers, in Methods in Membrane Lipids, A. Dopico, Editor 2007, Humana Press. p. 421-437.

50. Needham, D. and R.S. Nunn, Elastic deformation and failure of lipid bilayer membranes containing cholesterol. Biophysical Journal, 1990. 58(4): p. 9971009.

51. Olbrich, K., W. Rawicz, D. Needham, and E. Evans, Water permeability and mechanical strength of polyunsaturated lipid bilayers. Biophysical Journal, 2000. 79(1): p. 321-327.

52. Solmaz, M.E., R. Biswas, S. Sankhagowit, J.R. Thompson, C.A. Mejia, N. Malmstadt, and M.L. Povinelli, Optical stretching of giant unilamellar vesicles 
with an integrated dual-beam optical trap. Biomedical Optics Express, 2012. 3(10): p. 2419-2427.

53. Pinon, T.M., L.S. Hirst, and J.E. Sharping. Fiber-based dual-beam optical trapping system for studying lipid vesicle mechanics. in Optics in the Life Sciences. 2011. Monterey, California: Optical Society of America.

54. Qian, F., S. Ermilov, D. Murdock, W.E. Brownell, and B. Anvari, Combining optical tweezers and patch clamp for studies of cell membrane electromechanics. Review of Scientific Instruments, 2004. 75(9): p. 2937-2942.

55. Ermilov, S.A., D.R. Murdock, F. Qian, W.E. Brownell, and B. Anvari, Studies of plasma membrane mechanics and plasma membrane-cytoskeleton interactions using optical tweezers and fluorescence imaging. Journal of Biomechanics, 2007. 40(2): p. 476-480.

56. Sokolov, I., Atomic force microscopy in cancer cell research, in Cancer Nanotechnology, H.S. Nalwa and T. Webster, Editors. 2007, American Scientific Publishers Inc. p. 1-17.

57. Allison, D.P., N.P. Mortensen, C.J. Sullivan, and M.J. Doktycz, Atomic force microscopy of biological samples. Wiley Interdisciplinary ReviewsNanomedicine and Nanobiotechnology, 2010. 2(6): p. 618-634.

58. Binnig, G., C.F. Quate, and C. Gerber, Atomic force microscope. Physical Review Letters, 1986. 56(9): p. 930-933.

59. Binnig, G., Atomic force microscope and method for imaging surfaces with atomic resolution, 1988, International Business Machines Corporation: United States.

60. Boneschanscher, M.P., J. van der Lit, Z. Sun, I. Swart, P. Liljeroth, and D. Vanmaekelbergh, Quantitative atomic resolution force imaging on epitaxial graphene with reactive and nonreactive AFM probes. ACS Nano, 2012. 6(11): p. 10216-10221.

61. Jalili, N. and K. Laxminarayana, A review of atomic force microscopy imaging systems: application to molecular metrology and biological sciences. Mechatronics, 2004. 14(8): p. 907-945. 
62. Cappella, B. and G. Dietler, Force-distance curves by atomic force microscopy. Surface Science Reports, 1999. 34(1-3).

63. Tortonese, M., Cantilevers and tips for atomic force microscopy. Ieee Engineering in Medicine and Biology Magazine, 1997. 16(2): p. 28-33.

64. Baselt, D.R., S.M. Clark, M.G. Youngquist, C.F. Spence, and J.D. Baldeschwieler, Digital signal processor control of scanned probe microscopes. Review of Scientific Instruments, 1993. 64(7): p. 1874-1882.

65. Butt, H.J., P. Siedle, K. Seifert, K. Fendler, T. Seeger, E. Bamberg, A.L. Weisenhorn, K. Goldie, and A. Engel, Scan speed limit in atomic force microscopy. Journal of Microscopy, 1993. 169(1): p. 75-84.

66. Le Grimellec, C., E. Lesniewska, M.-C. Giocondi, E. Finot, V. Vié, and J.-P. Goudonnet, Imaging of the surface of living cells by low-force contact-mode atomic force microscopy. Biophysical Journal, 1998. 75(2): p. 695-703.

67. Rotsch, C. and M. Radmacher, Drug-induced changes of cytoskeletal structure and mechanics in fibroblasts: An atomic force microscopy study. Biophysical Journal, 2000. 78(1): p. 520-535.

68. Mueller, D.J., F.A. Schabert, G. Bueldt, and A. Engel, Imaging purple membranes in aqueous solutions at sub-nanometer resolution by atomic force microscopy. Biophysical Journal, 1995. 68(5): p. 1681-1686.

69. Burnham, N.A., O.P. Behrend, F. Oulevey, G. Gremaud, P.J. Gallo, D. Gourdon, E. Dupas, A.J. Kulik, H.M. Pollock, and G.A.D. Briggs, How does a tip tap? Nanotechnology, 1997. 8(2): p. 67-75.

70. Cleveland, J.P., B. Anczykowski, A.E. Schmid, and V.B. Elings, Energy dissipation in tapping-mode atomic force microscopy. Applied Physics Letters, 1998. 72(20): p. 2613-2615.

71. Tamayo, J. and R. Garcia, Relationship between phase shift and energy dissipation in tapping-mode scanning force microscopy. Applied Physics Letters, 1998. 73(20): p. 2926-2928.

72. Zitzler, L., S. Herminghaus, and F. Mugele, Capillary forces in tapping mode atomic force microscopy. Physical Review B, 2002. 66(15). 
73. Garcia, R., J. Tamayo, and A. San Paulo, Phase contrast and surface energy hysteresis in tapping mode scanning force microsopy. Surface and Interface Analysis, 1999. 27(5-6): p. 312-316.

74. Stark, M., C. Moller, D.J. Muller, and R. Guckenberger, From images to interactions: High-resolution phase imaging in tapping-mode atomic force microscopy. Biophysical Journal, 2001. 80(6): p. 3009-3018.

75. Marcus, M.S., R.W. Carpick, D.Y. Sasaki, and M.A. Eriksson, Material anisotropy revealed by phase contrast in intermittent contact atomic force microscopy. Physical Review Letters, 2002. 88(22).

76. Melcher, J., C. Carrasco, X. Xu, J.L. Carrascosa, J. Gomez-Herrero, P.J. de Pablo, and A. Raman, Origins of phase contrast in the atomic force microscope in liquids. Proceedings of the National Academy of Sciences of the United States of America, 2009. 106(33): p. 13655-13660.

77. Gaboriaud, F., B.S. Parcha, M.L. Gee, J.A. Holden, and R.A. Strugnell, Spatially resolved force spectroscopy of bacterial surfaces using force-volume imaging. Colloids and Surfaces B: Biointerfaces, 2008. 62(2): p. 206-213.

78. Hutter, J.L. and J. Bechhoefer, Calibration of atomic force microscope tips. Review of Scientific Instruments, 1993. 64(11): p. 3342-3342.

79. Foster, B., New atomic force microscopy (AFM) approaches life sciences gently, quantitatively, and correlatively. American Laboratory, 2012. 44(4).

80. Legleiter, J., M. Park, B. Cusick, and T. Kowalewski, Scanning probe acceleration microscopy (SPAM) in fluids: Mapping mechanical properties of surfaces at the nanoscale. Proceedings of the National Academy of Sciences of the United States of America, 2006. 103(13): p. 4813-4818.

81. Legleiter, J., J.D. Fryer, D.M. Holtzman, and T. Kowalewski, The modulating effect of mechanical changes in lipid bilayers caused by ApoE-containing lipoproteins on amyloid $\beta$ induced membrane disruption. Acs Chemical Neuroscience, 2011. 2(10): p. 588-599.

82. Yates, E.A., S.L. Owens, M.F. Lynch, E.M. Cucco, C.S. Umbaugh, and J. Legleiter, Specific domains of amyloid $\beta$ facilitate aggregation on and association with lipid bilayers. Journal of Molecular Biology, 2013. 425(11): p. 1915-1933. 
83. Chaibva, M., K.A. Burke, and J. Legleiter, Curvature enhances binding and aggregation of Huntingtin at lipid membranes. Biochemistry, 2014. 53(14): p. 2355-2365.

84. Burke, K.A., E.A. Yates, and J. Legeiter, Amyloid-forming proteins alter the local mechanical properties of lipid membranes. Biochemistry, 2013. 52(5): p. 808817.

85. Burke, K.A., K.M. Hensal, C.S. Umbaugh, M. Chaibva, and J. Legleiter, Huntingtin disrupts lipid bilayers in a polyQ-length dependent manner. Biochimica et Biophysica Acta (BBA) - Biomembranes, 2013. 1828(8): p. 19531961.

86. Burke, K.A., K.J. Kauffman, C.S. Umbaugh, S.L. Frey, and J. Legleiter, The interaction of polyglutamine peptides with lipid membranes is regulated by flanking sequences associated with Huntingtin. Journal of Biological Chemistry, 2013. 288(21): p. 14993-15005. 


\section{Scanning probe acceleration microscopy: theory and experiments ${ }^{1}$}

Techniques that quantitatively and temporally monitor, track, and/or characterize the physical and material properties of biologically relevant surfaces with nanoscale spatial resolution are highly desirable. Advances in the field of scanning probe microscopy have made it possible to simultaneously track morphology and material surface properties in a non-invasive manner under physiologically relevant conditions. One such technique is scanning probe acceleration microscopy (SPAM), which is based on tapping mode atomic force microscopy (AFM). With SPAM, it is possible to study the material properties of a sample surface with nanoscale spatial resolution by reconstructing the time-resolved tip/sample force interaction during individual tapping events of the tapping mode AFM imaging process in solution. Due to its basis is solution tapping mode AFM, SPAM has the potential to investigate biologically relevant problems, such as changes in the material properties of supported lipid bilayers, cells, or other biological surfaces under the influence of external factors. The chapter thoroughly describes: (1) the theoretical basis for the SPAM technique, (2) the relationship tapping forces and sample material properties (Young's modulus and the Hamaker constant), (3) how imaging parameters (such as set-point, spring constant, etc.) affect tapping forces, and (4) the application of the SPAM technique to a variety of systems.

\subsection{Introduction}

AFM-based techniques that simultaneously map topography and material properties of surfaces are highly desirable, and a variety of methods have been developed toward accomplish that goal. A straight-forward way to gain insight into sample mechanics is to obtain the time-resolved force interaction between the sample surface and the cantilever tip during standard TMAFM imaging. While controlling the force between the tip and sample during TMAFM experiments is important for imaging soft materials and optimizing resolution, understanding the time-resolved tapping forces can provide insight into material properties, such as adhesion and Young's modulus, of a sample. In 2002, the first successful reconstruction of time-resolved tip/sample forces in a TMAFM

\footnotetext{
${ }^{1}$ This chapter is based on the work, "Recovering Time-Resolved Imaging Forces in Solution by Scanning Probe Acceleration Microscopy: Theory and Application" in Surface Science Tools for Nanomaterials Characterization.
} 
experiment was accomplished by taking the inverse Fourier transform of the Fouriertransformed cantilever trajectory divided by its transfer function [1]. Since then, a variety of other techniques have been developed that can obtain time-resolved tip/sample forces and construct images based on these forces [2-9].

There are several experimental advantages to utilizing tapping forces for mapping the material properties of surfaces. Due to their basis in the TMAFM, such methods offer high spatial resolution and are relatively nondestructive. Other traditional AFM techniques used to measure and/or map material properties of surfaces (i.e., force volume imaging and nanoindentation) often require large deformation of soft samples, which leads to decreased spatial resolution and potentially causes irreversible sample damage. On the contrary, tapping mode forces are adaptive to changes in surfaces properties. Additionally, as scan rates used for simple tapping mode AFM imaging are typically much faster than other methods, the mapping of surface properties can be accomplished in a more timely manner. Thus, TMAFM-based methods provide the ability to probe dynamic changes in a surface's mechanical properties in response to some external stimulus or environmental change.

A TMAFM based method to recover the time-resolved tip/sample force while operating in aqueous solution is scanning probe acceleration microscopy (SPAM, Fig. 1) [4]. The theoretical basis behind SPAM is that during TMAFM experiments, the cantilever acts as an accelerometer, which can be used to extract tip/sample forces by calculating the second derivative of the cantilever deflection signal. Deflection signals from TMAFM experiments contain noise that is amplified by this analysis. As the signal to noise ratio in most commercially available AFMs would overcome the ability to reconstruct the tapping force by this simple method, a means to filter these signals is crucial for SPAM to be useful experimentally. This problem is solved by taking advantage of the fact that information on the tapping event is contained within the higher harmonics which can be obtained from the cantilever deflection trajectory, making it possible to filter the signal by "comb" filtering $[1,10,11]$. Comb filtering is performed by taking the Fourier transform of the noisy deflection trajectory and inverting it, while selectively retaining only the intensities at integer harmonic frequencies of the driving frequency. The effectiveness of comb filtering to reconstruct the deflection signal is 
(a) image lipid bilayer patch on mica and acquire tip deflection trajectory

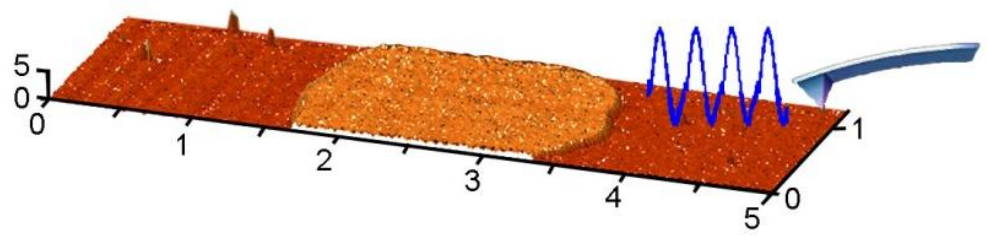

(b)

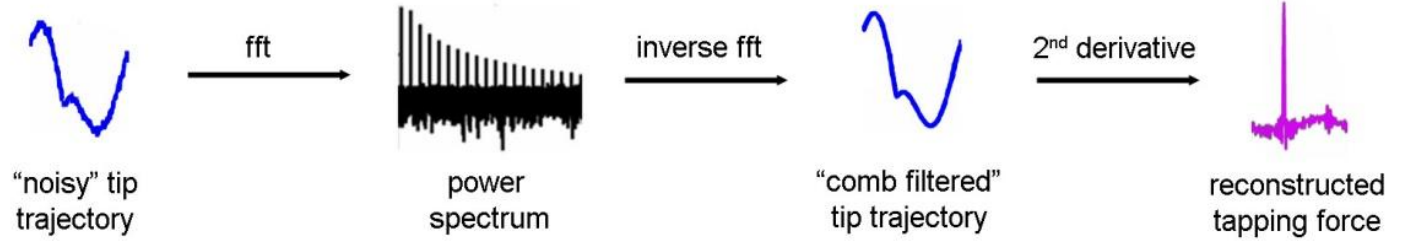

(c) construct maximum tapping force image and correlate tapping force with surface properties
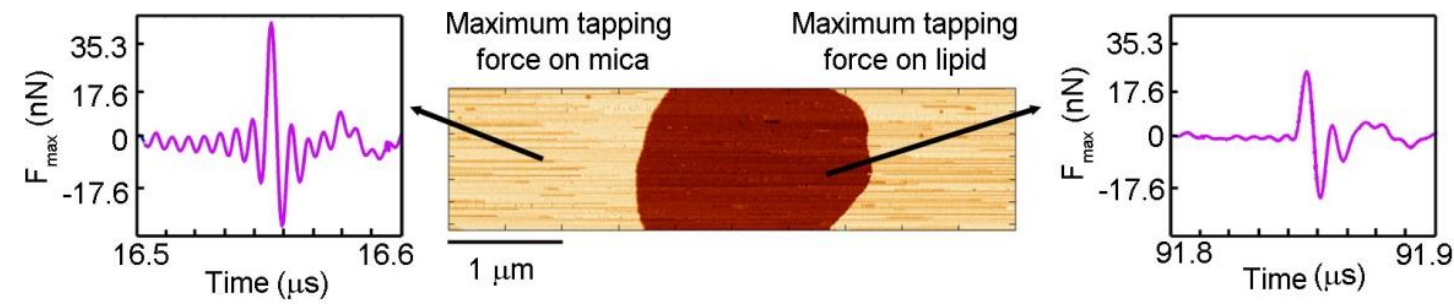

Figure 1. SPAM is a method for reconstructing the time-resolved tip/sample forces associated with TMAFM imaging in solution. In this example, (a) a mica substrate with small supported lipid bilayer patch is imaged to acquire the surface topography while the entire cantilever deflection signal is captured. (b) The deflection trajectory of the cantilever is filtered using a "comb-filter" based on Fourier transforms, and the second derivative of the trajectory is used to reconstruct the tip/sample forces. (c) Images are constructed based on specific features of the tip/sample force, such as the maximum tapping force, which can be related to surface mechanical properties.

dependent on the number of harmonics that appear above the noise level in the Fourier transform. Comb filtering is particularly useful for TMAFM operation in fluid due to the pronounced anharmonic distortion in the deflection signal, which results in a large number of harmonics required for accurate comb filtering [12-14]. The SPAM technique has been validated via numerical simulations and it has proven to be particularly wellsuited for in situ TMAFM experiments on a variety of systems [4, 15-17].

Herein, we describe how time-resolved tip/sample forces are recovered during SPAM operation, present numerical simulations that demonstrate how imaging forces depend on experimental parameters and surface properties, and conclude with examples of SPAM applied to a variety of biological systems. As experimental application of SPAM has been limited to imaging in solution, this chapter focuses on the underlying 
physics of fluid-based TMAFM operation. Although this chapter focuses on SPAM as a method of force reconstruction, much of the presented information is applicable to a broad range of techniques that recover or measure the tip/sample interaction forces while imaging in the tapping mode.

\subsection{Methodology}

\subsubsection{Numerical model of the entire imaging process of TMAFM in solution}

To gain a more complete understanding of the tip/sample interaction associated with TMAFM experiments in liquid, simulations of the TMAFM process were performed using a model developed in MATLAB with SIMULINK (Math Works Inc. Natick, MA; Fig. 2).

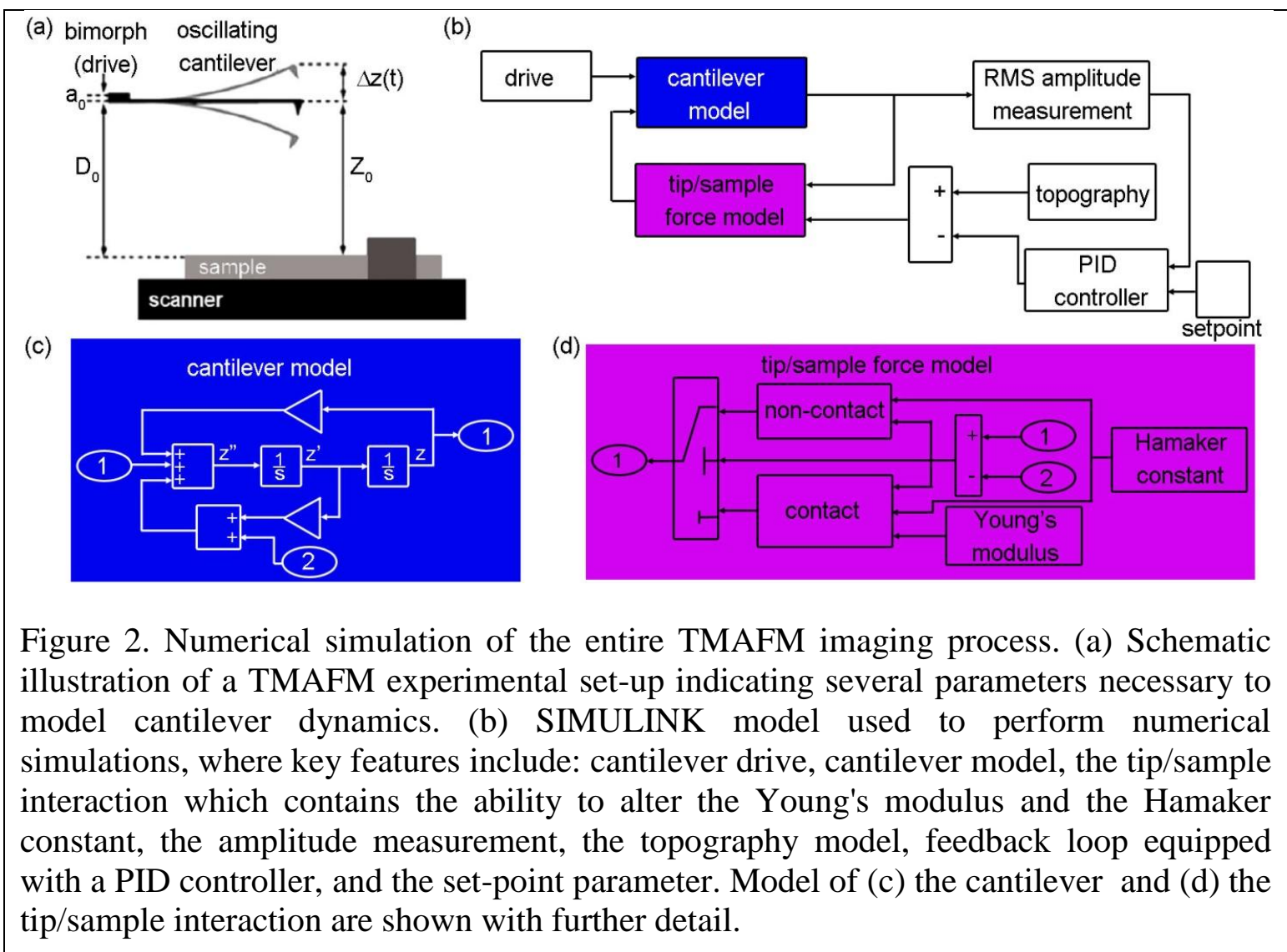


In these simulations, the cantilever was modeled as a as a single degree of freedom, driven, damped harmonic oscillator [18-22]:

$$
m_{e f f} \ddot{z}+b \dot{z}+k\left[z-D_{o}+a_{o} \sin (\omega t)\right]=F_{\text {ext }}
$$

where $m_{e f f}$ is the effective mass of a cantilever, $b$ is the damping coefficient, $k$ is the cantilever spring constant, $z$ is the position of the cantilever with respect to the surface, $D_{0}$ is the resting position of the cantilever base, $a_{0}$ is the drive oscillation amplitude, $\omega$ is the drive frequency, $t$ is the time, and $F_{\text {ext }}$ is the tip/sample force. Although cantilever position, $z$, is often monitored in AFM simulations, in real experiments, most microscopes monitor the amplitude of the cantilever deflection, $y$, which is simply related to the position by the equation

$$
y=z-D_{o}+a_{o} \sin (\omega t)
$$

The quality factor $(Q)$ of the cantilever is the major difference between TMAFM experiments in air and liquid due to the hydrodynamic damping associated with the oscillating cantilever during fluid TMAFM operation [13, 23]. $Q$ can be practically described by:

$$
Q=A_{o} / a_{o}
$$

where $A_{o}$ is the free cantilever oscillation amplitude. In air, $Q$ typically ranges from $200-$ 400 , whereas in fluids $Q$ is only $\sim 1-5$. While operating in air, the free cantilever oscillation amplitude is significantly larger than the drive oscillation amplitude. Consequently, in high $Q$ systems, the cantilever position and deflection trajectories are nearly identical during tapping events except for an offset due to the differing frames of reference (Fig. 3a). However, when operating TMAFM in liquid, i.e. a low $Q$ system, the cantilever deflection and position trajectories differ significantly during tapping events, where the drive amplitude is comparable to the free amplitude. As a result, an 
anharmonic distortion is produced in the deflection trajectory when the cantilever tip contacts the sample surface (Fig. 3b). Therefore, to model TMAFM imaging conditions in solution, it is necessary to rewrite the cantilever motion in terms of deflection, $y$, as

$$
m_{e f f}\left[\ddot{y}-a_{o} \omega^{2} \sin (\omega t)\right]+b\left[y+a_{o} \omega \cos (\omega t)\right]+k y=F_{\text {ext }} .
$$

As an important note, the second mode of the cantilever deflection is not incorporated into this model, but it can play a significant role in cantilever motion near surfaces in fluid [24]; however, contributions from the second mode are often negligible when imaging soft biological samples in solution [25]. In any case, higher eigenmodes of the cantilever can complicate analysis of higher harmonic signals. Additionally, in experimental systems, there are several sources of distortion in cantilever deflection signals. These include nonlinearities of the detector and electronics of the AFM that should be taken into account when comparing numerical simulations to experimental results.

During TMAFM operation, the separation distance between the cantilever tip and the sample surface is constantly change due to the oscillatory behavior of the cantilever. This motion allows the tip to intermittently contact the sample during each oscillation cycle. Consequently, there are two tip/sample interaction regimes that are required for modeling the time-resolved imaging forces: (1) when the cantilever tip and sample surface are not in contact and (2) when the tip and

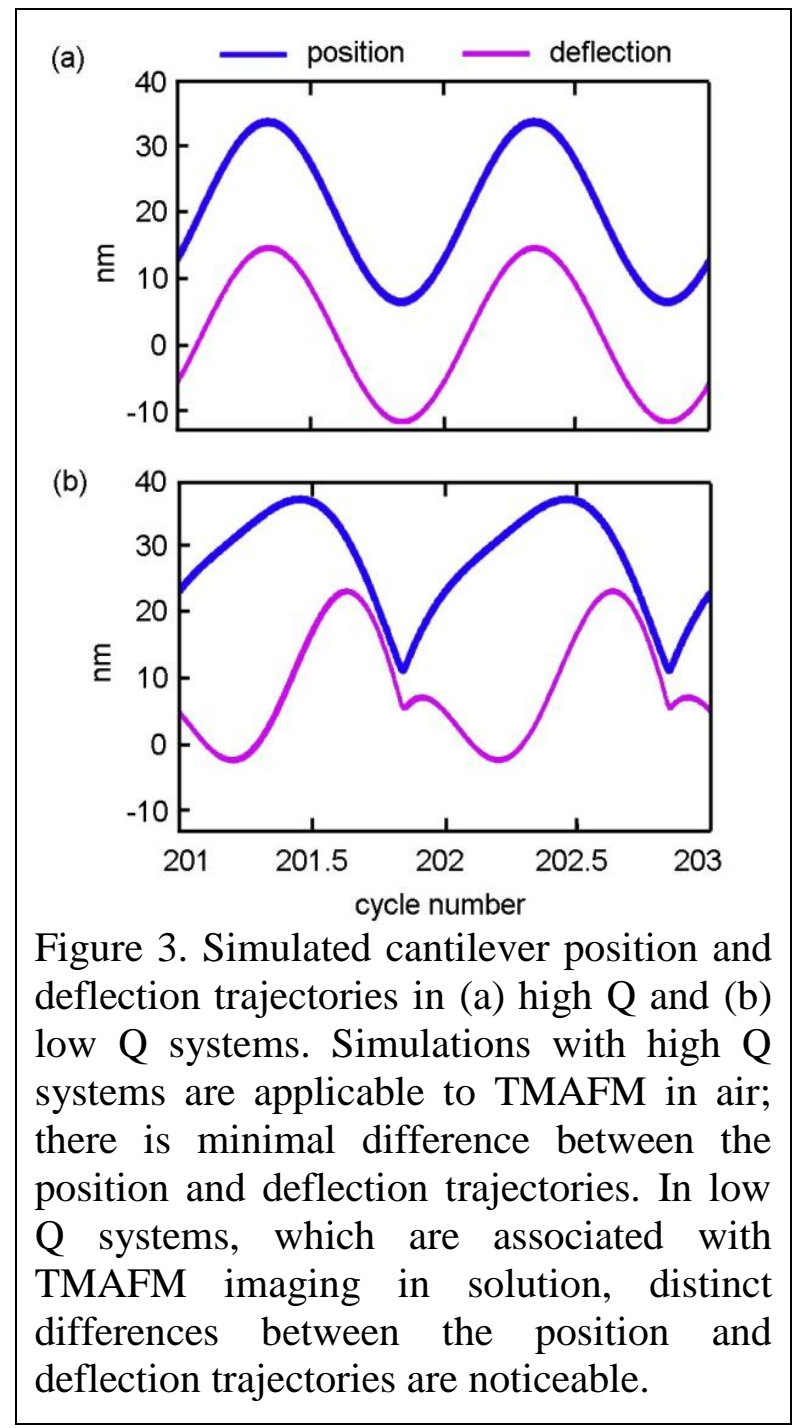


surface make contact during the tapping event. In scenario (1), the separation distance between the cantilever tip and the sample surface, $z$, is greater than the interatomic distance, $a_{D M T}$. When the cantilever tip contacts the surface in situation (2), $z$ is equal to or smaller than $a_{D M T}$. For scenario (1) the force is described by the Derjaguin-LandauVerway-Overbeek (DLVO) theory [26]. However, because most biological experiments are performed in a saline buffer under near physiological conditions, the forces associated with the electric double layer effect are negligible, which results in a relatively short Debye length [25]. As experiments described later were performed under a high salt condition (phosphate buffered saline), the external force can be approximate and simplified using the van der Waals interaction between a sphere (the cantilever tip) and a flat surface [26]:

$$
F_{\text {ext }}=-\frac{H R_{\text {tip }}}{6 z^{2}} \text { for } z>a_{D M T}
$$

where $H$ is the Hamaker constant and $R_{\text {tip }}$ is the tip radius [27]. During the second interaction regime where the cantilever tip contacts the sample surface, the force interaction between the tip and the surface can be described using a DMT potential (Derjaguin-Muller-Toporov):

$$
F_{\text {ext }}=\frac{4}{3 \kappa_{\text {eff }}} \sqrt{R_{\text {tip }}}\left(a_{D M T}-z\right)^{3 / 2}-\frac{H R_{t i p}}{6 a_{D M T}^{2}} \text { for } z \leq a_{D M T}
$$

where

$$
\kappa_{\text {eff }}=\frac{1-v_{t i p}^{2}}{\pi E_{\text {tip }}}+\frac{1-v_{\text {sample }}^{2}}{\pi E_{\text {sample }}}
$$

where $E_{t i p}, v_{t i p}$ and $E_{\text {sample }}, v_{\text {sample }}$ are the Young's modulus and is the Poisson coefficient of the tip or sample, respectively.

Additionally, a feedback loop equipped with an integral and proportional gains was incorporated into the numerical model to simulate the entire TMAFM imaging process (Fig. 2). Implementation of a feedback loop was accomplished by systematically determining the cantilever amplitude for each oscillation cycle, making a direct 
comparison of this amplitude to a predetermined set-point amplitude, and continuously adjusting the tip/sample separation distance to maintain the set-point amplitude. In this way, a simulated trace of the surface, which is simply the required correction in tip/sample separation distance, was obtained for different model surface topographies. Combined with the ability to change the values of the $H$ and/or surface $E$, simulations of imaging a wide array of surfaces with TMAFM were performed to gain a more complete understanding of how the time-resolved tip/sample forces responds to surface properties.

To illustrate the validity of the numerical model, TMAFM simulations in a low $\mathrm{Q}$ system were compared to AFM experiments performed in solution (Fig. 4). For these numerical simulations, the model surface contained a hard region (with a Young's modulus, $E$, of $60 \mathrm{GPa}$ and Hamaker constant, $H$, of $3.5 \mathrm{aJ}$ ) that surrounded a $5 \mathrm{~nm}$ tall softer region (with $E=20 \mathrm{GPa}$ and $H=0.35 \mathrm{aJ}$ ). These simulation parameters were chosen to mimic a typical TMAFM experiment on a supported lipid bilayer patch $(5 \mathrm{~nm}$ is the approximate height of an uncompressed supported lipid bilayer) on a mica surface so that comparisons could be made between simulations and experiments. The timeresolved tip/sample forces from experiments were obtained using SPAM. The simulated
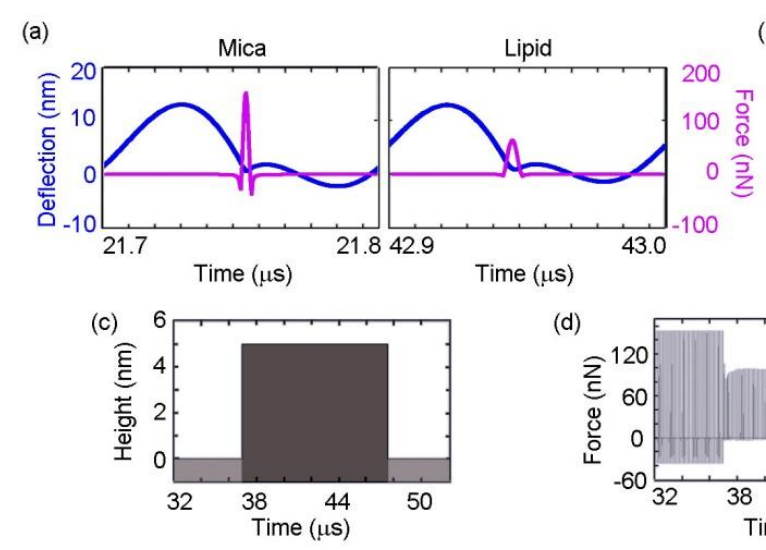

(b)

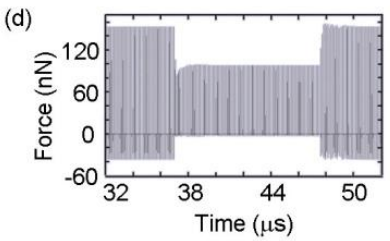

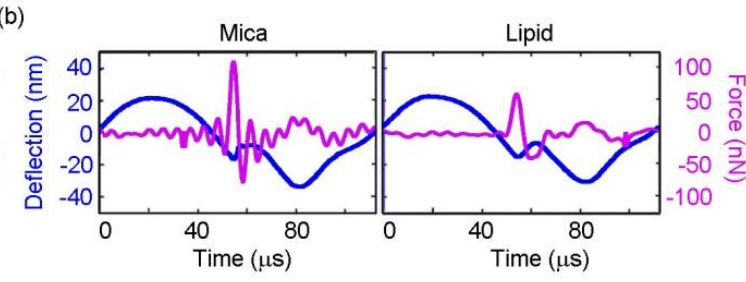

(e)

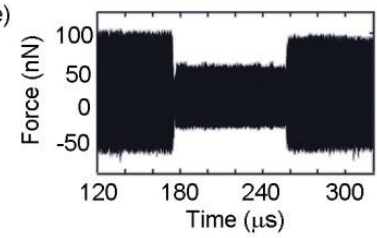

Figure 4. Comparison of simulated (a) and experimental (b) cantilever deflection and force trajectories. (a) Simulations of the cantilever deflection trajectory (blue) and the force trajectory (purple) on hard $(E=60 \mathrm{GPa}, H=3.5 \mathrm{aJ})$ or soft $(E=20 \mathrm{GPa}, H=0.35$ aJ) surfaces, respectively. (b) Experimental deflection and force trajectories of a mica surface and a supported lipid bilayer patch, respectively. (c) Simulated surface which included a $5 \mathrm{~nm}$ soft step, the location of which is indicated by gray shading. (d) Simulated force trajectory of one scan line across a model surface. (e) An experimental force trajectory for an entire scan line on a mica surface with a supported lipid bilayer patch in the center. 
deflection trajectories captured the characteristic anharmonic distortion in the deflection trajectory that is a hallmark of TMAFM experiments performed in solution [13]. There are two features of the tapping force that are of interest here: (1) the maximum tapping force, $F_{\max }$, defined as the most positive force experienced between the cantilever tip and the sample surface during each cantilever oscillation cycle, and (2) the minimum tapping

force, $F_{\text {min }}$, defined as the most negative force experienced between the cantilever tip and the sample surface. The magnitude of both $F_{\max }$ and $F_{\min }$ responded in an analogous way when imaging the model step in simulation or the supported lipid bilayer in the experiment. This confirmed that the numerical model is capable of capturing qualitative features in both cantilever deflection and tip/sample forces associated with TMAFM. The actual relationship between $F_{\max }, F_{\min }, E$, and $H$ will be explored in greater detail later.

\subsubsection{Implementation of SPAM}

In TMAFM experiments, deflection trajectories contain noise. The noise of the cantilever deflection trajectory can be filtered using a Fourier transform based harmonic comb filter. In this process, a sliding window Fourier transform is performed on the cantilever deflection signal, and only intensities in the resulting power spectrum corresponding to integer harmonic frequencies of the drive are kept. These intensities are then used to reconstruct a deflection signal, $y_{r e c}(t)$, by an inverse Fourier transform based on the equation:

$$
y_{\text {rec }}(t)=\hat{f}^{-1}\left[y(\omega) \sum_{k=1}^{N} \delta\left(\omega-k w_{\text {oper }}\right)\right]
$$

where $\delta$ is Dirac's delta function, $\omega_{\text {oper }}$ is the operating frequency, and $N$ is the highest harmonic distinguishable about the noise level. The effectiveness of the comb filter to accurately reconstruct the deflection trajectory requires a sufficient number of harmonics to be above the noise level in the power spectrum, and an $N$ of 20 is typical. As previously mentioned, the cantilever deflection trajectory of fluid-based TMAFM experiments has a distinct distortion associated with the tapping event $[13,14,23]$. This distortion results in an adequate number of higher harmonics above the noise to facilitate comb filtering and reconstruction of the tip/sample tapping force from the second 
derivative (or acceleration) of the cantilever deflection signal. The sliding window of this filter typically contains 5-10 oscillation cycles, and the window is advanced one oscillation cycle at a time to maintain local information about the tip/sample interaction. Once $y_{\text {rec }}(t)$ is obtained, the second derivative of the cantilever deflection trajectories can be taken to obtain the cantilever acceleration. Cantilever acceleration can then be used to create a spatially resolved force map through multiplication with the effective mass of the cantilever, $m_{\text {eff }}$, to obtain the time-resolved tapping force between the tip and the sample, based on a simple rearrangement of Eq. 4 to:

$$
\ddot{y}=\frac{1}{m_{e f f}}\left[F_{e x t}-b \dot{y}-k y+m_{e f f} \omega^{2} a_{o} \sin (\omega t)-b a_{o} \omega \cos (\omega t)\right] .
$$

This process essentially treats the cantilever as a local accelerometer that can be raster scanned across a surface. In this way, topographic and tip/sample force information can be obtained in one experiment simultaneously, providing the basis for SPAM. The last four terms of Eq. 9 are associated with the drive acting on the cantilever, and as a result, oscillate at the drive frequency. These extraneous terms can be easily distinguished from the tip/sample force, and can even be suppressed from the reconstructed force by excluding the fundamental frequency, i.e. drive frequency, from the inverse Fourier transform. However, when this frequency is excluded, the accuracy of the recovered force is diminished by a reduction in magnitude. Additionally, information contained in the harmonics below the noise level is lost when the comb filter is used to produce the reconstructed trajectory. It is also important to note that the harmonic comb filter should only be applied to periodic motion of the kind commonly observed in TMAFM, and it is not applicable to motion which exhibits period doubling or aperiodic/chaotic character.

\subsection{Key research findings}

\subsubsection{Features of the tip/sample force are independent of surface topography}

TMAFM has been used extensively to image a wide variety of sample surfaces, with topographical features ranging from the subnanometer scale to several microns. For tapping mode imaging forces to be related to material properties of surfaces, features of the time-resolved tip/sample tapping force must be independent of surface topography. 
(a)

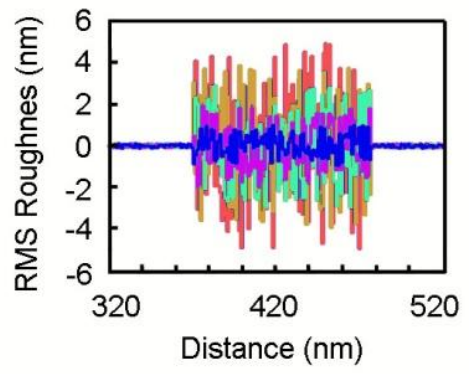

(d)

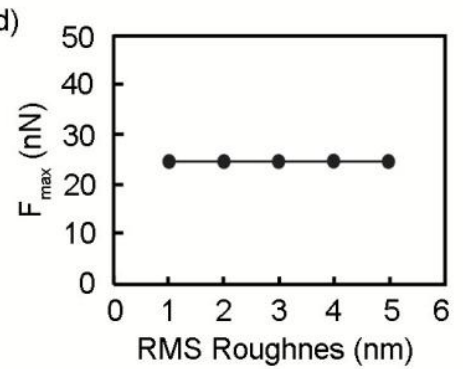

(g)

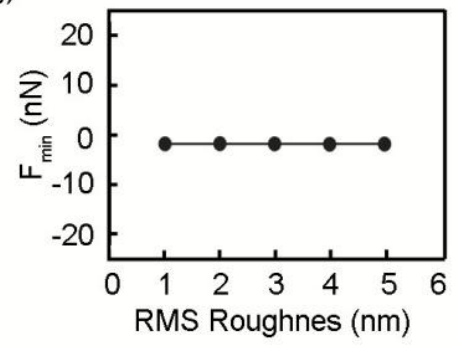

(b)

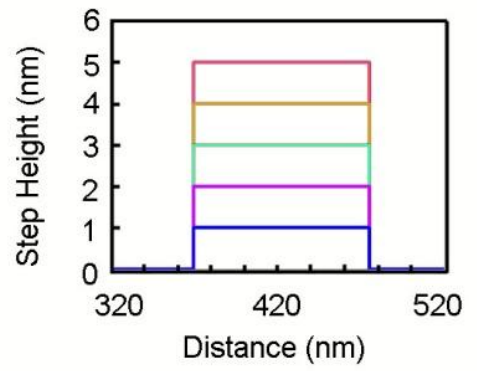

(e)

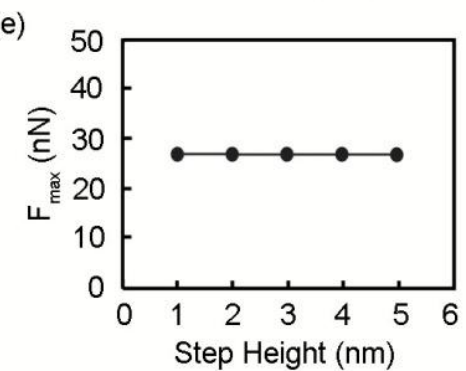

(h)

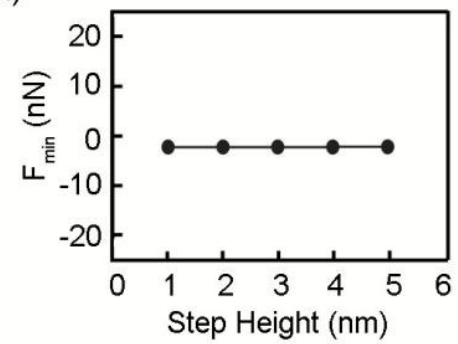

(c)

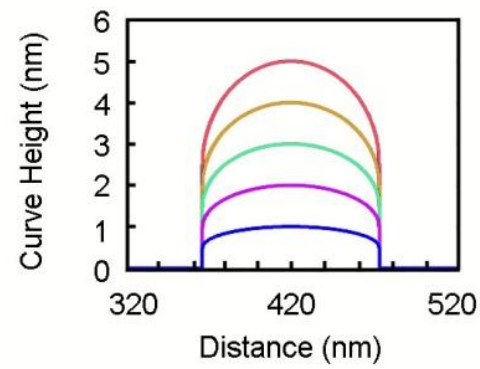

(f)

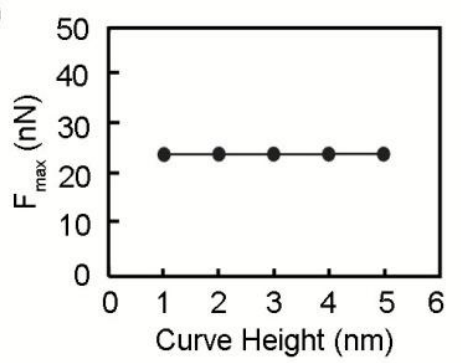

(i)

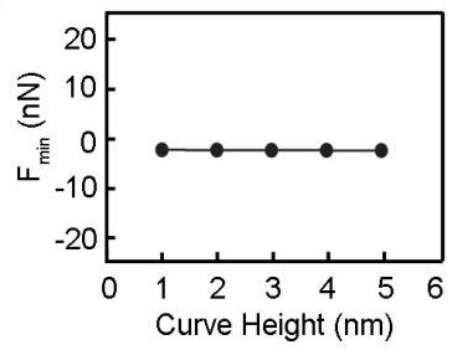

Figure 5. Simulations to determine the effect of surface topography on tip/sample imaging forces. A variety of surface topographies were modeled: (a) a rough surface, (b) a flat step, and (c) a curved feature where roughness or height was varied from 1-5 nm. The maximum and minimum tapping forces, (d-f) and (g-i) respectively, were averaged over the altered surface topography and found to be independent of changes in surface topography.

The role of surface topography on tip/sample tapping forces was determined by running simulations of complete TMAFM experiments. These simulations helped to understand the impact of a variety of model surfaces with distinct morphologies on imaging forces (Fig. 5a-c). Here, three model surfaces are presented: (1) a region with varying RMS roughness, (2) a flat step, and (3) a curved feature. These model surfaces represented potential topographies that may be encountered in real AFM experiments. The size of these features was systematically increased to determine how changes in $F_{\max }$ and $F_{\min }$ were associated with surface topographies. Importantly, other surface properties (such as Young's modulus, Hamaker constant, etc.) and imaging parameters (set-point ratio, free 
amplitude, cantilever spring constant, etc.) were held constant in these simulations. As a result, the average $F_{\max }$ and $F_{\min }$ associated with imaging the various surfaces did not change as a function of the size of the feature in the model, as long as the feedback loop maintained proper tracking of the surface. $F_{\max }$ values for the various topographies were averaged over the altered surface feature (the rough patch, the step, or the round feature) and had an approximate value of $25 \mathrm{nN}$ for each type of surface and each height variation (Fig. 5d-f). The $F_{\min }$ for each surface variation was approximately $-2 \mathrm{nN}$, which was exactly the same as the flat region of the surface. These findings indicate that as long as the feedback loop remains optimized, that changes in the time-resolved tip/sample tapping forces are independent of topographical features.

\subsubsection{Features of the tip/sample force provide insight into surface mechanical properties}

Although $F_{\max }$ and $F_{\min }$ are both independent of surface topography, it is critical to understand the relationship between features of the tapping force and surface mechanical properties to effectively map changes in material properties across a sample. Further simulations were performed to determine how the relative changes in the rigidity of the surface or surface adhesion to the cantilever tip are reflected in the tip/sample force. Changes in the rigidity of the sample were simulated by altering the value of Young's modulus of the sample ( $\left.E_{\text {sample }}\right)$ in Eq. 7. Changes in adhesion were modeled by altering the value of the Hamaker constant in Eqs. 5 and 6. The surface free energy, $\gamma$, (and thus adhesive force) between the tip and sample is related to the Hamaker constant by [26]

$$
\gamma=\frac{H}{24 \pi a_{D M T}} \approx \frac{H}{2.1 \times 10^{-21}}
$$

Here, illustrative simulations were presented in which the Young's modulus and the Hamaker constant were systematically altered on a predetermined area of a model surface to determine the impact of surface rigidity and adhesion on tapping forces. Surface properties of the $5 \mathrm{~nm}$ tall soft step region were modified while holding the properties of the hard region constant. The step was included in the model to determine how changes 
in mechanical properties resulted in compression of the feature due to imaging forces. The Young's modulus, $E$, and Hamaker constant, $H$, of the surface regions surrounding this step were $E=60 \mathrm{GPa}$ and $H=3.0 \mathrm{aJ}$ for all simulations. Other simulation parameters included an amplitude set-point of 0.7 , a free cantilever oscillation amplitude of $15 \mathrm{~nm}$ (and thereby a tapping amplitude of $10.5 \mathrm{~nm}$ ), a drive frequency of $9.5 \mathrm{kHz}$, and a spring constant of $0.5 \mathrm{~N} / \mathrm{m}$. The simulations were set up to mimic a typical TMAFM experiments, with a $2 \mu \mathrm{m}$ scan line at a scan rate of $2 \mathrm{~Hz}$. The Young's modulus on the step region of the model surface was systematically altered from 6 to $100 \mathrm{GPa}$. As the modulus decreased, the model step became more compliant to compression due to the externally applied force from the tip, and the measured height became smaller (Fig. 6a). The AFM probe pushed deeper into the sample, as a result of the increased compliance of the model step, causing the decreased height measurement. Additionally, the tip was in

(a)

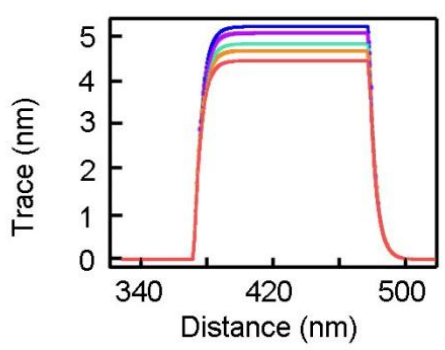

(d)

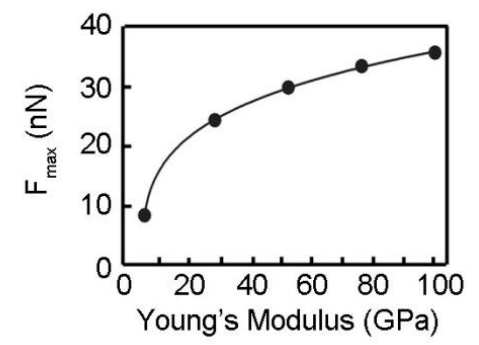

(b)

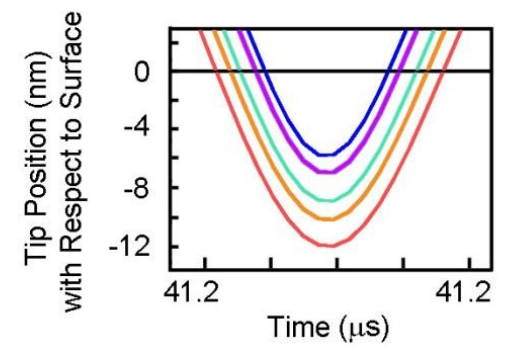

(e)

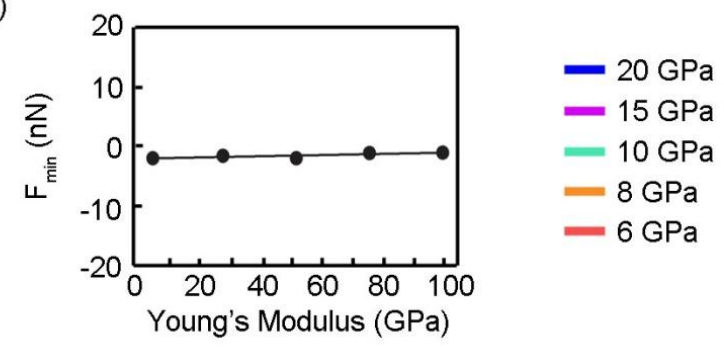

Figure 6. Simulations demonstrate the impact of surface Young's modulus on tip/sample imaging forces. The simulated surface topography included a step, with changing values of $E$, surrounded by a hard surface. (a) The measured topography of the step as a function of $E$ associated with the surface. (b) The position of the cantilever with respect to the sample surface corresponding to an individual tapping event. (c) The tip/sample force corresponding to the tapping event. (d) The average value of the maximum tapping force, $F_{\max }$, plotted as a function of $E$. (e) The average value of the minimum tapping force, $F_{\text {min }}$, plotted as a function of $E$. 
contact with the surface for a longer time during each oscillation cycle (Fig. 6b). The way in which the force was distributed changed in response to increased compliance that was associated with smaller values of surface Young's modulus (Fig. 6c). This is a result of the TMAFM procedure, where imaging is accomplished by maintaining the set-point amplitude, and thus the total force per cantilever oscillation cycle. Specifically, the force was spread out over the longer contact time on more compliant surfaces, which resulted in a reduced $F_{\max }$ that was associated with softer surfaces. Consequently, the magnitude of the $F_{\max }$ was altered with larger values of Young's modulus, and $F_{\min }$ was unresponsive to those changes (Fig. 6d). Additionally, it was found that the relationship between $F_{\max }$ and Young's modulus could be described by a power law. These results suggested that $F_{\max }$ can be used to indicate the relative rigidity of surfaces; however, as demonstrated, this notion is complicated by changes in adhesion between the tip and surface.

Next, the importance of the Hamaker constant the time-resolved tip/sample force was explored by systematically changing the value of surface free energy (and thus adhesion) between the tip and surface (based on Eq. 10). The values of $H$ ranged from 0.5-2 aJ on the step region of the model surface, while maintaining a constant value of Young's modulus (5 GPa), and all other parameters were held constant (Fig. 7).The simulated measured height of the step decreased as the Hamaker constant increased (Fig. 7a), which was a result of a larger magnitude (more negative) tip/sample attractive force between the cantilever tip and sample surface. This attractive tip/sample interaction caused the tip to be pulled deeper into the soft model surface (Fig. 7b); however, when model included a more rigid surface, this effect was minimized. Unlike the simulations with varying Young's modulus, when the tip is pulled deeper into soft surfaces while increasing the value of the Hamaker constant, the contact time between the cantilever tip and the sample surface remains relatively unchanged (Fig. 7b). When the value of the Hamaker constant is increased, a the tip/sample interaction experiences forces with a larger magnitude, i.e. more negative or attractive forces between the tip and surface (Fig. 7c); however, for successful tracking of surface topography, it is required that the total force per oscillation cycle is maintained by the feedback loop. Accordingly, $F_{\max }$ increases in magnitude with larger values of the Hamaker constant to compensate for the 


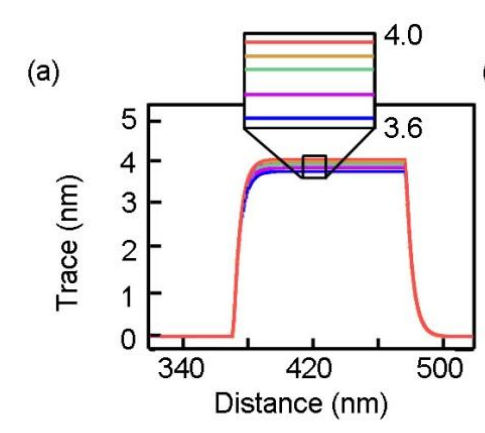

(d)

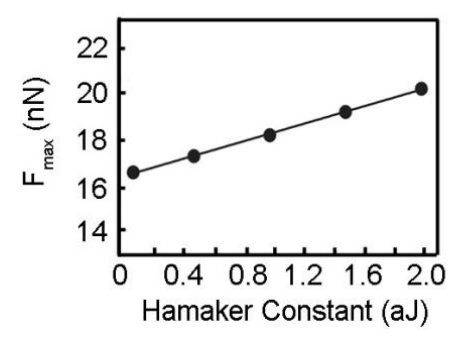

(b)

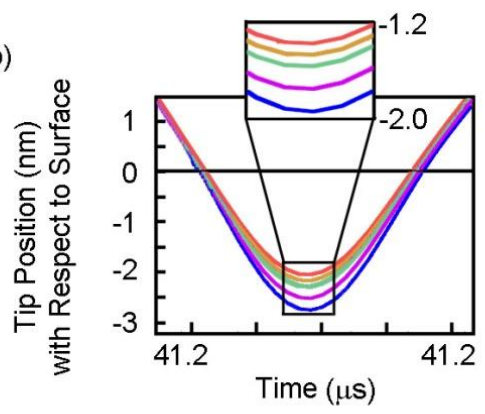

(e)

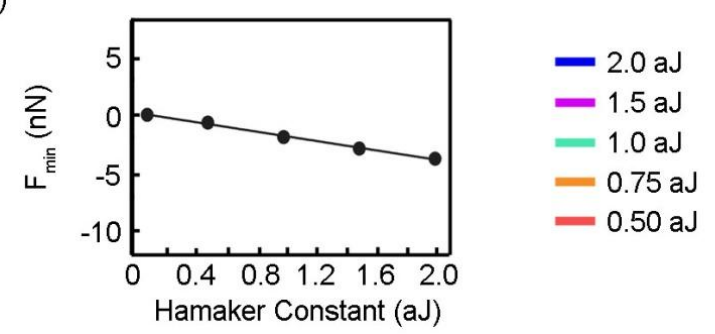

Figure 7. Simulations demonstrating the impact of altering the Hamaker constant on tip/sample imaging forces. The model surface topography includes a step, with changing values of $H$. (a) The measured topography of the step as a function of $H$ associated with the surface. (b) The position of the cantilever with respect to the sample surface corresponding to the tapping event. (c) The tip/sample force corresponding to the tapping event. (d) The average value of the maximum tapping force, $F_{\max }$, plotted as a function of Hamaker constant. (e) The average value of the minimum tapping force, $F_{\min }$, plotted as a function of Hamaker constant.

increased attractive force associated with each tapping event (Fig. 7c). The larger magnitude of $F_{\max }$ due to this increased Hamaker constant can be directly contributed to the deeper penetration of the tip into the surface, which enlarged the force associated with the Hertzian term in Eq. 6. Consequently, the magnitude of both $F_{\max }$ and $F_{\min }$ responds linearly with changes in the Hamaker constant (Fig. 7d-e).

Taken together, these simulations on model surfaces demonstrated that specific features of the time-resolved tip/sample force can be used to map relative changes in the mechanical properties of sample surfaces. Specifically, $F_{\min }$ changed in a linear fashion to increased adhesive forces experienced between the cantilever tip and the sample surface, as simulated by increasing the value of the Hamaker constant. This demonstrated that changes in $F_{\text {min }}$ are independent of the Young's modulus, and that $F_{\text {min }}$ images can be used to measure the adhesion between a cantilever tip and a sample surface. Understanding relative changes in Young's modulus is more complicated because $F_{\max }$ is 
dependent on both the Hamaker constant (linearly) and Young's modulus (power law). However, the contribution to $F_{\max }$ due to changes in the Hamaker constant can be estimated from $F_{\text {min }}$, which can be used to determine if observed changes in $F_{\max }$ are at least partially derived from variations in the Young's modulus of the surface.

\subsubsection{The impact of imaging parameters on the tip/sample force}

Simulations have demonstrated that $F_{\max }$ and $F_{\min }$ change predictably to reflect surface mechanical properties; however, it is necessary to understand how imaging parameters such as spring constant, free cantilever oscillation amplitude, and set-point ratio impact the time-resolved tip/sample forces to make accurate comparisons across samples. Imaging parameters can be related to the total tip/sample force per oscillation cycle by the equation [28]:

$$
F_{\text {total }} \approx 0.5 k a_{o} \frac{\Delta A}{A_{o}}
$$

where $\Delta A$ is the difference between the free amplitude (or amplitude when the tip is oscillating freely above the surface) and the tapping amplitude, $A_{o}-A$. The relationship between imaging parameters and tip/sample force has been explored by simulations and experimentally [15, 17, 29]. Based on Eq. 11, as the drive amplitude, and thereby the free amplitude, is increased for any given cantilever, the total imaging force per oscillation cycle should increase systematically if the set-point is maintained. This concept was validated through

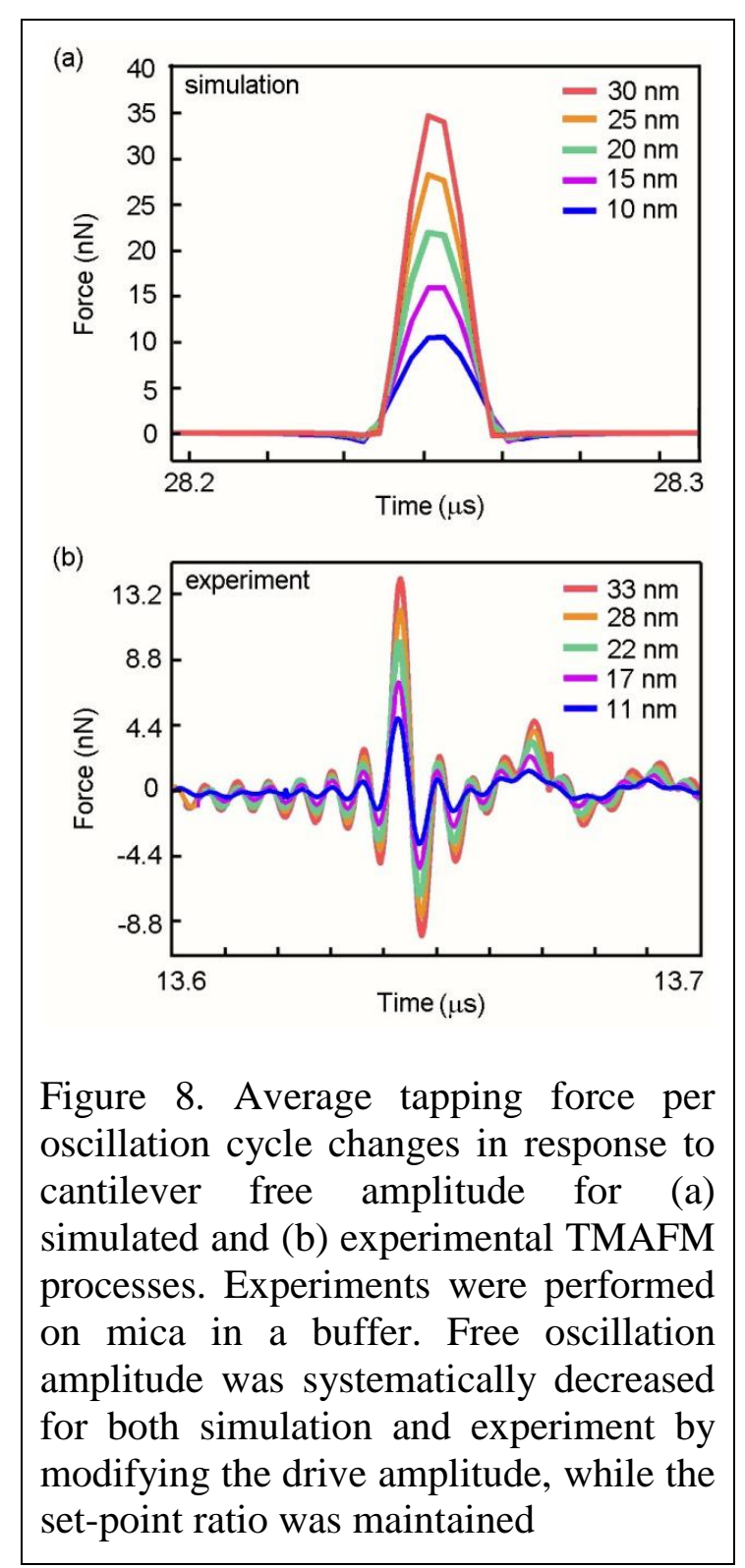


simulation and experiment (Fig. 8). In the simulation and experiment, as the drive amplitude was increased, a larger free amplitude was experienced, and the set-point ratio was maintained. Additionally, all other imaging parameters were held constant for both simulation and experiment. Under such conditions, the contact time between the tip and surface was independent of free amplitude; therefore, the observed increase in $F_{\max }$ resulted from a larger total force per oscillation cycle associated with increasing free amplitude.

For any given free amplitude, decreasing the set-point ratio resulted in an increase in the total force per oscillation cycle. Simulations and experiments have also been performed to demonstrate the effect of $A_{s p}$ on tapping forces $[16,30]$. If only the value of $A_{s p}$ was modified while all other imaging parameters were maintained, the total force per oscillation cycle and $F_{\max }$ increased with a power law dependence as $A_{s p}$ was reduced for both simulation and experiment (Fig. 9). Consistent with previously described simulations, the tip/sample forces were dependent on the Young's modulus of the surface. This demonstrated that the force applied to the sample can be controlled by adjusting imaging parameters; however, the relative relationships between mechanical surface properties and specific features of the time-resolved tip/sample force are still valid, provided that imaging parameters are maintained throughout the analysis.

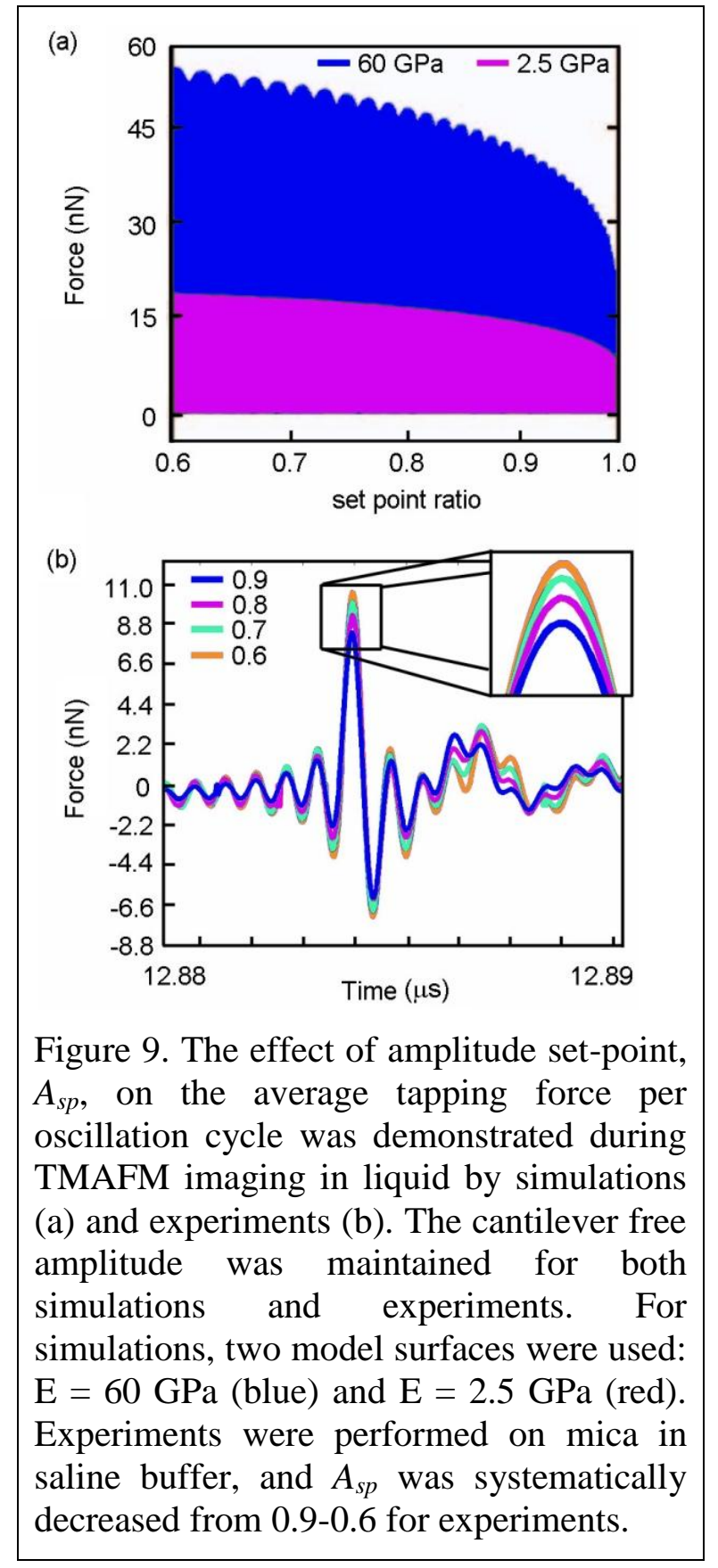


Numerical simulations were also performed to determine the impact the cantilever spring constant on imaging forces, specifically $F_{\max }$ and $F_{\min }$. It was necessary to maintain other imaging parameters (such as the set-point ratio, the drive amplitude, the free amplitude, the resonance frequency, and the drive frequency) to facilitate comparisons between differing spring constants. For the first set of simulations, the value of the Hamaker constant was maintained while the value of Young's modulus was systematically modified. Raising the spring constant from $0.1-1.0 \mathrm{~N} / \mathrm{m}$ resulted in a shift in the magnitude of $F_{\max }$ for any given surface Young's modulus. However, the power law dependence of $F_{\max }$ on Young's modulus was maintained for any given cantilever

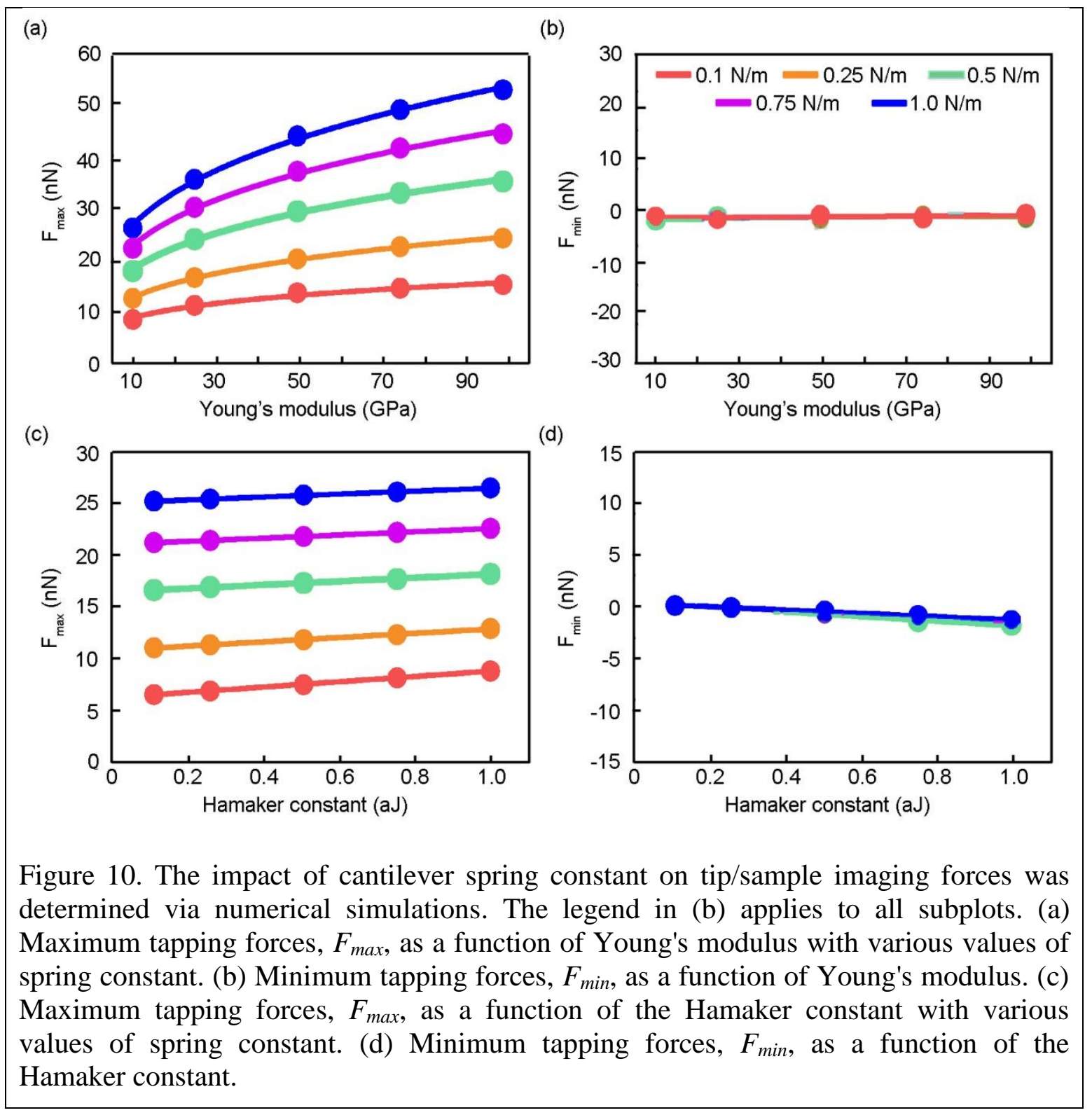


spring constant (Fig. 10a), and $F_{\min }$ was unaffected by modification of the spring constant (Fig. 10b). A similar trend was observed when simulations were performed to determine how increasing the cantilever spring constant altered imaging forces related to changes in the Hamaker constant: as the spring constant increased, $F_{\max }$ increased for any given Hamaker constant, with no change in $F_{\min }$ (Fig. 10c). However, for any given spring constant, as the Hamaker constant was systematically increased from 0.1-1.0 aJ, the linear relationships between both $F_{\max }$ and $F_{\min }$ were maintained. Therefore, as long as a single cantilever is used, features of tapping forces can be directly compared to determine relative changes in sample mechanical properties.

\subsubsection{Experimental applications of SPAM}

The first successful application of the SPAM technique on an experimental system utilized supported lipid bilayer patches on mica (Fig. 11) [31]. Supported bilayers have a significantly lower elastic modulus than mica; therefore, this system provides a surface with a soft and hard regions (supported bilayer and mica, respectively). Histograms of

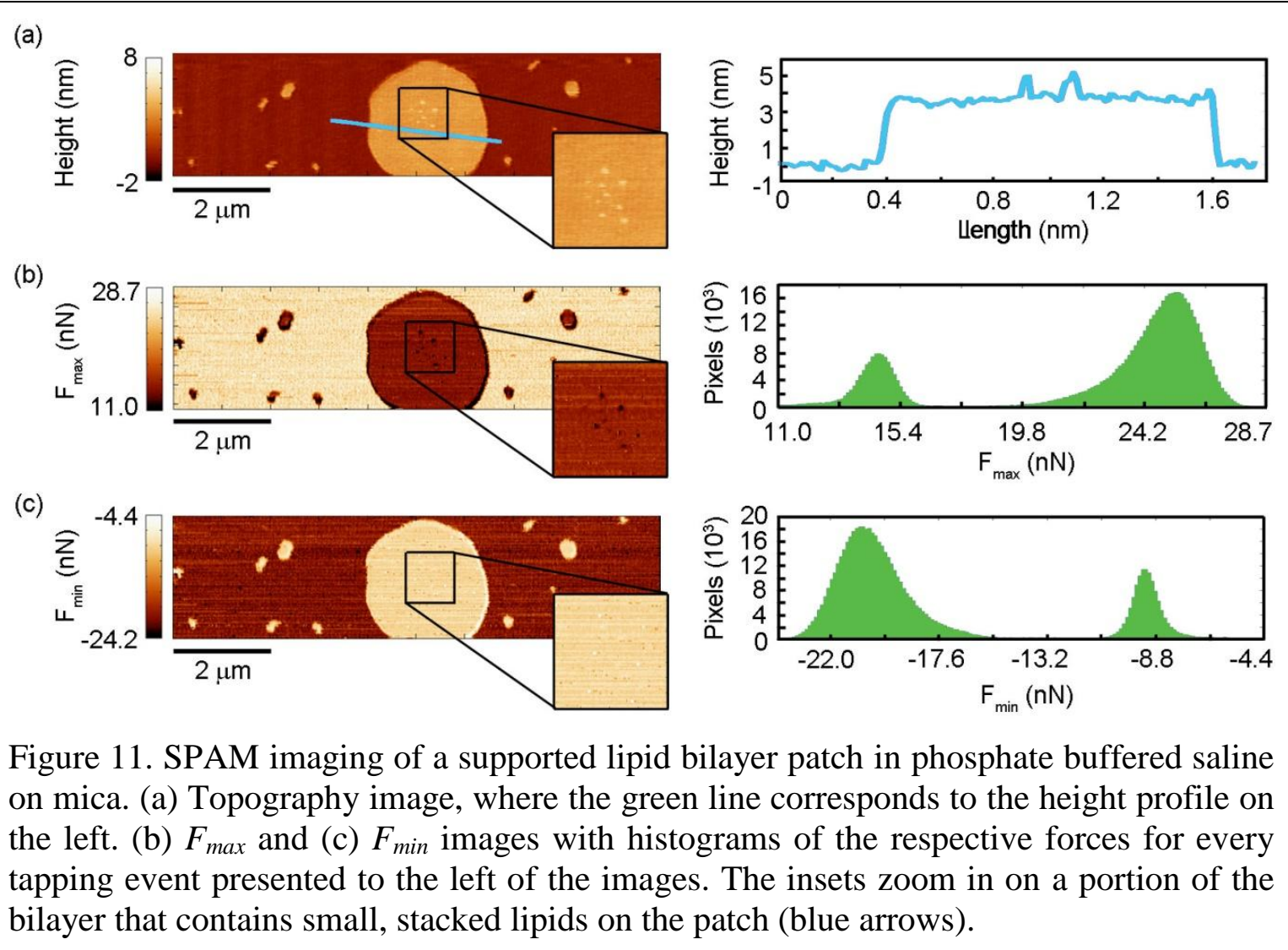


$F_{\max }$ and $F_{\min }$ associated with imaging this system are distinctly bimodal. Each mode corresponds to either the mica or bilayer portions of surface, and provides excellent contrast in the reconstructed force images (Fig. 11b-c). In agreement with the simulation results, the values of $F_{\max }$ corresponding to the bilayer were lower in comparison to those corresponding to mica. The magnitude of $F_{\min }$ associated with imaging mica was larger than that for the bilayer patch, indicating that the tip adhered more strongly to the mica surface. Interestingly, there were small regions on the supported bilayer patch, approximately $~ 30-150 \mathrm{~nm}$ wide, that appeared to be a second bilayer stacked on top of the first (Fig. 11, inset). The appearance of these regions was associated with a decrease in $F_{\max }$, with no discernible change in $F_{\min }$. Those changes suggested that the stacked bilayers had a reduced Young's modulus without a corresponding increase in adhesion.

Several studies have measured mechanical changes in supported lipid membranes using SPAM. For example, SPAM has been used to determine the effect of cholesterol on the modulus of lipid bilayers [17], how exposure to lipoprotein particles alters membrane mechanics [32], how membrane disruption induced by a variety amyloid-forming proteins alter local mechanical properties of lipid bilayers [15, 33-35], and how mechanical changes associated with membrane curvature enhances the binding and aggregation of the huntingtin peptide [36].

While SPAM has mainly been applied to studies involving supported lipid membranes, the technique can be applied to a variety of systems. For example, SPAM
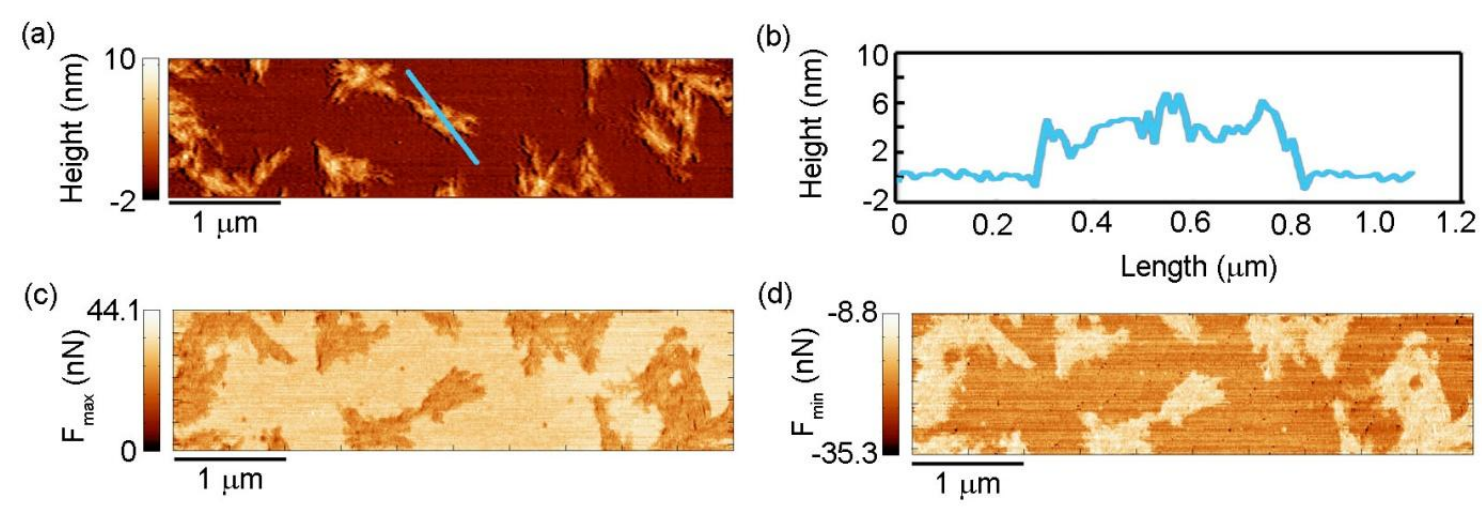

Figure 12. Images of aggregates of a polyglutamine peptide deposited on a mica substrate created from the SPAM procedure. (a) Topography image where the green line corresponds to the (b) height profile. Excellent contrast is observed in both the (c) $F_{\max }$ and (d) $F_{\min }$ images between the proteinaceous material and the mica substrate. 
has been used to verify the magnitude of tapping forces associated with imaging fragile viral capsids and nanoparticles [25, 36]. Contrast associated with imaging forces is also achievable between protein aggregates and an underlying mica substrate (Fig. 12). Contrast in $F_{\max }$ and $F_{\min }$ was also observed when using SPAM on a variety of materials imaged in solution, such as a film consisting of a blend of polystyrene (PS, elastic modulus of $\sim 2 \mathrm{GPa}$ ) and polyolefin elastomer (LDPE, elastic modulus of $\sim 0.1 \mathrm{GPa}$ ) supported on a silicon substrate (Fig. 13).

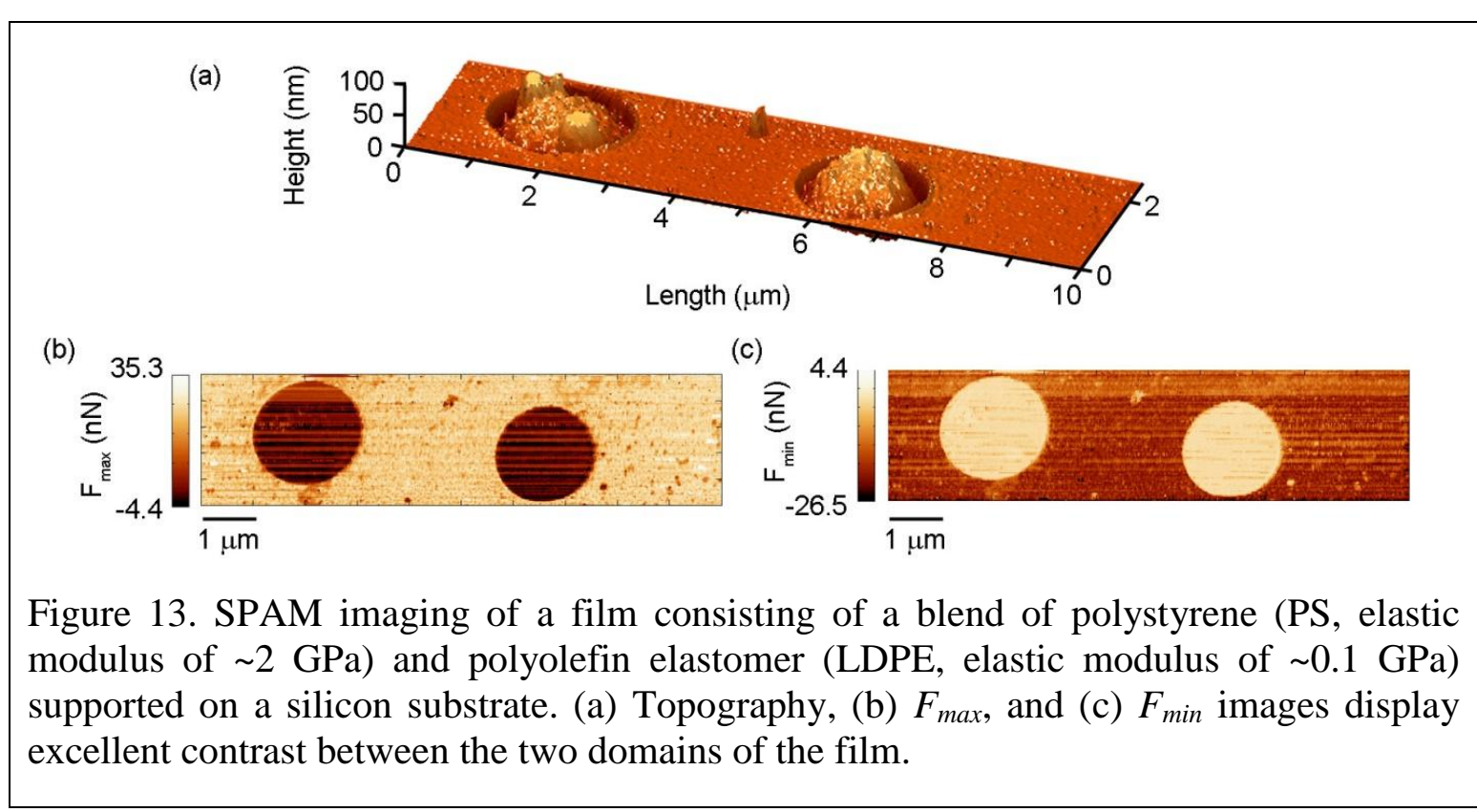

\subsection{Conclusions}

The ability to simultaneously map topography and mechanical properties of surfaces is an attractive feature of proximal probe-based microscopies, and accomplishing this functionality has long been a focus within the field. Although recent advances have made this a reality, where SPAM represents just one such technique, there is still considerable effort required to refine these techniques. This chapter has highlighted the applicability of the SPAM technique for simultaneously imaging and mapping the mechanical properties of surfaces through a combination of simulations and experiments on a variety of samples. The simulations presented here provided insight into the relationships between tapping forces, surface mechanical properties and imaging parameters. These relationships are also hold true for other TMAFM-based force reconstruction techniques. 
Importantly, these studies demonstrated that spatially resolved force maps can be directly correlated to material properties of the surface, such as modulus and adhesion. Additionally, because the SPAM technique is especially suitable for fluid TMAFM experiments, it has significant potential for biological applications. The following two chapters further demonstrate the applicability of the SPAM technique to supported lipid bilayer systems.

\subsection{References}

1. Stark, M., R.W. Stark, W.M. Heckl, and R. Guckenberger, Inverting dynamic force microscopy: From signals to time-resolved interaction forces. Proceedings of the National Academy of Sciences of the United States of America, 2002. 99(13): p. 8473-8478.

2. Balantekin, M., A.G. Onaran, and F.L. Degertekin, Quantitative mechanical characterization of materials at the nanoscale through direct measurement of time-resolved tip-sample interaction forces. Nanotechnology, 2008. 19(8).

3. Garcia, R. and E.T. Herruzo, The emergence of multifrequency force microscopy. Nature Nanotechnology, 2012. 7(4): p. 217-226.

4. Legleiter, J., M. Park, B. Cusick, and T. Kowalewski, Scanning probe acceleration microscopy (SPAM) in fluids: mapping mechanical properties of surfaces at the nanoscale. Proceedings of the National Academy of Sciences of the United States of America, 2006. 103: p. 4813-4818.

5. Sahin, O., S. Magonov, C. Su, C.F. Quate, and O. Solgaard, An atomic force microscope tip designed to measure time-varying nanomechanical forces. Nature Nanotechnology, 2007. 2(8): p. 507-514.

6. Sahin, O., C.F. Quate, O. Solgaard, and A. Atalar, Resonant harmonic response in tapping-mode atomic force microscopy. Physical Review B, 2004. 69(16).

7. Sahin, O., G. Yaralioglu, R. Grow, S.F. Zappe, A. Atalar, C. Quate, and O. Solgaard, High-resolution imaging of elastic properties using harmonic cantilevers. Sensors and Actuators a-Physical, 2004. 114(2-3): p. 183-190. 
8. Sarioglu, A.F., S. Magonov, and O. Solgaard, Tapping-mode force spectroscopy using cantilevers with interferometric high-bandwidth force sensors. Applied Physics Letters, 2012. 100(5).

9. Sarioglu, A.F. and O. Solgaard, Modeling, design, and analysis of interferometric cantilevers for time-resolved force measurements in tapping-mode atomic force microscopy. Journal of Applied Physics, 2011. 109(6).

10. Hillenbrand, R., M. Stark, and R. Guckenberger, Higher-harmonics generation in tapping-mode atomic-force microscopy: Insights into the tip-sample interaction. Applied Physics Letters, 2000. 76(23): p. 3478-3480.

11. Stark, R.W. and W.M. Hecki, Higher harmonics imaging in tapping-mode atomic-force microscopy. Review of Scientific Instruments, 2003. 74(12): p. 5111.

12. Chen, G.Y., R.J. Warmack, P.I. Oden, and T. Thundat, Transient response of tapping scanning force microscopy in liquids. Journal of Vacuum Science \& Technology B, 1996. 14(2): p. 1313-1317.

13. Legleiter, J. and T. Kowalewski, Insights into fluid tapping-mode atomic force microscopy provided by numerical simulations. Appl. Phys. Lett., 2005. 87: p. 163120/1-163120/3.

14. Putman, C.A.J., K.O. Vanderwerf, B.G. Degrooth, N.F. Vanhulst, and J. Greve, Tapping mode atomic force microscopy in liquid. Applied Physics Letters, 1994. 64(18): p. 2454-2456.

15. Burke, K.A., E.A. Yates, and J. Legleiter, Amyloid-Forming Proteins Alter the Local Mechanical Properties of Lipid Membranes. Biochemistry, 2013. 52(5): p. 808-817.

16. Kumar, B., P.M. Pifer, A. Giovengo, and J. Legleiter, The effect of set point ratio and surface Young's modulus on maximum tapping forces in fluid tapping mode atomic force microscopy. J. Appl. Phys., 2010. 107.

17. Shamitko-Klingensmith, N., K.M. Molchanoff, K.A. Burke, G.J. Magnone, and J. Legleiter, Mapping the Mechanical Properties of Cholesterol-Containing Supported Lipid Bilayers with Nanoscale Spatial Resolution. Langmuir, 2012. 28: p. $13411-13422$. 
18. Garcia, R. and R. Perez, Dynamic atomic force microscopy methods. Surface Science Reports, 2002. 47(6-8): p. 197-301.

19. Burnham, N.A., O.P. Behrend, F. Oulevey, G. Gremaud, P.J. Gallo, D. Gourdon, E. Dupas, A.J. Kulik, H.M. Pollock, and G.A.D. Briggs, How does a tip tap? Nanotechnology, 1997. 8(2): p. 67-75.

20. Kuhle, A., A.H. Sorensen, and J. Bohr, Role of attractive forces in tapping tip force microscopy. Journal of Applied Physics, 1997. 81(10): p. 6562-6569.

21. Salapaka, M.V., D.J. Chen, and J.P. Cleveland, Linearity of amplitude and phase in tapping-mode atomic force microscopy. Physical Review B, 2000. 61(2): p. 1106-1115.

22. Song, Y.X. and B. Bhushan, Atomic force microscopy dynamic modes: modeling and applications. Journal of Physics-Condensed Matter, 2008. 20(22).

23. Tamayo, J., Energy dissipation in tapping-mode scanning force microscopy with low quality factors. Applied Physics Letters, 1999. 75(22): p. 3569-3571.

24. Basak, S. and A. Raman, Dynamics of tapping mode atomic force microscopy in liquids: Theory and experiments. Applied Physics Letters, 2007. 91(6): p. 064107-3.

25. Xu, X., C. Carrasco, P. Jose de Pablo, J. Gomez-Herrero, and A. Raman, Unmasking imaging forces on soft biological samples in liquids when using dynamic atomic force microscopy: A case study on viral capsids. Biophysical Journal, 2008. 95(5): p. 2520-2528.

26. Israelachvili, J., Intermolecular \& Surface Forces 1992, London: Academic Press.

27. Derjaguin, B.V., V.M. Muller, and Y.P. Toporov, Effect of contact deformations on the adhesion of particles. Journal of Colloid and Interface Science, 1975. 53(2): p. 314-326.

28. Kowalewski, T. and J. Legleiter, Imaging stability and average tip-sample force in tapping mode atomic force microscopy. J. Appl. Phys., 2006. 99: p. 064903/1064903/5.

29. Guzman, H.V., A.P. Perrino, and R. Garcia, Peak forces in high-resolution imaging of soft matter in liquid. ACS Nano, 2013. 7(4): p. 3198-3204. 
30. Legleiter, J., The effect of drive frequency and set point amplitude on tapping forces in atomic force microscopy: simulation and experiment. Nanotechnology, 2009. 20: p. 245703/1-245703/10.

31. Legleiter, J., M. Park, B. Cusick, and T. Kowalewski, Scanning probe acceleration microscopy (SPAM) in fluids: Mapping mechanical properties of surfaces at the nanoscale. Proceedings of the National Academy of Sciences of the United States of America, 2006. 103(13): p. 4813-4818.

32. Legleiter, J., J.D. Fryer, D.M. Holtzman, and T. Kowalewski, The Modulating Effect of Mechanical Changes in Lipid Bilayers Caused by ApoE-Containing Lipoproteins on A $\beta$ Induced Membrane Disruption. ACS Chem. Neurosci., 2011. 2: p. 588-599.

33. Yates, E.A., S.L. Owens, M.F. Lynch, E.M. Cucco, C.S. Umbaugh, and J. Legleiter, Specific domains of $A \beta$ facilitate aggregation on and association with lipid bilayers. Journal of molecular biology, 2013. 425(11): p. 1915-33.

34. Burke, K.A., K.J. Kauffman, C.S. Umbaugh, S.L. Frey, and J. Legleiter, The interaction of polyglutamine peptides with lipid membranes is regulated by flanking sequences associated with huntingtin. The Journal of biological chemistry, 2013. 288(21): p. 14993-5005.

35. Burke, K.A., K.M. Hensal, C.S. Umbaugh, M. Chaibva, and J. Legleiter, Huntingtin disrupts lipid bilayers in a polyQ-length dependent manner. Biochimica Et Biophysica Acta, 2013. 1828(8): p. 1953-61.

36. Chaibva, M., K.A. Burke, and J. Legleiter, Curvature enhances binding and aggregation of huntingtin at lipid membranes. Biochemistry, 2014. 53(14): p. 2355-2365. 


\subsection{Mapping the mechanical properties of cholesterol-containing supported lipid bilayers with nanoscale spatial resolution ${ }^{1}$}

Many biological processes are influenced by mechanical changes in membranes which are comprised of a variety of lipid components. As a result, the ability to map physicomechanical properties of surfaces with high temporal and spatial resolution is desirable. Tapping mode atomic force microscopy (AFM) has proven to be a useful technique for imaging biological surfaces due to its ability to operate in solution; however, access to information concerning the mechanical properties of these surfaces can also be obtained by reconstructing the time-resolved tip/sample force interactions during the imaging process. Reconstruction of the tip/sample force is achievable by scanning probe acceleration microscopy (SPAM). Herein, the applicability of SPAM to study mechanical properties of supported lipid bilayers with nanoscale spatial resolution via experiment is described. The maximum and minimum tapping forces obtained from the SPAM procedure respond to changes in surface mechanical properties, and these changes can be used to measure mechanical properties of total brain lipid extract bilayers containing various amounts of exogenous cholesterol supported on a mica surface. Furthermore, the appearance of nanoscale raft domains within the bilayer was examined.

\subsection{Introduction}

When working with biological materials, it is of interest to quickly and delicately make measurements to preserve sample integrity, i.e., prevent sample drying or surface damage. Specifically, techniques that quantitatively and temporally monitor, track, and/or characterize mechanical properties of biologically relevant surfaces with nanoscale spatial resolution are desirable [1]. Scanning probe acceleration microscopy (SPAM), which is based on tapping mode atomic force microscopy (TMAFM), is one such technique [2]. As described in Chapter 2, the SPAM technique is especially useful for experiments in solution, thus, it has the potential to investigate biologically relevant problems, such as changes in the material properties of supported lipid bilayers, cells, or other biological surfaces under the influence of external factors.

\footnotetext{
${ }^{1}$ This chapter is based on the article, "Mapping the Mechanical Properties of Cholesterol-Containing Supported Lipid Bilayers with Nanoscale Spatial Resolution" in Langmuir.
} 
Mapping the mechanical properties of supported lipid bilayers is a particularly important application of the time-resolved tip/sample forces because lipid bilayers can be used as models for cellular membranes. Membranes are critical to all living systems, and many biochemical reactions occur on those surfaces. Biological membranes require a high degree of lateral fluidity, while simultaneously maintaining structural integrity. Specifically, biological membranes must be able to perform a variety of functions properly and withstand mechanical and chemical stresses in their physiological environment, and the physical properties of the membrane are key to those processes. Cell membranes are two-dimensional liquid-crystalline units that are comprised of fluid assemblies of amphiphilic molecules [3-5]. These membranes are commonly composed of a diverse group of lipids, and the lipid composition plays a critical role in membrane function and structure. Alterations in lipid composition or organization can have a profound impact on many cellular functions, including signal transduction and membrane trafficking $[6,7]$. Because biological membranes are so complex, to understand the role of a particular lipid component or stimulus on membrane function it is useful to perform experiments with model membranes [1, 8-10]. Model membranes have been used extensively in biophysical research because they often are made of a single, or a small variety of lipid components. A major components of lipid membranes is cholesterol, which is an important regulator of lipid organization. Sophisticated mechanisms are employed to maintain cholesterol levels in membranes in vivo [11], and the failure of these control mechanisms is associated with a variety of disease, such as atherosclerosis [12]. Cholesterol content is critical to the mechanical and physical properties of lipid bilayers, including: molecular fluidity [13, 14], compressibility [15], stiffness [16], resistance to shear stress [3], and thickness [10, 17]. Consequently, cholesterol plays a vital role in the regulation of physical and material properties of membranes, with pronounced consequences for cell physiology.

In this chapter, supported lipid bilayers, composed of total brain lipid extract (TBLE), were used as a model system to demonstrate how time-resolved tip/sample force interactions can be used to understand physical properties of membranes. Specifically, exogenous cholesterol was systematically incorporated into the supported TBLE bilayers to determine how cholesterol influences the mechanical properties of membranes. TBLE 
is an excellent model for cell membranes because it is comprised of a physiologically relevant ratio of membrane components, i.e. acidic and neutral phospholipids, gangliosides, cholesterol, sphingolipids, and isoprenoids (Fig. 1). For example, brain lipid extract has been used as a model surface in studying the interaction of amyloid proteins with membranes [14, 18-25] and as a mimic of the blood

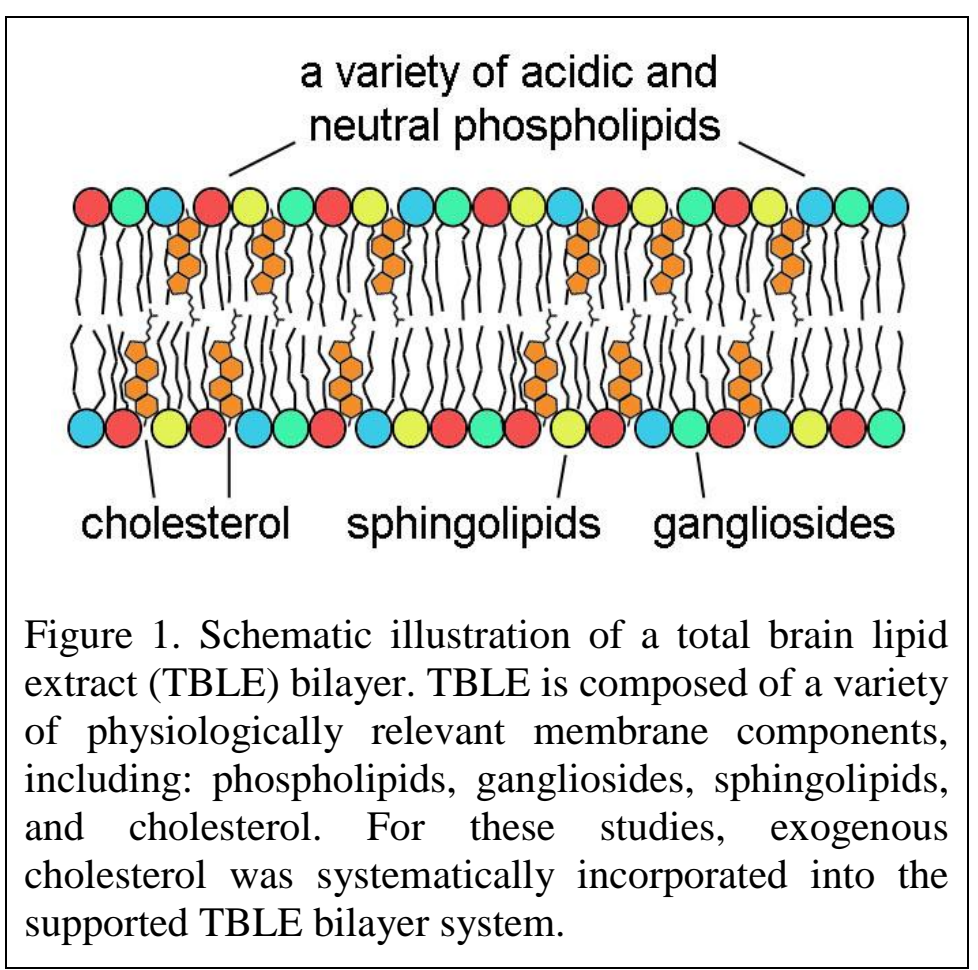

Figure 1. Schematic illustration of a total brain lipid extract (TBLE) bilayer. TBLE is composed of a variety of physiologically relevant membrane components, including: phospholipids, gangliosides, sphingolipids, and cholesterol. For these studies, exogenous cholesterol was systematically incorporated into the

brain barrier [26]. Additionally, the dependence of the tip/sample force interaction on the set-point ratio is briefly described, as understanding this relationship is critical to successfully compare results between experiments. SPAM experiments on a variety of supported TBLE/cholesterol bilayer systems are also presented, thereby providing insights into the local compressibility of the supported bilayer perpendicular to the plane of the surface and the adhesion of the supported bilayer with respect to the AFM tip. Finally, a supported TBLE bilayer containing 30\% exogenous cholesterol was heated, which resulted in the formation of lipid raft domains. This effect demonstrated that the SPAM technique is capable of achieving nanoscale lateral resolution when mapping the mechanical properties of supported lipid bilayers.

\subsection{Methodology}

\subsubsection{Sample preparation}

Total brain lipid extract (TBLE) (Avanti Polar Lipids) and cholesterol were dissolved in chloroform (ACROS Organics) and mixed at appropriate ratios based on the mass percentage. It was necessary to prepare samples according to mass percentage because approximately $60 \%$ of the lipid composition of TBLE is unknown (the relative 
concentrations of different components in TBLE are available from the Avanti Polar Lipids website). The chloroform was removed using a Vacufuge (Eppendorf). The resulting lipid films were resuspended in phosphate buffered saline (PBS, at $\mathrm{pH}$ 7.3) at a concentration of $1 \mathrm{mg} / \mathrm{ml}$. Bilayers and multilayer lipid sheets were formed by five sequential freeze-thaw cycles in liquid nitrogen. The lipid suspensions were then sonicated for 30 minutes at $40^{\circ} \mathrm{C}$ to promote vesicle formation. The suspended vesicle solution was diluted twenty times and $35 \mu \mathrm{L}$ was injected directly into the AFM fluid cell onto a freshly cleaved mica surface. Over time, small, patches of supported TBLE bilayers formed on the mica surface.

\subsubsection{AFM imaging conditions}

In situ AFM experiments were performed with a Nanoscope V Multimode scanning probe microscope (Veeco, Santa Barbara, CA) equipped with a tapping fluid cell and an O-ring. V shaped oxide-sharpened silicon nitride cantilevers were used throughout these studies, and had an advertised spring constant of $0.27 \mathrm{~N} / \mathrm{m}$ (Budget Sensors). While the manufacturer supplied the average value of the cantilever spring constant, the individual cantilevers used in this study had a spring constants ranging from 0.4 to $0.5 \mathrm{~N} / \mathrm{m}$ when calibrated in our lab. Images were acquired with the use of a closed-loop "vertical engage" J-scanner, and scan rates were set at $1.95 \mathrm{~Hz}$ with cantilever drive frequencies ranging from approximately $8-9 \mathrm{kHz}$. Images were captured at $5 \times 1.25 \mu \mathrm{m}$ and $512 \mathrm{x}$ 128 pixel resolutions. Images were captured at $5 \times 1.25 \mu \mathrm{m}$ and $512 \times 128$ pixel resolutions. The temperature of the system was controlled using the Bio-Heater accessory for the multimode AFM and monitored with a thermister incorporated into the fluid cell.

\subsubsection{Scanning probe acceleration microscopy analysis}

SPAM analysis was used to reconstruct every tapping event during AFM imaging as described in Chapter 2. Briefly, cantilever deflection trajectories were simultaneously captured during imaging through an AFM signal access module (Veeco) by using a CompuScope 14-Bit A/D Octopus data acquisition card (Gage Applied Technologies, Lachine, QB, Canada) and custom-written software. Trajectories were captured at 2-5 MS/s and 14-bit resolution with a resolution of 1-2 V. The trajectory of the cantilever 
was filtered using a Fourier transform based harmonic comb filter. Only intensities corresponding to integer harmonic frequencies were kept, and these were used to reconstruct a deflection signal, $y_{\text {rec }}(t)$. Once $y_{\text {rec }}(t)$ was obtained, the second derivative of the cantilever trajectory was taken and multiplied by the effective mass, $m_{\text {eff }}$, of the cantilever to obtain the time-resolved based tapping force between the tip and sample.

\subsection{Results and discussion}

\subsubsection{The role of set-point on time-resolved tip/sample force}

While the simulations presented in Chapter 2 demonstrated that the maximum and minimum tapping forces ( $F_{\max }$ and $F_{\min }$, respectively) can be useful indicators of the mechanical properties of surfaces, to make comparisons across samples, it is critical to understand the impact of imaging conditions (such as set-point ratio) on the time-resolved tip/surface tapping force. To determine how the relative magnitude of $F_{\max }$ and $F_{\min }$ changed with varying the set-point ratio, experiments were performed on the supported TBLE bilayer patches on (Fig. 2). Throughout this study, the temperature was maintained between 23 and $24{ }^{\circ} \mathrm{C}$ to prevent any variations in the phase of the supported lipid bilayer patch. Patches of bilayer were used in this study rather than a complete bilayer so that the exposed mica surface could serve as an internal reference to study the relative values of tapping forces on the supported TBLE bilayer. Supported TBLE bilayers have a considerably lower elastic modulus than mica, so this system provides a surface with a soft region (the bilayer patch) and a hard region (the exposed mica). The mean distance between the height of the mica substrate $(\sim 0 \mathrm{~nm})$ and the top of the supported bilayer ( 3.6-3.9 $\mathrm{nm})$ did not appreciably or systematically change as a function of set-point ratio, thus the set-point ratio had no discernible effect on the measured height of the supported TBLE bilayer patches (Fig. 2a-b). However, in every image, the measured height of the supported bilayer was smaller than the typical height as one would expect for an uncompressed TBLE bilayer $(\sim 5 \mathrm{~nm})$, indicating that the supported bilayer was compliant under the applied imaging force of the probe. As demonstrated by the simulations in Chapter 2, the $F_{\max }$ associated with imaging regions of different rigidity increased with increasing elastic modulus; therefore, regions of the surface with varying elastic modulus can be indicated by surface maps of the maximum tapping force (Fig. 
2c). As expected, the magnitude of $F_{\max }$ was smaller in regions associated with the supported bilayer, indicating that the supported bilayer was softer than the mica. Contrast was also observed in surface maps of $F_{\text {min }}$ for the supported bilayer patch/mica system (Fig. 2d), and this contrast between supported bilayer and mica can be attributed to a combination of differing surface rigidity and adhesion to the tip.

Although the measured height of the supported bilayer patches were not altered as a function of set-point ratio, the magnitudes of $F_{\max }$ and $F_{\min }$ changed with decreasing setpoint ratio (Fig. 2e-f). Histograms of the forces for each tapping event associated with obtaining the image were bimodal, where the two peaks in each $F_{\max }$ and $F_{\min }$ histogram correspond to the mica and supported bilayer portions of the surface. In the $F_{\max }$ images

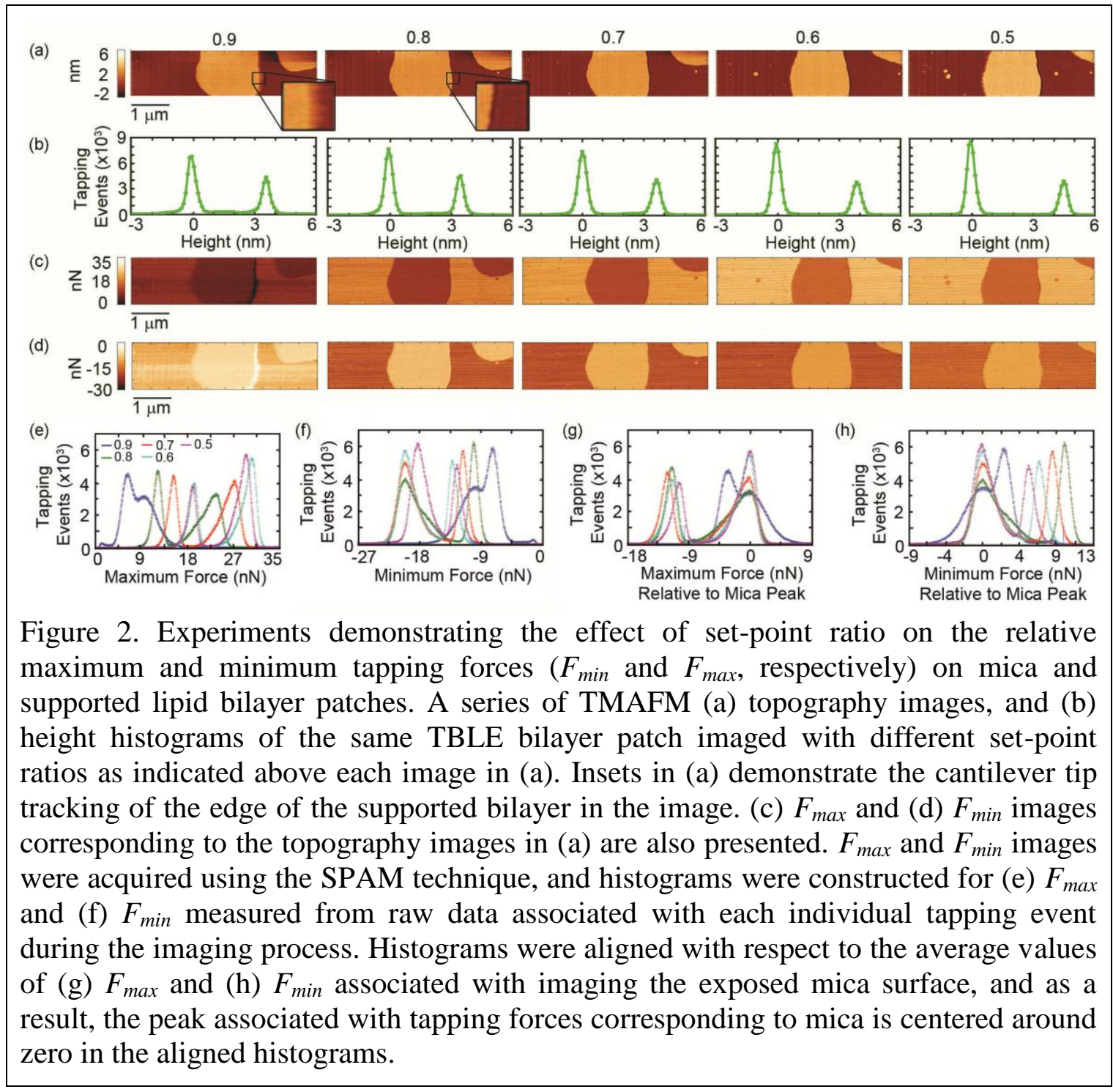


and histograms, the larger magnitude force is associated with mica, and for the $F_{\min }$ images and histograms, the larger magnitude (more negative) $F_{\min }$ is also associated with mica. When the set-point ratio was lowered, the applied total force per tapping event to the surface increased, which resulted in larger values of $F_{\max }$ (Fig. 2e). This result is consistent with previously reported simulations and experiments [27]. However, when the force histograms were normalized to the value of $F_{\max }$ associated with mica, the relative value of $F_{\max }$ on the supported bilayer patch with respect to mica was maintained for setpoint ratios of $0.5-0.8$ (Fig. $2 \mathrm{~g}$ ). At a set-point ratio of 0.9 , the relative value of $F_{\max }$ associated with supported bilayer with respect to mica was smaller than the values of $F_{\max }$ associated with the supported bilayer at lower set-points; at a set-point of 0.9, the tip barely makes contact with the surface, thereby reducing the physical interaction between the cantilever tip and the sample surface. This effect is further supported by evidence in the height images (Fig. 2a insets), where the tip is unable to properly track the edges of the supported bilayer with a set-point ratio of 0.9 , as compared to lower values of setpoint ratio. Thus, once a certain set-point ratio is reached, the relative tip-surface interactions associated with $F_{\max }$ on surfaces of varying elasticity remains constant. This observation makes comparisons between samples easy as long as an internal reference surface, such as mica, is present. This simplifies calibration of such images with respect to surface properties. The raw magnitude of $F_{\min }$ associated with mica does not appreciably change. Once the set-point has been reduced below 0.9 (Fig. 2f), however, the value of $F_{\min }$ associated with the supported bilayer becomes larger in magnitude. When the force histograms were normalized to value of $F_{\min }$ associated with mica, the relative value of $F_{\min }$ for the supported bilayer with respect to mica systematically decreased as the set-point ratio was reduced from 0.8 to 0.5 (Fig. 2h). This demonstrates that the relative values of $F_{\min }$ are highly dependent on the set-point ratio, and one must be careful to maintain the same set-point ratio when imaging to facilitate comparison of $F_{\min }$ values between separate samples. Similar to $F_{\max }$, the relative values of $F_{\min }$ associated with bilayer and mica with a set-point ratio of 0.9 does not follow the pattern because the tip was barely tracking the surface. 


\subsubsection{Physical and mechanical properties of TBLE supported bilayers change as a function of cholesterol content}

To determine whether the time-resolved tip/sample tapping force could be used to detect physical changes in supported bilayers associated with lipid composition, a series of experiments were performed on supported TBLE bilayer patches containing varying amounts of exogenous cholesterol. Conventionally lipid concentrations in mixtures are determined by molar ratios; however, this study determined cholesterol composition by mass percentage because TBLE is a complex mixture of lipids where approximately $60 \%$ of the extract is made of unknown lipid species. The samples utilized in this study were: 1) $100 \%$ TBLE, 2) $90 \%$ TBLE $+10 \%$ exogenous cholesterol, 3) $80 \%$ TBLE $+20 \%$ exogenous cholesterol, 4) 70\% TBLE $+30 \%$ exogenous cholesterol, 5) $60 \%$ TBLE + $40 \%$ exogenous cholesterol, and 6) 50\% TBLE + 50\% exogenous cholesterol. By systematically enriching the bilayer with exogenous cholesterol, we can evaluate the effectiveness of the SPAM technique to measure changes in the material properties of supported bilayers due to changes in lipid composition. Similar to the previous studies examining the effect of set-point ratio, the temperature of the system was maintained at 23 to $24{ }^{\circ} \mathrm{C}$. Additionally, based on the findings from previous studies and the set-point experiments, to facilitate comparisons between samples, it was critical to maintain imaging parameters, including: set-point ratio (0.8), a constant drive amplitude, and free amplitude. To simplify the situation, the same cantilever was used for each experiment; however, because this experiment involved imaging six different samples, it was necessary to clean the AFM fluid cell and cantilever after evaluating each TBLE/cholesterol system. Thus, caution was practiced when cleaning the system and changing the samples to avoid damaging the cantilever. By using the same cantilever,

relatively similar operating frequencies and maintaining a constant drive amplitude and set-point ratio, it was possible to compare forces across experiments because the total tip/sample force per oscillation cycle is given by:

$$
F_{\text {total }} \approx 0.5 k a_{0} \frac{\Delta A}{A_{0}}
$$


where $k$ is the cantilever spring constant, $a_{0}$ is the drive amplitude, $\Delta A$ is the tapping amplitude and $A_{0}$ is the free amplitude [28]. As previously described, the forces associated with imaging the exposed mica were used as an internal reference to normalize small variations in the tip/sample forces, making it possible to compare relative shifts in the magnitude of tapping forces [27]. At any given free amplitude and set-point ratio, the height of the bilayer patches with respect to the underlying mica substrate changed as a function of addition of cholesterol (Fig. 3). Pure TBLE had a measured height of $3.6 \mathrm{~nm}$, which was consistent with those observed for the set-point ratio experiments shown in

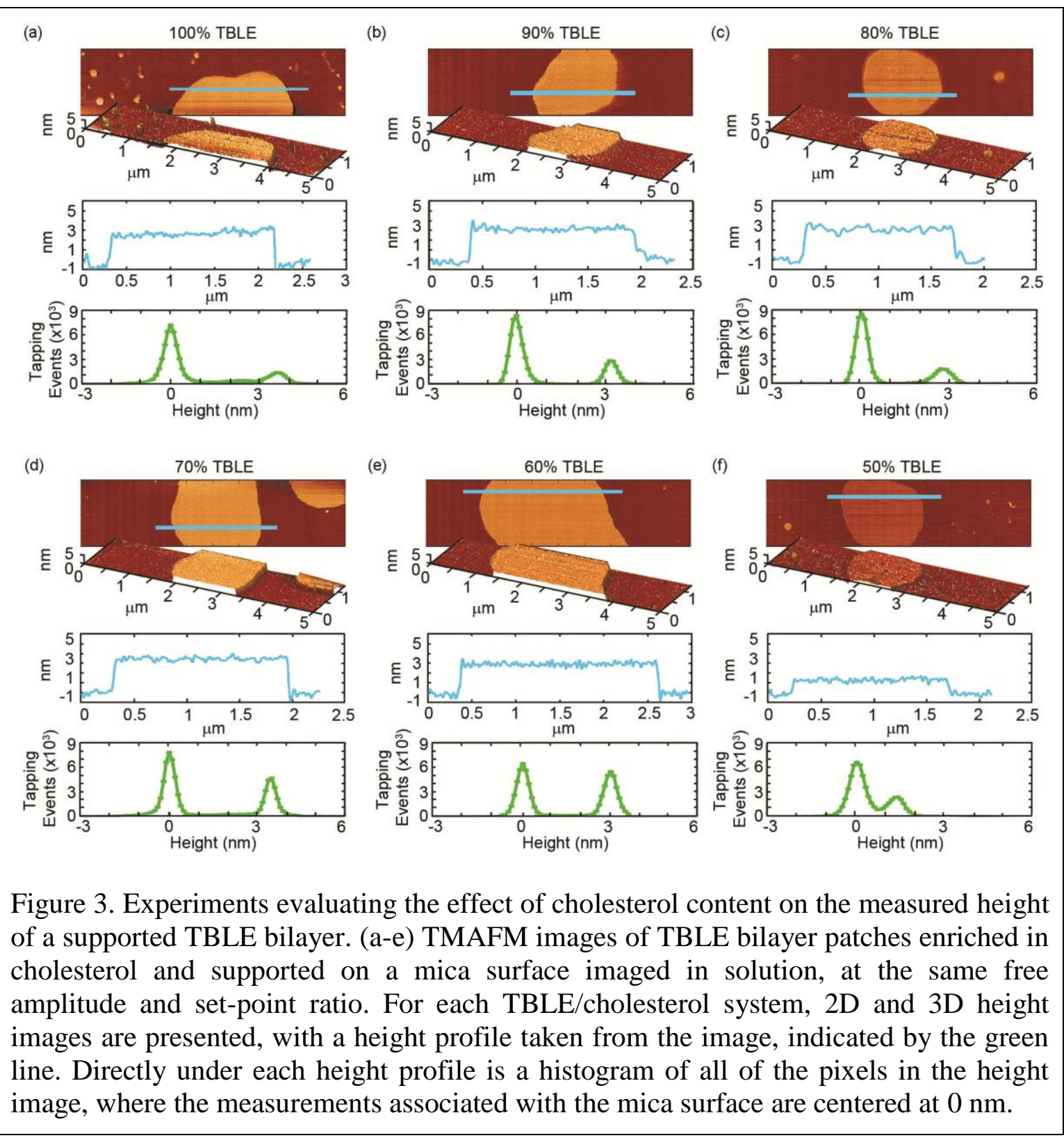


Fig. 2. As the exogenous cholesterol was increased from $0 \%$ to $20 \%$, the measured height of the bilayer systematically decreased from 3.6 to $2.7 \mathrm{~nm}$. At $30 \%$ exogenous cholesterol, the measured height of the bilayer increased to $3.5 \mathrm{~nm}$. Adding more cholesterol to the bilayer caused the mean height of the bilayer to drop to 3.0 and $1.4 \mathrm{~nm}$ for $40 \%$ and $50 \%$ exogenous cholesterol, respectively. Based on simulations described in Chapter 2, changes in the Young's modulus or Hamaker constant associated with adding cholesterol to the bilayers could have caused changes in the measured height of the bilayer. The significantly decreased measured height for the TBLE bilayer containing $50 \%$ exogenous cholesterol could have been caused by several factors. One possibility is that the supported bilayer was approaching its cholesterol saturation point, thereby preventing efficient lipid packing into the ordered bilayer [9]. Studies have reported that when cholesterol concentrations in the bilayer are equal to or greater than 50\%, cholesterol is insoluble in the bilayer and precipitation can occur [15, 29]. Therefore, the increased compressibility may be attributed to the inability of the cholesterol molecules to be fully incorporated into the bilayer. The shorter cholesterol molecules within the bilayer, which are known to have a length of about $2 \mathrm{~nm}$ [30,31], may also be contributing to the shorter observed bilayer patches. The thickness of the bilayers changes due to the addition of exogenous cholesterol, with the bilayer thickness increasing initially with additional cholesterol before reaching a maximum thickness and decreasing [17]. Another possibility for the height differences is a cholesterol induced phase transition in the lipid bilayer.

To determine the dominant contributor to the observed bilayer heights as a function of cholesterol content, mechanical surface maps of $F_{\max }$ and $F_{\min }$ were constructed using the SPAM technique (Fig. 4-5). These mechanical maps directly correspond to the topography images (Fig. 3). While $F_{\max }$ is a measure of the surfaces local Young's modulus, $F_{\min }$ is related to the force required to break contact between the tip and surface, i.e. adhesion, and can be associated with local changes in surface free energy, as demonstrated by simulations in Chapter 2. Similar to the set-point experiments, there was a bimodal distribution of data in histograms of $F_{\max }$ and $F_{\min }$ associated with the TBLE bilayer and mica surfaces. In the $F_{\max }$ images and histograms, the smaller positive force is associated with the lipid bilayers (which was variable for the 
different bilayer systems), indicating that the bilayers have a lower elastic modulus than the mica substrate $\left(F_{\max } \sim 35 \mathrm{nN}\right)$. The smaller value of $F_{\min }$ is associated with the lipid bilayers, indicating that the tip-supported bilayer interaction (which had variable $F_{\min }$ values depending on cholesterol content) has a smaller surface free energy compared to the tip-mica substrate interaction $\left(F_{\min } \sim 28.5 \mathrm{nN}\right)$. The shift in the observed magnitudes of $F_{\max }$ and $F_{\min }$ for bilayers with respect to the mica surface indicates that the addition of cholesterol altered the mechanical properties of the supported bilayers (histograms in Fig. 4 and 5).

Features of the time-resolved tip/sample tapping forces on the different bilayer systems can be directly compared because the cantilever (i.e., same spring constant and mass) and imaging parameters (i.e., drive amplitude, free amplitude, and set-point ratio) were maintained in all experiments (Fig. 6). Smaller values of $F_{\max }$ associated with tapping events on the bilayer correspond to a lower elastic modulus after taking into
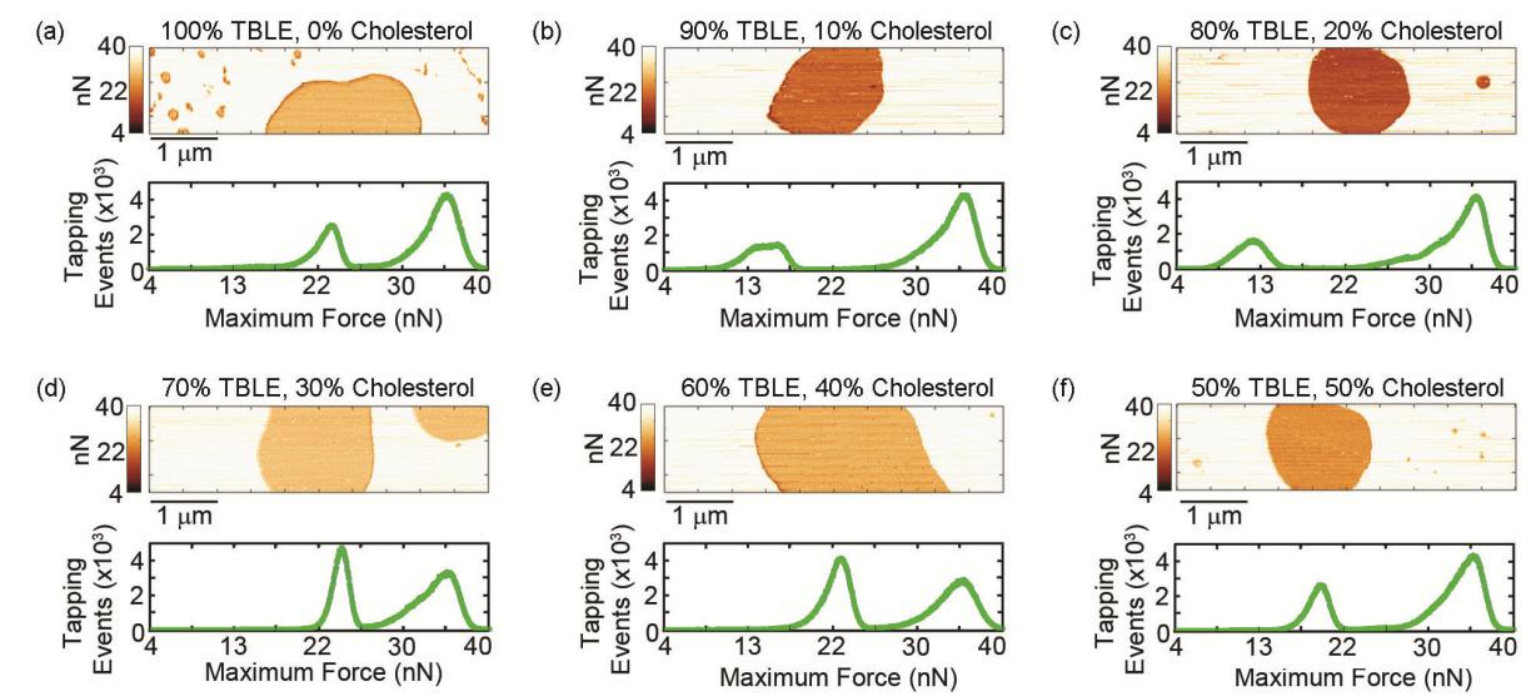

Figure 4. Reconstructed maximum tapping force $\left(F_{\max }\right)$ images of TBLE/cholesterol bilayer patches supported on a mica surface. $F_{\max }$ images associated with (a) $100 \%$ TBLE, (b) 90\% TBLE and 10\% cholesterol, (c) 80\% TBLE and 20\% cholesterol, (d) $70 \%$ TBLE and 30\% cholesterol, (e) 60\% TBLE and 40\% cholesterol, and (f) 50\% TBLE and $50 \%$ cholesterol are presented. These images directly correspond to the height images presented in Fig. 3. Histograms of $F_{\max }$ constructed from each tapping event of the imaging process are provided directly below each image, where there is a bimodal distribution of tapping forces. The peaks associated with the tapping events on the mica surface are normalized to $\sim 35 \mathrm{nN}$, and the peaks associated with tapping events on the lipid bilayer patch are to the left. 

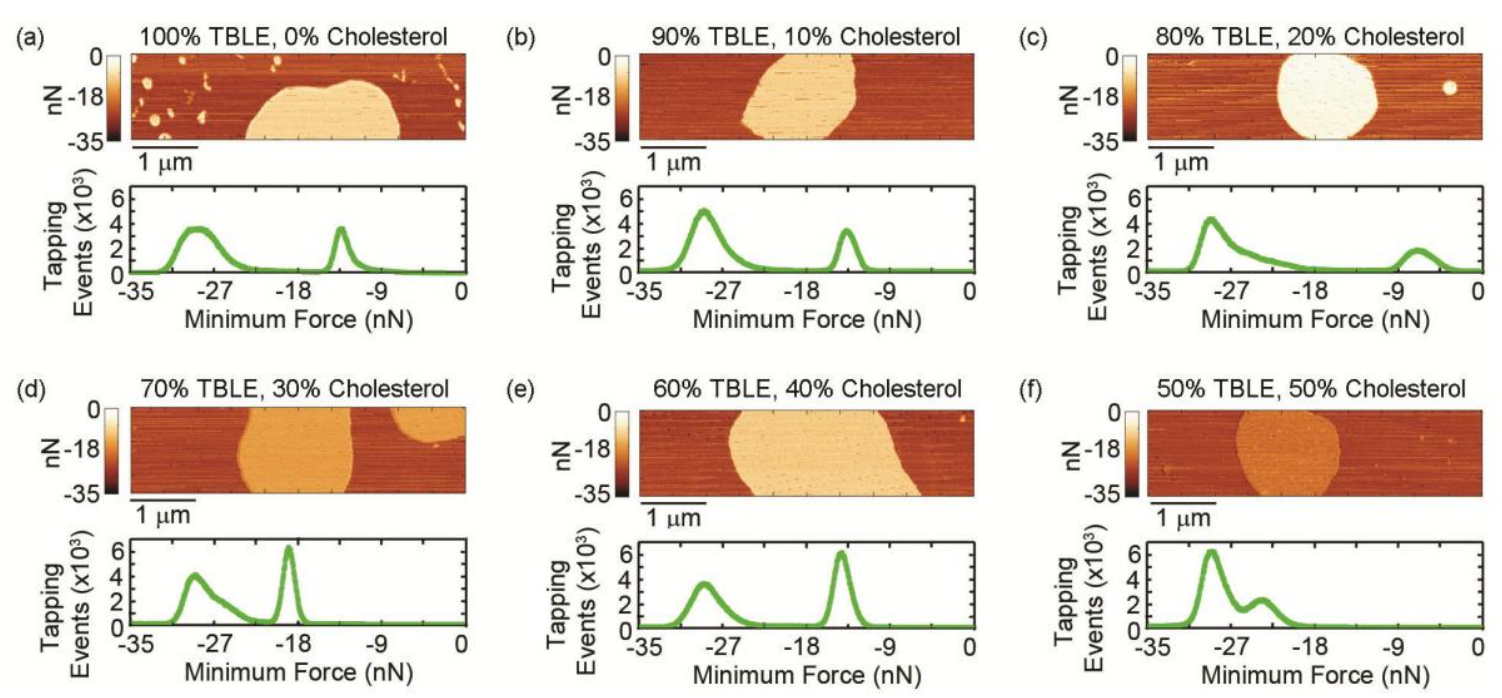

Figure 5. Reconstructed minimum tapping force $\left(F_{\min }\right)$ images of TBLE/cholesterol bilayer patches supported on a mica surface. $F_{\min }$ images associated with (a) $100 \%$ TBLE, (b) 90\% TBLE and 10\% cholesterol, (c) 80\% TBLE and 20\% cholesterol, (d) $70 \%$ TBLE and 30\% cholesterol, (e) 60\% TBLE and 40\% cholesterol, and (f) $50 \%$ TBLE and $50 \%$ cholesterol are presented. These images directly correspond to the height images presented in Fig. 3. Histograms of $F_{\min }$ constructed from each tapping event of the imaging process are provided directly below each image, where there is a bimodal distribution of tapping forces. The peaks associated with the tapping events on the mica surface are normalized to $\sim-28.5 \mathrm{nN}$, and the peaks associated with tapping events on the lipid bilayer patch are to the right.

account any contributions due to any increased attractive interaction, which can be estimated based on $F_{\min }$. Initially, there was a decrease in $F_{\max }$ as the cholesterol content of the bilayer was increased from $0 \%$ to $20 \%$ (Fig. 6a). This trend was followed by a dramatic increase in $F_{\max }$ when $30 \%$ exogenous cholesterol was added to the supported bilayers ( $p<0.01$ when comparing $20 \%$ exogenous cholesterol to $30 \%$ exogenous cholesterol content). Although there was no significant change observed in $F_{\max }$ when the cholesterol content was increased from $30 \%$ to $40 \%$, another significant decrease ( $p<$ 0.05 ) in $F_{\max }$ was observed when exogenous cholesterol was added (up to 50\%). This bimodal pattern indicates an initial decrease in Young's modulus of the bilayer with the addition of cholesterol, followed by a sharp increase in the Young's modulus, followed with another decrease. Interestingly, the observed heights of the bilayers with $0 \%$ to $40 \%$ exogenous cholesterol correspond well with the magnitude of the associated magnitude of $F_{\max }$. That is, the bilayers with the smallest values of $F_{\max }$, which should be the most 

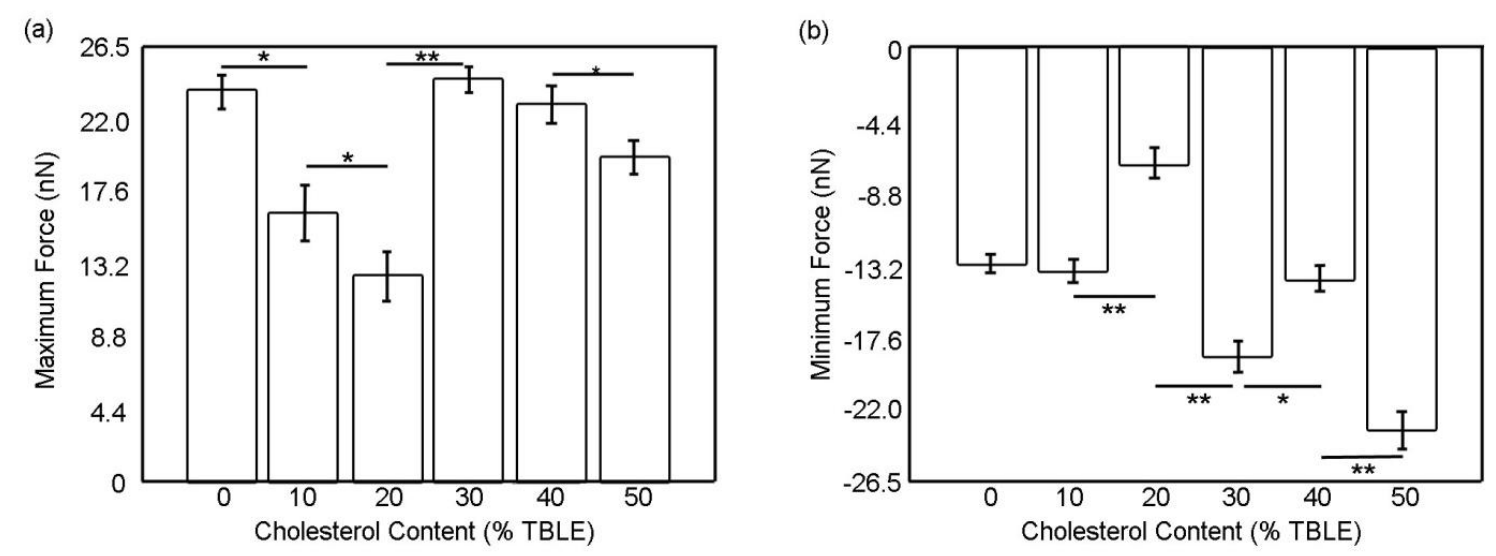

Figure 6. Average values of the (a) maximum and (b) minimum tapping forces on supported TBLE bilayer patches as a function of exogenous cholesterol content. The average values were calculated from each tapping event that occurred on the bilayer patch, and the error bars represent the standard deviation. * indicates $p<0.05$, and ** indicates $p<0.01$ based on a simple $t$ test.

easily compressed based on the associated relative value of Young's modulus, have smaller observed heights. This relationship did not hold true for the bilayer containing $50 \%$ exogenous cholesterol, which again may be due partially to the fact that the bilayer is near its cholesterol saturation point [9]. The bimodal pattern of the values of $F_{\max }$ associated with the bilayer as a function of cholesterol content is intriguingly similar to the pattern observed by Yip et al. for membrane fluidity on the same system using fluorescence DPH (diphenylhexatriene) anisotropy [14]. That study demonstrated that the membrane fluidity decreased with addition of exogenous cholesterol to TBLE up to $20 \%$, had a maximum lateral fluidity at 30\% exogenous cholesterol, and decreased again with the further addition of cholesterol to the TBLE bilayer [14]. A caveat of this comparison is that the fluorescence DPH anisotropy measurements were made on lipid vesicles rather than the supported lipid bilayer systems used in these studies. The fluidity of the supported lipid bilayer can be influenced by the mica substrate, thereby complicating comparisons between the two types of studies. Nonetheless, the similar trend observed in both cases eludes to interesting relationship between the lateral fluidity and vertical compressibility of lipid membranes in which the larger the lateral fluidity of the membrane, the larger the Young's modulus. Such a scenario would be advantageous for biological membranes that need to balance lateral mobility within the membrane to 
maintain structural integrity of the membrane as a whole. Other studies have reported that molecules, such as cholesterol, which lie parallel to a lipid chain will experience only slight perturbations if pressure is applied in the vertical direction; however the horizontal compressibility can be much greater [32], which may partially explain the observed relationship between vertical compressibility and lateral fluidity of a lipid bilayer. Similar increases in bilayer compression perpendicular to the membrane plane have been observed in other simpler bilayer systems, but the exact changes in elasticity due to addition of cholesterol are dependent on the nature of the phospholipids [15]. Another potential factor contributing to the observed changes in relative Young's modulus from the addition of exogenous cholesterol could be due to changes in phase of the supported bilayer, as cholesterol has been shown to induce transitions from the liquid disordered to the liquid ordered phase in POPC vesicles [33]. There is biological relevance associated with such cholesterol-induced phase transitions, as the membrane structure associated with the lipid phase appears to be the determining factor in the pore-forming activity of the polyene antibiotic nystatin on POPC/cholesterol vesicles [34].

As demonstrated by simulations in Chapter $2, F_{\min }$ provides information about the adhesive force between the AFM probe and bilayer surface. Specifically, the larger the magnitude of $F_{\text {min }}$, the more adhesive the surface is to the cantilever tip. $F_{\text {min }}$ as a function of cholesterol content did not demonstrate the same pattern as produced by $F_{\max }$ (Fig. 6b) or the bilayer fluidity data from Yip et al. [14]. With the addition of $10 \%$ exogenous cholesterol to the bilayer, the adhesion between the bilayer and the tip did not appreciably change. At $20 \%$ exogenous cholesterol, the adhesive force between the tip and the bilayer decreased significantly $(p<0.01)$, but the adhesion became much stronger with the addition of $30 \%$ cholesterol $(p<0.01)$. The adhesive force at $40 \%$ cholesterol was similar to that of a pure TBLE bilayer and one containing 10\% cholesterol; however, this represented a significant decrease in the adhesive force when comparing samples containing 30\% exogenous cholesterol. An interesting feature was observed with the bilayer containing 50\% exogenous cholesterol, the adhesive force was significantly ( $p<$ 0.01) increased as compared to the other TBLE/cholesterol compositions. As simulations presented in Chapter 2 have demonstrated, large adhesive forces can result in an increased compression of soft surfaces, and this large increase in adhesion could also 
contribute to the lower observed height as measured by AFM. This could further explain why the measured height of the bilayer containing 50\% exogenous cholesterol was an outlier to the observed relationship between $F_{\max }$ and measured bilayer height. A potential complication of this type of analysis is that lipids can adhere to the cantilever tip; however, based on the topography images, there is no evidence that this occurred in any of the presented experiments. To avoid this complication, the AFM fluid cell and the cantilever were cleaned between experiments (imaging each of the six TBLE/cholesterol mixtures). Additionally, if a layer of lipids would have formed the cantilever tip, the images would have had evidence of tapping on a double bilayer. TMAFM experiments by $\mathrm{Xu}$ et al. demonstrated that compositional contrast in higher harmonic images on supported purple membranes that were one or two layers thick [35]; therefore, we suspect that the tapping forces would be altered by a lipid layer formed on the surface of the tip, because double bilayers are more compressible than single bilayers.

\subsubsection{Material properties of lipid rafts are distinguishable from the surrounding supported lipid bilayer}

To demonstrate the ability of SPAM to map the mechanical properties of a surface with nanoscale spatial resolution, supported TBLE bilayers with $30 \%$ exogenous cholesterol were heated above $35^{\circ} \mathrm{C}$ to induce the formation of nanoscale lipid domains. Heating the system caused a reduction in bilayer height to $\sim 2.4 \mathrm{~nm}$ above the mica surface (Fig. 7a), and lipid rafts were formed within the domain. These domains were $\sim 0.7-1.2 \mathrm{~nm}$ shorter in height compared to the surrounding bilayer, and the rafts had diameters ranging from $\sim 75-250 \mathrm{~nm}$ (based on the topographical image in Fig. 7b). The supported TBLE bilayers with rafts were imaged, and the forces associated with each tapping event were reconstructed using the SPAM technique to obtain $F_{\max }$ and $F_{\min }$ images (Fig. 7c and 7d, respectively). The lipid raft domains were associated with an increase in magnitude of $F_{\max }$ and $F_{\min }$ in comparison to the surrounding lipid bilayer. Although the change in $F_{\max }$ may be partially due to an increased rigidity of the raft domains, the relative shifts associated with $F_{\max }$ and $F_{\min }$ were comparable, indicating that the changes in adhesive properties of the supported bilayer are most likely the dominant factor in the observed tapping forces (both $F_{\max }$ and $F_{\min }$ ). Importantly, this experiment demonstrates that 
SPAM is capable of achieving lateral resolution of surface mechanical maps on the nanoscale; however, similar to TMAFM experiments, the lateral resolution of the SPAM process appears to be limited by the size of the cantilever tip and therefore, the contact area between the tip and the surface.

\subsection{Conclusions}

An important direction in the field of scanning probe microscopy is the enhancement and further development of methods to simultaneously capture topographical and mechanical information about surfaces. Reconstruction of the timeresolved tip/sample tapping (a)
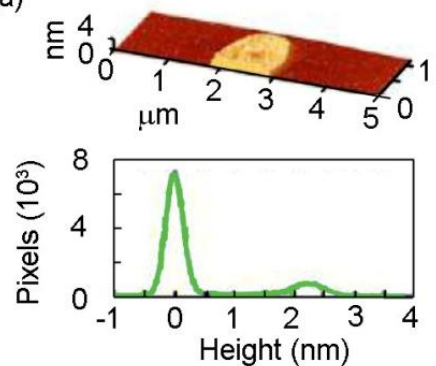

(c)

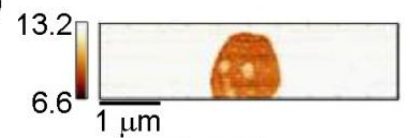

— Lipid Bilayer - Raft Domain

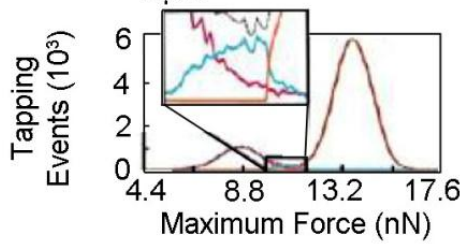

(b)
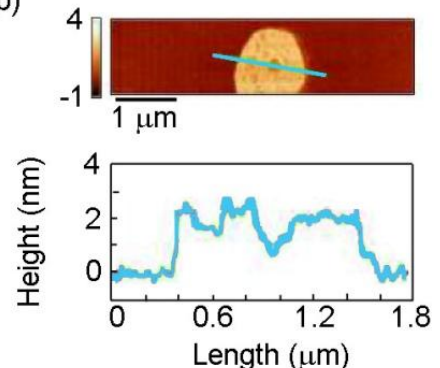

(d)

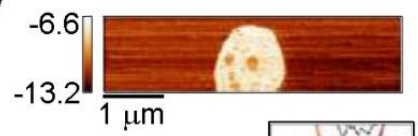

Figure 7. Lipid raft domains are formed in TBLE/cholesterol systems at elevated temperatures. (a) 3D TMAFM topography image and height histogram of a supported TBLE bilayer containing 30\% exogenous cholesterol taken at $35{ }^{\circ} \mathrm{C}$, where raft domains are visible. (b) The green line in the height image corresponds to the height profile below, demonstrating that the raft domains were $\sim 0.7-1.2 \mathrm{~nm}$ lower in height than the surrounding bilayer, and had diameters of $\sim 75-$ $250 \mathrm{~nm}$. Reconstructed, spatially resolved images and corresponding histograms of (c) $F_{\max }$ and (d) $F_{\min }$. The forces associated with different regions of the surfaces (lipid bilayer, raft domain, and mica) were sorted in the histograms based on topography, and the insets magnify the regions of the associated with the raft domains.

force during standard TMAFM operation is an attractive method for achieving this goal, and several techniques have been developed to accomplish the recovery of these forces $[2,36-44]$. As demonstrated by simulations in Chapter 2 and experimental results, the maximum and minimum tapping forces for TMAFM operation in fluids can be related to material properties of surfaces such as Young's modulus and the surface free energy between the cantilever tip and sample surface. The reconstructed tip/sample force interactions can provide insight into the mechanical properties of biologically relevant 
surfaces with nanoscale spatial resolution. SPAM is a technique which enables the reconstruction of tapping forces from the deflection signal of a TMAFM experiment; here, the applicability of SPAM to investigate the mechanical changes induced in supported TBLE bilayer patches enriched with exogenous cholesterol was demonstrated. In this particular study, maximum tapping forces provided evidence for a link between the vertical compressibility and lateral fluidity of a membrane, as demonstrated using TBLE bilayers containing various amounts of exogenous cholesterol. Additionally, minimum tapping forces provided information about adhesive properties of the lipid bilayer to the cantilever tip. To facilitate comparison between samples, it was imperative to hold several key parameters constant, such as drive amplitude, free amplitude, and setpoint ratio. To simplify this issue, the same cantilever was used for all experiments. Importantly, all of these experiments were performed under physiologically relevant buffer conditions, which makes the SPAM technique particularly useful for studying biologically relevant surfaces.

\subsection{References}

1. Li, J.K., R.M.A. Sullan, and S. Zou, Atomic force microscopy force mapping in the study of supported lipid bilayers. Langmuir, 2011. 27(4): p. 1308-1313.

2. Legleiter, J., M. Park, B. Cusick, and T. Kowalewski, Scanning probe acceleration microscopy (SPAM) in fluids: Mapping mechanical properties of surfaces at the nanoscale. Proceedings of the National Academy of Sciences of the United States of America, 2006. 103(13): p. 4813-4818.

3. Espinosa, G., I. Lopez-Montero, F. Monroy, and D. Langevin, Shear rheology of lipid monolayers and insights on membrane fluidity. Proceedings of the National Academy of Sciences of the United States of America, 2011. 108(15): p. 60086013.

4. Singer, S.J. and G.L. Nicolson, Fluid mosaic model of structure of cell membranes. Science, 1972. 175(4023): p. 720-\&.

5. van Meer, G., D.R. Voelker, and G.W. Feigenson, Membrane lipids: where they are and how they behave. Nature Reviews Molecular Cell Biology, 2008. 9(2): p. 112-124. 
6. Mukherjee, S. and F.R. Maxfield, Membrane domains. Annual Review of Cell and Developmental Biology, 2004. 20: p. 839-866.

7. Simons, K. and W.L.C. Vaz, Model systems, lipid rafts, and cell membranes. Annual Review of Biophysics and Biomolecular Structure, 2004. 33: p. 269-295.

8. Evans, E. and D. Needham, Physical properties of surfactant bilayer membranesthermal transitions, elasticity, rigidity, cohesion, and colloidal interactions. Journal of Physical Chemistry, 1987. 91(16): p. 4219-4228.

9. Needham, D. and R.S. Nunn, Elastic deformation and failure of lipid bilayer membranes containing cholesterol. Biophysical Journal, 1990. 58(4): p. 9971009.

10. Pan, J., T.T. Mills, S. Tristram-Nagle, and J.F. Nagle, Cholesterol perturbs lipid bilayers nonuniversally. Physical Review Letters, 2008. 100(19).

11. Goldstein, J.L. and M.S. Brown, Molecular medicine - The cholesterol quartet. Science, 2001. 292(5520): p. 1310-1312.

12. Maxfield, F.R. and I. Tabas, Role of cholesterol and lipid organization in disease. Nature, 2005. 438(7068): p. 612-621.

13. Asakawa, H. and T. Fukuma, The molecular-scale arrangement and mechanical strength of phospholipid/cholesterol mixed bilayers investigated by frequency modulation atomic force microscopy in liquid. Nanotechnology, 2009. 20(26).

14. Yip, C.M., E.A. Elton, A.A. Darabie, M.R. Morrison, and J. McLaurin, Cholesterol, a modulator of membrane-associated amyloid $\beta$-fibrillogenesis and neurotoxicity. Journal of Molecular Biology, 2001. 311(4): p. 723-734.

15. Hianik, T., M. Haburcak, K. Lohner, E. Prenner, F. Paltauf, and A. Hermetter, Compressibility and density of lipid bilayers composed of polyunsaturated phospholipids and cholesterol. Colloids and Surfaces a-Physicochemical and Engineering Aspects, 1998. 139(2): p. 189-197.

16. Byfield, F.J., H. Aranda-Espinoza, V.G. Romanenko, G.H. Rothblat, and I. Levitan, Cholesterol depletion increases membrane stiffness of aortic endothelial cells. Biophysical Journal, 2004. 87(5): p. 3336-3343.

17. Hung, W.-C., M.-T. Lee, F.-Y. Chen, and H.W. Huang, The condensing effect of cholesterol in lipid bilayers. Biophysical Journal, 2007. 92(11): p. 3960-3967. 
18. Legleiter, J., J.D. Fryer, D.M. Holtzman, and T. Kowalewski, The modulating effect of mechanical changes in lipid bilayers caused by ApoE-containing lipoproteins on amyloid $\beta$ induced membrane disruption. ACS Chemical Neuroscience, 2011. 2(10): p. 588-599.

19. Pifer, P.M., E.A. Yates, and J. Legleiter, Point mutations in amyloid $\beta$ result in the formation of distinct polymorphic aggregates in the presence of lipid bilayers. Plos One, 2011. 6(1).

20. Chaibva, M., K.A. Burke, and J. Legleiter, Curvature enhances binding and aggregation of huntingtin at lipid membranes. Biochemistry, 2014. 53(14): p. 2355-2365.

21. Burke, K.A., K.M. Hensal, C.S. Umbaugh, M. Chaibva, and J. Legleiter, Huntingtin disrupts lipid bilayers in a polyQ-length dependent manner. Biochimica Et Biophysica Acta-Biomembranes, 2013. 1828(8): p. 1953-1961.

22. Burke, K.A., K.J. Kauffman, C.S. Umbaugh, S.L. Frey, and J. Legleiter, The interaction of polyglutamine peptides with lipid membranes is regulated by flanking sequences associated with huntingtin. Journal of Biological Chemistry, 2013. 288(21): p. 14993-15005.

23. Yates, E.A., S.L. Owens, M.F. Lynch, E.M. Cucco, C.S. Umbaugh, and J. Legleiter, Specific domains of amyloid $\beta$ facilitate aggregation on and association with lipid bilayers. Journal of Molecular Biology, 2013. 425(11): p. 1915-1933.

24. Burke, K.A., E.A. Yates, and J. Legleiter, Amyloid-forming proteins alter the local mechanical properties of lipid membranes. Biochemistry, 2013. 52(5): p. 808-817.

25. Burke, K.A., E.A. Yates, and J. Legleiter, Biophysical insights into how surfaces, including lipid membranes, modulate protein aggregation related to neurodegeneration. Frontiers in Neurology, 2013. 4.

26. Tsinman, O., K. Tsinman, N. Sun, and A. Avdeef, Physicochemical selectivity of the BBB microenvironment governing passive diffusion-matching with a porcine brain lipid extract artificial membrane permeability model. Pharmaceutical Research, 2011. 28(2): p. 337-363. 
27. Kumar, B., P.M. Pifer, A. Giovengo, and J. Legleiter, The effect of set point ratio and surface Young's modulus on maximum tapping forces in fluid tapping mode atomic force microscopy. Journal of Applied Physics, 2010. 107(4).

28. Kowalewski, T. and J. Legleiter, Imaging stability and average tip-sample force in tapping mode atomic force microscopy. Journal of Applied Physics, 2006. 99(6).

29. Ibarguren, M., A. Alonso, B.G. Tenchov, and F.M. Goni, Quantitation of cholesterol incorporation into extruded lipid bilayers. Biochimica Et Biophysica Acta-Biomembranes, 2010. 1798(9): p. 1735-1738.

30. Craven, B.M., Crystal structure of cholesterol monohydrate. Nature, 1976. 260(5553): p. 727-729.

31. Sankaram, M.B. and T.E. Thompson, Modulation of phospholipid acyl chain order by cholesterol- a solid state $\mathrm{H} 2$ nuclear magnetic resonance study. Biochemistry, 1990. 29(47): p. 10676-10684.

32. Scarlata, S.F., Compression of lipid membranes as observed at varying membrane positions. Biophysical Journal, 1991. 60(2): p. 334-340.

33. Silva, L., A. Coutinho, A. Fedorov, and M. Prieto, Competitive binding of cholesterol and ergosterol to the polyene antibiotic nystatin. A fluorescence study. Biophysical Journal, 2006. 90(10): p. 3625-3631.

34. Gonzalez-Damian, J. and I. Ortega-Blake, Effect of membrane structure on the action of polyenes II: nystatin activity along the phase diagram of ergosterol-and cholesterol-containing POPC membranes. Journal of Membrane Biology, 2010. 237(1): p. 41-49.

35. Xu, X., J. Melcher, S. Basak, R. Reifenberger, and A. Raman, Compositional contrast of biological materials in liquids using the momentary excitation of higher eigenmodes in dynamic atomic force microscopy. Physical Review Letters, 2009. 102(6).

36. Balantekin, M., A.G. Onaran, and F.L. Degertekin, Quantitative mechanical characterization of materials at the nanoscale through direct measurement of time-resolved tip-sample interaction forces. Nanotechnology, 2008. 19(8). 
37. Garcia, R. and E.T. Herruzo, The emergence of multifrequency force microscopy. Nature Nanotechnology, 2012. 7(4): p. 217-226.

38. Sahin, O., Harnessing bifurcations in tapping-mode atomic force microscopy to calibrate time-varying tip-sample force measurements. Review of Scientific Instruments, 2007. 78(10).

39. Sahin, O., Time-varying tip-sample force measurements and steady-state dynamics in tapping-mode atomic force microscopy. Physical Review B, 2008. 77(11).

40. Sahin, O. and N. Erina, High-resolution and large dynamic range nanomechanical mapping in tapping-mode atomic force microscopy. Nanotechnology, 2008. 19(44).

41. Sahin, O., S. Magonov, C. Su, C.F. Quate, and O. Solgaard, An atomic force microscope tip designed to measure time-varying nanomechanical forces. Nature Nanotechnology, 2007. 2(8): p. 507-514.

42. Sarioglu, A.F., S. Magonov, and O. Solgaard, Tapping-mode force spectroscopy using cantilevers with interferometric high-bandwidth force sensors. Applied Physics Letters, 2012. 100(5).

43. Sarioglu, A.F. and O. Solgaard, Cantilevers with integrated sensor for timeresolved force measurement in tapping-mode atomic force microscopy. Applied Physics Letters, 2008. 93(2).

44. Sarioglu, A.F. and O. Solgaard, Modeling, design, and analysis of interferometric cantilevers for time-resolved force measurements in tapping-mode atomic force microscopy. Journal of Applied Physics, 2011. 109(6). 


\section{Investigation of temperature-induced mechanical changes in supported bilayers by variants of tapping mode atomic force microscopy ${ }^{1}$}

Tapping mode atomic force microscopy (AFM) is an invaluable technique for examining topographical features of biological materials in solution, but there has been a growing interest in developing techniques to provide further compositional contrast and information concerning surface material properties. Phase shifts, cantilever response at higher harmonic frequencies of the drive, and time-resolved tip/sample force reconstruction have all been shown to provide additional compositional contrast of surfaces, as a compliment to the tapping mode AFM imaging process. This study demonstrates the relative ability of these different imaging techniques to detect temperature induced changes in the elastic modulus of supported total brain lipid extract (TBLE) bilayer patches on mica. To aid in direct comparison between the different imaging techniques, all required data was obtained simultaneously while capturing traditional tapping mode AFM topographic images. While all of the techniques were able to provide compositional contrast consistent with known temperature-induced changes in the bilayer patch, interpretation of the resulting contrast was not always straightforward. Phase imaging suffered from contrast inversion, and individual harmonics responded in a variety of ways to the temperature-induced changes in elastic modulus of the bilayer. The maximum tapping force (or largest positive force) associated with imaging the bilayer correctly reflected the changes in elastic modulus of the lipid bilayer. Importantly, as the required data can be obtained simultaneously, combining these different imaging techniques can lead to a more complete understanding of a sample's mechanical features.

\subsection{Introduction}

There has been a considerable effort to develop techniques based on tapping mode atomic force microscopy (TMAFM) that simultaneously determine mechanical properties of surfaces while obtaining traditional topographic images. These approaches offer several potential advantages over other traditional AFM techniques that measure material properties, such as high spatial resolution and relatively nondestructive imaging forces

\footnotetext{
${ }^{1}$ This chapter is based on the article, "Investigation of Temperature-Induced Mechanical Changes in Supported Bilayers by Variants of Tapping Mode Atomic Force Microscopy" in Scanning.
} 
that are associated with TMAFM. Other techniques that measure material properties of sample surfaces, such as force volume imaging and nano-indentation, can cause large deformations of soft samples which may result in sample damage. Accordingly, TMAFM-based techniques are particularly well-suited for studying delicate biological specimens in solution.

In the past few years, several TMAFM-based techniques have been developed that simultaneously capture topographical and material information of surfaces. Phase contrast imaging was one of the first such methods, which is based on measuring the phase shift between the drive signal and the cantilever motion $[1,2]$. One can also gain further insight into the material properties of a sample surface by examining the cantilever deflection trajectory at higher harmonic frequencies with respect to the drive frequency $[3,4]$. By applying a harmonic drive to the cantilever, the resulting harmonic oscillation of the cantilever is distorted by the tip/sample tapping event during each oscillation cycle [3-6]. This causes a shift in power to the higher harmonic frequencies, and mapping the cantilever response at those higher frequencies can provide additional compositional contrast of surfaces when imaging in liquids [6, 7].

Recently, tip/sample force reconstruction techniques based on TMAFM have gained considerable attention as a method of gaining insight into the material properties of a sample surface. The first force reconstruction technique was developed by Stark et al., where the cantilever deflection trajectory was captured during the TMAFM imaging process, and then converted into a time-resolved tip/sample force by taking the inverse Fourier transform of the Fourier-transformed cantilever deflection trajectory divided by its transfer function [8]. Since then, several other force reconstruction techniques have been developed to obtain the time-resolved tip/sample forces associated with TMAFM [5, 9-13]. One such method was scanning probe acceleration microscopy (SPAM), which utilizes the second derivative of the deflection signal to recover the tip acceleration trajectory that can then be converted into the time-resolved tip/sample force, as thoroughly discussed in previous chapters [5].

TMAFM-based experiments are particularly well-suited for imaging biologically relevant samples in solution due to the nondestructive nature of the tapping event. Lipid bilayers have been used extensively in TMAFM experiments [14-18]; they serve as an 
excellent model of cellular membranes, which are essential components of all living systems. Cell membranes are two-dimensional liquid-crystalline structures that are composed of a variety of amphiphilic lipids [19, 20]. The material properties of cellular membranes are critical to proper cellular functioning, as membranes must have a high degree of lateral fluidity while maintaining structural integrity. The material properties of lipid membranes (such as fluidity, Young's modulus, and adhesion) depend on the lipid composition and on local environmental properties such as $\mathrm{pH}$, pressure, hydration, and temperature [21, 22]. Lipid membranes are predominantly in a fluid phase under physiological conditions; however, thermal fluctuations can alter the phase state of membranes. For example, increasing temperature generally causes lipid membranes to enter a more disordered state, whereas lowering the temperature encourages a more ordered, and potentially nonfluid state [23]. Phase transitions occur at a variety of temperatures for different lipid systems based on the packing efficiency of specific lipid components [20], and these changes in lipid organization can alter cellular functions, such as signal transduction and membrane trafficking [20, 24]. Herein, phase imaging, higher harmonic imaging, and SPAM were evaluated on their ability to track temperature-induced changes and phase transitions of supported lipid bilayers. Importantly, all of these images (from phase, harmonic and SPAM imaging processes) were obtained simultaneously, which allowed for direct comparisons between individual TMAFM experiments.

\subsection{Methodology}

\subsubsection{Sample preparation}

Supported lipid bilayers were prepared in a similar manner as described in Chapter 3. Total brain lipid extract (TBLE, Avanti Polar Lipids) and cholesterol were dissolved in chloroform (ACROS Organics) to a concentration of 70\% TBLE and 30\% cholesterol by mass. The chloroform was removed using a Vacufuge (Eppendorf), and the resulting lipid films were resuspended in phosphate buffered saline (PBS, $\mathrm{pH}$ 7.3) at a concentration of $1 \mathrm{mg} / \mathrm{mL}$. Five sequential freeze-thaw cycles in liquid nitrogen were performed to promote bilayer formation. The lipid suspensions were then placed in a bath sonicator for $20 \mathrm{~min}$ to promote vesicle formation. The resultant vesicle solution was then diluted 50 
times. $5 \mu \mathrm{L}$ of this diluted solution was directly injected into the AFM fluid cell, which already contained $35 \mu \mathrm{L}$ of PBS, and onto a freshly cleaved mica substrate. Supported lipid bilayer patches formed on the mica surface within a few minutes.

\subsubsection{AFM imaging conditions}

In situ AFM experiments were performed with a Nanoscope $\mathrm{V}$ Multimode scanning probe microscope (Veeco, Santa Barbara, CA) using a fluid cell equipped with an O-ring and a V-shaped oxide-sharpened silicon nitride cantilever (Budget Sensors). All topography images were acquired in the tapping mode with acoustic excitation of the cantilever. While the nominal spring constant of the cantilevers provided by the manufacturer was $0.27 \mathrm{~N} / \mathrm{m}$, the actual spring constant of the cantilevers used in this study were calculated using a thermal tuning method, as previously described [25]. All images were acquired using a closed-loop "vertical engage" J-scanner. The scan rate was $1.95 \mathrm{~Hz}$ with cantilever drive frequencies ranging from approximately 8 to $9 \mathrm{kHz}$. The free amplitude of the cantilever was $29.4 \mathrm{~nm}$ for all experiments presented here, and the set-point ratio, defined as the tapping amplitude to the free amplitude, was maintained at 0.8 . Images were captured at $5 \times 1.25 \mu \mathrm{m}$ and $512 \times 128$ pixel resolutions. Topography and phase images were captured using Nanoscope software supplied by the manufacturer. The temperature was increased from $28-37{ }^{\circ} \mathrm{C}$ using the Bio-Heater accessory for the multimode AFM (Veeco, Santa Barbara, CA) and additionally monitored with a thermister incorporated into the fluid cell.

\subsubsection{Higher harmonic imaging and scanning probe acceleration microscopy}

Cantilever deflection trajectories were simultaneously captured during acquisition of each TMAFM image by use of a signal access module (Veeco), a CompuScope 14-Bit A/D Octopus data acquisition card (Gage Applied Technologies, Lachine, QB, Canada), and custom-written software. Trajectories were captured at 2.5 MS/s and 14-bit resolution with a vertical resolution of $\pm 2 \mathrm{~V}$. These cantilever trajectories were processed using Matlab equipped with the signal processing toolbox (Mathworks, Natick, MA). A sliding window Fourier transform was applied to the cantilever deflection trajectory, which was six oscillation cycles in length and was advanced by one oscillation cycle at a time. To 
create higher harmonic images, it was necessary to take the magnitude of the resulting power spectrums based on individual harmonic frequencies, then reshape the resulting response at any given frequency into an appropriately sized matrix corresponding to a TMAFM image. The time-resolved tip/sample forces were reconstructed from the cantilever deflection trajectory based on the SPAM procedure, exactly as described in Chapter 2. Specifically, the maximum tapping force $\left(F_{\max }\right)$, defined as the largest positive force experienced between the tip and the surface during one tapping event, was organized into an appropriately sized matrix corresponding to the TMAFM image to obtain a spatially resolved force map.

\subsection{Results and discussion}

\subsubsection{Influence of temperature on bilayer height and phase}

To determine the relative applicability of phase imaging, higher harmonic imaging, and reconstruction of the time-resolved tapping forces to study temperature-induced mechanical changes in biologically relevant samples, TMAFM was performed on lipid bilayer patches supported on a mica surface. Lipid bilayers have a considerably smaller elastic modulus compared to mica; therefore, the bilayer patch on mica is an excellent system for comparing different imaging modes because the system provides a surface with a soft region (the supported bilayer patch) and a hard region (mica). The supported lipid bilayer patch was imaged as temperature was increased from 28 to $37^{\circ} \mathrm{C}$, which was accomplished by sequential heating of the sample. Importantly, all of the data (topographic, phase, harmonics, and the maximum tapping forces) was obtained simultaneously during the TMAFM imaging process. Although this experiment took over 8 hours to complete and it was repeated several times, it is important to note that the data was collected from the same bilayer patch using a single cantilever with a constant drive amplitude, free amplitude, and set-point ratio. To accomplish the maintenance of these settings, it was critical that there were no adjustments made to the cantilever placement within the fluid cell for the duration of the experiment. This normalized errors associated with the geometry of the fluid cell and the position of the piezo shaker with respect to the cantilever, so that direct comparisons could be made from image to image as the temperature was adjusted during a single experiment. By maintaining these parameters, 
the total tip/sample force per oscillation cycle $\left(F_{\text {total }}\right)$ remained constant, based on the following equation:

$$
F_{\text {total }} \approx 0.5 k a_{0} \frac{\Delta A}{A_{0}}
$$

where $k$ is the spring constant, $a_{0}$ is the drive amplitude, $\Delta A$ is the tapping amplitude and $A_{0}$ is the free amplitude [26]. As previously described, the experiments in this work were performed using a set-point ratio of 0.8. This value was chosen based on previous studies demonstrating that this setting was appropriate for imaging supported TBLE bilayer patches $[16,27]$. Additionally, it was critical to use only patches of TBLE bilayers rather than a fully formed bilayer, so that the exposed mica surface could serve as an internal control. This control was used to verify that $F_{\text {total }}$ remained constant and to facilitate direct comparisons between images acquired at various temperatures [27]; therefore, all results are reported relative to this control. Although the absolute values for bilayer height, phase shifts, amplitude response of individual harmonics and tapping forces could have varied from experiment to experiment due to the difficulties in controlling the aforementioned imaging parameters, the relative shifts in these values compared to the internal mica control were consistently reproduced, with the exception of phase images, which will be discussed later. For simplicity, the images presented here were gathered from a single experiment.

Similar to the previous study, total brain lipid extract (TBLE) was chosen because it is a naturally occurring mixture of phospholipids. The lipids in the extract all have varying degrees of saturation, which can contribute to the packing arrangement within the lipid bilayer. For this study, TBLE was enriched with $30 \%$ exogenous cholesterol to strengthen the bilayer patches for the lengthy study, as previous studies on TBLE with 0$50 \%$ exogenous cholesterol demonstrated that TBLE bilayers with $30 \%$ exogenous cholesterol have the greatest degree of vertical rigidity [16]. Additionally, incorporation of exogenous cholesterol into the bilayer lowers the phase transition temperature of lipid bilayers [28, 29], allowing observation of phase transitions at temperatures accessible by our experimental set-up. Significant changes in the mechanical properties of bilayers 

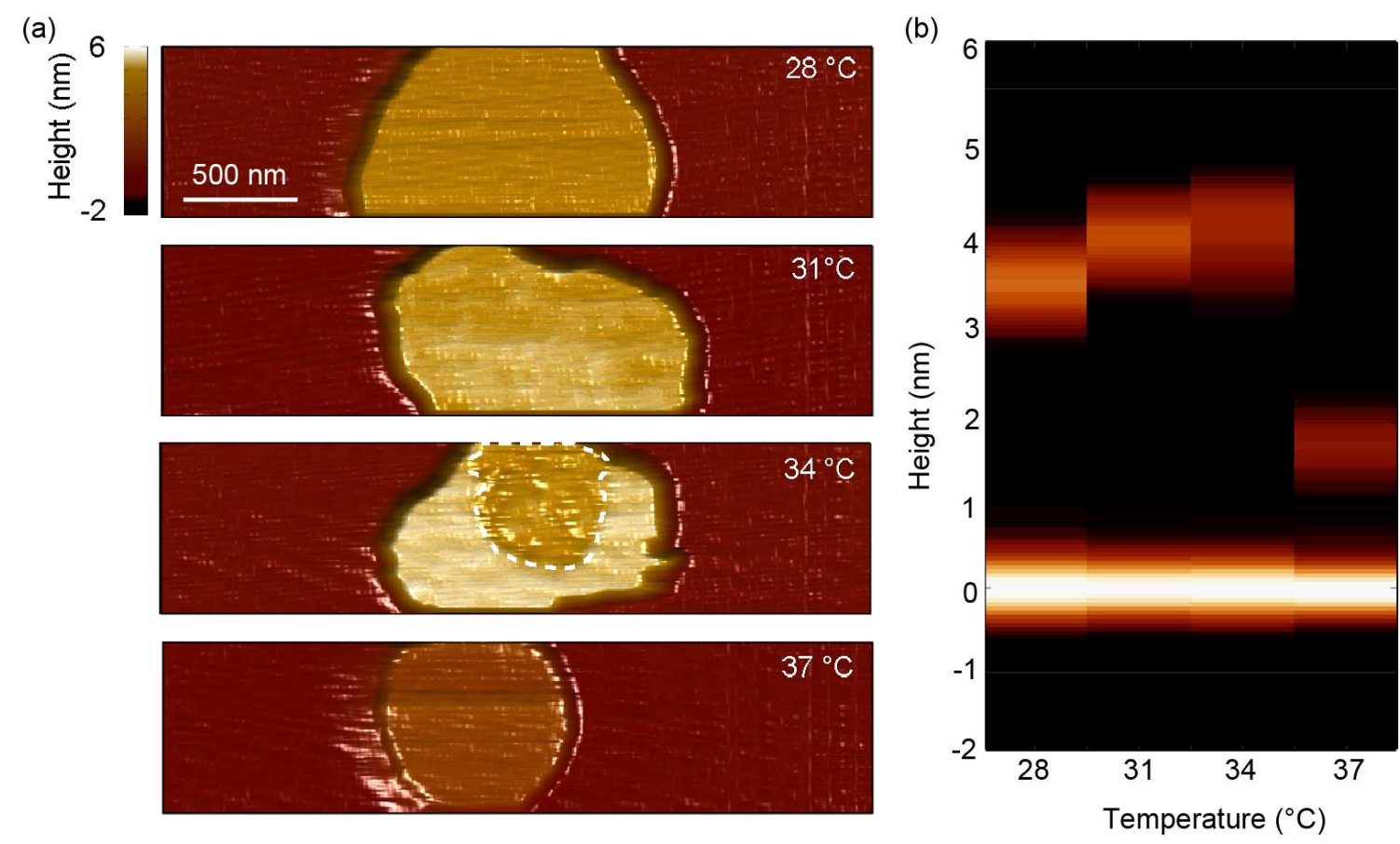

Figure 1. Surface topography of a TBLE bilayer patch supported on mica. (a) AFM topography images of the same patch supported taken at $28,31,34$, and $37^{\circ} \mathrm{C}$. The dotted line in the image taken at $34{ }^{\circ} \mathrm{C}$ indicates a region of the bilayer undergoing a phase transition. The color bar is applicable to all images. (b) Histograms of all of the height measurements in the AFM images presented as a function of temperature. Brighter colors indicate a larger number of measurements corresponding to a specific height. Measurements corresponding to the mica substrate are indicated by the bright region at 0 nm.

accompany phase transitions, thereby providing a simple system to investigate the ability of different TMAFM-based imaging techniques to detect such changes.

To determine if a phase transition occurred in the TBLE bilayer patch upon heating, standard TMAFM topography images were used to evaluate fluctuations in bilayer height as a function of temperature (Fig. 1). At $28{ }^{\circ} \mathrm{C}$, the lipid patch had a height of $3.6 \pm 0.1 \mathrm{~nm}$ (Fig. 1), consistent with previous reports [16, 27]; however, the measured bilayer height was slightly lower than the theoretical height of the lipid bilayers, which is $\sim 4.5-5 \mathrm{~nm}$, due to the compressing action of the cantilever tip on the supported bilayer [27]. This observed compression was consistent with previous TMAFM-based studies and simulations that demonstrated the bilayer is a more compliant surface than the mica substrate [16]. The bilayer height slightly increased $(4.1 \pm 0.5 \mathrm{~nm}$ and $4.2 \pm 0.5 \mathrm{~nm}$ respectively) when the temperature was raised to 31 and $34{ }^{\circ} \mathrm{C}$. 
Furthermore, morphological changes were observed within the bilayer patch at $34{ }^{\circ} \mathrm{C}$, as a region of the bilayer had a height that was $\sim 0.7 \mathrm{~nm}$ smaller (Fig. 1). This indicated that the bilayer was undergoing the initial stages of a transition to a phase that is more easily compressed by the imaging force. Accordingly, the height of the bilayer patch became considerably smaller $(1.8 \pm 0.1 \mathrm{~nm})$ as the temperature was further increased to $37^{\circ} \mathrm{C}$. At lower temperatures, lipid components are more tightly packed within the bilayer, which results in a slightly thicker bilayer; however, the change in thickness of bilayers in this temperature range should only be on the order of a few angstroms [30, 31]. In these studies, the difference in bilayer height before and after the phase transition is much larger than would be expected due to simple rearrangement of lipid packing. This effect is a likely consequence of the cantilever tip pushing deeper into the highly fluid bilayer patch, associated with a phase transition that occurred in the bilayer. This observation is consistent with a previous report demonstrating that changes in the observed height of a bilayer measured by TMAFM in solution can be directly related to changes in the material properties of a lipid bilayer [16]. Unfortunately, there is not a straightforward method to determine the uncompressed height of the bilayer at each temperature, which is required to determine the degree of cantilever indentation into the lipid bilayer patch.

As the temperature of the system was increased from $28^{\circ} \mathrm{C}$ to $37^{\circ} \mathrm{C}$, the shape of the bilayer patch changed considerably (Fig. 1), indicating that the bilayer was quite fluid as expected from the literature $[16,32]$. This effect could have been caused by several factors. First, large, continuous areas of supported lipid bilayers (on the order of 50 by 50 $\mu \mathrm{m}$ or larger) are typically formed by continually scanning an AFM tip across the substrate [14], where the force exerted by the probe pushes the lipid vesicles to the surface, flattening their structure, and promoting vesicle fusion [33]. This experiment, however, was performed at low vesicle concentration to prevent the formation of a continuous bilayer; thus, it is possible that the imaging process actively altered the morphology of the bilayer, which can be quite malleable. Alternatively, the lipid patch occupied the largest observable area at $28{ }^{\circ} \mathrm{C}$, and the size of the patch decreased with increasing temperature. This observation is this is consistent with studies that examined the area of various PC bilayers as a function of temperature [30], where a condensing 
effect occurred in order to maintain structural integrity as the lateral fluidity of membranes increased.

To obtain the topographical images at each temperature during the TMAFM imaging procedure, phase images of the lipid bilayer patches on mica were simultaneously acquired. The phase signal of the cantilever operated in TMAFM can be used to gain insight into the mechanical properties of sample surfaces. For this experiment, the phase images were normalized so that the phase shift associated with tip/sample tapping events on mica had a value of zero. This allowed for direct comparisons between phase images taken at different temperatures (Fig. 2). Distinct contrast was observed between the mica substrate and the bilayer patch at $28{ }^{\circ} \mathrm{C}$, with the phase shifting $2.1 \pm 0.1^{\circ}$ on the bilayer with respect to the mica substrate. The phase shift associated with the bilayer increased in magnitude with a larger distribution of values as
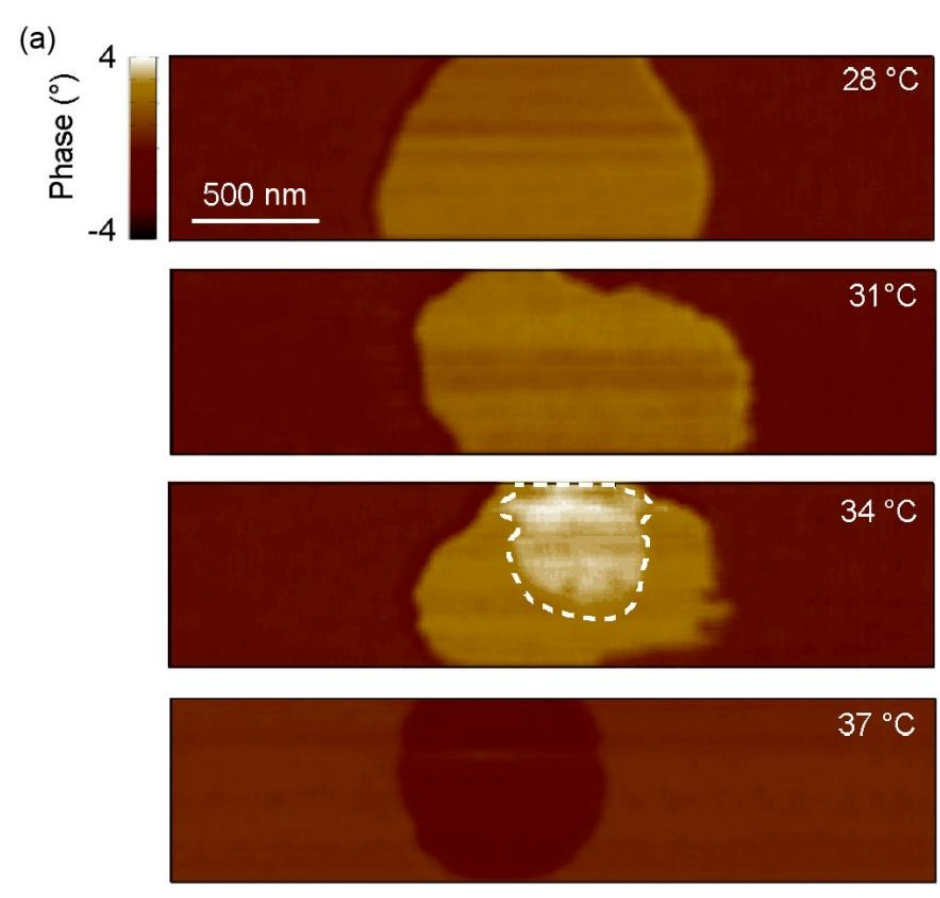

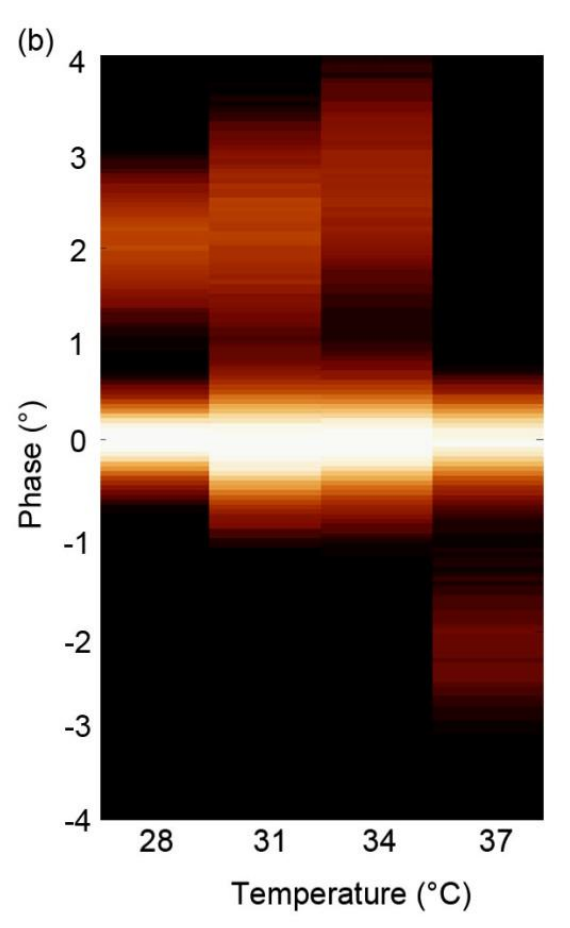

Figure 2. Cantilever phase response to imaging a TBLE bilayer patch supported on mica. (a) AFM phase images of the same bilayer patch taken at 28, 31, 34, and $37{ }^{\circ} \mathrm{C}$. The dotted line in the image taken at $34{ }^{\circ} \mathrm{C}$ indicates a region of the bilayer undergoing a phase transition as determined from the topography image shown in Figure 1. The color bar is applicable to all images. (b) Histograms of all of the phase measurements in the AFM images presented as a function of temperature. Brighter colors indicate a larger number of measurements corresponding to a specific phase shift. 
the temperature was raised to 31 and $34{ }^{\circ} \mathrm{C}\left(2.5 \pm 0.1^{\circ}\right.$ and $3.1 \pm 0.1^{\circ}$, respectively). At 34 ${ }^{\circ} \mathrm{C}$, the region of the bilayer associated with the initial transition to a more fluid state had a clear phase contrast compared with the rest of the bilayer. Additionally, at lower temperatures, the phase shift associated with the bilayer was always positive, but the phase shift associated with the bilayer at $37{ }^{\circ} \mathrm{C}$ was negative $\left(-2.3 \pm 0.1^{\circ}\right)$ with respect to the mica substrate. This inversion of contrast represents one of the ambiguities related to phase imaging, as the phase contrast between regions of differing elastic modulus are not always consistently in one direction [34]. Inversion of contrast at higher temperatures (equal to or greater than $37^{\circ} \mathrm{C}$ ) was not always observed in separate experiments (Fig. 3); however, there was always some appreciable change (on the order of 1-3 degrees) in phase contrast when heating the bilayer above its transition temperature. Despite this ambiguity in phase response as a function of temperature, the phase shifts indicate that the mechanical properties associated with the bilayer do change with the increase in temperature. This supports the rationale that the differences in height are due to a temperature-induced phase transition in the bilayer.

Phase contrast images can provide insight into the material properties of a sample surface, and are typically associated with tip/sample energy dissipation [2, 35, 36]. Although great progress has been made in the theory underlying phase contrast [37], interpretation of phase images remains difficult due to a variety of competing sources of

(a)

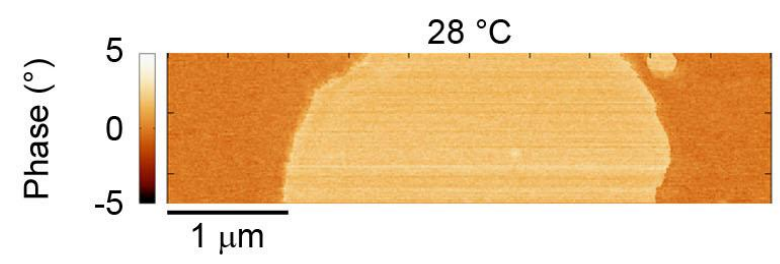

(b)

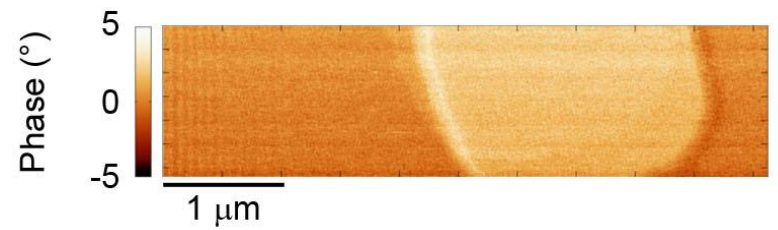

$37^{\circ} \mathrm{C}$
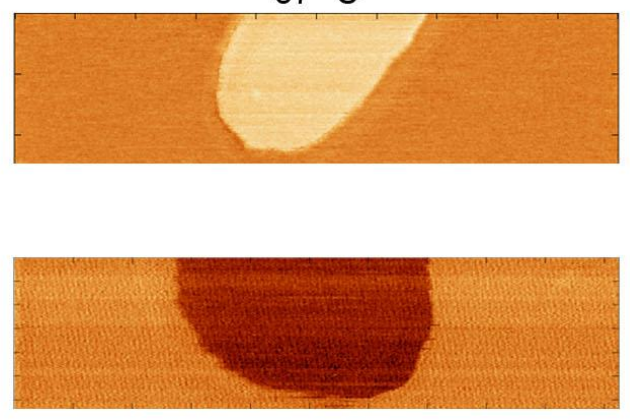

Figure 3. Phase images of TBLE bilayer patches supported on mica from two additional experiments. These images demonstrate that the phase shift associated with the transition in the bilayer was inconsistent. That is, in some replicates (a) no contrast inversion was observed, while (b) contrast inversion was observed in others. Nevertheless, there was always a shift in the phase associated with phase images above $37^{\circ} \mathrm{C}$. 
energy dissipation (i.e., capillary forces [38], viscoelasticity of the sample [39], and cross talk with topography [40]) associated with tip/sample interactions. Phase contrast is also highly dependent on imaging parameters, such as free amplitude and set-point ratio [41, 42]. For example, changing the set-point ratio in TMAFM can even lead to contrast inversion in phase images [34]. Accordingly, using phase imaging to elucidate mechanical properties of surfaces can be complicated without a priori knowledge of those properties, and imaging samples in solution can further complicate these issues [41, 43]. In this study, the chosen imaging conditions were optimized for topographical imaging of the bilayer patch, and not phase contrast imaging. It is possible that imaging conditions could been slightly modified so that the phase contrast images more accurately and reproducibly reflected the relative compressibility of the bilayer patch with respect to mica.

While it would be interesting to correlate changes in phase contrast to the differing ability of the bilayer to dissipate energy before and after undergoing a transition, the relationship between phase contrast and tip/sample energy dissipation is based on the assumption of cantilever dynamics being dominated by a single eigenmode. However, when operating TMAFM in solution using soft cantilevers $(k<1 \mathrm{~N} / \mathrm{m})$ with low quality factors $(Q<5)$, the second eigenmode of the cantilever plays a prominent role [44], and the assumption of a single eigenmode dominating the tip/sample interaction is not met, making it difficult to directly relate phase contrast to tip/sample dissipation alone [37]. Melcher et al. demonstrated that the phase contrast associated with such soft cantilevers can arise from either: (i) energy propagation during the tapping event that excites higher eigenmodes of the cantilever, which is mediated by conservative short-range interactions such as elastic contact forces between the cantilever tip and sample surface, or (ii) tip/sample energy dissipation [37]. In this experimental system, which consists of a soft biological lipid bilayer supported on a comparably stiff mica substrate in a high-ionic strength buffer, the DLVO forces are highly screened and adhesion hysteresis is negligible [37, 45, 46]. As a result the observed inversion of contrast in some phase images of the bilayer on mica at $37{ }^{\circ} \mathrm{C}$ compared to images taken at different temperatures can be attributed to these two sources of phase contrast. At the lower temperatures, the phase contrast is dominated by the momentary propagation of energy 
from the first to the second eigenmode of the cantilever; whereas, the tip/sample dissipative interaction dominates at $37{ }^{\circ} \mathrm{C}$. Melcher et al. also point out that phase images should not be considered maps of elastic modulus, but rather local stiffness [37].

\subsubsection{Observation of compositional contrast using higher harmonic imaging}

The harmonic motion of the cantilever is distorted during the tapping event due to the nonlinear interaction between the tip and sample during tapping mode AFM. As a consequence, higher frequencies that are harmonic to the drive frequency are excited within the cantilever [44]. As a result, compositional contrast can be achieved by monitoring harmonic frequencies, and higher harmonic imaging has been used to observe subtle surface features that are difficult to distinguish by ordinary tapping mode AFM experiments [3]. Higher harmonic imaging is especially useful for imaging in solution, as the large distortion of cantilever motion associated with imaging in liquid leads to a larger excitation of higher harmonics [6].

In this study, AFM images based on the response of specific higher harmonic frequencies were obtained by capturing the entire cantilever deflection trajectory while imaging by TMAFM. Using a sliding window Fourier transform, the cantilever response at specific harmonic frequencies was then obtained and reshaped into an appropriately sized matrix corresponding to the AFM image. This allowed the simultaneous generation of individual harmonic images for many different harmonic signals of the same TBLE bilayer patch, while raising the temperature from $28-37{ }^{\circ} \mathrm{C}$ (Fig. 4-8). The first harmonic (data not shown) is analogous to the standard amplitude or error images associated with TMAFM. Although the amplitude response is sensitive to the edges of the bilayer patch on the mica surface and it provides important information concerning the efficiency of the feedback loop, it contains little information concerning the mechanical properties of the surface. However, higher harmonics have been used to map a variety of surfaces properties [6, 7, 47]. While the results presented here are for one replicate of the experiment, it should be noted that the relative response of the individual harmonics were reproducible from experiment to experiment, i.e., the shift of the harmonic response associated with imaging the bilayer patch at each temperature with respect to the internal standard in mica was the same. However, it was difficult to reproduce exact experimental 

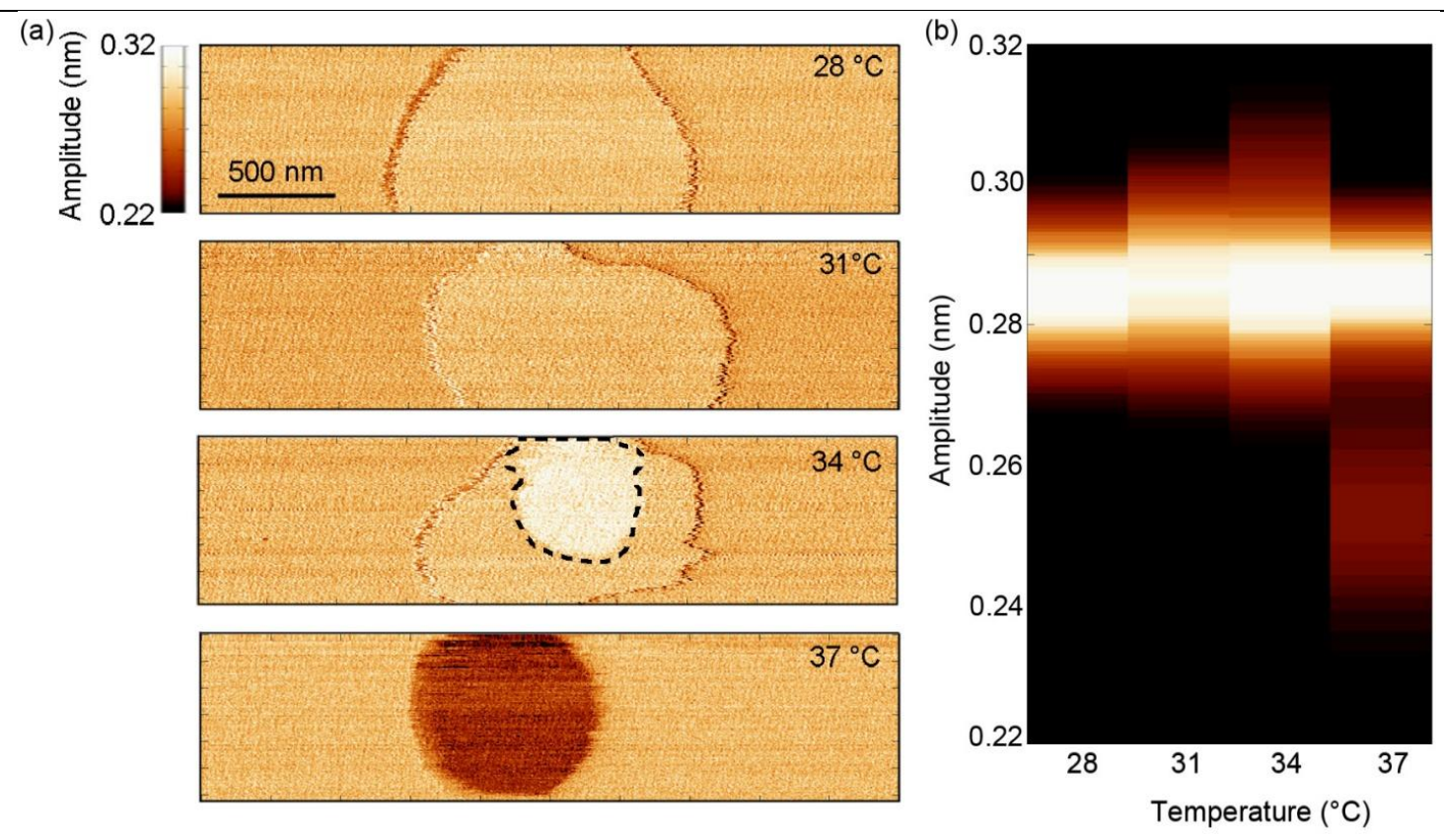

Figure 4. Images and histograms corresponding to the $2^{\text {nd }}$ harmonic. (a) $2^{\text {nd }}$ harmonic AFM images of the same TBLE bilayer patch supported on mica taken at 28, 31, 34, and $37{ }^{\circ} \mathrm{C}$. The dotted line in the image taken at $34{ }^{\circ} \mathrm{C}$ indicates a region of the bilayer undergoing a phase transition as determined from the topography image shown in Figure 1. The color bar is applicable to all images. (b) Histograms of all of the $2^{\text {nd }}$ harmonic responses in the AFM images presented as a function of temperature. Brighter colors indicate a larger number of measurements corresponding to a specific response of the $2^{\text {nd }}$ harmonic. Measurements corresponding to the mica are indicated by the bright region near $0.285 \mathrm{~nm}$.

parameters between each replicate, so the magnitude of the observed amplitudes associated with harmonic in the different replicates varied up to about 10-20\%. Harmonic responses above the first provided contrast between the supported bilayer and mica surface as the temperature increased, and a select few will be discussed for illustrative purposes. For the $2^{\text {nd }}$ harmonic (Fig. 4), contrast between the lipid and the mica in images obtained at $28-34{ }^{\circ} \mathrm{C}$ was quite small, and distinct populations of cantilever response at this frequency were not apparent in the histograms. At these temperatures, the typical response of the second harmonic was on the order of $0.28-0.29 \mathrm{~nm}$; however, the $2^{\text {nd }}$ harmonic showed contrast between the transitioning domain of the bilayer at $34{ }^{\circ} \mathrm{C}$ and the surrounding lipid. Interestingly, after the bilayer went through a phase transition (image taken at $37{ }^{\circ} \mathrm{C}$ ), there was clear contrast in the $2^{\text {nd }}$ harmonic image between the bilayer and mica, where the bilayer was associated with a smaller amplitude response $(0.25 \pm 0.03 \mathrm{~nm})$ compared to the lower temperatures. Images based on the $5^{\text {th }}$ harmonic 

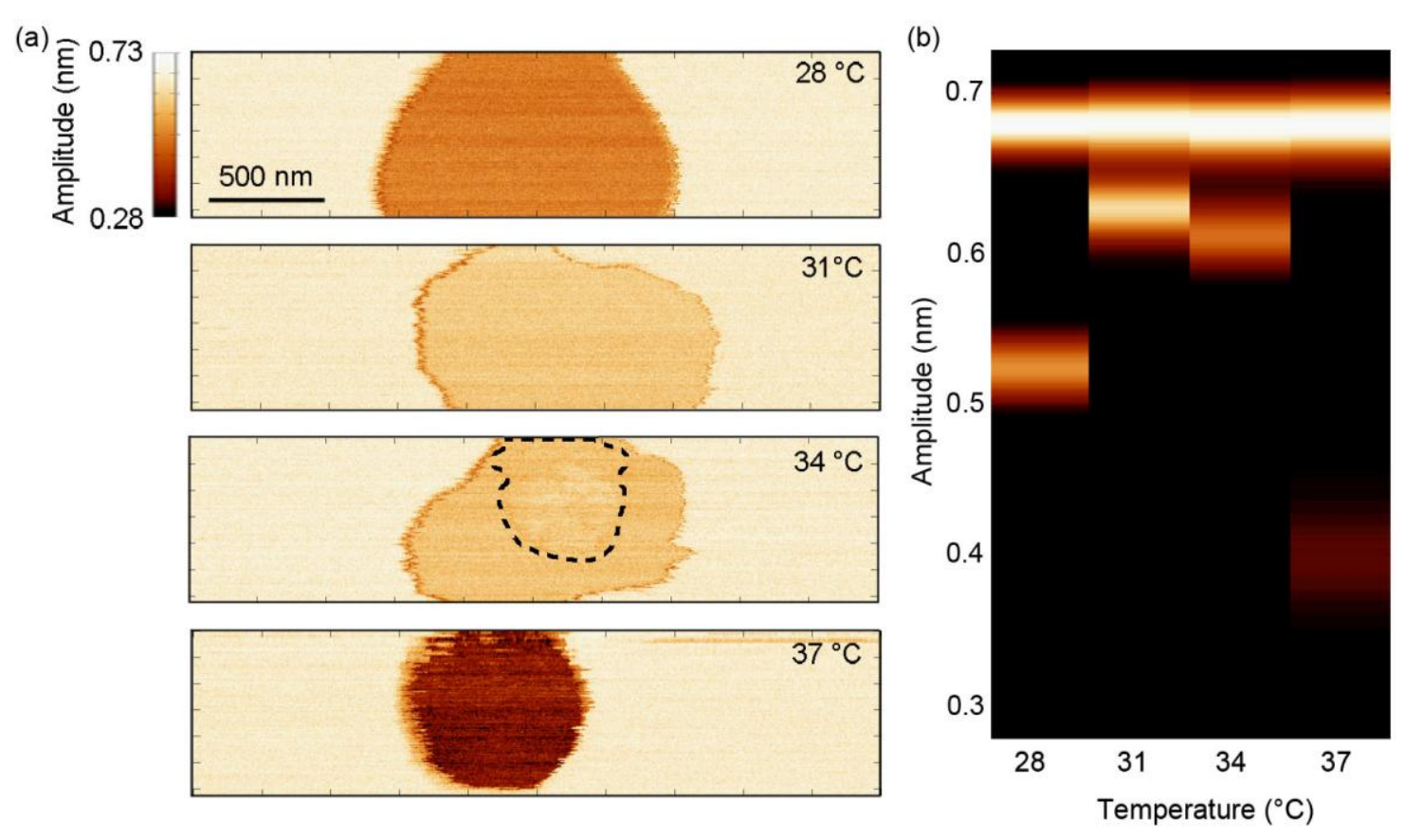

Figure 5. Images and histograms corresponding to the $5^{\text {th }}$ harmonic. (a) $5^{\text {th }}$ harmonic AFM images of the same TBLE bilayer patch supported on mica taken at 28, 31, 34, and $37{ }^{\circ} \mathrm{C}$. The dotted line in the image taken at $34{ }^{\circ} \mathrm{C}$ indicates a region of the bilayer undergoing a phase transition as determined from the topography image shown in Figure 1. The color bar is applicable to all images. (b) Histograms of all of the $5^{\text {th }}$ harmonic responses in the AFM images presented as a function of temperature. Brighter colors indicate a larger number of measurements corresponding to a specific response of the $5^{\text {th }}$ harmonic.

(Fig. 5) provided distinct contrast between the bilayer patch and the mica surface, where the response on the patch always corresponded to a smaller amplitude response. At $28{ }^{\circ} \mathrm{C}$, the response related to the $5^{\text {th }}$ harmonic was $0.52 \pm 0.05 \mathrm{~nm}$, and increased to $0.62 \pm 0.08$ $\mathrm{nm}$ and $0.61 \pm 0.07 \mathrm{~nm}$ at 31 and $34{ }^{\circ} \mathrm{C}$ respectively. However, the $5^{\text {th }}$ harmonic was not particularly sensitive to the region of the bilayer undergoing transition at $34{ }^{\circ} \mathrm{C}$, which resulted in minimal contrast. The response of the $5^{\text {th }}$ harmonic associated with the bilayer shifted to $0.37 \pm 0.02 \mathrm{~nm}$ at $37^{\circ} \mathrm{C}$, which may be indicative of the phase transition in the bilayer. The response of the $5^{\text {th }}$ harmonic was always consistent with the softer bilayer regions being associated with a lower amplitude response compared to the mica, and the magnitude of the response was approximately doubled compared to the $2^{\text {nd }}$ harmonic. Imaging on the $6^{\text {th }}$ harmonic (Fig. 6) resulted in negligible contrast from $28-34{ }^{\circ} \mathrm{C}$, as only the edges of the bilayer patch were distinguishable from the mica; however, there was stark contrast between the bilayer and mica at $37{ }^{\circ} \mathrm{C}$ associated with the $6^{\text {th }}$ harmonic 
(a)

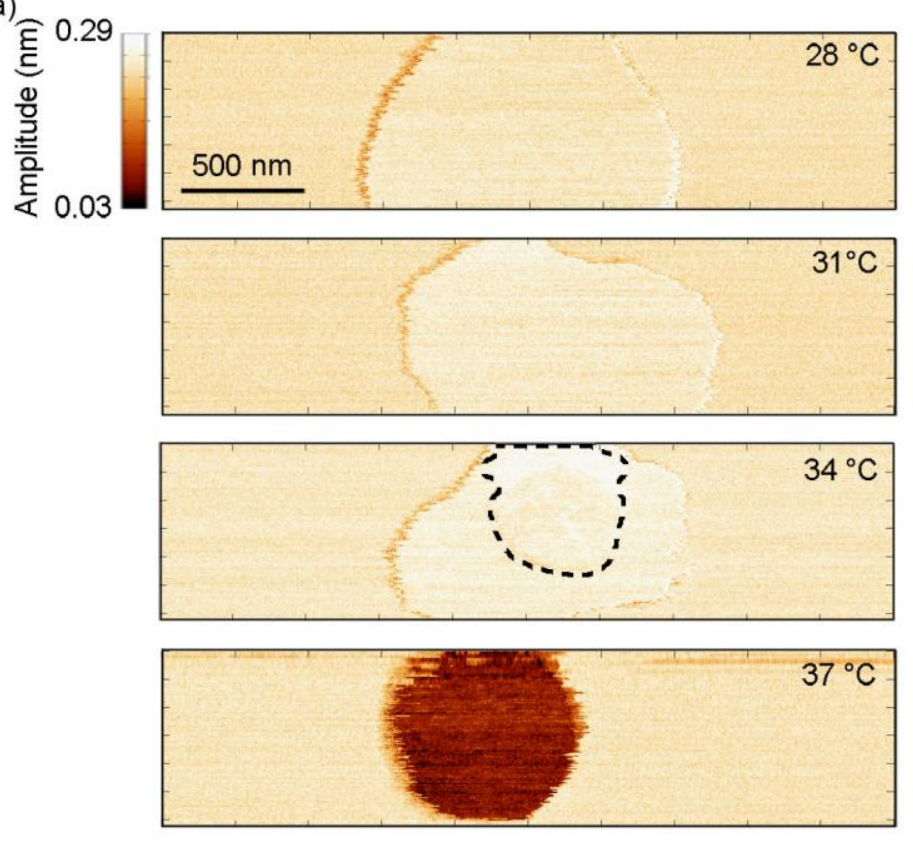

(b)

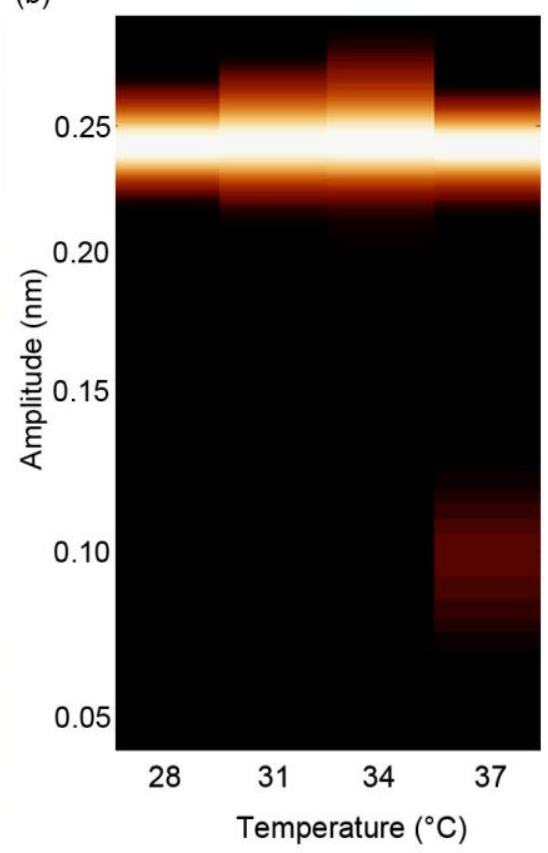

Figure 6 . Images and histograms corresponding to the $6^{\text {th }}$ harmonic. (a) $6^{\text {th }}$ harmonic AFM images of the same TBLE bilayer patch supported on mica taken at 28, 31, 34, and $37{ }^{\circ} \mathrm{C}$. The dotted line in the image taken at $34{ }^{\circ} \mathrm{C}$ indicates a region of the bilayer undergoing a phase transition as determined from the topography image shown in Figure 1. The color bar is applicable to all images. (b) Histograms of all of the $6^{\text {th }}$ harmonic responses in the AFM images presented as a function of temperature. Brighter colors indicate a larger number of measurements corresponding to a specific response of the $6^{\text {th }}$ harmonic.

The magnitude of the $6^{\text {th }}$ harmonic was also reduced to less than $0.26 \mathrm{~nm}$. Images based on the response of the $7^{\text {th }}$ harmonic (Fig. 7) provided distinct contrast between the bilayer and mica at all temperatures. Unlike other harmonic images previously discussed, the amplitude response of the $7^{\text {th }}$ harmonic was larger for the bilayer compared to mica at 28$34{ }^{\circ} \mathrm{C}$. However, the amplitude response of the $7^{\text {th }}$ harmonic associated with the bilayer shifted below that of mica at $37{ }^{\circ} \mathrm{C}$, resulting in an inversion of contrast in the $7^{\text {th }}$ harmonic images as the temperature was raised. Images were also produced for higher harmonics (for example the $10^{\text {th }}$ and $15^{\text {th }}$ harmonics shown in Fig. 8) with varying patterns of contrast as a function of temperature, but the magnitude of the response became quite small ( $<0.15$ and $0.03 \mathrm{~nm}$, respectively). Furthermore, the pattern of cantilever response as a function of temperature was very similar for harmonics ranging from the 12 to the $22^{\text {nd }}$ harmonic (data not shown). 


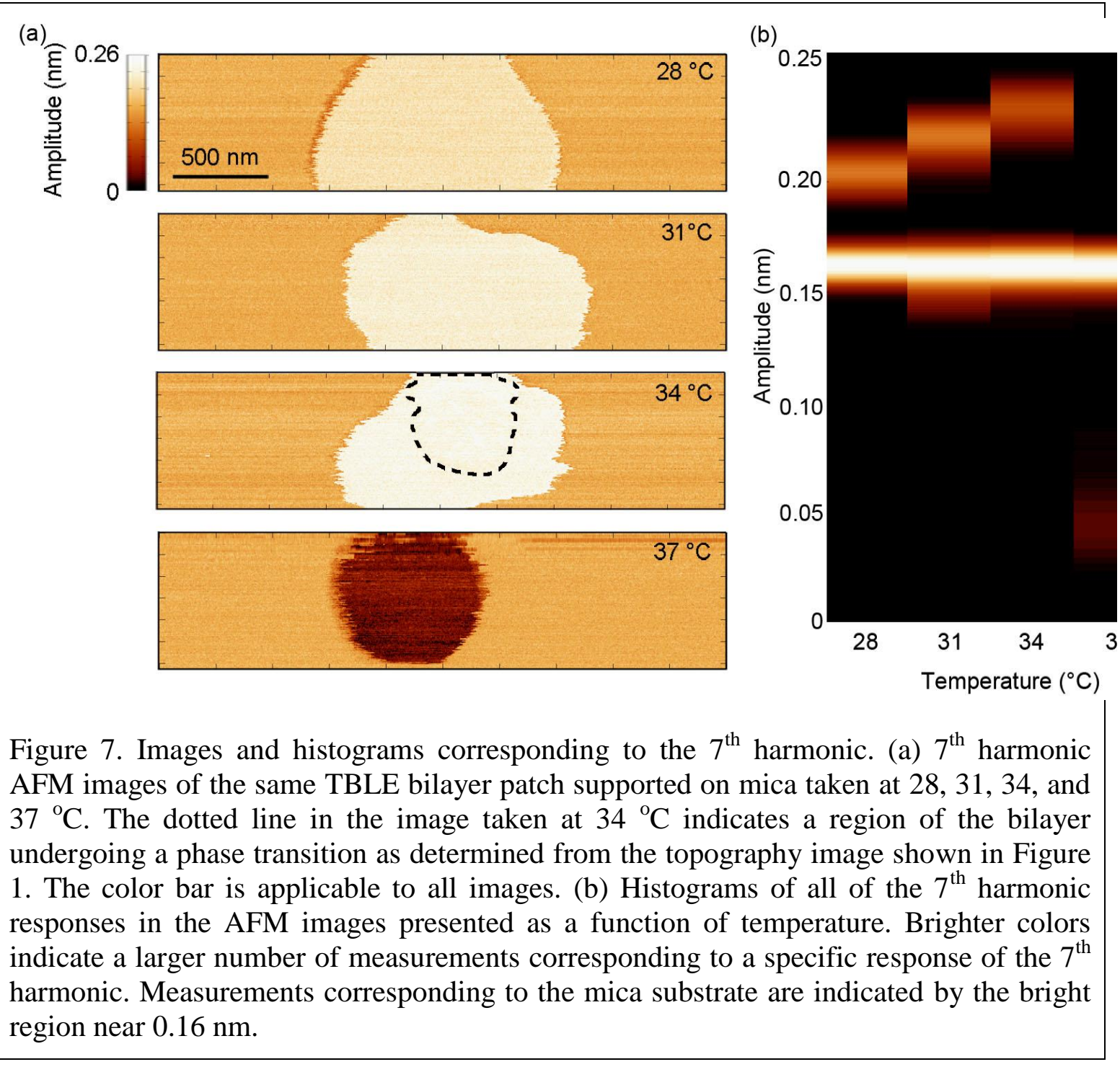

Collectively, obtained images demonstrate that higher harmonics can provide compositional contrast to varying degrees. However, unambiguous interpretation of the contrast afforded by higher harmonics is difficult. While the $2^{\text {nd }}$ harmonic has been used to obtain compositional contrast of soft biological samples imaged in solution [6], it has been found that harmonics near the second eigenmode of the cantilever give larger responses and better contrast [7]. During the tapping event, the second eigenmode is momentarily excited, and this temporarily excites higher harmonics near this natural frequency, and these harmonics have been shown to be highly sensitive to local mechanical properties of the surface [7]. For the cantilever used in this study, the natural frequency of the second eigenmode was nearest to the $5^{\text {th }}$ harmonic, resulting in the larger 


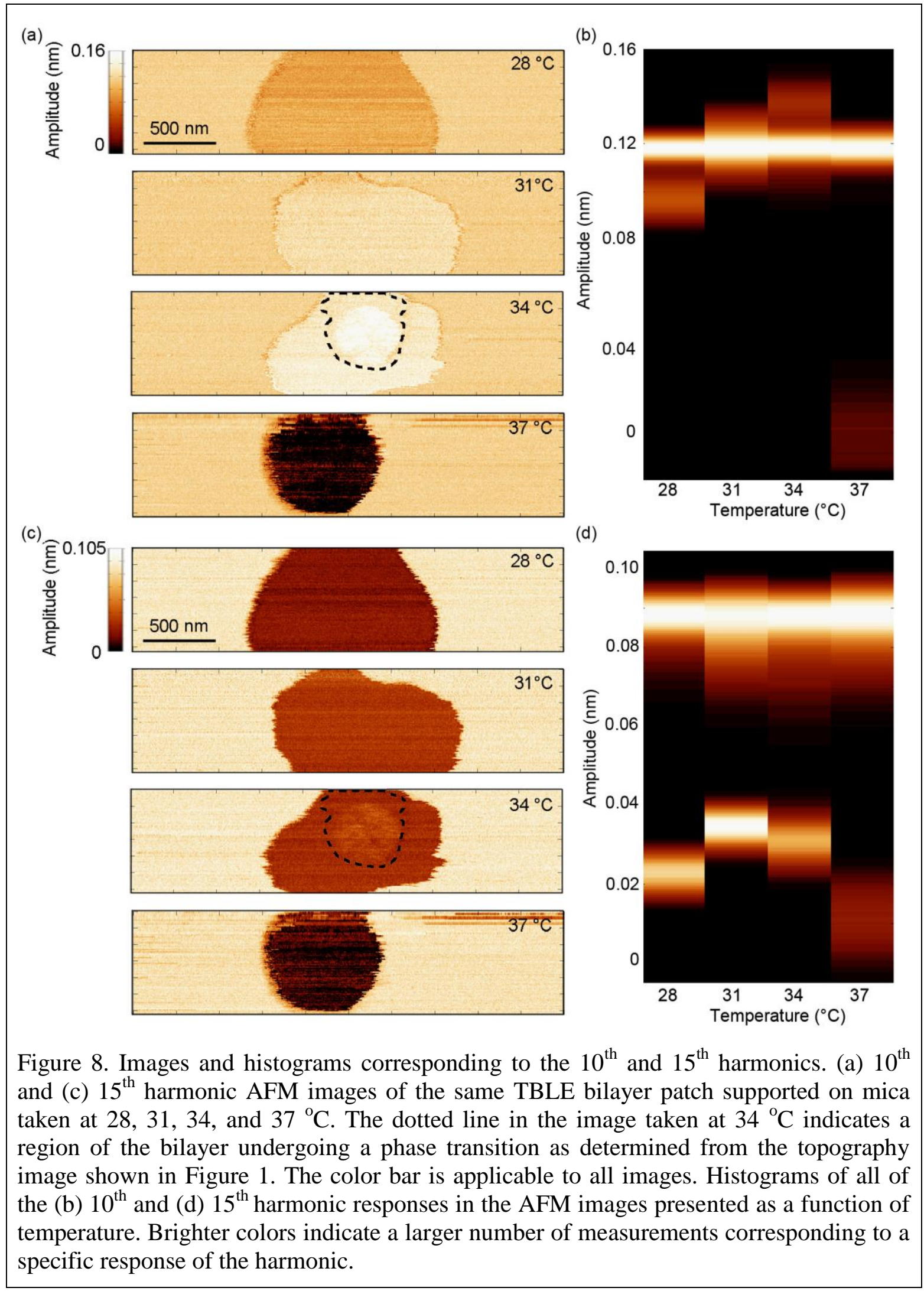


response associated with this harmonic. The higher sensitivity induced by proximity to the second eigenmode explains why the $5^{\text {th }}$ harmonic response closely tracks the known temperature-induced changes in the lipid bilayer patch. Similar to origins of phase contrast with very soft and highly damped cantilevers [37], the contrast of the $5^{\text {th }}$ harmonic is most likely derived from local elastic stiffness of the sample. In general, however, a single harmonic only provides partial information concerning the tip/sample interaction, but methods, such as force reconstruction, that combine the information stored in each individual harmonic may afford a more complete picture of the mechanical properties of samples.

\subsubsection{Maximum tapping forces reflect temperature-induced mechanical changes in the bilayer}

Multiple numerical models and experiments have demonstrated that the maximum force $\left(F_{\max }\right)$, defined as the largest positive force experienced between the tip and surface during the tapping event, increases with increasing elastic modulus of the sample [16, 48, 49]. While this dependence is nonlinear and resembles a power law [49], it can be used to obtain contrast within an image based on relative elastic modulus [14, 50-52]. Although the magnitude of the tapping force is influenced by imaging parameters, the relative value of $F_{\max }$ on the elastic modulus of the surface appears to be maintained. For example, increasing the spring constant of the cantilever results in a larger magnitude of imaging force, but the power law dependence of $F_{\max }$ with the elastic modulus is preserved [53, 54]. Additionally, the relative magnitude of $F_{\max }$ is also preserved with changes in the setpoint ratio and cantilever free amplitude $[16,27]$. To evaluate the ability of $F_{\max }$ images to detect temperature-induced mechanical changes in lipid bilayers associated with a phase transition, SPAM was used to convert the cantilever deflection signal into timeresolved tip/sample forces. The value of $F_{\max }$ associated with every tapping event was used to construct force images of the bilayer patch supported on mica at different temperatures (Fig. 9). As stated earlier, the mica surface was used as an internal reference point to compare $F_{\max }$ values at different temperatures. With this in mind, the relative magnitudes of $F_{\max }$ associated with imaging the bilayer patch with respect to the mica surface was reproduced from replicate to replicate (i.e., the magnitude of $F_{\max }$ associated 
with imaging the bilayer patch was always smaller compared to the internal reference of mica, and there was always a pronounced shift to smaller values of $F_{\max }$ associated with imaging the bilayer after the temperature-induced phase transition).

At all temperatures, the $F_{\max }$ associated with the lipid bilayer was always smaller than the $F_{\max }$ associated with the bare mica substrate, as would be expected based on the relative rigidities of the surfaces (this effect was demonstrated in simulations and experiments of Chapters 2 and 3, respectively) [16]. From $28-34{ }^{\circ} \mathrm{C}$, a slight increase in $F_{\max }(12.4 \pm 0.2 \mathrm{nN}$ to $16.9 \pm 0.2 \mathrm{nN})$, which indicated an initial increase in bilayer rigidity with rising temperatures, was observed. This result is consistent with the observed increase in bilayer height and the notion that increasing temperature raises lateral fluidity within bilayers, as previous studies suggested that higher fluidity
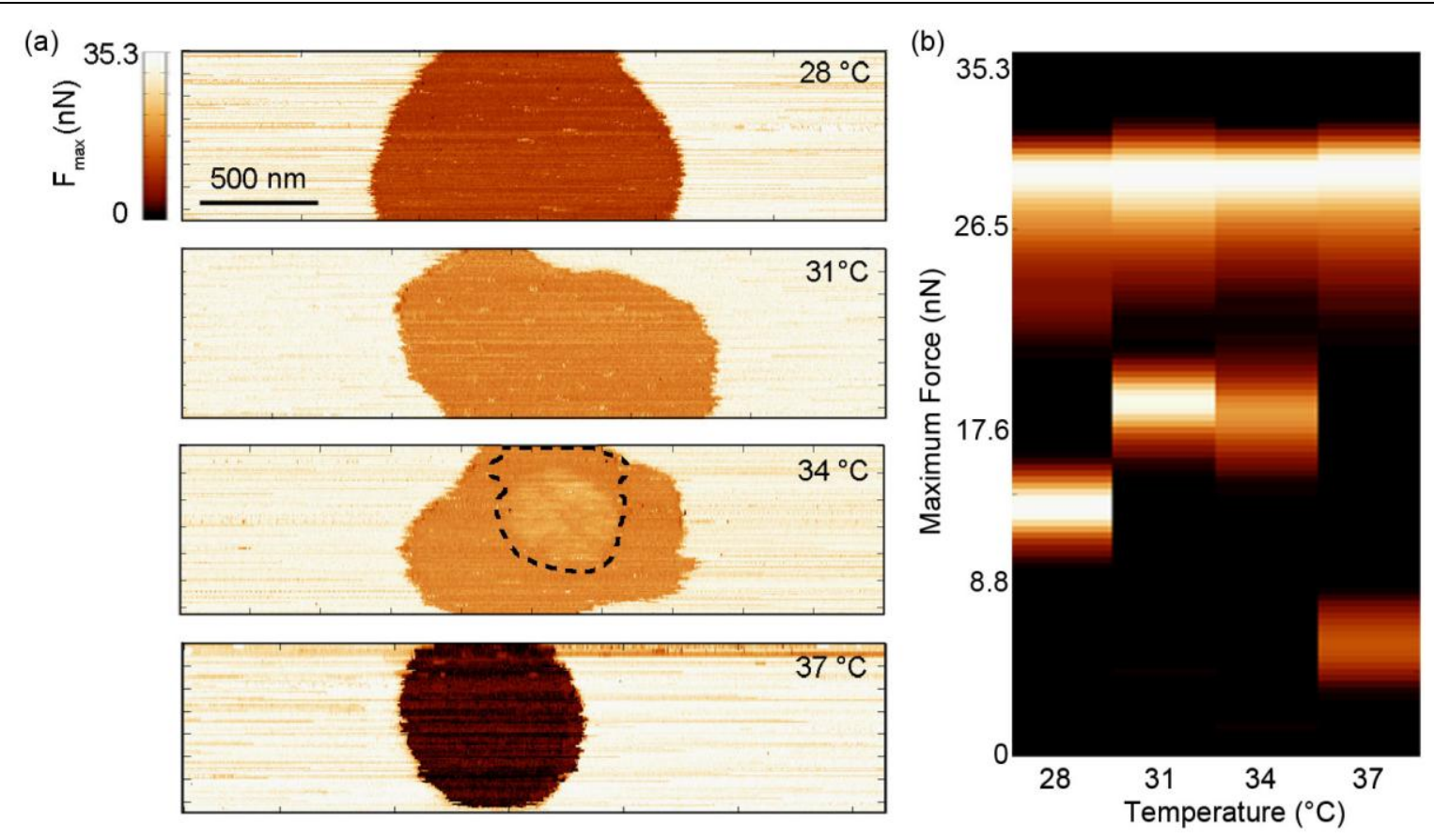

Figure 9. Maximum tapping forces $\left(F_{\max }\right)$ associated with imaging a TBLE bilayer patch supported on mica. (a) $F_{\max }$ AFM images obtained from SPAM of the same bilayer patch taken at $28,31,34$, and $37{ }^{\circ} \mathrm{C}$. The dotted line in the image taken at $34{ }^{\circ} \mathrm{C}$ indicates a region of the bilayer undergoing a phase transition as determined from the topography image shown in Figure 1. The color bar is applicable to all images. (b) Histograms of all of the $F_{\max }$ measurements in the AFM images presented as a function of temperature. Brighter colors indicate a larger number of measurements corresponding to a specific $F_{\max }$. Measurements corresponding to the mica substrate are indicated by the bright region near $28 \mathrm{nN}$. 
corresponds with increased vertical rigidity in bilayers $[16,32]$. At $34{ }^{\circ} \mathrm{C}$, faint contrast was observed for the region of the bilayer that began to transition to a more fluid phase, and this region appeared to be slightly more rigid (higher $F_{\max }$ ). Once the bilayer had undergone the apparent phase transition at $37^{\circ} \mathrm{C}, F_{\max }$ significantly declined $(5.7 \pm 0.1$ $\mathrm{nN})$. The decline of $F_{\max }$ at this higher temperature corresponds with the previously noted decrease in height, supporting the rationale that the bilayer's phase transition resulted in a significantly lower elastic modulus. This decreased rigidity of the bilayer caused the cantilever tip to push deeper into the bilayer surface, thereby compressing the bilayer and resulting in a smaller measured height, consistent with numerical simulations [16]. Importantly, the relative values of $F_{\max }$ were always predictable and in accordance with the known rigidities of the bilayer and mica substrate.

\subsection{Conclusions}

TMAFM is widely used to obtain images with nanoscale spatial resolution of soft samples in liquid, but there has been considerable interest in developing TMAFM-based methods that simultaneously provide compositional contrast and mechanical information. Here, we demonstrated the relative ability of phase, higher harmonics, and force reconstruction (SPAM) imaging to detect changes in the elastic modulus of a lipid bilayer patch supported on mica caused by a temperature-induced phase transition. We made

direct comparisons between these imaging techniques by simultaneously acquiring the different data sets, ensuring that all images were acquired under the same experimental conditions. Using a priori knowledge concerning the sample and height images, we determined which imaging techniques were able to unambiguously reflect the temperature-induced mechanical change in the bilayer. The key mechanical change observed in the supported lipid bilayer was due to a phase transition induced between 34$37{ }^{\circ} \mathrm{C}$, which resulted in a highly compressible bilayer as confirmed by topographic images.

While all of the techniques were able to provide compositional contrast associated with this phase transition, interpretation of this contrast was not always straightforward. Phase imaging suffered from contrast inversion between the known hard and soft regions of the surface in some replicates if the experiment. While a widely used technique for 
mapping mechanical differences on a surface, phase imaging suffers from the high dependence on the phase response to imaging parameters. Individual harmonic images exhibited a variety of response patterns at the different temperatures, with the largest cantilever response being associated with harmonic frequencies near an eigenmode of the cantilever, which for this particular experiment was the $5^{\text {th }}$ harmonic. The response of the

$5^{\text {th }}$ harmonic, of all of the monitored harmonic frequencies, most accurately reflected the known temperature-induced change in bilayer mechanics, suggesting that harmonics near an eigenmode are particularly sensitive to changes in local surface properties. Force reconstruction was accomplished using SPAM, and changes in the $F_{\max }$ (or largest positive force between the tip and the sample) associated with imaging the bilayer correctly reflected the changes in elastic modulus of the lipid bilayer and was able to contrast subtle mechanical changes within the bilayer. This study was aided by the incorporation of an internal standard (exposed mica) so that relative changes in the response of these different imaging modes could be evaluated. Importantly, as the required data can be obtained simultaneously, combining these different imaging techniques can aid in ascertaining a more complete understanding of a sample's mechanical features.

\subsection{References}

1. Anczykowski, B., B. Gotsmann, H. Fuchs, J.P. Cleveland, and V.B. Elings, How to measure energy dissipation in dynamic mode atomic force microscopy. Applied Surface Science, 1999. 140(3-4): p. 376-382.

2. Cleveland, J.P., B. Anczykowski, A.E. Schmid, and V.B. Elings, Energy dissipation in tapping-mode atomic force microscopy. Applied Physics Letters, 1998. 72(20): p. 2613-2615.

3. Stark, R.W. and W.M. Hecki, Higher harmonics imaging in tapping-mode atomic-force microscopy. Review of Scientific Instruments, 2003. 74(12): p. 5111.

4. Hillenbrand, R., M. Stark, and R. Guckenberger, Higher-harmonics generation in tapping-mode atomic-force microscopy: Insights into the tip-sample interaction. Applied Physics Letters, 2000. 76(23): p. 3478-3480. 
5. Legleiter, J., M. Park, B. Cusick, and T. Kowalewski, Scanning probe acceleration microscopy (SPAM) in fluids: mapping mechanical properties of surfaces at the nanoscale. Proceedings of the National Academy of Sciences of the United States of America, 2006. 103: p. 4813-4818.

6. Preiner, J., J. Tang, V. Pastushenko, and P. Hinterdorfer, Higher Harmonic Atomic Force Microscopy: Imaging of Biological Membranes in Liquid. Physical Review Letters, 2007. 99(4): p. 046102.

7. Xu, X., J. Melcher, S. Basak, R. Reifenberger, and A. Raman, Compositional Contrast of Biological Materials in Liquids Using the Momentary Excitation of Higher Eigenmodes in Dynamic Atomic Force Microscopy. Physical Review Letters, 2009. 102(6): p. 060801.

8. Stark, M., R.W. Stark, W.M. Heckl, and R. Guckenberger, Inverting dynamic force microscopy: From signals to time-resolved interaction forces. Proceedings of the National Academy of Sciences of the United States of America, 2002. 99(13): p. 8473-8478.

9. Dong, M., S. Husale, and O. Sahin, Determination of protein structural flexibility by microsecond force spectroscopy. Nature Nanotechnology, 2009. 4(8): p. 514517.

10. Sahin, O., G. Yaralioglu, R. Grow, S.F. Zappe, A. Atalar, C. Quate, and O. Solgaard, High-resolution imaging of elastic properties using harmonic cantilevers. Sensors and Actuators a-Physical, 2004. 114(2-3): p. 183-190.

11. Sarioglu, A.F., S. Magonov, and O. Solgaard, Tapping-mode force spectroscopy using cantilevers with interferometric high-bandwidth force sensors. Applied Physics Letters, 2012. 100(5).

12. Sarioglu, A.F. and O. Solgaard, Modeling, design, and analysis of interferometric cantilevers for time-resolved force measurements in tapping-mode atomic force microscopy. Journal of Applied Physics, 2011. 109(6).

13. Garcia, R. and E.T. Herruzo, The emergence of multifrequency force microscopy. Nature Nanotechnology, 2012. 7(4): p. 217-226.

14. Legleiter, J., J.D. Fryer, D.M. Holtzman, and T. Kowalewski, The Modulating Effect of Mechanical Changes in Lipid Bilayers Caused by ApoE-Containing 
Lipoproteins on A $\beta$ Induced Membrane Disruption. ACS Chemical Neuroscience, 2011. 2: p. 588-599.

15. Milhiet, P.-E., F. Gubellini, A. Berquand, P. Dosset, J.-L. Rigaud, C. Le Grimellec, and D. Lévy, High-Resolution AFM of Membrane Proteins Directly Incorporated at High Density in Planar Lipid Bilayer. Biophysical Journal, 2006. 91(9): p. 3268-3275.

16. Shamitko-Klingensmith, N., K.M. Molchanoff, K.A. Burke, G.J. Magnone, and J. Legleiter, Mapping the Mechanical Properties of Cholesterol-Containing Supported Lipid Bilayers with Nanoscale Spatial Resolution. Langmuir, 2012. 28 : p. 13411-13422.

17. Leonenko, Z., E. Finot, and D. Cramb, AFM study of interaction forces in supported planar DPPC bilayers in the presence of general anesthetic halothane. Biochimica et Biophysica Acta - Biomembranes, 2006. 1758(4): p. 487-492.

18. Pifer, P.M., E.A. Yates, and J. Legleiter, Point mutations in A $\beta$ result in the formation of distinct polymorphic aggregates in the presence of lipid bilayers. PLoS One, 2011. 6: p. e16248.

19. Espinosa, G., I. Lopez-Montero, F. Monroy, and D. Langevin, Shear rheology of lipid monolayers and insights on membrane fluidity. Proceedings of the National Academy of Sciences of the United States of America, 2011. 108: p. 6008-6013, S6008/1-S6008/3.

20. vanMeer, G., D.R. Voelker, and G.W. Feigenson, Membrane lipids: where they are and how they behave. Nature Reviews Molecular Cell Biology, 2008. 9: p. 112-124.

21. Kranenburg, M. and B. Smit, Phase Behavior of Model Lipid Bilayers. The Journal of Physical Chemistry B, 2005. 109(14): p. 6553-6563.

22. Suresh, S. and J.M. Edwardson, Phase separation in lipid bilayers triggered by low pH. Biochemical and Biophysical Research Communications, 2010. 399(4): p. 571-574.

23. Mansilla, M.C., L.E. Cybulski, D. Albanesi, and D. de Mendoza, Control of Membrane Lipid Fluidity by Molecular Thermosensors. Journal of Bacteriology, 2004. 186(20): p. 6681-6688. 
24. Simons, K. and W.L.C. Vaz, Model systems, lipid rafts, and cell membranes. Annual Review of Biophysics and Biomolecular Structure, 2004. 33: p. 269-295.

25. Hutter, J.L. and J. Bechhoefer, Calibration of atomic-force microscope tips. Review of Scientific Instruments, 1993. 64(7): p. 1868-1873.

26. Kowalewski, T. and J. Legleiter, Imaging stability and average tip-sample force in tapping mode atomic force microscopy. J. Appl. Phys., 2006. 99: p. 064903/1064903/5.

27. Kumar, B., P.M. Pifer, A. Giovengo, and J. Legleiter, The effect of set point ratio and surface Young's modulus on maximum tapping forces in fluid tapping mode atomic force microscopy. J. Appl. Phys., 2010. 107.

28. Van Dijck, P.W.M., B. De Kruijff, L.L.M. Van Deenen, J. De Gier, and R.A. Demel, The preference of cholesterol for phosphatidylcholine in mixed phosphatidylcholine-phosphatidylethanolamine bilayers. Biochimica et Biophysica Acta - Biomembranes, 1976. 455(2): p. 576-587.

29. Epand, R.M. and R. Bottega, Modulation of the phase transition behavior of phosphatidylethanolamine by cholesterol and oxysterols. Biochemistry, 1987. 26(7): p. 1820-1825.

30. Kučerka, N., M.-P. Nieh, and J. Katsaras, Fluid phase lipid areas and bilayer thicknesses of commonly used phosphatidylcholines as a function of temperature. Biochimica et Biophysica Acta - Biomembranes, 2011. 1808(11): p. 2761-2771.

31. Pan, J., S. Tristram-Nagle, N. Ku erka, and J.F. Nagle, Temperature Dependence of Structure, Bending Rigidity, and Bilayer Interactions of Dioleoylphosphatidylcholine Bilayers. Biophysical Journal, 2008. 94(1): p. 117124.

32. Yip, C.M., E.A. Elton, A.A. Darabie, M.R. Morrison, and J. McLaurin, Cholesterol, a Modulator of Membrane-associated A $\beta$-Fibrillogenesis and Neurotoxicity. Journal of Molecular Biology, 2001. 311: p. 723-734.

33. Jass, J., T. Tjarnhage, and G. Puu, From liposomes to supported, planar bilayer structures on hydrophilic and hydrophobic surfaces: An atomic force microscopy study. Biophysical Journal, 2000. 79(6): p. 3153-3163. 
34. Bar, G., Y. Thomann, R. Brandsch, H.J. Cantow, and M.H. Whangbo, Factors affecting the height and phase images in tapping mode atomic force microscopy. Study of phase-separated polymer blends of poly(ethene-co-styrene) and poly(2,6dimethyl-1,4-phenylene oxide). Langmuir, 1997. 13(14): p. 3807-3812.

35. Garcia, R., R. Magerle, and R. Perez, Nanoscale compositional mapping with gentle forces. Nature Materials, 2007. 6(6): p. 405-411.

36. Magonov, S.N., V. Elings, and M.H. Whangbo, Phase imaging and stiffness in tapping-mode atomic force microscopy. Surface Science, 1997. 375(2-3): p. L385-L391.

37. Melcher, J., C. Carrasco, X. Xu, J.L. Carrascosa, J. Gomez-Herrero, P.J. de Pablo, and A. Raman, Origins of phase contrast in the atomic force microscope in liquids. Proceedings of the National Academy of Sciences of the United States of America, 2009. 106(33): p. 13655-13660.

38. Zitzler, L., S. Herminghaus, and F. Mugele, Capillary forces in tapping mode atomic force microscopy. Physical Review B, 2002. 66(15): p. 155436.

39. Garcia, R., J. Tamayo, and A. San Paulo, Phase contrast and surface energy hysteresis in tapping mode scanning force microsopy. Surface and Interface Analysis, 1999. 27(5-6): p. 312-316.

40. Stark, M., C. Möller, D.J. Müller, and R. Guckenberger, From Images to Interactions: High-Resolution Phase Imaging in Tapping-Mode Atomic Force Microscopy. Biophysical Journal, 2001. 80(6): p. 3009-3018.

41. Ebeling, D. and S.D. Solares, Amplitude modulation dynamic force microscopy imaging in liquids with atomic resolution: comparison of phase contrasts in single and dual mode operation. Nanotechnology, 2013. 24(13).

42. Scott, W.W. and B. Bhushan, Use of phase imaging in atomic force microscopy for measurement of viscoelastic contrast in polymer nanocomposites and molecularly thick lubricant films. Ultramicroscopy, 2003. 97(1-4): p. 151-169.

43. Melcher, J., C. Carrasco, X. Xu, J.L. Carrascosa, J. Gómez-Herrero, P.J.d. Pablo, A. Raman, and J.-P. Aime, Origins of Phase Contrast in the Atomic Force Microscope in Liquids. Proceedings of the National Academy of Sciences of the United States of America, 2009. 106(33): p. 13655-13660. 
44. Basak, S. and A. Raman, Dynamics of tapping mode atomic force microscopy in liquids: Theory and experiments. Applied Physics Letters, 2007. 91(6): p. 064107-3.

45. Mueller, D.J. and A. Engel, Atomic force microscopy and spectroscopy of native membrane proteins. Nature Protocols, 2007. 2(9): p. 2191-2197.

46. Voitchovsky, K., S.A. Contera, M. Kamihira, A. Watts, and J.F. Ryan, Differential stiffness and lipid mobility in the leaflets of purple membranes. Biophysical Journal, 2006. 90(6): p. 2075-2085.

47. van Noort, S.J.T., O.H. Willemsen, K.O. van der Werf, B.G. de Grooth, and J. Greve, Mapping electrostatic forces using higher harmonics tapping mode atomic force microscopy in liquid. Langmuir, 1999. 15(21): p. 7101-7107.

48. Burke, K.A., E.A. Yates, and J. Legleiter, Amyloid-Forming Proteins Alter the Local Mechanical Properties of Lipid Membranes. Biochemistry, 2013. 52(5): p. 808-817.

49. Guzman, H.V., A.P. Perrino, and R. Garcia, Peak forces in high-resolution imaging of soft matter in liquid. ACS Nano, 2013. 7(4): p. 3198-3204.

50. Yates, E.A., S.L. Owens, M.F. Lynch, E.M. Cucco, C.S. Umbaugh, and J. Legleiter, Specific domains of $A \beta$ facilitate aggregation on and association with lipid bilayers. Journal of Molecular Biology, 2013. 425(11): p. 1915-33.

51. Burke, K.A., K.J. Kauffman, C.S. Umbaugh, S.L. Frey, and J. Legleiter, The interaction of polyglutamine peptides with lipid membranes is regulated by flanking sequences associated with huntingtin. The Journal of Biological Chemistry, 2013. 288(21): p. 14993-5005.

52. Burke, K.A., K.M. Hensal, C.S. Umbaugh, M. Chaibva, and J. Legleiter, Huntingtin disrupts lipid bilayers in a polyQ-length dependent manner. Biochimica Et Biophysica Acta, 2013. 1828(8): p. 1953-61.

53. Chaibva, M., K.A. Burke, and J. Legleiter, Curvature Enhances Binding and Aggregation of Huntingtin at Lipid Membranes. Biochemistry, 2014. 53(14): p. 2355-2365. 
54. Payam, A.F., J.R. Ramos, and R. Garcia, Molecular and Nanoscale Compositional Contrast of Soft Matter in Liquid: Interplay between Elastic and Dissipative Interactions. ACS Nano, 2012. 6(6): p. 4663-4670. 


\section{Microtubule modification influences the cellular response to amyloid- $\beta_{1-42}$ aggregates}

During the normal aging process, cytoskeletal changes such as a reduction in density or disruption of cytoskeletal components occur that can affect proper neuronal functioning. Considering that aging is the biggest risk factor for Alzheimer's disease (AD), this study sought to determine how microtubule (MT) modifications influence the cellular response to amyloid- $\beta_{1-42}\left(A \beta_{1-42}\right)$, a toxic peptide implicated in development of AD. First it was necessary to obtain populations of hypothalamic GT1-7 neurons with a variety of MT states. Specifically, neuronal MTs were modified by common disrupting or stabilizing drugs, and then the physical and mechanical properties of the modified neurons were determined. The MT modified neurons were then exposed to $A \beta_{1-42}$ and the ability of the neurons to cope with the exposure to the toxic aggregates was determined by a variety of biochemical assays. Flow cytometry studies indicated that MT disruption reduced the ability of the aggregates to bind to the plasma membrane by $45 \%$ per cell compared to neurons with stabilized or unaltered MTs; however, populations of neurons with disrupted MTs experienced over 3 times the amount of cytotoxicity induced by $A \beta_{1-42}$ aggregates. On the contrary, MT stabilization delayed toxicity caused by $A \beta_{1-42}$ aggregates. These results demonstrate that MT modifications significantly influence the ability of neurons to cope with toxicity induced by $A \beta_{1-42}$ aggregates.

\subsection{Introduction}

The cytoskeleton plays a critical role in the complex morphology of neurons, intra- and intercellular signaling, and organelle transport [1]. In neurons, there are three major cytoskeletal components, neurofilaments, actin, and microtubules [2]. Neurofilaments (NFs) are found within the axon and dendrites, where they provide structural support, aid in axonal transport, and regulate axon diameter [3,4]. The inner plasma membrane of neurons is lined with short fragments of filamentous actin (F-actin), while the dendrites and synaptic terminals are rich in F-actin bundles and lattices [1]. Lastly, microtubules (MTs) span the length of the axon and protrude into the base of dendrites, where they provide structural support, and function as tracks for organelle transport [5]. Importantly, each of these cytoskeletal components experience dysfunction with aging that can alter 
cellular functioning [2, 6-12], but this chapter will focus on the microtubule component of the cytoskeleton.

There are many examples of age-related microtubule modifications throughout the body, and a select few will be described here for illustrative purposes. In the organ of Corti, the isoform levels of tubulin changed with age, and there was a decrease in the number and length of microtubules [13]. These changes alter cellular mechanical properties, leading to a reduction in the conduction of sound-induced vibrations that are associated with hearing [13]. In fibroblasts, reorganization of the MT network occurred with aging, which has implications for cell division [6, 14, 15]. Additionally, a reduction in density of the microtubule lattice was observed in aged mouse muscle fibers [16]. This reduction could alter nuclear shape and distribution in muscle cells, leading to decreased cellular functioning or even apoptosis [16]. These findings demonstrate that age-induced MT modifications vary depending on the type of cell.

In neurons, exposure to the lipid peroxidation product 4-hydroxy-2(E)-nonenal (HNE, a common product of age-related oxidative stress) caused extensive MT disruption, and HNE adduction with tubulin prevented polymerization [17]. In human studies, tissue biopsied from cognitively healthy adults showed an age-dependent decrease in the microtubule density of pyramidal neurons [18]. Together, these findings demonstrate that neuronal MTs are critically affected during aging. This may have implications for the development of Alzheimer's disease (AD), as AD brains display a significant reduction in pyramidal neuron density [19]. MT modification is associated with mitochondrial abnormalities [20,21], and reduced axonal transport resulting from MT disruption could also contribute to the loss of synaptic connectivity between neurons that causes cognitive impairments in $\mathrm{AD}[18,22]$. Considering that aging is the major risk factor for $\mathrm{AD}$ [23], it would be beneficial to gain a more complete understanding of how age-related MT modifications in neurons contribute to the disease.

Two pathological hallmarks of $\mathrm{AD}$ are extracellular plaques of amyloid- $\beta$ (A $\beta$ ) and intraneuronal neurofibrillary tangles (NFTs) containing the hyperphosphorylated microtubule-associated protein Tau (Tau) [24]. A $\beta$ is a 38-43 amino acid long residue that is produced from sequential cleavages of the amyloid precursor protein (APP) [25], but $A \beta_{1-40}$ and $A \beta_{1-42}$ are the most abundant forms of the peptide. Several findings 
support the notion that $A \beta_{1-42}$ is the more pathological form of the peptide: $A \beta_{1-42}$ forms aggregates more rapidly than $A \beta_{1-40}[26,27], A \beta_{1-42}$ is the predominate species found in plaques $[28,29], A \beta_{1-40}$ can slow or inhibit $A \beta_{1-42}$ from aggregating [30-32], an increased ratio of $\mathrm{A} \beta_{1-42}: \mathrm{A} \beta_{1-40}$ produced toxic effects in vitro and in vivo [33-35], and $\mathrm{A} \beta_{1-42}$ itself was associated with greater neurotoxic effects compared to $A \beta_{1-40}$ in mice [36]. Importantly, these findings demonstrate that $A \beta$ aggregates themselves are toxic, but their presence is also thought to trigger a cascade of events leading to the formation of NFTs $[25,37,38]$, which ultimately causes the deterioration of the neuronal processes and cell death [39-44].

In the brains of $\mathrm{AD}$ individuals, age-related neuronal modifications are amplified in selectively vulnerable regions of the brain, such as the hippocampus and closely related limbic and cortical structures [23, 45, 46]. These changes can contribute to the increased production or decreased clearance of $A \beta$, resulting in $A \beta$-induced neurotoxicity [23]. Specifically, impaired axonal transport, a consequence of age-related MT disruption, has been shown to stimulate the production and accumulation of $A \beta$ [47]. Therefore, although it is well known that $\mathrm{A} \beta$ has downstream effects on Tau and subsequently MT stability, age-related MT disruption may actually be a causative factor in $\mathrm{AD}$. Due to the relationship between aging, $\mathrm{A} \beta$, and $\mathrm{AD}$, we sought to determine how microtubule modifications influence the cellular response to toxicity caused by aggregates of $A \beta_{1-42}$. To accomplish this goal, GT1-7 neurons were treated with a variety of MT stabilizing or destabilizing agents, and the toxic ability of $\mathrm{A} \beta_{1-42}$ aggregates was examined. This work provides more complete understanding of how MT modification, which is a common feature of the natural aging process, may contribute to the development of AD.

\subsection{Methodology}

\subsubsection{Neuronal culture}

GT1-7 hypothalamic neurons were grown in DMEM medium supplemented with $4.5 \mathrm{~g} / \mathrm{L}$

glucose, $110 \mathrm{mg} / \mathrm{L}$ sodium pyruvate, $1 \%$ penicillin-streptomycin (stabilized with 10,000 units penicillin and $10 \mathrm{mg}$ streptomycin/mL), $2 \mathrm{~g} / \mathrm{L}$ sodium bicarbonate, $584 \mathrm{mg} / \mathrm{L} \mathrm{L}$ glutamine (Sigma-Aldrich, St. Louis, MO), $5 \mathrm{mM}$ HEPES (Fisher BioReagents, 
Waltham, MA) and 10\% HyClone ${ }^{\circledR}$ fetal bovine serum (Thermo Scientific, Waltham, MA).[48-52] Cultures were maintained at $37{ }^{\circ} \mathrm{C}$ and $5 \% \mathrm{CO}_{2}$. For all cell-based experiments, serum concentration was reduced to $5 \%$.

\subsubsection{Microtubule treatments and $A \beta_{1-42}$ preparation}

Nocodazole, colchicine, and paclitaxel (Fisher Scientific) were dissolved in dimethyl sulfoxide (DMSO, Fisher Scientific) and further diluted in the appropriate buffer or medium to a final concentration of $0.1-10 \mu \mathrm{M}$. Neurons were exposed to a microtubule (MT) treatment for 4 hours prior to $A \beta$ exposure to ensure cytoskeletal modification. $A \beta$ peptides $\left(\mathrm{A} \beta_{1-42}\right.$ and FAM-labeled $\left.\mathrm{A} \beta_{1-42}\right)$ were purchased from AnaSpec Inc., Fremont, CA. Lyophilized peptides were stored at $-20{ }^{\circ} \mathrm{C}$ and allowed to equilibrate to room temperature for 30 minutes before resuspension in 1,1,1,3,3,3-hexafluoro-2-propanol (HFIP, Fisher Scientific) to $1 \mathrm{mM}$. The peptide solution was sonicated for 10 minutes at room temperature and aliquoted into microcentrifuge tubes. The solution was dried and the peptide films were stored at $-20{ }^{\circ} \mathrm{C}$ until use. Directly before use, HFIP-treated peptide films were dissolved in DMSO by pipette mixing, vortexing, and sonication for 10 minutes at $37{ }^{\circ} \mathrm{C}$.[53] Peptide solutions were then diluted into the appropriate buffer or medium to a final concentration of $5 \mu \mathrm{M}$. DMSO exposure never exceeded $1 \%$.

\subsubsection{Fluorescence microscopy}

\section{Tubulin visualization}

After attachment, the medium was replaced with fresh medium containing CellLight ${ }^{\circledR}$ Tubulin-RFP BacMam 2.0 (Molecular Probes) at a concentration of 30 particles per cell. Neurons were incubated with the RFP for 48 hours, then were then exposed to treatments as earlier described. Cells were rinsed remove any unbound peptide, then cells were fixed with $0.3 \%$ glutaraldehyde in a BRB80 buffer. BRB80 buffer was prepared with $80 \mathrm{mM}$ PIPES (1,4-piperazinediethanesulfonic acid) at $\mathrm{pH}$ 6.9, $1 \mathrm{mM}$ EGTA (ethyleneglycol bis(aminoethylether) tetraacetic acid) and $1 \mathrm{mM}$ magnesium chloride (Fisher BioReagents). Coverslips were treated with sodium borohydride to reduced unwanted autofluorescence, and slides were prepared as described above. 


\section{A $\beta_{1-42}$ membrane binding}

Neurons were seeded on $22 \mathrm{~mm}$ poly-D-lysine coated glass coverslips and allowed to adhere overnight. After attachment, neurons were treated with a MT-altering drug or vehicle for 4 hours and then exposed to FAM-A $\beta$ for 30 minutes. Cells were rinsed and incubated with CellLight ${ }^{\circledR}$ Deep Red Plasma Membrane stain for 10 minutes at $37^{\circ} \mathrm{C}$. Fixation was performed with paraformaldehyde under nonpermeabilizing conditions and coverslips were mounted onto glass slides with ProLong® Gold antifade reagent containing DAPI (Molecular Probes). Slides were allowed to cure for 24 hours in the dark before imaging (Leica Microsystems, Buffalo Grove, IL).

\subsubsection{Cellular mechanical evaluation via AFM}

Cells were seeded on poly-D-lysine coated glass coverslips and allowed to incubate overnight. Neurons were treated with a MT-altering drug or vehicle for 4 hours and then exposed to $A \beta$ for 30 minutes. Cells were washed and fixed in a glutaraldehyde solution [54]. Coverslips were mounted onto the AFM stage and imaged in buffer with a Nanoscope V MultiMode scanning probe microscope (Veeco, Santa Barbara, CA) equipped with a closed-loop vertical engage J-scanner. Experiments were conducted in contact and force volume imaging modes. All images were acquired using a VISTAprobe contact mode short cantilever (Nanoscience Instruments, Inc., Phoenix, AZ), where the tip radius was $10 \mathrm{~nm}$ and the spring constant was calculated via the thermal tuning method prior to each experiment $[48,55]$. Scan speed for topography images was $0.5 \mathrm{~Hz}$ with a pixel resolution of $512 \times 512$. Force volume images were acquired at $10 \mathrm{~Hz}$, with a resolution of $128 \times 128$ curves and given these parameters, it took approximately 1 hour to obtain a force map. Young's modulus (E) was evaluated using NanoScope Analysis software v1.5 (Bruker, Santa Barbara, CA). A Hertz model was applied to determine E, where Poisson's ratio was $v=0.5[49,54]$. A minimum of 9 cells were evaluated for each sample. E values were extracted from both the nuclear region and the cellular projections. Histograms were produced in MATLAB (Math Works Inc., Natick, MA) and values are given as mean \pm standard deviation. Statistical significance was analyzed using an unpaired t-test, where a difference at $p<0.05$ was considered statistically significant. 


\subsubsection{Assessment of $A \beta_{1-42}$ aggregation state}

To determine the $A \beta$ aggregate morphology, $A \beta$ was prepared as described above and diluted in PBS to $5 \mu \mathrm{M}$. The solution was spotted onto freshly cleaved mica after 0,24 , and 48 hours of incubation at $37{ }^{\circ} \mathrm{C}$. Mica was rinsed with ultrapure water and immediately dried under a gentle stream of nitrogen. Ex situ images were acquired via tapping mode AFM with a VISTAprobe silicon cantilever with a nominal spring constant of $40 \mathrm{~N} / \mathrm{m}$ and a resonance frequency of $\sim 300 \mathrm{kHz}$. AFM image analysis was performed using the image processing toolbox of Matlab, as previously described [56]. The images were imported into Matlab and flattened to correct for background curvature. Using a height threshold, a binary map of the surface was created to locate individual aggregates and by implementing pattern recognition algorithms to the binary map, aggregate features were measured (height, volume, etc.).

The absence of fibrillar aggregates was confirmed using a SensoLyte ${ }^{\circledR}$ Thioflavin $\mathrm{T}$ (ThT) A $\beta$ aggregation kit (Anaspec, Fremont, CA). $5 \mu \mathrm{M}$ A $\beta$ was prepared as earlier described and added to an untreated 96 well plate containing a ThT solution. Pretreated $\mathrm{A} \beta$ from the supplier was prepared as recommended and used as a control. The fluorescence intensity was monitored via an Infinite M1000 Pro microplate reader (Tecan US, Raleigh, NC) at Ex/Em of $440 / 484 \mathrm{~nm}$ every 10 minutes over 48 hours at $37{ }^{\circ} \mathrm{C}$ with no shaking to mimic the conditions of the plasma membrane degradation and MTT reduction assays.

\subsubsection{Flow cytometry}

Neurons were seeded in 6-well plates and allowed to incubate overnight. A 4 hour MT or vehicle treatment was followed by an 80 minute exposure to FAM-A $\beta$.[57, 58] Samples were thoroughly washed to remove any unbound peptide and they were lifted from the surface using a $0.25 \%$ trypsin solution. Neurons were then rinsed and resuspended in cold sorting buffer (1x D-PBS, $25 \mathrm{mM}$ HEPES at $\mathrm{pH}$ 7.0, $2.5 \mathrm{mM}$ EDTA, and 1\% bovine serum albumin). The fluorescence intensity per cell was assessed with a BD FACSCalibur cytometer and BD FACSDiva v8.0 software (BD Biosciences, Franklin Lakes, NJ). 


\subsubsection{Plasma membrane degradation assay}

Neurons were seeded at 10,000 cells/100 $\mu \mathrm{L}$ medium on a tissue culture-treated 96-well plate and allowed to incubate for 24 hours. Cells received a MT modifying pretreatment, followed by an $A \beta$ exposure for an additional 24 or 48 hours. Ethidium homodimer-1 (EthD-1, Molecular Probes, Grand Island, NY) was used to evaluate plasma membrane degradation according to the manufacturer's protocol. EthD-1 enters neurons with compromised membranes to produce a bright red fluorescence upon binding to nucleic acids. As a control, a group of vehicle-treated cells received a treatment with $70 \%$ methanol 30 minutes prior to EthD-1 exposure. A $6 \mu \mathrm{M}$ stock of EthD-1 was prepared in Dulbecco's phosphate buffered saline (D-PBS, Fisher Scientific) and $100 \mu \mathrm{L}$ was added to each well for a final concentration of $3 \mu \mathrm{M}$. The samples were incubated for 45 minutes at room temperature and the fluorescence intensity was measured at Ex/Em of $495 / 635 \mathrm{~nm}$. All assay measurements were performed at least in triplicate.

\subsubsection{MTT reduction assay}

Samples were prepared exactly as described for the cytotoxicity assay and MTT reduction was evaluated via the MTT assay kit (Abnova, Walnut, CA). The MTT assay involves the conversion of MTT (3-(4,5-dimethylthiazol-2-yl)-2,5-diphenyltetrazolium bromide) to insoluble formazan crystals. The tetrazolium salt MTT reduction is dependent on reducing agents, NADH and NADPH, produced by metabolically active cells. MTT reagent was added to each well according to the manufacturer's protocol and the samples were incubated for 4 hours at $37^{\circ} \mathrm{C}$. The crystals were solubilized by adding $100 \mu \mathrm{L}$ of the provided buffer solution to each well with gentle shaking for 1 hour at room temperature. The absorbance was measured at $570 \mathrm{~nm}$.

\subsubsection{Statistical analysis}

Plasma membrane degradation and MTT reduction was analyzed using GraphPad Prism v5 software (GraphPad Software Inc., La Jolla, CA). Statistical significance was determined using a one-way analysis of variance (ANOVA) with Turkey's multiple comparison test. Differences at $p<0.05$ were considered statistically significant. All 
assay data is plotted as a ratio of value/baseline (vehicle control) where error bars reflect standard error of the mean.

\subsection{Results}

\subsubsection{Microtubule modification alters the physical and mechanical properties of neurons}

To determine how the MT network influences the ability of $A \beta_{1-42}$ aggregates to cause toxicity, it was first necessary to induce MT modification by common disrupting or stabilizing treatments, and then verify that cellular changes had occurred to validate the model system. Hypothalamic GT1-7 neurons were treated with a MT disruptor (colchicine or nocodazole), or stabilizer (paclitaxel) for four hours at $0.1,1.0$, and 10.0 $\mu \mathrm{M}$. Both colchicine and nocodazole cause MT depolymerization at high concentrations [59], while paclitaxel mechanically stabilizes MTs by binding to $\beta$-tubulin [60]. After four hours of treatment, the physical and mechanical properties of the neurons were examined. Neurons with chemically disrupted MTs displayed morphological changes, specifically in the neurites (Fig. 1a-b). This observation is in agreement with other studies that reported MT disruption induced morphological alterations in the periphery of PC-12 and endothelial cells [61-63].

To determine the mechanical impact of MT modification, the Young's modulus (E, a measure of stiffness) of individual neurons was mapped by AFM-based force volume imaging (Fig. 2a). After four hours of treatment with an MT-modifier, neurons were fixed with glutaraldehyde to effectively preserve cell surface features [64]. Fixation was necessary due to instrumental limitations, i.e., the length of time required to produce a force map, but it is common practice for AFM-based cellular experiments, as it improves image resolution and consistency in mechanical measurements [54, 65-72]. Fixation is known to increase cellular stiffness [54, 69]; therefore, these studies reflect the relative changes in $\mathrm{E}$. That is, the values of $\mathrm{E}$ reported in this study are not biologically accurate, but fixation allows for fair comparisons of stiffness across samples whereas the properties of living cells may change during the mapping. Relative values of E were determined by extracting individual force- distance curves from the mechanical surface maps. Curves were selected from two neuronal regions, the soma and the 


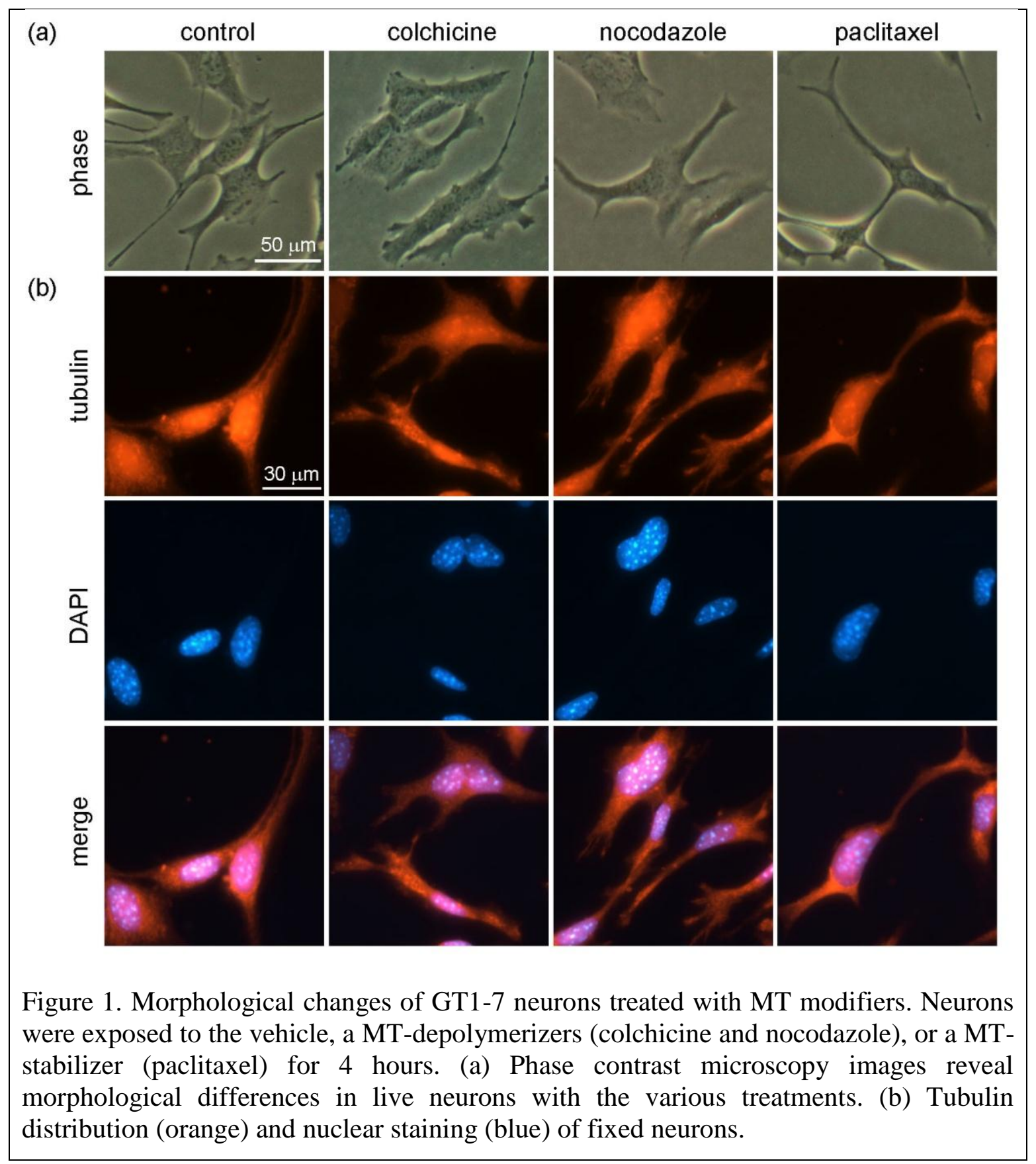

processes, to produce E histograms for each sample (Fig. 2b-c). Histograms represent E data extracted from a minimum of 9 neurons for each treatment. Treatment with $10 \mu \mathrm{M}$ colchicine for four hours significantly decreased $\mathrm{E}$ in the somatic region by $31 \%$ (Fig. $2 b)$. Literature reports on the mechanical effect of colchicine are conflicting. For instance, neutrophils treated with similar concentrations of colchicine experienced 
significant stiffening [73]. In NRK fibroblasts, however, no mechanical alteration or change in stress fibers was observed after treatment with $100 \mu \mathrm{M}$ colchicine [74]. Furthermore, vascular smooth muscle cells exhibited a significant decrease in cell stiffness when treated with colchicine [75]. Thus, the influence of colchicine on cellular mechanics is highly dependent on cell type, the state of cell division, and even on the region of the cell. Unlike the somatic region, after colchicine treatment the rigidity of the neuronal processes was not significantly different than that of the vehicle-treated control (1.8\% difference). The effect of softening in the somatic region while retaining stiffness

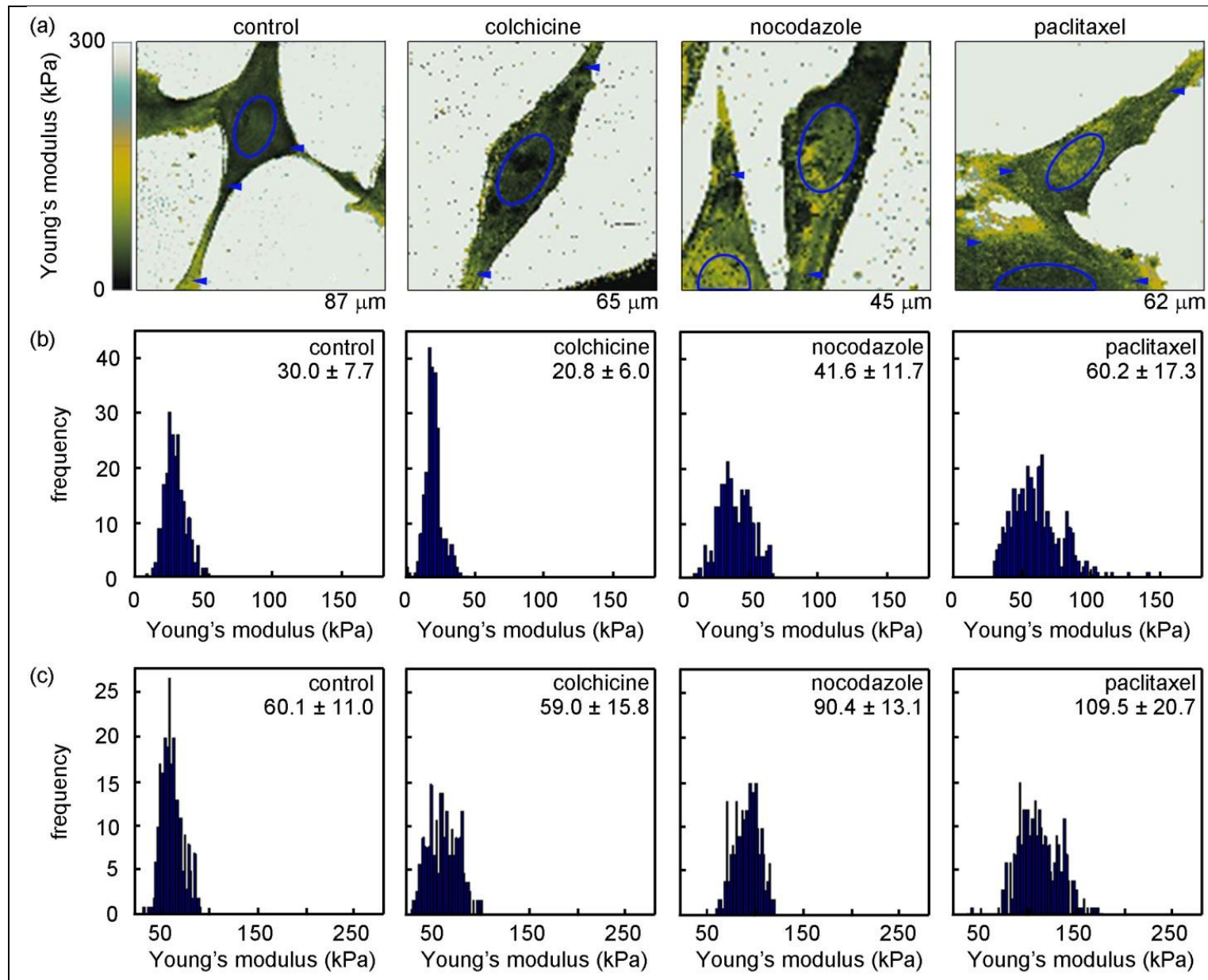

Figure 2. Measuring the Young's modulus of neurons treated with MT modifiers. (a) Examples of Young's modulus (E) maps obtained by force volume imaging. Histograms of the average value of $E$ were constructed by extracting values from the (b) soma or (c) processes of neurons treated with: the vehicle or MT-altering drug (colchicine, nocodazole, paclitaxel) for 4 hours. Measurements represent the mean $\mathrm{E} \pm$ standard deviation. 
in the neurites can be related to previous studies on fibroblasts. When aged hamster fibroblasts were treated with a microtubule disruptor, centrifugal depolymerization occurred and MT remnants were found in the cortical areas [14]. The reasoning for this effect is unknown and could be attributed to several factors (aging may disrupt the centrosome, reverse the polarity of MTs, alter the stability of the anchorage to the membrane, etc.) [14]. Given this information, it seems as though colchicine prevents polymerization in the processes, while dissociation occurs at the minus end of MTs, near the centrosome.

Unlike colchicine, when cells were treated with $10 \mu \mathrm{M}$ nocodazole, the stiffness of the soma and processes increased by $39 \%$ and $50 \%$, respectively, as compared to the vehicle-treated control (Fig. 2b-c). Our findings are in agreement with findings from similar studies on L929 fibroblast-like where an increase in cellular stiffness was observed after treatment with nocodazole [76]. This was attributed to the reorganization of the cytoskeletal network after MT depolymerization. Cytoskeletal reorganization after injury was observed in Swiss 3T3 cells; when cells were treated with MT disruptors, actin polymerization occurred in a dose- and time-dependent manner [77]. These findings demonstrate that cell stiffening after nocodazole-induced MT disruption is a consequence of actin polymerization. The discrepancy in mechanical changes between colchicine and nocodazole treated neurons may arise from the binding mechanisms to tubulin. Although nocodazole binds tubulin more rapidly than colchicine, colchicine binding is irreversible [78], which may explain the variation in cellular stiffness between the two treatments.

When neurons were treated with $10 \mu \mathrm{M}$ paclitaxel, rigidity increased by $101 \%$ and $89 \%$ in the somatic region and the processes, respectively, compared to the vehicletreated control sample (Fig. 2b-c). Our results are consistent with a similar study, where stiffening occurred in cortical neurons were exposed to $10 \mu \mathrm{M}$ paclitaxel [79]. Although other studies have demonstrated that MT-disruption may produce an increase in cellular stiffness by activating a secondary response of actin polymerization, paclitaxel-mediated MT stabilization was not reported to stimulate actin production [77, 80]. Therefore, the increase in cellular stiffness is likely due to the mechanical stabilization of the MT network. Taken together, these studies confirmed that a four hour exposure to MT modifiers altered the morphology and the mechanical stiffness of neurons. 


\subsubsection{Microtubule disruption reduces the ability of $A \beta_{1-42}$ to bind to the cell membrane}

Before investigating how MT modification influences $\mathrm{A} \beta_{1-42}$-induced cytotoxicity, it was first necessary to characterize the type of peptide aggregates used in the study. Knowledge of the peptide aggregation state is critical because the extent of membrane interaction [81, 82] and the resulting toxicity [83] is dependent on the aggregation state. A fresh $A \beta_{1-42}$ solution was prepared from lyophilized peptides using the Stine

(a)
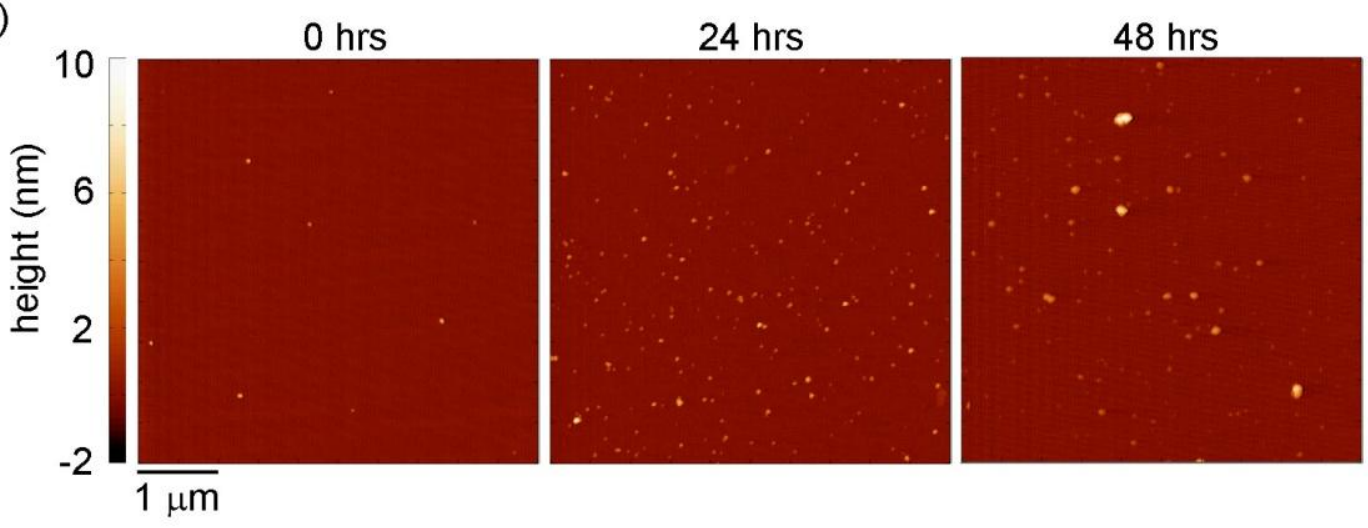

(b)

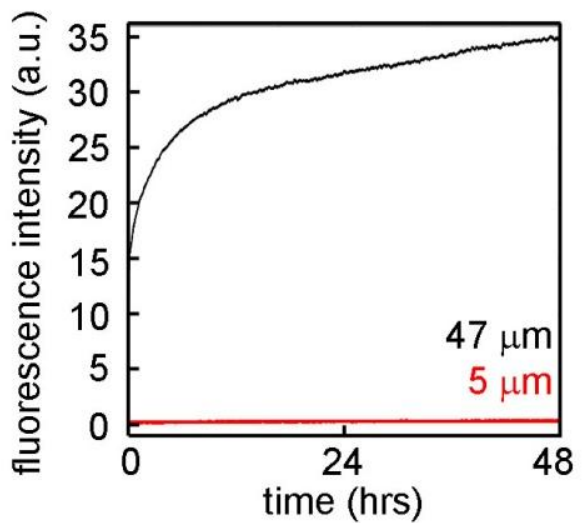

(c)

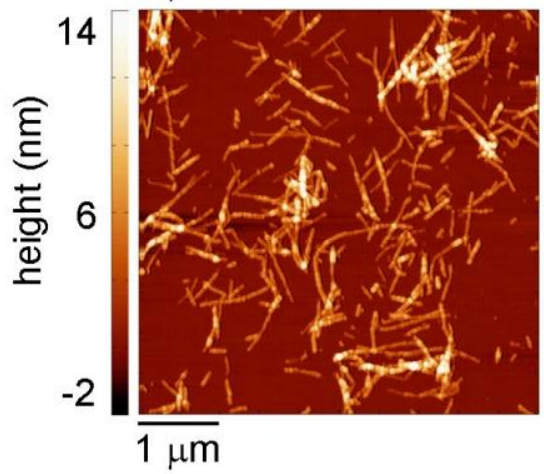

Figure 3. Assessment of the $A \beta_{1-42}$ aggregation state. (a) Oligomeric aggregates were observed when $5 \mu \mathrm{M} \mathrm{A} \beta_{1-42}$ was spotted onto freshly cleaved mica and analyzed via $e x$ situ AFM imaging after 0,24 or 48 hours of incubation at $37{ }^{\circ} \mathrm{C}$ in a physiologically relevant buffer. (b) The thioflavin $t$ assay was performed to detect the presence of $\beta$ sheet, a characteristic of fibrils. The red line corresponds to $5 \mu \mathrm{M} \mathrm{A} \beta$ which was prepared as described in the methods section, and the black line represents $47 \mu \mathrm{M} A \beta_{1-42}$ obtained from the SensoLyte ${ }^{\circledR}$ Aggregation kit and prepared as recommended by the manufacturer. (c) Fibrillar aggregates were observed when $47 \mu \mathrm{M} \mathrm{A} \beta_{1-42}$ was imaged via ex situ AFM after 48 hours of incubation. 
preparation method [53]. The solution was diluted to $5 \mu \mathrm{M}$, and the aggregation state of the peptide was determined by ex situ AFM imaging (Fig. 3a). Aggregates grew in average height over the 48 hour period from $3.0 \pm 1.3 \mathrm{~nm}$ to $4.2 \pm 1.2 \mathrm{~nm}$. The size of $\mathrm{A} \beta_{1-42}$ aggregates can vary considerably depending on the preparation method, but this height is consistent with other measurements of large $A \beta_{1-42}$ oligomers [84-86]. The average width of the aggregates increased from $32.3 \pm 1.1 \mathrm{~nm}$ to $40.4 \pm 1.3 \mathrm{~nm}$ over 48 hours, which is likely greater than the actual diameter of the aggregates due to the size of the cantilever tip. These measurements are within a comparable range of other AFMbased findings, considering the experimental variability [84-86]. Elongated fibrillar aggregates, which can be over $1 \mu \mathrm{m}$ in length [87], were not observed in any $5 \mu \mathrm{M}$ sample image. The absence of fibrils in the $5 \mu \mathrm{M}$ solution was confirmed by the Thioflavin T (ThT) fluorescence assay, which is based on the interaction between ThT and $\beta$-sheet secondary structure, a characteristic of amyloid fibrils (Fig. 3b). As a control, the ThT assay included a solution of $47 \mu \mathrm{M} \mathrm{A} \beta_{1-42}$ that was guaranteed to aggregate by the manufacturer, and this solution caused an immediate spike in fluorescence intensity. The increased fluorescence intensity of the $47 \mu \mathrm{M}$ solution indicated the presence of fibrils, which was confirmed by AFM after 48 hours of incubation (Fig. 3c).

It has been demonstrated that cells may exhibit resistance to $A \beta$-membrane binding based on specific cellular characteristics, such as the presence of apoptotic signaling molecules, cell size, stage of the cell cycle, and cytosolic ATP levels [57]. Specifically, when PC-12 cells or GT1-7 neurons were exposed to FITC-A $\beta_{1-42}$, the cells could be divided into three distinct subpopulations based on their ability to attract the peptide (those with no binding affinity, high binding affinity, or extra-high binding affinity) [57]. Taking this into consideration for our studies, it was then necessary to determine how the altered neuronal morphology and mechanical stiffness from MT modification influenced the ability of $A \beta_{1-42}$ peptides to bind to the cell membrane. Here, neurons were pretreated with an MT-modifier or a vehicle control (DMSO) for four hours, and then were exposed to the $5 \mu \mathrm{M}$ FAM-A $\beta_{1-42}$ solution for 80 minutes. Importantly, pretreatments were not removed from the culture medium during the additional 80 minute incubation to prevent MT recovery. After the 80 minute FAM-A $\beta_{1 \text { - }}$ 42 exposure, aggregate-membrane binding was evaluated by flow cytometry (Fig. 4a). 
Approximately $0.2 \%$ of the vehicletreated control had positive staining, which may be attributed to the natural fluorescence of the living neurons, likely due to flavoproteins that have a similar emission wavelength as FAM-A $\beta_{1-42}(\sim 521$ $\mathrm{nm})[88,89]$. Excluding the vehicletreated control which received no FAM-A $\beta_{1-42}$ exposure, the vast majority of the

neurons in each sample population had membrane bound FAM-A $\beta_{1-42}$ (Fig. 4a); however, aggregatemembrane binding for the entire sample population was slightly lower for neurons pretreated with colchicine $(95.2 \pm 0.5 \%, p<0.01$ to 0.001$)$ and nocodazole (96.5 \pm $0.1 \%, p<0.001)$ than those treated with the FAM-A $\beta_{1-42}$ only (98.4 \pm $0.1 \%)$. The population of neurons pretreated with paclitaxel experienced a similar level of aggregate-membrane binding (98.5 $\pm 0.1 \%, p>0.05)$ as the FAM-A $\beta_{1-}$ 42 control. This contrasts results from the previously mentioned study, and the discrepancy between the aggregate-membrane binding is likely due to the peptide preparation

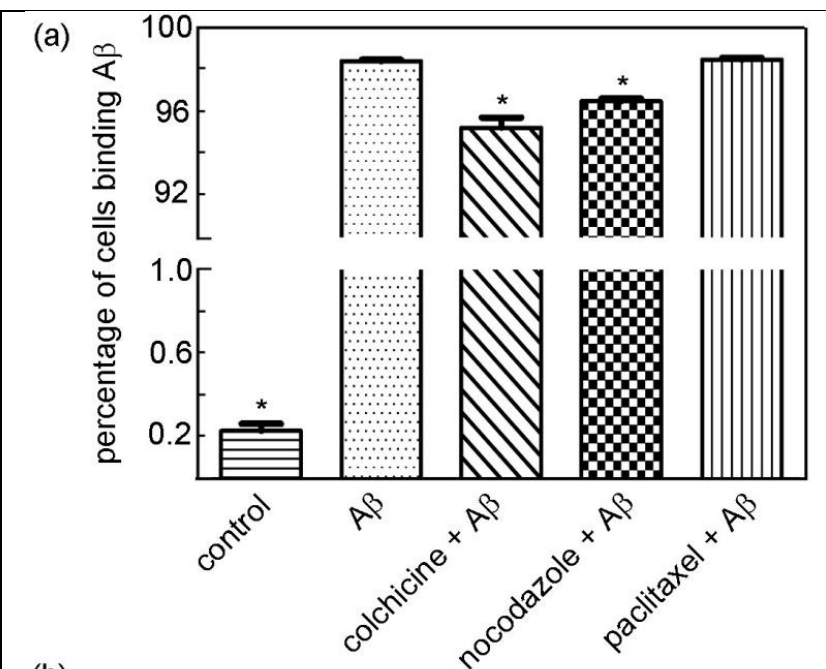

(b)

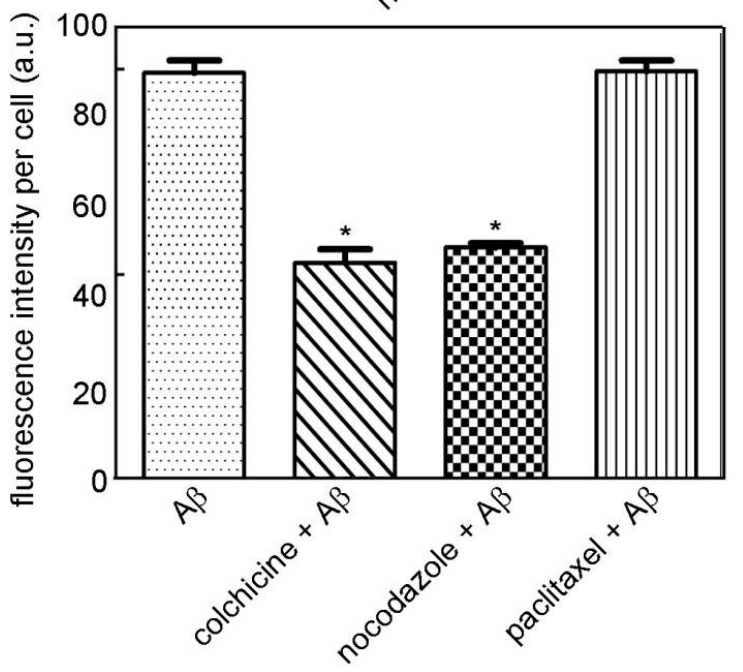

Figure 4. The extent of aggregate-membrane binding determined by flow cytometry. (a) The percentage of cells in each population that had membrane bound $A \beta_{1-42}$. (b) The amount of aggregate-membrane binding per cell. Neurons were treated with a vehicle or various MTaltering drugs (colchicine, nocodazole, paclitaxel) for 4 hours followed by an 80 minute incubation with FAM-A $\beta$ (with the exception of the vehicle control). Cells were extensively rinsed in physiological buffer to remove any unbound $A \beta$ before analysis. Measurements were made in triplicate, with 20,000 events per sample. Marker $(*)$ represents intensities found to be statistically different $(p<0.05)$ from the $\mathrm{A} \beta$ control and error bars represent standard error of the mean (SEM). 
technique, as it has been demonstrated that different aggregate species elicit distinctive membrane interactions.

Although over $95 \%$ of all the cells in each sample population (excluding the vehicle-treated control) had membrane bound FAM-A $\beta_{1-42}$, the extent of binding per cell varied based on the treatment (Fig. 4b). Similar to the results of the previous study examining whole population binding, the extent of aggregate-membrane binding per cell for neurons pretreated with paclitaxel was not statistically different than those treated with FAM-A $\beta_{1-42}$ only. However, neurons pretreated with colchicine and nocodazole bound approximately $45 \%$ less peptide per cell. This decrease in aggregate-membrane binding likely occurred due to the compromised state of neurons with MT disruption.

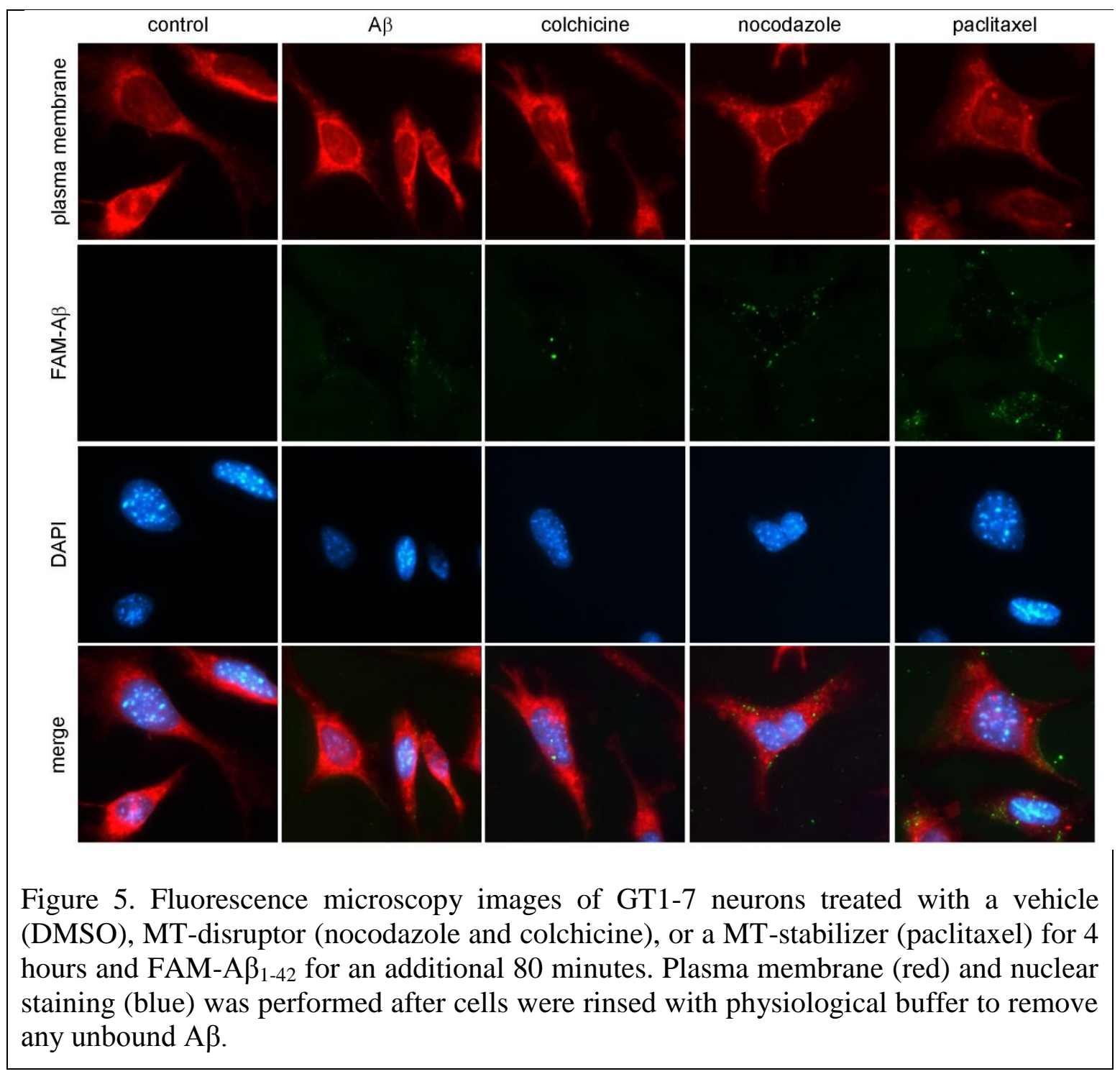


Although the neurites of the MT disrupted cells displayed morphological changes (Fig. 1), the reduction in binding was not a consequence of changes in the available membrane-binding area after MT modification. The area which the neurons occupied on the coverslip was examined by fluorescence microscopy, and there was no statistical difference between the treatment groups for the average area of each sample population.

To further examine the binding ability of the $A \beta_{1-42}$ aggregates after MT modification, fluorescence microscopy revealed that neurons pretreated with MTdisruptors bound FAM-A $\beta_{1-42}$ aggregates predominantly in the somatic region of the cell (Fig. 5). It is important to note that FAM-A $\beta_{1-42}$ had a punctate appearance in some of the fluorescence microscopy images (Fig. 5), and the punctate tightly adhered to the glass surface, as they could not be removed by extensive washing. The punctate appeared to be much larger than the aggregates observed by ex situ AFM studies. A plausible explanation for the size discrepancy is that the FAM-A $\beta_{1-42}$ aggregated more rapidly in the cell culture conditions than in the physiologically relevant buffers used for the ex situ AFM and ThT studies. Alternatively, the poly-D-lysine coated coverslips that the cells were cultured on for the fluorescence microscopy studies may have enhanced aggregation, as surfaces have been shown to influence the aggregation kinetics of $A \beta[86$, 90-92].

\subsubsection{Exposure to $A \beta_{1-42}$ alters neuronal rigidity}

Numerous studies have demonstrated that $A \beta$ causes membrane disruption, which can lead to structural and functional cellular changes [93-99]. With this in mind, surface maps of $\mathrm{E}$ were produced by force volume imaging to determine how $\mathrm{A} \beta_{1-42}$ aggregatemembrane binding contributes to the mechanical disruption of neurons. Neurons were pretreated with a MT modifier or the vehicle (DMSO) for four hours, and then exposed to $A \beta_{1-42}$ aggregates for an

\begin{tabular}{|l|cc|}
\hline Sample/Region & Soma (\% change) & Processes (\% change) \\
\hline Control & 142 & 154 \\
Colchicine & 143 & 29 \\
Nocodazole & 69 & 19 \\
Paclitaxel & 88 & 77 \\
\hline
\end{tabular}

Table 1. Percentage difference in Young's modulus (E) after exposure to $A \beta_{1-42}$ aggregates for 30 minutes. Changes were calculated for both the soma and neuronal processes. E increased significantly after treatment, with $p<0.001$. 
additional 30 minutes. Similar to the previously described flow cytometry and fluorescence microscopy experiments, during the incubation period with the peptide solution, the MT modifiers were not removed from the culture medium to prevent MT recovery. For all sample populations, the E of the soma (Fig. 6b) and processes (Fig. 6c) significantly increased after $A \beta_{1-42}$ aggregate exposure, where the percentage change in $\mathrm{E}$ after exposure to $A \beta_{1-42}$ aggregates in both neuronal regions is given in Table 1 , and directly comparable $\mathrm{E}$ values of the soma and processes before exposure to $\mathrm{A} \beta_{1-42}$ oligomers are in Fig. 2b and c, respectively. For example, neurons which were pretreated with nocodazole and then exposed to $A \beta_{1-42}$ aggregates were significantly stiffer than the
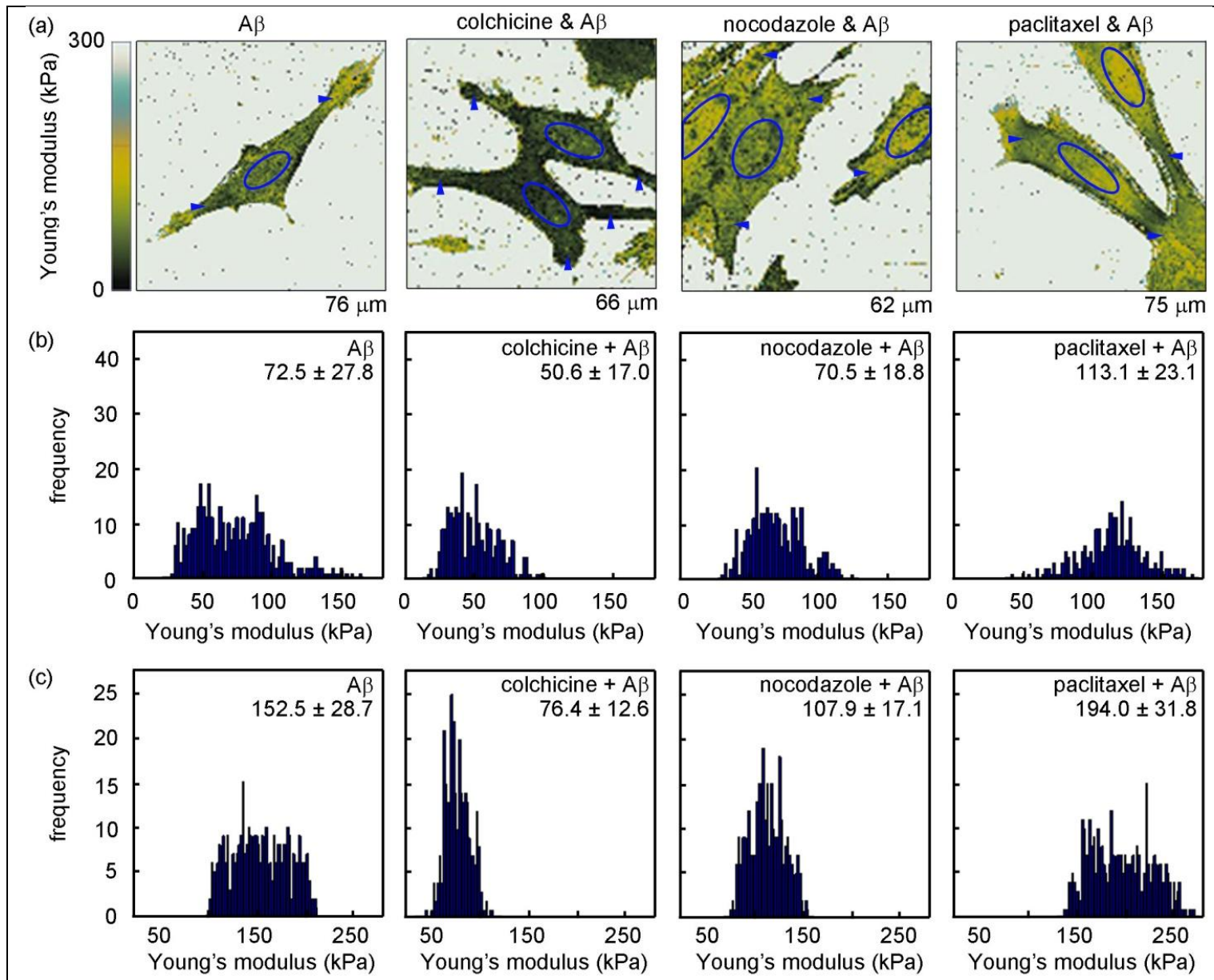

Figure 6. Measuring the Young's modulus of neurons treated with a MT modifier and $\mathrm{A} \beta_{1-42}$. (a) Maps of Young's modulus obtained by force volume imaging. Histograms of the average value of $\mathrm{E}$ were constructed by extracting values from the (b) soma or (c) processes of neurons treated with: the vehicle or MT-altering drug (colchicine, nocodazole, paclitaxel) for 4 hours followed by a 30 minute exposure to oligomeric $A \beta_{1 \text { - }}$ 42. Measurements represent the mean $\mathrm{E} \pm$ standard deviation. 
neurons receiving only a nocodazole treatment. Interestingly, stiffening occurred regardless of the state of the MT network at the time of exposure. This effect is consistent with the findings of a similar AFM-based compression study on neurons, where an increase in cell stiffness was observed after exposure to $5 \mu \mathrm{M}$ oligomeric $\mathrm{A} \beta_{1-42}$ solutions for 30 minutes [100].

\subsubsection{Microtubule disruption reduces the ability of neurons to cope with an $A \beta_{1-42}$ exposure}

After the appropriate controls were performed to examine physical, mechanical, and aggregate-membrane binding changes resulting from neuronal MT modification, it was then possible to examine the influence of MT modification on the cellular response to $\mathrm{A} \beta_{1-42}$ aggregate exposure. Plasma membrane (PM) degradation and MTT reduction assays were used for this investigation, as these assays are effective tools for assessing A $\beta$-induced toxicity [83, 101-105]. Neurons were pretreated with an MT-disruptor (colchicine or nocodazole) or the vehicle (DMSO), at concentrations ranging from 0.1 to $10 \mu \mathrm{M}$ for 4 hours, and then $5 \mu \mathrm{M} \mathrm{A} \beta_{1-42}$ was added to the culture medium for an additional 24 to 48 hours. The MT modifiers were not removed from the culture medium during the $A \beta_{1-42}$ incubation to prevent MT recovery. As an important note, the amount PM degradation or MTT reduction for each sample is plotted relative to the control, i.e., the total amount of PM degradation or MTT reduction for neurons that received a 28 or 52 hour treatment with the vehicle only (DMSO).

When neurons were exposed to $0.1-10 \mu \mathrm{M}$ colchicine, there was no statistical difference in the PM degradation induced by the various colchicine concentrations at each respective time point (white bars to the left, Fig. 7a). However, when neurons were pretreated with colchicine at each concentration and then exposed to $A \beta_{1-42}$ aggregates for an additional 24 or 48 hours (gray bars in the center), the PM degradation significantly increased by $126-202 \%$ as compared to the neurons that received only a colchicine treatment for 28 or 52 hours. In some cases, neurons with a coexposure to both colchicine and $A \beta_{1-42}$ (gray bars in the center), experienced 30-59\% more PM degradation than samples exposed to $A \beta_{1-42}$ aggregates alone (black bars to the right). This demonstrates that MT disruption by colchicine enhances the toxicity of $A \beta_{1-42}$ aggregates, especially 

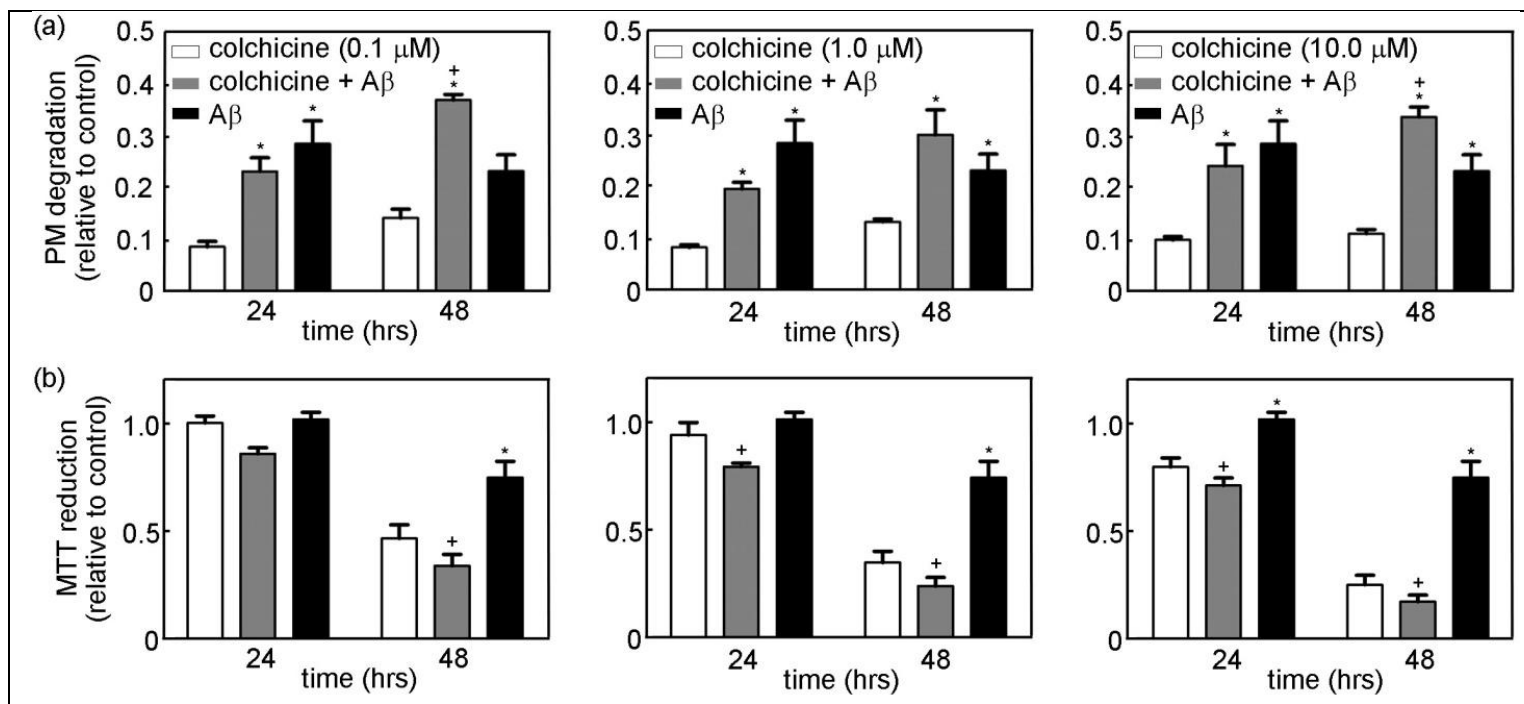

Figure 7. Cellular response to $A \beta_{1-42}$ after colchicine treatment. (a) PM degradation and (b) MTT reduction was evaluated after neurons were pretreated with the vehicle (DMSO), or MT-destabilizing drug (colchicine) followed by a 24 or 48 hour exposure to $5 \mu \mathrm{M} A \beta$. Bars are plotted as relative to the vehicle control (1.0). White bars represent neurons treated with colchicine only, gray bars represent treatment with both colchicine and $A \beta_{1-42}$, and black bars represent treated with $A \beta_{1-42}$ only. The concentration of the drug treatment is shown in the legend of (a). The $(*)$ represents samples found to be statistically different from neurons only treated with colchicine, and the $(+)$ indicates samples that are statistically different from the $\mathrm{A} \beta_{1-42}$ exposed control $(p<0.05)$. Error bars represent standard error of the mean (SEM).

considering that the neurons with disrupted MTs had only half as much aggregatemembrane binding than the neurons without MT disruption.

There was little difference in MTT reduction of the neurons exposed to $0.1 \mu \mathrm{M}$ colchicine, $A \beta_{1-42}$ aggregates, or coexposure to both agents after 24 hours of treatment (Fig. 7b). However, exposure to higher concentrations of colchicine (or longer exposures to $0.1 \mu \mathrm{M}$ colchicine) with or without the presence of $\mathrm{A} \beta_{1-42}$, the MTT response significantly decreased by $22-70 \%$ compared to neurons that were exposed only to $A \beta_{1-42}$ aggregates. This supports the findings from the plasma membrane degradation study; MT disruption by colchicine reduces neuronal viability, and therefore, the neurons are unable to efficiently cope with even low levels of membrane bound $A \beta_{1-42}$ aggregates.

Neurons that received a 28 or 52 hour treatment with nocodazole experienced slight increases in PM degradation as the concentration increased from 0.1-10.0 $\mu \mathrm{M}$, but that amount was not statistically different (white bars to the left, Fig. 8a). When neurons pretreated with nocodazole for four hours were exposed to $A \beta_{1-42}$ aggregates for an 

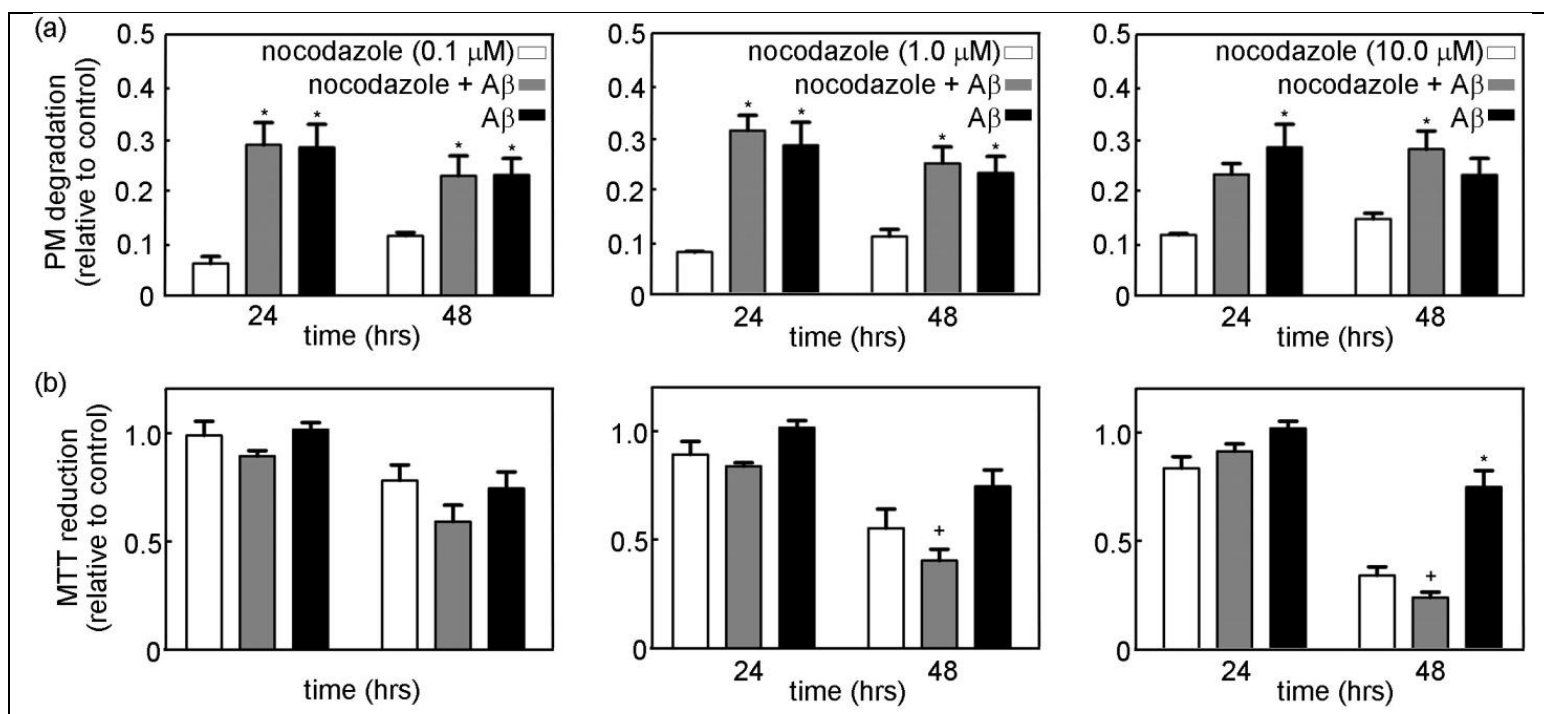

Figure 8. Cellular response to $A \beta_{1-42}$ after nocodazole treatment. (a) PM degradation and (b) MTT reduction was evaluated after neurons were pretreated with the vehicle (DMSO), or MT-destabilizing drug (nocodazole), followed by a 24 or 48 hour exposure to $5 \mu \mathrm{M} \mathrm{A} \beta_{1-42}$. Bars are plotted as relative to the vehicle control (1.0). White bars represent neurons treated with nocodazole only, gray bars represent treatment with both nocodazole and $A \beta_{1-42}$, and black bars represent neurons treated with $A \beta_{1-42}$ only. The concentration of the drug treatment is shown in the legend of (a). The (*) represents samples found to be statistically different from neurons only treated with nocodazole, and the $(+)$ indicates samples that are statistically different from the $A \beta_{1-42}$ exposed control $(p$ $<0.05)$. Error bars represent standard error of the mean (SEM).

additional 24 or 48 hours, PM degradation increased significantly by $99-365 \%$ for cells treated with 0.1 and $1.0 \mu \mathrm{M}$ nocodazole at 24 and 48 hours, as compared to the neurons receiving only a 0.1 or $1.0 \mu \mathrm{M}$ nocodazole treatment. The same trend was not observed when neurons were exposed to $10 \mu \mathrm{M}$ nocodazole treatments; neurons pretreated with 10 $\mu \mathrm{M}$ nocodazole followed by an exposure to $\mathrm{A} \beta_{1-42}$ aggregates for an additional 24 hours did not experience a statistically significant increase in PM degradation as compared to those exposed to $10 \mu \mathrm{M}$ nocodazole alone. This result is likely due to the fact that $10 \mu \mathrm{M}$ nocodazole itself is somewhat more toxic than the lower concentrations of the drug. In some instances, coexposure to the higher concentrations of nocodazole $(1.0$ and $10.0 \mu \mathrm{M})$ and $A \beta_{1-42}$ (gray bar in the center) caused small (7-17\%) increases in PM degradation compared to the neurons that received a vehicle treatment for four hours plus exposure to $\mathrm{A} \beta_{1-42}$ aggregates for an additional 24 or 48 hours (black bar to the right). 
Interestingly, the MTT reduction of neurons exposed to nocodazole, $A \beta_{1-42}$ aggregates, or a combination thereof for 24 hours was essentially analogous (Fig. 8b). After 48 hours of coexposure to $1.0 \mu \mathrm{M}$ nocodazole and $\mathrm{A} \beta_{1-42}$ (gray bar in the center), a $45 \%$ drop in MTT response was observed, as compared to the neurons treated with $A \beta_{1-42}$ only (black bars to the right). Considering that neurons pretreated with nocodazole experienced only half as much aggregate-membrane binding, this indicates that neurons with disrupted MTs were more susceptible to toxic effects of $A \beta_{1-42}$ aggregates. After 48 hours, exposure to $10 \mu \mathrm{M}$ nocodazole alone or a combination of nocodazole and $\mathrm{A} \beta_{1-42}$ aggregates caused $18 \%$ and $54 \%$ reductions in MTT response, respectively, compared to neurons treated only with $A \beta_{1-42}$ indicating that nocodazole itself can cause a large reduction in MTT response at high concentrations.

Overall, the results from the experiments using nocodazole as a MT disruptor showed similar trends to those using colchicine; however, the toxic effects were less severe in the nocodazole treated samples. This could be attributed to the different binding mechanisms of the MT disruptors to tubulin which produce distinct mechanical responses, or a reduction in axonal transport and signaling caused by the breakdown of MTs. In any case, however, the MT disrupted samples treated with $\mathrm{A} \beta_{1-42}$ aggregates experienced just as much, or in some instances, greater toxicity than neurons treated with $\mathrm{A} \beta_{1-42}$ alone. This result suggests that MT disruption interferes with the cellular response mechanisms to $A \beta_{1-42}$-mediated toxicity. This notion is supported by our data that shows neurons pretreated with colchicine and nocodazole experienced half the amount of $A \beta_{1-42}$ aggregate-membrane binding as the neurons that were exposed to the peptide only (Fig. 4b). Therefore, although MT disrupted neurons had lower levels of aggregate-membrane binding, they were unable to efficiently cope with the toxicity caused by $A \beta_{1-42}$ aggregate exposure.

\subsubsection{Microtubule stabilization delays the onset of $A \beta_{1-42}$-induced toxicity}

After observing that MT disruption reduces the ability of neurons to endure $A \beta_{1-42}$ aggregate exposures, we wanted to determine whether MT stabilization could induce a protective effect against $A \beta_{1-42}$ aggregates. Neurons were pretreated with the MT stabilizer, paclitaxel, or the vehicle (DMSO), at concentrations ranging from $0.1-10 \mu \mathrm{M}$ 

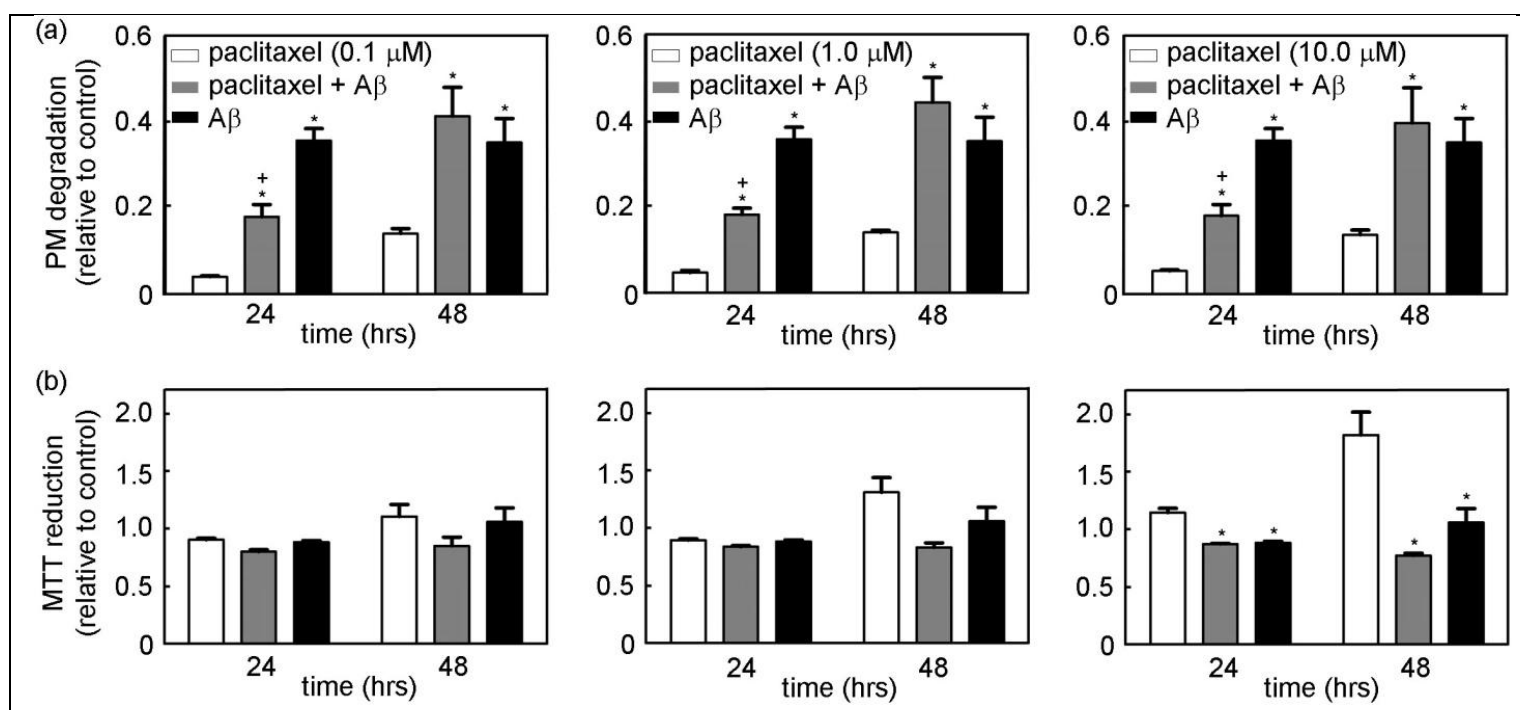

Figure 9. Cellular response to $\mathrm{A} \beta_{1-42}$ after colchicine treatment. (a) PM degradation and (b) MTT reduction was evaluated after neurons were pretreated with the vehicle (DMSO), or MT-stabilizing drug (paclitaxel) followed by a 24 or 48 hour exposure to 5 $\mu \mathrm{M} \mathrm{A} \beta_{1-42}$. Bars are plotted as relative to the vehicle control (1.0). White bars represent neurons treated with paclitaxel only, gray bars represent treatment with both paclitaxel and oligomeric $A \beta_{1-42}$, and black bars represent neurons treated with $A \beta_{1-42}$ only. The concentration of the drug treatment is shown in the legend of (a). The (*) represents samples found to be statistically different from neurons only treated with paclitaxel, and the $(+)$ indicates samples that are statistically different from the $A \beta_{1-42}$ exposed control $(p$ $<0.05)$. Error bars represent standard error of the mean (SEM).

for 4 hours, and then $5 \mu \mathrm{M} \mathrm{A} \beta_{1-42}$ was added to the culture medium for an additional 24 to 48 hours. Neurons receiving a 28 or 52 hour treatment with paclitaxel alone did not experience any statistical difference in PM degradation (white bars to the left, Fig. 9a). However, when PM degradation significantly increased by $187-333 \%$ when neurons were pretreated with paclitaxel and then exposed to $A \beta_{1-42}$ aggregates (gray bars in the center). Interestingly, at 24 hours, the PM degradation caused by coexposure to paclitaxel and $\mathrm{A} \beta_{1-42}$ aggregates was $49 \%$ lower than the PM degradation induced by $\mathrm{A} \beta_{1-42}$ aggregates alone (black bars to the right). This effect was lost after 48 hours of treatment. This indicates that MT-stabilization by paclitaxel may be able to delay the onset of cytotoxicity mediated by $\mathrm{A} \beta_{1-42}$ aggregates.

There was no statistical difference in the MTT reduction of neurons exposed to paclitaxel at 0.1 or $1.0 \mu \mathrm{M}, \mathrm{A} \beta_{1-42}$ aggregates, or a combination thereof (Fig. 9b). However, the MTT response of neurons treated with $10 \mu \mathrm{M}$ paclitaxel was $23-57 \%$ larger than any other population (including the vehicle control, set a 1.0) after 24 and 48 
hours. This trend of increasing MTT response with increasing concentrations of paclitaxel also appeared in two other independent experiments (data not shown). This likely occurred because the MT network is highly associated with mitochondrial shape, movement and function [106-109], and its stabilization could promote mitochondrial activity. For example, hyperphosphorylation of Tau, which is a critical component to MT stabilization, inhibited mitochondrial function in PC-12 cells and mouse brain cortical neurons [110]. Thus, it is not unreasonable to observe an increase in MTT response after MT-stabilization. Nevertheless, there was no significant difference in MTT reduction between neurons coexposed to paclitaxel (at all concentrations) and $A \beta_{1-42}$ aggregates, or neurons treated with the peptide solution only. Combined with results of the plasma membrane degradation assay, this data suggests that MT-stabilization can improve the cellular response mechanisms to cope with the $A \beta_{1-42}$ aggregate exposure.

\subsection{Discussion}

Our studies sought to determine the role of the MT modification on cytotoxicity mediated by $A \beta_{1-42}$ aggregates. This was accomplished by examining the effects of MT disruption and stabilization in GT1-7 neurons exposed to $5 \mu \mathrm{M} A \beta_{1-42}$. Although over $95 \%$ of neurons in each sample treatment group had membrane bound $\mathrm{A} \beta_{1-42}$ aggregates, MT disruption by colchicine and nocodazole significantly reduced the extent of aggregatemembrane binding per cell. The reduction of aggregate-membrane binding likely occurred due to the compromised nature of the neurons that received an MT disrupting treatment. Alterations in neuronal rigidity caused by MT alterations did not appear to affect aggregate-membrane binding; specifically, colchicine reduced neuronal rigidity and nocodazole increased neuronal rigidity compared to neurons with unaltered MTs. This contrasting effect was unexpected, considering that both colchicine and nocodazole cause MT depolymerization at high concentrations [111], but the discrepancy could be due to the differing binding affinities of each drug to tubulin, and/or their mechanisms of action. Literature reports on the tubulin binding site(s) for nocodazole or colchicine are conflicting based on the species which the tubulin was derived from, and experimental designs [78, 112-118]; however, a common finding is that nocodazole-tubulin binding is reversible, whereas colchicine-tubulin binding is essentially irreversible [78, 111, 117- 
120]. Accordingly, the tight binding of colchicine to tubulin likely contributed to the significant softening of the cells, and to the reduced neuronal viability, compared to nocodazole treated neurons. In any case, the reduction in neuronal viability caused by either MT disruptor significantly enhanced the neurotoxicity of $A \beta_{1-42}$ aggregates. This is supported by the observation that neurons with unaltered MTs had double the amount of membrane bound aggregates per cell, but experienced similar or decreased levels of PM degradation compared to the neurons with disrupted MT networks.

On the contrary, MT stabilization by paclitaxel had a protective effect against $\mathrm{A} \beta_{1-42}$ aggregates, at least for a short time. This finding is further supported by another study which demonstrated a protective effect of MT stabilization against $A \beta$-induced cytotoxicity on primary neuronal cultures. There, neurons were treated with $0.1 \mu \mathrm{M}$ paclitaxel for 2 hours before exposure to either $10 \mu \mathrm{M} \mathrm{A} \beta_{1-42}$ or $\mathrm{A} \beta_{25-35}[103,121,122]$. Importantly, the $A \beta$ solutions used in those studies were prepared by incubations at $37{ }^{\circ} \mathrm{C}$ for 24 hours in $10 \mathrm{mM}$ Tris- $\mathrm{HCl}$, a preparation technique that is known to produce fibrillar aggregates $[53,123]$. Furthermore, only peptide solutions with greater than $70 \%$ $\beta$-sheet conformation were utilized in that study, as confirmed by circular dichroism [103, 121, 122]. Taken together, the observations that paclitaxel-mediated MT stabilization was only able to delay toxicity induced by nonfibrillar aggregates of $\mathrm{A} \beta_{1-42}$, but protected against higher concentrations of fibril-induced toxicity for 48 hours supports the literature that smaller aggregates exert a more toxic effect than their fibrillar counterparts [83, 105, 124].

Regardless of the MT state (disrupted vs. stabilized), neuronal stiffness significantly increased after exposure to $A \beta_{1-42}$ aggregates. This increase in stiffness can be related to the previous discussion point that MT stabilization only delayed aggregateinduced toxicity compared to a more sustained protective effect against fibrillar aggregates. Studies have demonstrated that oligomeric $A \beta_{1-42}$ aggregates caused higher stiffness in neurons than fibrillar $A \beta_{1-42}$ [100]; therefore, $A \beta_{1-42}$-induced increases in cell stiffness are directly related to increased toxicity, as $A \beta_{1-42}$ oligomers have been shown to produce a more toxic effect than fibrils $[83,105,124]$. The mechanism of increased neuronal stiffness can be explained by findings from model membranes and neuronal studies. When model membranes were exposed to $A \beta_{1-40}$, the aggregates caused extensive 
membrane disruption and decreased the mechanical stiffness of the bilayer [125]. In neurons, a mechanical stiffening occurred after N2a neuroblastoma cells and HT22 hippocampal neurons were exposed to $5 \mu \mathrm{M}$ oligomeric $\mathrm{A} \beta_{1-42}$ solutions for 30 minutes [100]. The observation of mechanical contrast between model membranes and whole cells after $\mathrm{A} \beta$ exposure is not unjustified, as mechanical measurements on cells account for the entire cell system rather than just the membrane. Thus, while the plasma membrane of a cell may become disrupted and less stiff after A $\beta$ exposure, overall cell stiffening may be observed due to several factors, or a combination thereof. For example, A $\beta$ aggregates may interact with the membrane thereby altering the fluidity or creating pores, which could increase intracellular osmotic pressure by unregulated ion influx [95, 96, 98-100]. Furthermore, studies have demonstrated that in primary hippocampal neurons, $A \beta_{23-35}$ and $A \beta_{1-42}$ (at concentrations greater than $5 \mu \mathrm{M}$ ) exposure stimulated the production of stress fibers through the activation of p38MAPK (mitogen-associated protein kinase) [126], which could also contribute to overall cell stiffening. Taken together, these findings demonstrate that $\mathrm{A} \beta$-membrane interactions may reduce membrane stiffness while increasing the overall mechanical rigidity of neurons through a variety of mechanisms.

Our results demonstrate that MT modifications can have a direct influence on the toxicity of small $A \beta_{1-42}$ aggregates. MT disruption, a consequence of the normal aging process, enhanced the toxicity of $A \beta_{1-42}$ compared to neurons with unaltered or stabilized MTs. This has further pathological implications for the cell, as both synthetic and humanderived aggregates of $A \beta_{1-42}$ stimulated Tau phosphorylation in a variety of cell cultures [37, 127, 128]. Additionally, in vivo studies have demonstrated that injection of $A \beta_{1-42}$ aggregates into the brains of mice and rhesus monkeys caused Tau phosphorylation and the formation of NFTs $[129,130]$. Considering that the physiological role of Tau is to modulate MT assembly and stability, $A \beta_{1-42}$ aggregate-induced phosphorylation disrupts the proper functioning of Tau thereby destabilizing MTs. This essentially creates a selfsustaining feedback mechanism, where age-related MT disruption enhances the toxic ability of $A \beta_{1-42}$ aggregates to trigger the phosphorylation of Tau and further degrade MTs. This mechanism eventually causes synaptic dysfunction, neuronal death, and the breakdown of neuronal networks that leads to the clinical symptoms of AD. Given the 
evidence that MTs play such a critical role in AD pathology, MT stabilization has been explored as a potential treatment strategy [103, 121, 122, 131-133]. Here, we found that treating neurons with the MT stabilizer, paclitaxel, delayed the onset of toxicity induced by nonfibrillar $A \beta_{1-42}$ aggregates, which correlates well with studies demonstrating that MT stabilization has a more prolonged protective effect against $\mathrm{A} \beta_{1-42}$ fibrils. Future efforts will examine how other age-related cytoskeletal modifications, such as disruption of NFs or the actin network, contribute to $A \beta_{1-42}$ aggregate-induced neurotoxicity.

\subsection{References}

1. Prokop, A., R. Beaven, Y. Qu, and N. Sánchez-Soriano, Using fly genetics to dissect the cytoskeletal machinery of neurons during axonal growth and maintenance. Journal of Cell Science, 2013. 126(11): p. 2331-2341.

2. Iqbal, K., T. Zaidi, G. Wen, I. Grundke-Iqbal, P. Merz, S. Shaikh, H. Wisniewski, I. Alafuzoff, and B. Winblad, Defective brain microtubule assembly in Alzheimer's disease. The Lancet, 1986. 328(8504): p. 421-426.

3. Hirokawa, N. and S. Takeda, Gene targeting studies begin to reveal the function of neurofilament proteins (vol 143, pg 1, 1998). Journal of Cell Biology, 1998. 143(4): p. 1143-1143.

4. Riederer, I.M., P. Robert, R. Porchet, J. Eyer, and B.M. Riederer, Selective changes in the neurofilament and microtubule cytoskeleton of NF-H/LacZ mice. Journal of Neuroscience Research, 2003. 71(2): p. 196-207.

5. Twelvetrees, A., A.G. Hendricks, and E.L.F. Holzbaur, SnapShot: Axonal Transport. Cell, 2012. 149(4): p. 950-U251.

6. Raes, M., Involvement of microtubules in modifications associated with cellular aging. Mutation Research, 1991. 256(2-6): p. 149-168.

7. Uchida, A., H. Yorifuji, V.M.Y. Lee, T. Kishimoto, and S. Hisanaga, Neurofilaments of aged rats: The strengthened interneurofilament interaction and the reduced amount of NF-M. Journal of Neuroscience Research, 1999. 58(2): p. 337-348. 
8. Elder, G.A., V.L. Friedrich, A. Margita, and R.A. Lazzarini, Age-related atrophy of motor axons in mice deficient in the mid-sized neurofilament subunit. Journal of Cell Biology, 1999. 146(1): p. 181-192.

9. Gourlay, C.W. and K.R. Ayscough, The actin cytoskeleton in ageing and apoptosis. Fems Yeast Research, 2005. 5(12): p. 1193-1198.

10. Gourlay, C.W., L.N. Carpp, P. Timpson, S.J. Winder, and K.R. Ayscough, A role for the actin cytoskeleton in cell death and aging in yeast. Journal of Cell Biology, 2004. 164(6): p. 803-809.

11. Parhad, I.M., J.N. Scott, L.A. Cellars, J.S. Bains, C.A. Krekoski, and A.W. Clark, Axonal atrophy in aging is associated with a decline in neurofilament gene expression. Journal of Neuroscience Research, 1995. 41(3): p. 355-366.

12. Vickers, J.C., B.M. Riederer, R.A. Marugg, V. Bueescherrer, L. Buee, A. Delacourte, and J.H. Morrison, Alterations in neurofilament protein immunoreactivity in human hippocampal neurons related to normal aging and Alzheimers disease. Neuroscience, 1994. 62(1): p. 1-13.

13. Saha, S. and N.B. Slepecky, Age-related changes in microtubules in the guinea pig organ of Corti - Tubulin isoform shifts with increasing age suggest changes in micromechanical properties of the sensory epithelium. Cell and Tissue Research, 2000. 300(1): p. 29-46.

14. Raes, M., G. Geuens, M. de Brabander, and J. Remacle, Microtubules and microfilaments in ageing hamster embryo fibroblasts in vitro. Experimental Gerontology, 1983. 18(3): p. 241-254.

15. Raes, M. and J. Remacle, Alteration of the microtubule organization in aging WI38 fibroblasts - a comparative study with embryonic hamster lung fibroblasts. Experimental Gerontology, 1987. 22(1): p. 47-58.

16. Bruusgaard, J.C., K. Liest $\varnothing 1$, and K. Gundersen, Distribution of myonuclei and microtubules in live muscle fibers of young, middle-aged, and old mice. Vol. 100. 2006. 2024-2030.

17. Neely, M.D., K.R. Sidell, D.G. Graham, and T.J. Montine, The lipid peroxidation product 4-hydroxynonenal inhibits neurite outgrowth, disrupts neuronal 
microtubules, and modifies cellular tubulin. Journal of Neurochemistry, 1999. 72(6): p. 2323-2333.

18. Cash, A.D., G. Aliev, S.L. Siedlak, A. Nunomura, H. Fujioka, X. Zhu, A.K. Raina, H.V. Vinters, M. Tabaton, A.B. Johnson, M. Paula-Barbosa, J. Avila, P.K. Jones, R.J. Castellani, M.A. Smith, and G. Perry, Microtubule reduction in Alzheimer's disease and aging is independent of $\tau$ filament formation. Am. J. Pathol., 2003. 162: p. 1623-1627.

19. Davies, D.C., N. Horwood, S.L. Isaacs, and D.M.A. Mann, The effect of age and Alzheimer's disease on pyramidal neuron density in the individual fields of the hippocampal formation. Acta Neuropathologica, 1992. 83(5): p. 510-517.

20. Karbowski, M., J.H. Spodnik, M. Teranishi, M. Wozniak, Y. Nishizawa, J. Usukura, and T. Wakabayashi, Opposite effects of microtubule-stabilizing and microtubule-destabilizing drugs on biogenesis of mitochondria in mammalian cells. Journal of Cell Science, 2001. 114(2): p. 281-291.

21. Praprotnik, D., M.A. Smith, P.L. Richey, H.V. Vinters, and G. Perry, Filament heterogeneity within the dystrophic neurites of senile plaques suggests blockage of fast axonal transport in Alzheimer's disease. Acta Neuropathologica, 1996. 91(3): p. 226-235.

22. Terry, R.D. and R. Katzman, Life span and synapses: will there be a primary senile dementia? Neurobiology of Aging, 2001. 22(3): p. 347-348.

23. Mattson, M.P. and T. Magnus, Aging and neuronal vulnerability. Nat. Rev. Neurosci., 2006. 7: p. 278-294.

24. Selkoe, D.J., Alzheimer's Disease. Cold Spring Harbor Perspectives in Biology, 2011. 3(7).

25. Zempel, H., E. Thies, E. Mandelkow, and E.M. Mandelkow, A $\beta$ oligomers cause localized Ca2+ elevation, missorting of endogenous Tau into dendrites, Tau phosphorylation, and destruction of microtubules and spines. Journal of Neuroscience, 2010. 30(36): p. 11938-11950.

26. Burdick, D., B. Soreghan, M. Kwon, J. Kosmoski, M. Knauer, A. Henschen, J. Yates, C. Cotman, and C. Glabe, Assembly and aggregation properties of 
synthetic Alzheimer's amyloid $\beta$ peptide analogs. Journal of Biological Chemistry, 1992. 267(1): p. 546-554.

27. Jarrett, J.T., E.P. Berger, and P.T. Lansbury, The carboxy terminus of the $\beta$ amyloid protein is critical for the seeding of amyloid formation - implications for the pathogenesis of Alzheimer's disease. Biochemistry, 1993. 32(18): p. 46934697.

28. Iwatsubo, T., A. Odaka, N. Suzuki, H. Mizusawa, N. Nukina, and Y. Ihara, Visualization of amyloid $\beta$ 42(43) and amyloid $\beta 40$ in senile plaques with endspecific amyloid $\beta$ monoclonals - Evidence that an initially deposited species is amyloid $\beta$ 42(43). Neuron, 1994. 13(1): p. 45-53.

29. Miller, D.L., I.A. Papayannopoulos, J. Styles, S.A. Bobin, Y.Y. Lin, K. Biemann, and K. Iqbal, Peptide compositions of the cerebrovascular and senile plaque core amyloid deposits of Alzheimer's disease. Archives of Biochemistry and Biophysics, 1993. 301(1): p. 41-52.

30. Murray, M.M., S.L. Bernstein, V. Nyugen, M.M. Condron, D.B. Teplow, and M.T. Bowers, Amyloid $\beta$ protein: A $\beta 40$ inhibits $A \beta 42$ oligomerization. Journal of the American Chemical Society, 2009. 131(18).

31. Yan, Y. and C. Wang, $A \beta 40$ protects non-toxic $A \beta 42$ monomer from aggregation. Journal of Molecular Biology, 2007. 369(4): p. 909-916.

32. Pauwels, K., T.L. Williams, K.L. Morris, W. Jonckheere, A. Vandersteen, G. Kelly, J. Schymkowitz, F. Rousseau, A. Pastore, L.C. Serpell, and K. Broersen, Structural basis for increased toxicity of pathological A $\beta 42: A \beta 40$ ratios in Alzheimer disease. Journal of Biological Chemistry, 2012. 287(8): p. 5650-5660.

33. Kuperstein, I., K. Broersen, I. Benilova, J. Rozenski, W. Jonckheere, M. Debulpaep, A. Vandersteen, I. Segers-Nolten, K. Van Der Werf, V. Subramaniam, D. Braeken, G. Callewaert, C. Bartic, R. D'Hooge, I.C. Martins, F. Rousseau, J. Schymkowitz, and B. De Strooper, Neurotoxicity of Alzheimer's disease $A \beta$ peptides is induced by small changes in the $A \beta 42$ to AB40 ratio. Vol. 29. 2010. 3408-3420.

34. Scheuner, D., C. Eckman, M. Jensen, X. Song, M. Citron, N. Suzuki, T.D. Bird, J. Hardy, M. Hutton, W. Kukull, E. Larson, E. LevyLahad, M. Viitanen, E. Peskind, 
P. Poorkaj, G. Schellenberg, R. Tanzi, W. Wasco, L. Lannfelt, D. Selkoe, and S. Younkin, Secreted amyloid $\beta$-protein similar to that in the senile plaques of Alzheimer's disease is increased in vivo by the presenilin 1 and 2 and APP mutations linked to familial Alzheimer's disease. Nature Medicine, 1996. 2(8): p. 864-870.

35. Duff, K., C. Eckman, C. Zehr, X. Yu, C.-M. Prada, J. Perez-tur, M. Hutton, L. Buee, Y. Harigaya, D. Yager, D. Morgan, M.N. Gordon, L. Holcomb, L. Refolo, B. Zenk, J. Hardy, and S. Younkin, Increased amyloid- $\beta 42(43)$ in brains of mice expressing mutant presenilin 1. Nature, 1996. 383(6602): p. 710-713.

36. Klein, A.M., N.W. Kowall, and R.J. Ferrante, Neurotoxicity and oxidative damage of $\beta$-amyloid 1-42 versus $\beta$-amyloid 1-40 in the mouse cerebral cortex, in Oxidative/Energy Metabolism in Neurodegenerative Disorders, J.P. Blass and F.H. McDowell, Editors. 1999. p. 314-320.

37. De Felice, F.G., D. Wu, M.P. Lambert, S.J. Fernandez, P.T. Velasco, P.N. Lacor, E.H. Bigio, J. Jerecic, P.J. Acton, P.J. Shughrue, E. Chen-Dodson, G.G. Kinney, and W.L. Klein, Alzheimer's disease-type neuronal tau hyperphosphorylation induced by A $\beta$ oligomers. Neurobiology of Aging, 2008. 29(9): p. 1334-1347.

38. Jin, M., N. Shepardson, T. Yang, G. Chen, D. Walsh, and D.J. Selkoe, Soluble amyloid $\beta$-protein dimers isolated from Alzheimer cortex directly induce Tau hyperphosphorylation and neuritic degeneration. Proceedings of the National Academy of Sciences, 2011. 108(14): p. 5819-5824.

39. Takashima, A., K. Noguchi, K. Sato, T. Hoshino, and K. Imahori, Tau protein kinase is essential for amyloid $\beta$ protein induced neurotoxicity. Proceedings of the National Academy of Sciences of the United States of America, 1993. 90(16): p. 7789-7793.

40. Lorenzo, A. and B.A. Yankner, $\beta$-amyloid neurotoxicity requires fibril formation and is inhibited by congo red. Proceedings of the National Academy of Sciences of the United States of America, 1994. 91(25): p. 12243-12247.

41. Busciglio, J., A. Lorenzo, J. Yeh, and B.A. Yankner, $\beta$-amyloid fibrils induce tau phosphorylation and loss of microtubule binding. Neuron, 1995. 14(4): p. 879888. 
42. Ferreira, A., Q. Lu, L. Orecchio, and K.S. Kosik, Selective phosphorylation of adult tau isoforms in mature hippocampal neurons exposed to fibrillar $A \beta$. Molecular and Cellular Neuroscience, 1997. 9(3): p. 220-234.

43. Shea, T.B., A.N. Dergay, and F.J. Ekinci, $\beta$-amyloid induced hyperphosphorylation of tau in human neuroblastoma cells involves map kinase. Neuroscience Research Communications, 1998. 22(1): p. 45-49.

44. Ekinci, F.J., K.U. Malik, and T.B. Shea, Activation of the L voltage-sensitive calcium channel by mitogen-activated protein (MAP) kinase following exposure of neuronal cells to $\beta$-amyloid - Map kinase mediates $\beta$-amyloid-induced neurodegeneration. Journal of Biological Chemistry, 1999. 274(42): p. 3032230327.

45. Cutler, R.G., J. Kelly, K. Storie, W.A. Pedersen, A. Tammara, K. Hatanpaa, J.C. Troncoso, and M.P. Mattson, Involvement of oxidative stress-induced abnormalities in ceramide and cholesterol metabolism in brain aging and Alzheimer's disease. Proceedings of the National Academy of Sciences of the United States of America, 2004. 101(7): p. 2070-2075.

46. Nixon, R.A., The calpains in aging and aging-related diseases. Ageing Research Reviews, 2003. 2(4): p. 407-418.

47. Stokin, G.B., C. Lillo, T.L. Falzone, R.G. Brusch, E. Rockenstein, S.L. Mount, R. Raman, P. Davies, E. Masliah, D.S. Williams, and L.S.B. Goldstein, Axonopathy and transport deficits early in the pathogenesis of Alzheimer's disease. Science, 2005. 307(5713): p. 1282-1288.

48. Mellon, P.L., J.J. Windle, P.C. Goldsmith, C.A. Padula, J.L. Roberts, and R.I. Weiner, Immortalization of hypothalamic GNRH neurons by genetically targeted tumorigenesis. Neuron, 1990. 5(1): p. 1-10.

49. Liposits, Z., I. Merchenthaler, W.C. Wetsel, J.J. Reid, P.L. Mellon, R.I. Weiner, and A. Negrovilar, Morphological characterization of immortalized hypothalamic neurons synthesizing luteinizing hormone-releasing hormone. Endocrinology, 1991. 129(3): p. 1575-1583. 
50. Wetsel, W.C., P.L. Mellon, R.I. Weiner, and A. Negrovilar, Metabolism of proluteinizing hormone releasing hormone in immortalized hypothalamic neurons. Endocrinology, 1991. 129(3): p. 1584-1595.

51. Wetsel, W.C., M.M. Valenca, I. Merchenthaler, Z. Liposits, F.J. Lopez, R.I. Weiner, P.L. Mellon, and A. Negrovilar, Intrinsic pulsatile secretory activity of immortalized luteinizing hormone releasing hormone secreting neurons. Proceedings of the National Academy of Sciences of the United States of America, 1992. 89(9): p. 4149-4153.

52. Whyte, D.B., M.A. Lawson, D.D. Belsham, S.A. Eraly, C.T. Bond, J.P. Adelman, and P.L. Mellon, A neuron specific enhancer targets expression of the gonadotropin releasing hormone gene to hypothalamic neurosecretory neurons. Molecular Endocrinology, 1995. 9(4): p. 467-477.

53. Stine, W.B., K.N. Dahlgren, G.A. Krafft, and M.J. LaDu, In vitro characterization of conditions for amyloid- $\beta$ peptide oligomerization and fibrillogenesis. Journal of Biological Chemistry, 2003. 278(13): p. 11612-11622.

54. Rheinlaender, J., N.A. Geisse, R. Proksch, and T.E. Schäffer, Comparison of scanning ion conductance microscopy with atomic force microscopy for cell imaging. Langmuir, 2010. 27(2): p. 697-704.

55. Hutter, J.L. and J. Bechhoefer, Calibration of atomic-force microscope tips. Review of Scientific Instruments, 1993. 64(7): p. 1868.

56. Burke, K.A., J. Godbey, and J. Legleiter, Assessing mutant huntingtin fragment and polyglutamine aggregation by atomic force microscopy. Methods, 2011. 53(3): p. 275-284.

57. Simakova, O. and N.J. Arispe, The cell-selective neurotoxicity of the Alzheimer's amyloid $\beta$ peptide is determined by surface phosphatidylserine and cytosolic ATP levels. Membrane binding is required for amyloid $\beta$ toxicity. J. Neurosci., 2007. 27(50): p. 13719-29.

58. Smiakova, O. and N.J. Arispe, Fluorescent Analysis of the Cell-Selective Alzheimer's Disease Amyloid $\beta$ Peptide Surface Membrane Binding: Influence of Membrane Components. Int. J. Alzheimer's Dis., 2011: p. 13. 
59. Alenghat, F.J., S.M. Nauli, R. Kolb, J. Zhou, and D.E. Ingber, Global cytoskeletal control of mechanotransduction in kidney epithelial cells. Experimental Cell Research, 2004. 301(1): p. 23-30.

60. Rao, S., G.A. Orr, A.G. Chaudhary, D.G.I. Kingston, and S.B. Horwitz, Characterization of the taxol binding site on the microtubule: 2-(m-azidobenzoyl) taxol photolabels a peptide (amino acids 217-231) of $\beta$-tubulin. Journal of Biological Chemistry, 1995. 270(35): p. 20235-20238.

61. Dennerll, T.J., H.C. Joshi, V.L. Steel, R.E. Buxbaum, and S.R. Heidemann, Tension and compression in the cytoskeleton of PC-12 neurites II - quantative measurements. Journal of Cell Biology, 1988. 107(2): p. 665-674.

62. Joshi, H.C., D. Chu, R.E. Buxbaum, and S.R. Heidemann, Tension and compression in the cytoskeleton of PC-12 neurites. Journal of Cell Biology, 1985. 101(3): p. 697-705.

63. Marx, K.A., T. Zhou, A. Montrone, D. McIntosh, and S.J. Braunhut, A comparative study of the cytoskeleton binding drugs nocodazole and taxol with a mammalian cell quartz crystal microbalance biosensor: Different dynamic responses and energy dissipation effects. Analytical Biochemistry, 2007. 361(1): p. 77-92.

64. Arborgh, B., P. Bell, U. Brunk, and V.P. Collins, The osmotic effect of glutaraldehyde during fixation. A transmission electron microscopy, scanning electron microscopy and cytochemical study. J Ultrastruct Res, 1976. 56: p. 33950.

65. Dulińska, I., M. Targosz, W. Strojny, M. Lekka, P. Czuba, W. Balwierz, and M. Szymoński, Stiffness of normal and pathological erythrocytes studied by means of atomic force microscopy. Journal of Biochemical and Biophysical Methods, 2006. 66(1-3): p. 1-11.

66. Kuznetsova, T.G., M.N. Starodubtseva, N.I. Yegorenkov, S.A. Chizhik, and R.I. Zhdanov, Atomic force microscopy probing of cell elasticity. Micron, 2007. 38(8): p. $824-833$. 
67. Lulevich, V., T. Zink, H.-Y. Chen, F.-T. Liu, and G.-y. Liu, Cell mechanics using atomic force microscopy-based single-cell compression. Langmuir, 2006. 22(19): p. 8151-8155.

68. Zachee, P., J. Snauwaert, P. Vandenberghe, L. Hellemans, and M. Boogaerts, Imaging red blood cells with the atomic force microscope. British Journal of Haematology, 1996. 95(3): p. 472-481.

69. Braet, F., C. Rotsch, E. Wisse, and M. Radmacher, Comparison of fixed and living liver endothelial cells by atomic force microscopy. Applied Physics A: Materials Science \& Processing, 1998. 66(7): p. S575.

70. Le Grimellec, C., E. Lesniewska, C. Cachia, J.P. Schreiber, F. de Fornel, and J.P. Goudonnet, Imaging of the membrane surface of MDCK cells by atomic force microscopy. Biophysical Journal, 1994. 67(1): p. 36-41.

71. Butt, H.J., E.K. Wolff, S.A.C. Gould, B. Dixon Northern, C.M. Peterson, and P.K. Hansma, Imaging cells with the atomic force microscope. Journal of Structural Biology, 1990. 105(1-3): p. 54-61.

72. Mathur, A.B., G.A. Truskey, and W. Monty Reichert, Atomic force and total internal reflection fluorescence microscopy for the study of force transmission in endothelial cells. Biophysical Journal, 2000. 78(4): p. 1725-1735.

73. Tsai, M.A., R.E. Waugh, and P.C. Keng, Passive mechanical behavior of human Neutrophils: effects of colchicine and paclitaxel. Biophysical Journal, 1998. 74(6): p. 3282-3291.

74. Rotsch, C. and M. Radmacher, Drug-induced changes of cytoskeletal structure and mechanics in fibroblasts: An atomic force microscopy study. Biophysical Journal, 2000. 78(1): p. 520-535.

75. Qiu, H., Y. Zhu, Z. Sun, J.P. Trzeciakowski, M. Gansner, C. Depre, R.R.G. Resuello, F.F. Natividad, W.C. Hunter, G.M. Genin, E.L. Elson, D.E. Vatner, G.A. Meininger, and S.F. Vatner, Short Communication: Vascular smooth muscle cell stiffness as a mechanism for increased aortic stiffness with aging. Circulation Research, 2010. 107(5): p. 615-619. 
76. Wu, H.W., T. Kuhn, and V.T. Moy, Mechanical properties of L929 cells measured by atomic force microscopy: Effects of anticytoskeletal drugs and membrane crosslinking. Scanning, 1998. 20(5): p. 389-397.

77. Hyo Il Jung Incheol, S., P. Young Mok, K. Ke Won, and H. Kwon-Soo, Colchicine activates actin polymerization by microtubule depolymerization. Mol. Cells, 1997. 7(3): p. 431-437.

78. Garland, D.L., Kinetics and mechanism of colchicine binding to tubulin: evidence for ligand-induced conformational changes. Biochemistry, 1978. 17(20): p. 42664272.

79. Spedden, E. and C. Staii, Neuron biomechanics probed by atomic force microscopy. Int J Mol Sci, 2013. 14: p. 16124-40.

80. Spedden, E., J.D. White, E.N. Naumova, D.L. Kaplan, and C. Staii, Elasticity maps of living neurons measured by combined fluorescence and atomic force microscopy. Biophys. J., 2012. 103: p. 868-877.

81. McLaurin, J. and A. Chakrabartty, Membrane disruption by Alzheimer $\beta$-amyloid peptides mediated through specific binding to either phospholipids or gangliosides: implications for neurotoxicity. Journal of Biological Chemistry, 1996. 271(43): p. 26482-26489.

82. Canale, C., S. Seghezza, S. Vilasi, R. Carrotta, D. Bulone, A. Diaspro, P.L. San Biagio, and S. Dante, Different effects of Alzheimer's peptide A $\beta(1-40)$ oligomers and fibrils on supported lipid membranes. Biophysical Chemistry, 2013. 182(0): p. 23-29.

83. Deshpande, A., E. Mina, C. Glabe, and J. Busciglio, Different conformations of amyloid $\beta$ induce neurotoxicity by distinct mechanisms in human cortical neurons. Journal of Neuroscience, 2006. 26(22): p. 6011-6018.

84. Mastrangelo, I.A., M. Ahmed, T. Sato, W. Liu, C. Wang, P. Hough, and S.O. Smith, High-resolution atomic force microscopy of soluble Aß42 oligomers. Journal of Molecular Biology, 2006. 358(1): p. 106-119.

85. Cizas, P., R. Budvytyte, R. Morkuniene, R. Moldovan, M. Broccio, M. Lösche, G. Niaura, G. Valincius, and V. Borutaite, Size-dependent neurotoxicity of $\beta$-amyloid oligomers. Archives of Biochemistry and Biophysics, 2010. 496(2): p. 84-92. 
86. Blackley, H.K.L., G.H.W. Sanders, M.C. Davies, C.J. Roberts, S.J.B. Tendler, and M.J. Wilkinson, In-situ atomic force microscopy study of $\beta$-amyloid fibrillization. Journal of Molecular Biology, 2000. 298(5): p. 833-840.

87. Harper, J.D., C.M. Lieber, and P.T. Lansbury Jr, Atomic force microscopic imaging of seeded fibril formation and fibril branching by the Alzheimer's disease amyloid- $\beta$ protein. Chemistry \& Biology, 1997. 4(12): p. 951-959.

88. Reinert, K.C., R.L. Dunbar, W. Gao, G. Chen, and T.J. Ebner, Flavoprotein autofluorescence imaging of neuronal activation in the cerebellar cortex in vivo. Vol. 92. 2004. 199-211.

89. Chance, B., L. Mela, and D. Wong, Flavins and flavoproteins, in Proceedings of the 2nd Conference on Flavins and Flavoproteins, Y. Kunio, Editor 1968, Univ. of Tokyo Press: Tokyo.

90. Kowalewski, T. and D.M. Holtzman, In situ atomic force microscopy study of Alzheimer's $\beta$-amyloid peptide on different substrates: New insights into mechanism of $\beta$-sheet formation. Proceedings of the National Academy of Sciences, 1999. 96(7): p. 3688-3693.

91. Burke, K.A., E.A. Yates, and J. Legleiter, Biophysical insights into how surfaces, including lipid membranes, modulate protein aggregation related to neurodegeneration. Frontiers in Neurology, 2013. 4.

92. Yip, C.M., A.A. Darabie, and J. McLaurin, A $\beta$ 42-peptide assembly on lipid Bilayers. Journal of Molecular Biology, 2002. 318(1): p. 97-107.

93. Kayed, R., Y. Sokolov, B. Edmonds, T.M. McIntire, S.C. Milton, J.E. Hall, and C.G. Glabe, Permeabilization of lipid bilayers is a common conformationdependent activity of soluble amyloid oligomers in protein misfolding diseases. Journal of Biological Chemistry, 2004. 279(45): p. 46363-46366.

94. Kremer, J.J., M.M. Pallitto, D.J. Sklansky, and R.M. Murphy, Correlation of $\beta$ amyloid aggregate size and hydrophobicity with decreased bilayer fluidity of model membranes. Biochemistry, 2000. 39(33): p. 10309-10318.

95. Quist, A., I. Doudevski, H. Lin, R. Azimova, D. Ng, B. Frangione, B. Kagan, J. Ghiso, and R. Lal, Amyloid ion channels: A common structural link for protein- 
misfolding disease. Proceedings of the National Academy of Sciences of the United States of America, 2005. 102(30): p. 10427-10432.

96. Demuro, A., E. Mina, R. Kayed, S.C. Milton, I. Parker, and C.G. Glabe, Calcium Dysregulation and Membrane Disruption as a Ubiquitous Neurotoxic Mechanism of Soluble Amyloid Oligomers. Journal of Biological Chemistry, 2005. 280(17): p. 17294-17300.

97. Kakio, A., S.-i. Nishimoto, K. Yanagisawa, Y. Kozutsumi, and K. Matsuzaki, Interactions of amyloid $\beta$-protein with various gangliosides in raft-like membranes: importance of GM1 ganglioside-bound form as an endogenous seed for Alzheimer amyloid. Biochemistry, 2002. 41(23): p. 7385-7390.

98. Demuro, A., I. Parker, and G.E. Stutzmann, Calcium signaling and amyloid toxicity in Alzheimer disease. Journal of Biological Chemistry, 2010. 285(17): p. 12463-12468.

99. Mattson, M.P. and S.L. Chan, Neuronal and glial calcium signaling in Alzheimer's disease. Cell Calcium, 2003. 34(4-5): p. 385-397.

100. Lulevich, V., C.C. Zimmer, H.-s. Hong, L.-w. Jin, and G.-y. Liu, Single-cell mechanics provides a sensitive and quantitative means for probing amyloid- $\beta$ peptide and neuronal cell interactions. Proceedings of the National Academy of Sciences, 2010. 107(31): p. 13872-13877.

101. Walsh, D.M., D.M. Hartley, Y. Kusumoto, Y. Fezoui, M.M. Condron, A. Lomakin, G.B. Benedek, D.J. Selkoe, and D.B. Teplow, Amyloid $\beta$-protein fibrillogenesis - Structure and biological activity of protofibrillar intermediates. Journal of Biological Chemistry, 1999. 274(36): p. 25945-25952.

102. Piccini, A., C. Russo, A. Gliozzi, A. Relini, A. Vitali, R. Borghi, L. Giliberto, A. Armirotti, C. D'Arrigo, A. Bachi, A. Cattaneo, C. Canale, S. Torrassa, T.C. Saido, W. Markesbery, P. Gambetti, and M. Tabaton, $\beta$-amyloid Is different in normal aging and in Alzheimer disease. Journal of Biological Chemistry, 2005. 280(40): p. 34186-34192.

103. Michaelis, M.L., S. Ansar, Y. Chen, E.R. Reiff, K.I. Seyb, R.H. Himes, K.L. Audus, and G.I. Georg, $\beta$-amyloid-induced neurodegeneration and protection by 
structurally diverse microtubule-stabilizing agents. Journal of Pharmacology and Experimental Therapeutics, 2005. 312(2): p. 659-668.

104. LIN, H., R. BHATIA, and R. LAL, Amyloid $\beta$ protein forms ion channels: implications for Alzheimer's disease pathophysiology. The FASEB Journal, 2001. 15(13): p. 2433-2444.

105. Choi, Y.J., S. Chae, J.H. Kim, K.F. Barald, J.Y. Park, and S.H. Lee, Neurotoxic amyloid $\beta$ oligomeric assemblies recreated in microfluidic platform with interstitial level of slow flow. Scientific Reports, 2013. 3.

106. Heggeness, M.H., M. Simon, and S.J. Singer, Association of mitochondria with microtubules in cultured cells. Proceedings of the National Academy of Sciences of the United States of America, 1978. 75(8): p. 3863-3866.

107. Ball, E.H. and S.J. Singer, Mitochondria are associated with microtubules and not with intermediate filaments in cultured fibroblasts. Proceedings of the National Academy of Sciences of the United States of America-Biological Sciences, 1982. 79(1): p. 123-126.

108. Anesti, V. and L. Scorrano, The relationship between mitochondrial shape and function and the cytoskeleton. Biochimica Et Biophysica Acta-Bioenergetics, 2006. 1757(5-6): p. 692-699.

109. Fuchs, F., H. Prokisch, W. Neupert, and B. Westermann, Interaction of mitochondria with microtubules in the filamentous fungus Neurospora crassa. Journal of Cell Science, 2002. 115(9): p. 1931-1937.

110. Shahpasand, K., I. Uemura, T. Saito, T. Asano, K. Hata, K. Shibata, Y. Toyoshima, M. Hasegawa, and S. Hisanaga, Regulation of mitochondrial transport and inter-microtubule spacing by Tau phosphorylation at the sites hyperphosphorylated in Alzheimer's disease. Journal of Neuroscience, 2012. 32(7): p. 2430-2441.

111. De Brabander, M.J., R.M.L. Van de Velre, F.E.M. Aerts, M. Borgers, and P.A.J. Janssen, The effects of methyl [5-(2-thienylcarbonyl)-1H-benzimidazol-2yl]carbamate, ( $R$ 17934; NSC 238159), a new synthetic antitumoral drug interfering with microtubules, on mammalian cells cultured in vitro. Cancer Research, 1976. 36(3): p. 905-916. 
112. Bryan, J., Vistabline and microtubules: Definition of 3-classes of binding-sites in isolated microtubule crystals. Biochemistry, 1972. 11(14): p. 2611-\&.

113. Owellen, R.J., A.H. Owens, and D.W. Donigian, Binding of vincristine, vistabline and colchicine to tubulin. Biochemical and Biophysical Research Communications, 1972. 47(4): p. 685-\&.

114. Xu, K., P.M. Schwarz, and R.F. Luduena, Interaction of nocodazole with tubulin isotypes. Drug Development Research, 2002. 55(2): p. 91-96.

115. Williams, R.F., M.J. Aivaliotis, L.D. Barnes, and A.K. Robinson, High performance liquid-chromatographic application of the Hummel and Dreyer method for the determination of colchicine-tubulin binding parameters. Journal of Chromatography, 1983. 266(AUG): p. 141-150.

116. Ringel, I. and H. Sternlicht, C-13 NMR study of microtubule protein- evidence for a 2nd colchicine site involved in the inhibition of microtubule assembly. Biochemistry, 1984. 23(23): p. 5644-5653.

117. Hoebeke, J., G. Vannijen, and M. Debrabander, Interaction of oncodazole (R17934), new anti-tumoral drug, with rat brain tubulin. Biochemical and Biophysical Research Communications, 1976. 69(2): p. 319-324.

118. Head, J., L.L.Y. Lee, D.J. Field, and J.C. Lee, Equilibrium and rapid kinetic studies on nocodazole-tubulin interaction. Journal of Biological Chemistry, 1985. 260(20): p. 1060-1066.

119. Samson, F., J.A. Donoso, I. Hellerbettinger, D. Watson, and R.H. Himes, Nocodazole action on tubulin assembly, axonal ultrastructure and fast axoplasmic transport. Journal of Pharmacology and Experimental Therapeutics, 1979. 208(3): p. 411-417.

120. Zieve, G.W., D. Turnbull, J.M. Mullins, and J.R. McIntosh, Production of large numbers of mitotic mammalian cells by use of the reversible microtubule inhibitor nocodazole- nocodazole accumulated mitotic cells. Experimental Cell Research, 1980. 126(2): p. 397-405.

121. Michaelis, M.L., N. Ranciat, Y. Chen, M. Bechtel, R. Ragan, M. Hepperle, Y. Liu, and G. Georg, Protection against $\beta$-amyloid toxicity in primary neurons by paclitaxel (Taxol). Journal of Neurochemistry, 1998. 70(4): p. 1623-1627. 
122. Michaelis, M.L., Y.X. Chen, S. Hill, E. Reiff, G. Georg, A. Rice, and K. Audus, Amyloid peptide toxicity and microtubule-stabilizing drugs. Journal of Molecular Neuroscience, 2002. 19(1-2): p. 101-105.

123. Nilsson, M.R., Techniques to study amyloid fibril formation in vitro. Methods, 2004. 34(1): p. 151-160.

124. Dahlgren, K.N., A.M. Manelli, W.B. Stine, L.K. Baker, G.A. Krafft, and M.J. $\mathrm{LaDu}$, Oligomeric and fibrillar species of amyloid- $\beta$ peptides differentially affect neuronal viability. Journal of Biological Chemistry, 2002. 277(35): p. 3204632053.

125. Burke, K.A., E.A. Yates, and J. Legleiter, Amyloid-forming proteins alter the local mechanical properties of lipid membranes. Biochemistry, 2013. 52: p. 808817.

126. Song, C., G. Perides, D. Wang, and Y.F. Liu, $\beta$-Amyloid peptide induces formation of actin stress fibers through p38 mitogen-activated protein kinase. Journal of Neurochemistry, 2002. 83(4): p. 828-836.

127. Ma, Q.-L., F. Yang, E.R. Rosario, O.J. Ubeda, W. Beech, D.J. Gant, P.P. Chen, B. Hudspeth, C. Chen, Y. Zhao, H.V. Vinters, S.A. Frautschy, and G.M. Cole, $\beta$ amyloid oligomers induce phosphorylation of Tau and inactivation of insulin receptor substrate via c-Jun $N$-terminal kinase signaling: suppression by omega-3 fatty acids and curcumin. The Journal of Neuroscience, 2009. 29(28): p. 90789089.

128. Esposito, G., D. De Filippis, R. Carnuccio, A. Izzo, and T. Iuvone, The marijuana component cannabidiol inhibits $\beta$-amyloid-induced tau protein hyperphosphorylation through Wnt/ $\beta$-catenin pathway rescue in PC12 cells. Journal of Molecular Medicine, 2006. 84(3): p. 253-258.

129. Götz, J., F. Chen, J. van Dorpe, and R.M. Nitsch, Formation of neurofibrillary tangles in P301L Tau transgenic mice induced by Aß42 fibrils. Science, 2001. 293(5534): p. 1491-1495.

130. Geula, C., C.K. Wu, D. Saroff, A. Lorenzo, M.L. Yuan, and B.A. Yankner, Aging renders the brain vulnerable to amyloid $\beta$-protein neurotoxicity. Nature Medicine, 1998. 4(7): p. 827-831. 
131. Lee, V.M.Y., R. Daughenbaugh, and J.Q. Trojanowski, Microtubule stabilizing drugs for the treatment of Alzheimer's disease. Neurobiology of Aging, 1994. 15: p. S87-S89.

132. Ballatore, C., K.R. Brunden, D.M. Huryn, J.Q. Trojanowski, V.M.Y. Lee, and A.B. Smith, Microtubule Stabilizing Agents as Potential Treatment for Alzheimer's Disease and Related Neurodegenerative Tauopathies. Journal of Medicinal Chemistry, 2012. 55(21): p. 8979-8996.

133. Zhang, B., J. Carroll, J.Q. Trojanowski, Y.M. Yao, M. Iba, J.S. Potuzak, A.M.L. Hogan, S.X. Xie, C. Ballatore, A.B. Smith, V.M.Y. Lee, and K.R. Brunden, The microtubule-stabilizing agent, epothilone D, reduces axonal dysfunction, neurotoxicity, cognitive deficits, and Alzheimer-like pathology in an interventional study with aged Tau transgenic mice. Journal of Neuroscience, 2012. 32(11): p. 3601-3611. 
Investigating the role of mechanical properties in biological systems

\author{
Nicole Shamitko-Klingensmith
}

Dissertation submitted to the Eberly College of Arts and Sciences at West Virginia University in partial fulfillment of the requirements for the degree of

Doctor of Philosophy in Chemistry

C. Eugene Bennett Department of Chemistry

APPROVAL OF THE EXAMINING COMMITTEE
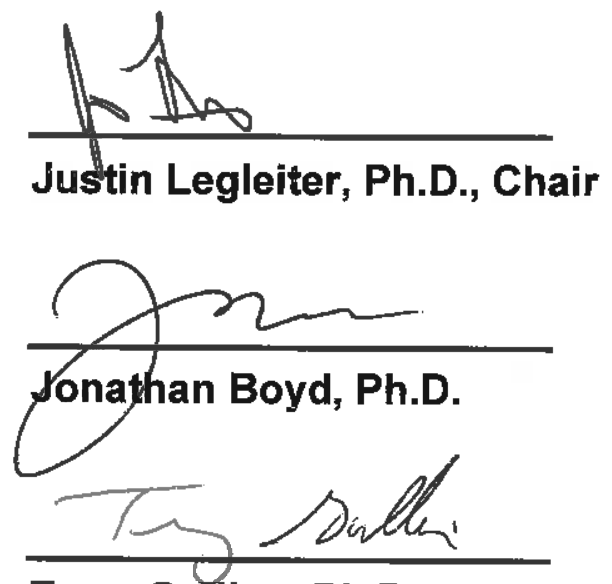

Terry Gullion, Ph.D.

michelle Bichands batet

Michelle Richards-Babb, Ph.D.

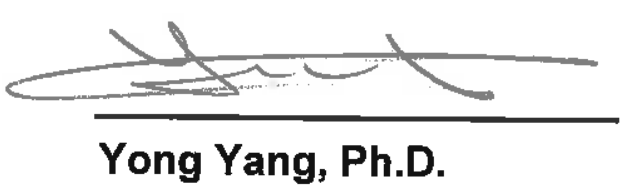

